\title{
Multi-particle interactions in hadronic transport approaches
}

\author{
DisSERTATION \\ zur Erlangung des Doktorgrades \\ der Naturwissenschaften \\ Doctor philosophiae naturalis \\ vorgelegt beim Fachbereich Physik der \\ Johann Wolfgang Goethe-Universität \\ in Frankfurt am Main \\ von \\ Jan Staudenmaier \\ aus Frankfurt am Main
}

Frankfurt 2021

(D 30) 
vom Fachbereich Physik (13) der

Johann Wolfgang Goethe-Universität als Dissertation angenommen.

Dekan: Prof. Dr. Harald Appelshäuser

Gutachter: Prof. Dr. Hannah Elfner und Priv. Doz. Dr. Hendrik van Hees Datum der Disputation: 19. Oktober 2021 


\title{
Zusammenfassung
}

\author{
Diese Dissertation basiert auf folgenden Publikationen:
}

1. Dilepton production and resonance properties within a new hadronic transport approach in the context of the GSI-HADES experimental data. J. Staudenmaier, J. Weil, V. Steinberg, S. Endres \& H. Petersen, Phys. Rev. C 98, 054908 (2018) [1].

2. Strangeness production via resonances in heavy-ion collisions at energies available at the GSI Schwerionensynchrotron. V. Steinberg, J. Staudenmaier, D. Oliinychenko, F. Li, Ö. Erkiner, H. Elfner, Phys. Rev. C 99, 064908 (2019) [2].

3. Particle production in $\mathrm{AgAg}$ collisions at $\mathrm{E} \mathrm{Kin}=1.58 \mathrm{~A} \mathrm{GeV}$ within a hadronic transport approach. J. Staudenmaier, N. Kübler \& H. Elfner, Phys. Rev. C 103, 044904 (2021) [3].

4. 'Smashing more than two': Deuteron production in relativistic heavy ion collisions via stochastic multi-particle reactions. J. Staudenmaier, D. Oliinychenko, J. M. Torres-Rincon \& H. Elfner, arXiv:2106.14287 [hep-ph] (2021) [4].

5. The role of proton-antiproton regeneration in the late stages of heavy-ion collisions. O. Garcia-Montero, J. Staudenmaier, A. Schäfer, J. M. Torres-Rincon \& H. Elfner, arXiv:2107.08812 [hep-ph] (2021) [5].

Die Frage nach den Eigenschaften der starken Wechselwirkung und der (Atom-)Kernmaterie, die durch sie zusammengehalten wird, ist eine der grundlegenden Forschungsfragen, die von einigen der weltweit größten Experimente untersucht wird. In diesen TeilchenbeschleunigerExperimenten werden schwere Atomkerne (Schwerionen) mit fast Lichtgeschwindigkeit zur Kollision gebracht, um ein extrem heißes und dichtes Medium zu erzeugen, an welchem man die Eigenschaften und Phänomene einer der vier Grundkräfte, der starken Wechselwirkung, untersuchen kann. Das Medium ist so heiß und dicht, dass der gleiche Zustand wie kurz nach dem Urknall erreicht wird. Hierbei schmelzen die Nukleonen, die Atomkern-Bestandteile, und ein neuer Phasenzustand der Materie, ein Plasma aus Quarks und Gluonen, entsteht. Experimentell nachweislich wird ein Quark-Gluon-Plasma in den Teilchenbeschleunigern mit den höchsten Kollisionsenergien erzeugt: dem Relativistic Heavy Ion Collider (RHIC) am Brookhaven National Laboratory und im Large Hadron Collider (LHC) am CERN. Quarks und Gluonen (auch gemeinsam als Partonen bezeichnet) sind hierbei die elementaren Freiheitsgrade der starken Wechselwirkung. Gluonen sind die Kraft-Austauschteilchen und Quarks die fundamentalen Bestandteile der Hadronen, wie aus Quarks zusammengesetzte Teilchen genannt werden. Auch Nukleonen sind Hadronen. Die starke Wechselwirkung verdankt ihren Namen der Stärke ihrer Kopplung, die durch die Kopplungskonstante charakterisiert ist. Bei alltäglichen, niedrigen Energien ist die Kopplungskonstante der starken Wechselwirkung groß. Die starke Kopplung führt zum Einschluss (Confinement) von Quarks und Gluonen in Hadronen. Freie Quarks und Gluonen kommen nicht natürlich vor. Bei hohen Energien und damit verbundenen kurzen Längenskalen sinkt die Kopplungsstärke allerdings, da die Kopplungskonstante energieabhängig ist. Man spricht von asymptotischer Freiheit, weil die elementaren Teilchen sich bei diesen Energieskalen frei verhalten. Dieses Phänomen der starken Wechselwirkung diente, 
als eine der ursprünglichen Motivationen Schwerionen bei hoher Energie zur Kollision zu bringen, um ein Plasma aus freien Quarks und Gluonen (Quark-Gluon-Plasma) zu erzeugen. Der Verlauf einer hochenergetischen Schwerionenkollision hat folglich verschiedene Phasen mit verschiedenen Freiheitsgraden. Die aus Hadronen bestehenden Atomkerne kollidieren und erzeugen eine extrem heiße und dichte Phase, in der ein Quark-Gluon-Plasma entsteht. Kühlt das System durch Expansion ab, formt sich wieder ein Gas aus Hadronen. Bei niedrigeren Strahlenergien verbleiben die Freiheitsgrade hadronisch und es entsteht kein Quark-Gluon-Plasma.

Diese Arbeit beschäftigt sich mit der theoretischen Beschreibung von diesen hadronischen Phasen in Schwerionenkollisionen. Die grundlegende Theorie der starken Wechselwirkung, die Quantenchromodynamik, ist nur begrenzt und mit sehr großem Aufwand lösbar. Insbesondere, erlaubt sie keine Beschreibung von dynamischen Systemen, wie Schwerionenkollisionen. Für diese Beschreibung werden deswegen effektive, kinematische Ansätze gewählt. Einer dieser Ansätze sind Transportmodelle, die eine dynamische, mikroskopische Evolution von Hadronen beschreiben können. Dieser Ansatz ist vor allem relevant für die Abschnitte einer Schwerionenkollision, in der kein Gleichgewicht für das beschriebene Medium angenommen werden kann. Ein Zustand, in dem sich zum Beispiel die späten Abschnitte von Kollisionen mit hohen Strahlenergien befinden.

In dieser Arbeit wird ein solches hadronisches Transportmodell (SMASH) verwendet, um zu untersuchen, wie sich das hadronische Medium in Schwerionenkollisionen verhält. Im Speziellen wird sich der Frage gewidmet, was die relevanten mikroskopischen Reaktionen und die Eigenschaften der beschriebenen Freiheitsgrade sind. Dafür wird zuerst die bestehende hadronische Evolution mit binären Kollisionen durch die Emission von Dileptonen und Seltsamkeitsproduktion untersucht. Anschließend wird die Evolution um Mehrteilchenreaktionen erweitert und deren Effekt auf gemessene Observablen studiert. Das Studium von Mehrteilchenreaktionen ist Hauptthema dieser Arbeit. Die verschiedenen Studien dieser Dissertation und deren Ergebnisse sind im Folgenden erklärt und zusammengefasst.

Die Produktion von korrelierten Leptonpaaren (Dileptonen) und von Teilchen, die strange Quarks enthalten (Seltsamkeit), ermöglicht zu überprüfen, ob der verwendete Transportansatz experimentelle Daten bei niedrigen Strahlenergien reproduzieren kann. Beide Proben sind speziell auf das Medium sensitiv, weil Dileptonen das hadronische Medium ungestört verlassen und die Seltsamkeit erst im Medium produziert wird (andere Quarks sind schon Bestandteile der initialen Nukleonen). Deswegen wird anhand dieser Vergleiche validiert, ob Transportansätze, im Speziellen der verwendete (SMASH), in der Lage sind, das hadronische Medium über die vollständige Dauer einer Schwerionenkollision bei niedrigen Energien zu beschreiben. Die Untersuchung der Seltsamkeit fokussiert sich auf die Produktion von $\phi$ und $\Xi$ Hadronen. Es ist es möglich die bisherigen Messungen für die Produktion der beiden doppelt seltsamen Teilchen mit Hilfe eines neuen Produktionsmechanismus, basierend auf dem Zerfall von schweren Nukleon-Resonanzen, zu erklären. Auch die Dileptonproduktion ist für kleine Systeme kompatibel mit den experimentellen Daten. In größeren Systemen ermöglicht sie das Studium von zusätzlichen In-Medium-Effekten. Im invarianten Massenspektrum der Dileptonen lassen sich Änderungen der Vektormeson-Spektralfunktion beobachten. SMASH verwendet konstante Vakuum-Eigenschaften für die Resonanzen, einschließlich der Vektormesonen $(\rho, \omega, \phi)$. Schon bei den betrachteten niedrigen Strahlenergien ist eine Modifikation des invarianten Massenspektrums der Dielektronen, die nicht mit Vakuum-Eigenschaften vereinbar ist, für große Kollisionssysteme zu beobachten. Eine im Medium veränderliche Spektralfunktion lässt sich in einem coarse-graining Modell beschreiben. Da Transport und coarse-graining 
Modell auf der gleichen Evolution basieren, lässt sich die Modifikation im invarianten Massenspektrum dadurch im Detail quantifizieren und auf eine Modifikation der Spektralfunktion der Vektormesonen, wie sie bei der Wiederherstellung der chiralen Symmetrie vorausgesagt wird, zurückführen. Die Verwendung von Vakuum-Eigenschaften in einer hadronischen Transport-Kaskadenrechnung ermöglicht folglich die Isolierung und Quantifizierung von zusätzlichen In-Medium-Effekten.

Die Grundlage von Mehrteilchenreaktionen in Transportmodellen ist ein stochastisches Kollisionskriterium. Dieser Ansatz, auch als stochastische Raten bezeichnet, erlaubt, im Gegensatz zu einem geometrischen Kollisionskriterium auch Mehrteilchenreaktionen zu beschreiben. Das verwendete Transportmodell wird in dieser Arbeit um ein stochastisches Kollisionskriterium und verschiedene Mehrteilchenreaktionen erweitert. Die theoretische Herleitung für die Beschreibung von Mehrteilchenreaktionen mit Hilfe von stochastischen Raten ist anschaulich und ausführlich dargestellt. Ausgehend vom Kollisionsterm der Boltzmann-Gleichung werden Kollisionswahrscheinlichkeiten für 2-, 3- und 5-Teilchenkollisionen hergeleitet und verallgemeinert. Diese Wahrscheinlichkeiten werden unter der Annahme eines konstanten Matrixelementes in Abhängigkeit der Zerfallsbreite oder des Wirkungsquerschnitts des umgekehrten Prozesses angegeben. Die hergeleiteten Formeln dienen als Basis für die Realisierung von Mehrteilchenreaktionen im Transportansatz SMASH. Die folgenden Mehrteilchenreaktionen sind einbezogen: die Rückreaktion von mesonischen Dalitzzerfällen (3-nach-1 Reaktionen), Deuteron-Katalyse (3-nach-2 Reaktionen) und die Rückreaktion von Nukleon-Annihilation (5-nach-2 Reaktionen). Letzteres ist die erste Realisierung einer 5-Körper Reaktion in einem Transportmodell.

Zur Überprüfung der Herleitung und der numerischen Umsetzung des neuen Ansatzes für stochastische Raten in SMASH werden die Ergebnisse in verschiedenen Testsystemen validiert, bevor realistische Schwerionenkollisionen betrachtet werden. In Boxrechnungen wird die Kollisionsrate und der Äquilibrierungsprozess mit analytischen oder vorangegangen Ergebnissen verglichen. Die Gleichgewichtsrechnungen für die stochastischen Raten stimmen in allen Vergleichen mit den analytischen Erwartungen überein. Es ist zu beobachten, insbesondere auch für Mehrteilchenreaktionen, dass im Gleichgewicht beide Reaktionsrichtungen gleich häufig sind (detailed balance Prinzip). Die Etablierung des neuen Ansatzes wird abgeschlossen mit einer Untersuchung der Stabilität der Ergebnisse unter Variation der Rechenparameter. In den Gleichgewichtsrechnungen ist außerdem festzustellen, dass der Äquilibrierungsprozess für Mehrteilchenreaktionen schneller ist. Im Vergleich zu einer Kettenreaktion mit zwischenzeitlichen Resonanzzuständen, die in der gleichen Gesamtreaktion resultiert, ist ein Gleichgewicht mit direkten Mehrteilchenreaktionen schneller hergestellt. Da bisherige Ergebnisse auf Grund der Limitierung auf ein geometrisches Kriterium mehrstufige Kettenreaktionen als effektive Beschreibung von Mehrteilchenreaktionen verwenden, ist die Frage zu klären, inwiefern dieser Unterschied der Äquilibrierungszeit auch in Schwerionenkollisionen einen Effekt hat.

Die Ergebnisse für Mehrteilchenreaktionen in Schwerionenkollisionen sind nach den drei im hadronischen Transportansatz realisierten Reaktionen gegliedert. Zuerst sind die Rückreaktion von mesonischen Dalitzzerfällen bei niedrigen bis mittleren Strahlenergien untersucht. Diese sind $3 \pi \rightarrow \omega, 3 \pi \rightarrow \phi$ und $\pi \pi \eta \rightarrow \eta^{\prime}$. Die Reaktionen von $\omega, \phi$ und $\eta^{\prime}$ sind eine gute erste Probe für stochastische Raten in einem realistischen Kollisionsszenario. Der $\omega$ Beitrag dominiert die summierten 3-nach-1 Reaktionsrate. $\phi$ und $\eta^{\prime}$ Rückreaktion sind keine signifikanten Beiträge. Für die Gesamtdynamik in einer Schwerionenkollision ist der Einfluss der 3-nach-1 Rückreaktionen vernachlässigbar, da sie relativ selten vorkommen. Es ist allerdings zu beobachten, dass durchschnittlich jedem vierte Dalitzzerfall eine Rückreaktion, die die Resonanz regeneriert, folgt. 
Mit Hilfe der Proton- und Deuteron-Mehrteilchenreaktionen werden Kollisionen mit höheren Strahlenergien untersucht. In der späten Phase dieser Kollisionen ist das Medium hadronisch und nicht mehr im Gleichgewicht. Deswegen wird ihre Evolution mit Transportansätzen berechnet. Kombiniert wird dieser Ansatz mit einer hydrodynamischen Beschreibung für die heiße, dichte und teilweise partonische Phase und einem geeigneten Modell, das die initialen Stöße vor der heißen und dichten Phase beschreibt. Der Fokus der Ergebnisse ist die letzte verdünnte Phase nach der hydrodynamischen Evolution, in der die Hadronen (in Mehrteilchenreaktionen) interagieren.

Der Produktionszeitpunkt von Deuteronen in Schwerionenkollisionen ist eine aktuell diskutierte Forschungsfrage. Gegensätzliche Modellannahmen reproduzieren die Messungen der Deuteronproduktion. Koaleszenzmodelle nehmen an, dass Deuteronen sich am Ende der Kollision aus ihren Bestandteilen (Neutron und Proton) formen, wenn diese sich nah im Phasenraum befinden. Demgegenüber nehmen thermische Modelle eine deutlich frühere Formation kurz nach dem Phasenübergang an. Diese Annahme wirft die Frage auf, wie Deuteronen mit einer Bindungsenergie von wenigen $\mathrm{MeV}$ die Temperaturen kurz nach dem Phasenübergang von hunderten $\mathrm{MeV}$ überstehen, ohne aufzubrechen (dies wird anschaulich mit einem "Schneeball in der Hölle" vergleichen). In dieser Arbeit wird dieses Paradox mit der vollständig dynamischen Evolution eines Transportansatzes untersucht. Die beiden genannten Ansätze vernachlässigen jede Reaktionsdynamik für die Deuteronproduktion. In SMASH werden Deuteronen mikroskopisch in den 3-nach-2 Katalysereaktionen $\pi p n \leftrightarrow \pi d, N p n \leftrightarrow N d$ und $\bar{N} p n \leftrightarrow \bar{N} d$ geformt. Mit Hilfe dieses Ansatzes können die beiden Annahmen von thermischen Modellen und Koaleszenzmodellen untersucht werden. Hierfür werden Gold-Gold Kollisionen bei mittlere Strahlenergie in einem Hybridmodell untersucht. Im ersten Szenario wird angenommen, dass die $d$ schon in der heißen und dichten Phase produziert wurden. Die Deuteronanzahl zum Start der Transportevolution entspricht dabei der im thermischen Modell vorhergesagten Anzahl. Die Deuteronen sind Teil der Anfangsbedingung des Transportmodells. In diesem Fall äquilibrieren sich die Katalysereaktionen sofort. Die Nukleonen und Deuteron befinden sich im Gleichgewicht und die Anzahl der Deuteronen bleibt konstant. Die initialen $d$ werden andauernd zerstört und wieder produziert. Deswegen stimmen die Vorhersagen des thermischen Modells mit den experimentellen Daten überein, auch wenn die Deuteronen aufbrechen (die "Schneebälle in der Hölle schmelzen"). Vernachlässigt man $d$ in der Anfangsbedingung für das Transportmodell, so werden sie erst später geformt, analog zu Koaleszenzmodellen. Interessanterweise führen die Katalysereaktionen zu einem Anstieg der Deuteronanzahl in der späten Kollisionsphase, der vergleichbar mit der konstanten Anzahl der $d$ im vorher beschrieben Szenario ist. Die finale Deuteronanzahl ist folglich unabhängig vom Produktionszeitpunkt, wenn die Deuteronproduktion mit 3-nach-2 Katalysereaktionen beschrieben ist. Das erklärt, warum gegensätzliche Modellannahmen für den Produktionszeitpunkt zur gleichen Deuteronanzahl führen können. Die Ergebnisse mit stochastischen Mehrteilchenreaktionen bestätigen vorangegangene Ergebnisse, die die 3-nach-2 Katalyse mit geometrischen mehrstufigen Reaktionen modellieren. Der in Gleichgewichtsrechnungen beobachtete schnellere Äquilibrierungsprozess für die direkten Mehrteilchenreaktionen führt allerdings zu einem schnelleren und erhöhten Anstieg der Deuteronanzahl (für den Fall ohne $d$ als Teil der Anfangsbedingung). Die Unabhängigkeit der Deuteronanzahl von der Anfangsbedingung des Transportmodells ist mit direkten Mehrteilchenreaktionen im Vergleich zur äquivalenten mehrstufigen Reaktionen damit noch deutlicher zu beobachten.

Die Frage, wie der Effekt von inelastische Streuungen zwischen Hadronen auf die Multiplizität in der späten Kollisionsphase ist, ist auch für Protonen relevant. Anders als 
bei Deuteronen überschätzen thermische Modelle die Protonenanzahl. Ein Mechanismus, der die Protonenanzahl in der späten Kollisionsphase mindert, sind Annihilationsreaktionen zwischen Nukleonen und Antinukleonen. Die wichtigste Annihilationsreaktionen ist $\mathrm{p} \overline{\mathrm{p}} \rightarrow 5 \pi$. Die Übereinstimmung mit experimentellen Daten wird durch die Einbeziehung dieser Reaktion verbessert. Ungeklärt ist allerdings die Frage, inwiefern die Rückreaktion $5 \pi \rightarrow \mathrm{p} \overline{\mathrm{p}}$ die Anzahl an Protonen regeneriert, weil diese 5-Teilchenreaktion in bisherigen theoretischen Rechnungen vernachlässigt wird. In dieser Arbeit wird eine solche 5-Teilchenreaktion zum ersten Mal in einer mikroskopischen Beschreibung der späten Kollisionsphase einbezogen. Zur Untersuchung dieser Phase werden Gold-Gold und $\mathrm{Pb}-\mathrm{Pb}$ Kollisionen bei hohen Energien mit einem Hybridmodell untersucht. In den späten Kollisionsphasen, die mit dem Transportmodell beschrieben werden, lässt sich eine $5 \pi \rightarrow \mathrm{p} \overline{\mathrm{p}}$ Rückreaktion für $20 \%$ aller $\mathrm{p} \overline{\mathrm{p}} \rightarrow 5 \pi$ Reaktionen feststellen. Ein Unterschied zwischen einer direkten Mehrteilchenreaktionen und einer mehrstufigen Modellierung der gleichen Reaktion mit binären Reaktionen wird nicht beobachtet. Bei mittleren Rapiditäten wird die Hälfte der Protonen regeneriert, die durch Annihilation vernichtet wurden. Damit haben sowohl die Annihilationsreaktion als auch ihre Rückreaktion signifikanten Einfluss auf die Multiplizität und müssen für eine vollständige Beschreibung der späten Kollisionsphase beachtet werden.

Insgesamt kann durch die Ergebnisse gezeigt werden, dass die mikroskopische Beschreibung von inelastischer Streuung in den späten Phasen von Schwerionenkollision wichtig ist. Insbesondere die Relevanz von Mehrteilchenreaktionen im Hadronengas, die entscheidenden Einfluss auf finale Multiplizitäten haben, ist verdeutlicht. Der beobachtete Unterschied in der Äquilibrierungszeit zwischen mehrstufigen und direkten Mehrteilchenreaktionen wirkt sich nicht signifikant auf die Schlussfolgerungen der Ergebnisse aus. Der Ansatz, Mehrteilchenreaktionen in mehreren binären Schritten zu modellieren, ist somit validiert. Dieser Ansatz ist jedoch auf Reaktionen, für die geeignete Zwischenzustände bekannt sind, beschränkt. Der direkte Ansatz mit stochastischen Raten ist hingegen flexibel für alle Reaktionen anwendbar und rigoros von der Boltzmann-Gleichung abgeleitet, und deswegen letzten Endes theoretisch zu bevorzugen.

Abschließend lassen sich die Untersuchungen in dieser Dissertation wie folgt zusammenfassen:

- Die hadronische Evolution mit binären Reaktionen in Kern-Kern Kollisionen wird bei niedrigen Strahlenergien mit den Transportansatz SMASH untersucht. Die Dileptonproduktion erlaubt, den Ansatz generell zu verifizieren und Mediummodifikationen der Vektormeson-Resonanzeigenschaften zu quantifizieren. Ein neuer Produktionsmechanismus für doppelt seltsame $\phi$ und $\Xi$ Hadronen basierend auf dem Zerfall von schweren Nukleon-Resonanzen führt zu einer Übereinstimmung mit bisherigen Messungen zur deren Produktion.

- Ein neuer Ansatz für stochastische Raten zur mikroskopischen Beschreibung von Mehrteilchenreaktionen wird etabliert. Die theoretische Herleitung der notwendigen Kollisionswahrscheinlichkeiten ist ausgehend vom Kollisionsterm der BoltzmannGleichung umfassend dargestellt. Die Realisierung der stochastischen Raten im Transportmodell SMASH, dessen Evolution mit Mehrteilchenreaktionen erweitert wird, ist ausführlich verifiziert.

- Die um Mehrteilchenreaktionen erweiterte Evolution wird vor allem in den späten hadronischen Phasen von Schwerionenkollision bei mittleren und hohen Energien untersucht. Die Mehrteilchenreaktionen für Deuteronen $(\pi p n \rightarrow \pi d)$ und Protonen $(5 \pi \rightarrow \mathrm{p} \overline{\mathrm{p}})$ in der späten Nicht-Gleichgewichtsphase der Kollision sind essentiell, um die beobachtete Produktion der beiden Spezies zu erklären. 



\section{Contents}

1 Introduction 1

1.1 Strong-interaction matter under extreme conditions . . . . . . . . . . . . 1

1.2 Theoretical description of heavy-ion collisions . . . . . . . . . . . . . . 4

1.2.1 Quantum Chromodynamics . . . . . . . . . . . . . . . . 4

1.2.2 Thermal Models . . . . . . . . . . . . . . . . . . . 6

1.2 .3 Hydrodynamics . . . . . . . . . . . . . . . . . . . . . 8

1.2.4 Transport approaches . . . . . . . . . . . . . . . 10

1.2.5 Hybrid models . . . . . . . . . . . . . . . . . . . . . 11

1.2 .6 Multi-particle reactions . . . . . . . . . . . . . . . . 13

1.3 Observables of interest . . . . . . . . . . . . . . . . . . 14

1.3.1 Chiral symmetry and dilepton production . . . . . . . . . . 14

1.3.2 Strangeness production . . . . . . . . . . . . . . 17

1.3.3 "Snowballs in hell" - deuterons in heavy-ion reactions . . . . . . . 19

1.3.4 The "proton anomaly" . . . . . . . . . . . . . . . . . 21

1.4 Structure of this thesis . . . . . . . . . . . . . . . . . . 22

2 The hadronic transport approach SMASH 25

2.1 Degrees of freedom . . . . . . . . . . . . . . . 26

2.1 .1 Resonances . . . . . . . . . . . . . . . . 26

2.1 .2 Deuterons . . . . . . . . . . . . . . . . . . . . . . 29

2.1.3 Test particle method . . . . . . . . . . . . . . . . 29

2.2 Effective solution of the Boltzmann equation _ . . . . . . . . . 29

2.2.1 Propagation . . . . . . . . . . . . . . . 30

2.2.2 Geometric collision criterion . . . . . . . . . . . . . . . . . 31

2.2 .3 Interactions . . . . . . . . . . . . . . . . . . . 33

2.3 Initial conditions . . . . . . . . . . . . . . . . . . . . 38

2.4 Dilepton production . . . . . . . . . . . . . . . . . . 40

2.4.1 Decay widths and form factors for dilepton decays . . . . . . . . . 41

2.4 .2 Shining method . . . . . . . . . . . . . . . . . . . . . . . . 42

2.4 .3 Coarse-graining approach . . . . . . . . . . . . 43

3 Results with binary collisions $\quad 47$

3.1 Dilepton emission . . . . . . . . . . . . . . . . . . . . . 49

3.1.1 Elementary cross-sections of dilepton emitting particles . . . . . . 50

3.1.2 Results for dilepton production . . . . . . . . . . . . . . . 52

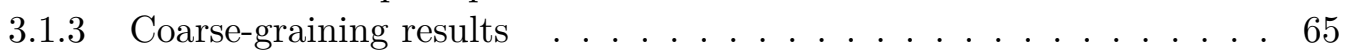

3.1 .4 Summary . . . . . . . . . . . . . . . . . . . 74

3.2 Strangeness production mechanism . . . . . . . . . . . . 76

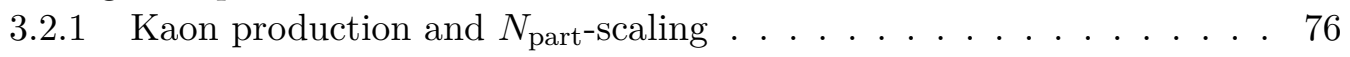



3.2.2 Production of $\phi$ and $\Xi$ from the decay of heavy $N^{*}$ resonances . . . 79

4 Stochastic rates $\quad \mathbf{8 5}$

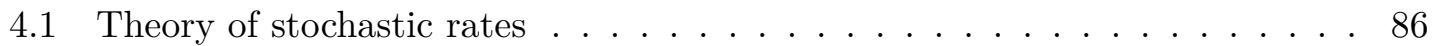

4.1.1 Probability of a collision . . . . . . . . . . . . . 86

4.1 .2 The collision integral . . . . . . . . . . . . . . . . . 87

4.1.3 Probability for 2 -to-2 reactions . . . . . . . . . . . . . . . 91

4.1.4 Probability for 2 -to-m reactions . . . . . . . . . . . . . . 93

4.1.5 Probability for n-to-m reactions . . . . . . . . . . . . . . 93

4.1.6 Test-particle method with stochastic rates . . . . . . . . . . . . . . 94

4.1 .7 Phase space integrals . . . . . . . . . . . . . . . . . . . . . 94

4.1 .8 Decays . . . . . . . . . . . . . . . . . . . . . . . . 97

4.1.9 Resonance formation in 2-to-1 reactions . . . . . . . . . . . . 98

4.1.10 Probability for multi-particle reactions . . . . . . . . . . . . . 100

4.2 Stochastic collision criterion in transport approaches . . . . . . . . . . 106

4.3 Multi-particle reactions in SMASH . . . . . . . . . . . . . . . . 107

4.3.1 Meson 3-to-1 reactions . . . . . . . . . . . . . . . . 108

4.3.2 Deuteron 3 -to-2 reactions . . . . . . . . . . . . . . . . . 108

4.3.3 Proton-antiproton 5 -to-2 reactions . . . . . . . . . . . . . 109

5 Results with multi-particle collisions $\quad \mathbf{1 1 3}$

5.1 Stochastic rates in and out-of equilibrium . . . . . . . . . . . . . . 114

5.1 .1 Elastic scattering rate . . . . . . . . . . . . . . . . 114

5.1.2 Equilibration and detailed balance of the stochastic criterion . . . 116

5.1 .3 Collision rate for an expanding sphere . . . . . . . . . . . . 130

5.1.4 Particle yields and $\left\langle p_{T}\right\rangle$ in nucleus-nucleus collisions . . . . . . . 131

5.1.5 Numerical stability of results with the stochastic criterion . . . . . . 132

5.2 Three-body decay back-reactions in nucleus-nucleus collision . . . . . . . . . 142

5.3 Deuteron production in afterburner calculations . . . . . . . . . . . . . . . 146

5.4 Proton-antiproton regeneration in the late collision stages . . . . . . . . . 154

6 Conclusion and outlook $\quad 161$

A Numerical realization and implementation details of $\begin{array}{lr}\text { the stochastic criterion } & \mathbf{1 8 5}\end{array}$

A.1 Open Software and setup on Github . . . . . . . . . . . . . . 185

A.2 Numerical realization of the stochastic criterion . . . . . . . . . . 186

A.2.1 Grid . . . . . . . . . . . . . . . . . . . 188

A.3 Code design of the ScatterActionMulti class . . . . . . . . . . . . . . 189

A.3.1 Using multi-particle reactions in SMASH . . . . . . . . . . 190

A.4 Performance of multi-particle reactions . . . . . . . . . . . . . . . 193

B More differential dilepton predictions for AuAu collisions 195 



\section{Chapter 1}

\section{Introduction}

\subsection{Strong-interaction matter under extreme conditions}

Collisions of highly energetic atomic nuclei produce extreme conditions in the laboratory that offer a unique environment to learn about the fundamental aspects of the created matter and its interactions. The conditions are so extreme that the produced matter reaches the, to our current knowledge, highest temperature in the present universe. The densities are equally extreme and only comparable to the center of neutron stars. One of the major motivations to collide heavy nuclei is to create and investigate a new state of matter, which is thought to have also existed shortly after the Big Bang. In this new phase, the nucleons melt and give rise to a plasma of quarks and gluons, the elementary particles of the strong interaction.

The most relevant force for the strongly interacting medium created in heavy-ion collisions is the strong interaction, which is one of the four fundamental forces of nature. The other fundamental forces are gravity, the electromagnetic and the weak force. The strong, electromagnetic and weak force are part of the standard model (of particle physics), which describes the known elementary particles and their interactions. As depicted in Figure 1.1, the elementary fermions (particles with half odd spin) are the quarks (up, down, charm, strange, top, bottom) and the leptons (electron, muon, tau) with their neutrinos counterparts (electron neutrino, muon neutrino, tau neutrino). The gauge bosons (particles with integer spin) carry the forces between them: gluons for the strong, photons for the electromagnetic and $W / Z$ boson for the weak force. The Higgs boson has a unique role as it is responsible for generating the bare mass of the particles. The theoretical framework of the standard model is quantum field theory. The theory describing the strong force is quantum chromodynamics (QCD) (see also Section 1.2.1). The theory of the electromagnetic interaction is described by quantum electrodynamics (QED). QED can be combined with the theory of the weak interaction into a unified theory of the electroweak interaction. These theories are unfortunately notoriously hard to solve. Therefore, studying the predictions, consequences and properties of these theories is a fruitful field of research.

QCD, in particular, has a few remarkable properties: The fundamental degrees of freedoms (quarks and gluons) are not observed freely. Instead, they are bound inside color-neutral ${ }^{1}$

\footnotetext{
${ }^{1}$ Color is the name for the charge of the strong interaction. There are three colors: red, green, blue (plus their respective opposite/anti charges). Either the three different (anti-) colors or color and respective anti-color combine to be colorless.
} 


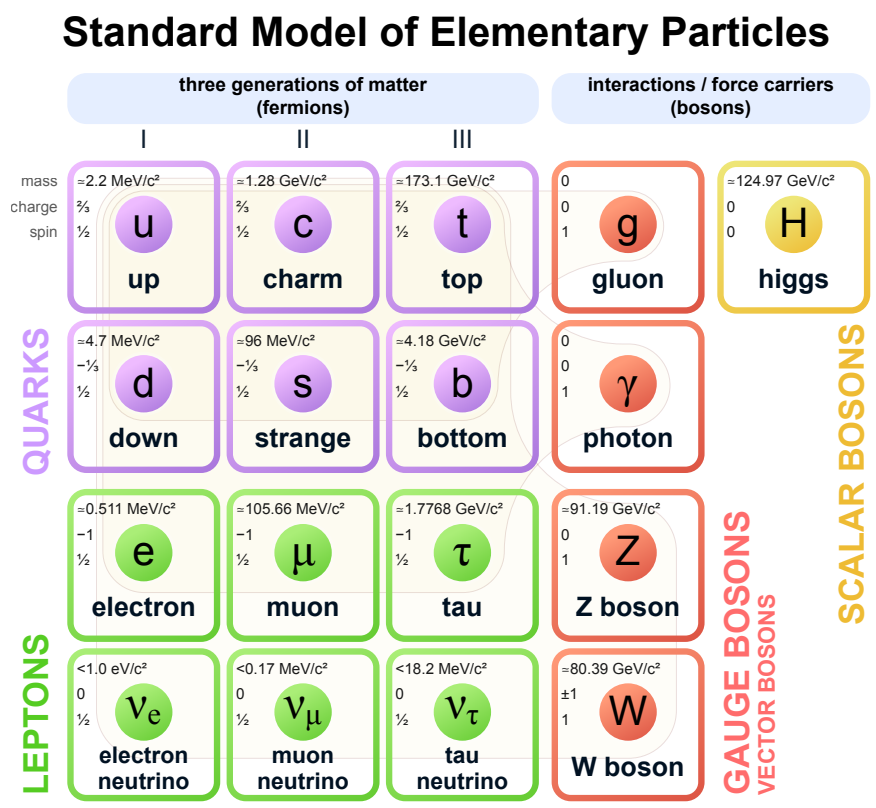

Figure 1.1: Elementary particles of the standard model. Figure from [6].

states called hadrons at all times. This phenomenon is called confinement. The most abundant hadrons are two-quark (mesons) and three-quark states (baryons). More exotic quark states (e.g. tetra- and pentaquark states) are also theoretically possible and a few have been experimentally measured [7,8]. Confinement of quarks is caused by the QCD coupling strength, which is characterized by the coupling constant $\alpha_{S}$. In vacuum, i.e. at low energies, QCD is strongly coupled. The coupling, however, depends on the energy scale (running coupling). As shown on the left of Figure 1.2, the higher the energy i.e. the momentum transfer $Q$ (and the shorter the distance) the weaker the coupling gets. The coupling is said to be asymptotically free ${ }^{2}$. A break-up of hadrons into a soup of free quarks and gluons is therefore expected at high energies, creating a new phase of matter referred to as the quark-gluon plasma (QGP). The theoretical expectation of observing a phase of matter, where partons (another name for quarks and gluons) are confined and a phase, where they are free, is summarized in the QCD phase diagram, drawn in the temperature and baryon-density (or chemical potential) plane (Figure 1.2, right). Of specific interest, is the transition between both phases, which different theoretical predictions (e.g. $[13,14,15,16])$ suggest to be of first order. However, first principle QCD calculations at zero net-baryon density show a cross-over phase transition (see also Section 1.2.1). This limits a first-order phase transition to a certain higher net-baryon density region and suggests the existence of a critical end-point in which the first-order phase transition ends. Another important property of QCD is the spontaneous breaking and restoration of chiral symmetry, which is in detail explained in Section 1.3.1.

Probing the phase diagram and verifying the theoretical predictions of QCD is the goal of the many experimental heavy-ion programs. The energies in the created hot and dense medium are expected to be high enough to produce a quark-gluon plasma. Therefore, particular focus is placed on the search for the quark-gluon plasma phase, the study of

\footnotetext{
${ }^{2}$ For their works on asymptotic freedom [9, 10] David Gross, Frank Wilczek and David Politz were awarded the Nobel prize in 2004.
} 

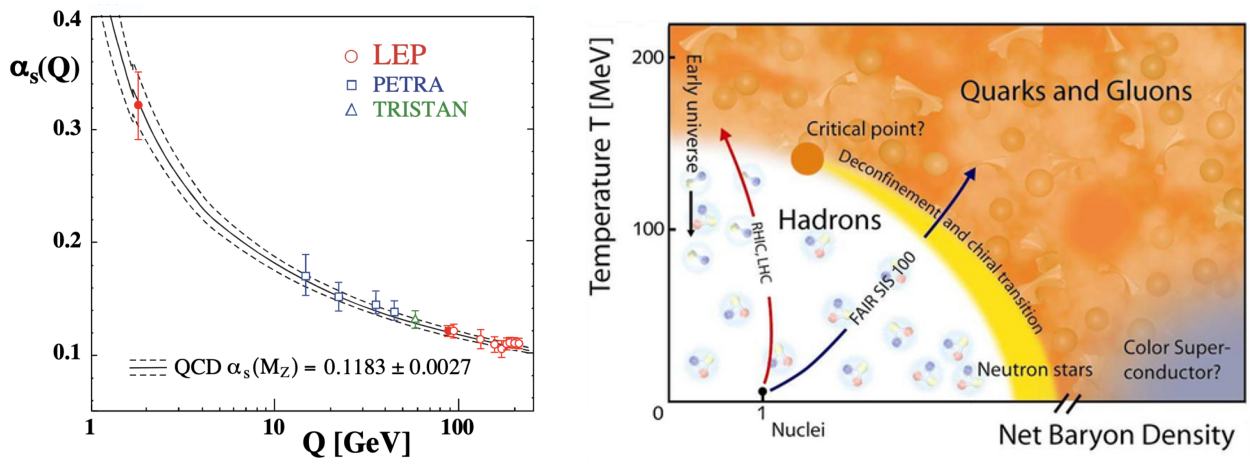

Figure 1.2: Left: Running QCD coupling constant for different momentum transfer $Q$. Figure from [11]. Right: Phase diagram of the strong interaction in the $T$ - $n_{B}$-plane. Figure adapted from [12].

its properties and the discovery of remnants of the phase transition, which so far remains to be experimentally observed. The most relevant high-energy experimental facilities for this work are the Relativistic Heavy Ion Collider (RHIC) at BNL and the Large Hadron Collider (LHC) at CERN [17]. The high incident energies are achieved by using a collider setup that uses two accelerated particle beams. The produced particles are measured by different detectors that are part of large experiments: STAR, PHENIX, PHOBOS and BRAHMS $[18,19]$ in the case of RHIC as well as ATLAS, CMS, LHCb and ALICE [17] at the LHC. The latter being the main heavy-ion experiment dedicated to the study of the quark-gluon plasma [20]. Also, results of the LHC predecessor, the Super Proton Synchrotron (SPS), are discussed in the context of the lepton measurements of the NA60 experiment [21]. Different collision energies probe different trajectories in the phase diagram (as sketched in the right of Figure 1.2). High energy ${ }^{3}$ collisions reach very high temperatures, but only small net-baryon densities, since the colliding nuclei are transparent at ultra-relativistic speeds. At lower beam energies, higher net-baryon densities are reached since the nuclear matter is stopped. A relevant example of a facility for low-beam energy collisions is the SIS18 accelerator at GSI [22]. A fixed-target setup is used at low beam energies experiment since higher luminosities, a measure of the collision frequency, is reached for such a setup. This enables the measurement of high statistics for rare probes, such as leptons. Correlated lepton pairs at SIS18 are measured by the HADES experiment [23] for a wide range of collisions systems. In the search for the phase transition and the critical point, future facilities and experiments, such as FAIR (CBM) [24], NICA [25], JPARC-HI [26] or the RHIC-BES [27], focus on the intermediate energy region to probe a simultaneously hot and dense phase.

One of the most important experimental discoveries so far is the observation of a quarkgluon plasma phase by the different RHIC experiments. The QGP phase is indicated by the compatibility of the measured spectra and flow coefficients with the results of ideal hydrodynamic approaches $[18,19,28,29]$. Surprisingly, the created plasma behaves more like a strongly coupled fluid than a weakly coupled gas [30]. Further evidence of a QGP is provided by the "quenching" of high-pt particles (jets) [31, 32]. As a consequence of the

\footnotetext{
${ }^{3}$ There is no universal classification what a "low", "intermediate" or "high" beam energy is. For this work, "low" beam energies mean $\sqrt{s} \leq 4.5 \mathrm{GeV}$ (as reached by the SIS18 at GSI), "intermediate" beam energies are $\sqrt{s}=5-20 \mathrm{GeV}$ (e.g. RHIC-BES, FAIR or SPS) and "high" beam energies are reached at RHIC and LHC $(\sqrt{s} \geq 130 \mathrm{GeV})$.
} 
RHIC results, a QGP is also expected at LHC experiments, which are performed at even higher energies. At LHC, the last to be experimentally discovered elementary particle, the Higgs boson, was measured in $2012[33,34]^{4}$. A surprising observation at LHC is the measurement of QGP signatures in small proton-proton and proton-lead collision with high multiplicities [38]. The results are consistent with hydrodynamic calculations, which are usually seen as evidence of a QGP phase. As seen from the description of these discoveries, heavy-ion research needs an alliance of theoretical calculations and experimental measurements to advance. The reason is the indirect access of the experimental measurements to the fleeting medium. Since experimental detectors only measure the late remnants of the nucleus-nucleus incident, theoretical calculations are needed to explain how the final particle distribution is produced. In combination with the difficulty of solving first principle theories, testing different assumptions of effective theories in comparison to experimental data is, therefore, one of the major techniques in heavy-ion physics.

The topic of this thesis is the effective theoretical description of the hadron gas stages in heavy-ion collisions. Two avenues of study are explored. First, the emission of correlated lepton pairs and the production of strange hadrons (Section 1.3.1 and 1.3.2) employing only binary reactions are investigated. Second, the theoretical realization and effect of extending the evolution of the hadron gas with multi-particle reactions (Section 1.2.6) are studied, in particular focusing on the resulting deuteron and proton abundances (Section 1.3.3 and 1.3.4). A complete overview of the topics of study is found in Section 1.4.

\subsection{Theoretical description of heavy-ion collisions}

A full dynamical calculation of heavy-ion collisions based on the fundamental theory of quantum chromodynamics is not possible, especially when studying experimentally accessible observables. Effective models, which use input from first principle calculation where possible, are necessary to investigate such dynamic systems. The following sections first introduce the theory of quantum chromodynamics and the different approaches, which aim to extract solutions of it (Section 1.2.1). Then, the different effective approaches that are most relevant for this thesis are detailed. The main approach followed in this work is a (hadronic) transport approach (Section 1.2.4). Also employed are coarse-graining (end of Section 1.2.4) and hydrodynamical (Section 1.2.3) approaches. Thermal models (Section 1.2.2) are not employed, but tension of their predictions with experimental data provides important motivation for the conducted studies. The following compilation hereby assembles the most important approaches for heavy-ion collisions when investigating bulk matter, also referred to as soft particles, which are the low-momentum particles that amount for most of the created medium ${ }^{5}$.

\subsubsection{Quantum Chromodynamics}

Quantum chromodynamics, as the fundamental theory of the strong interaction, describes systems of quarks and gluons as well as their bound states i.e. hadrons. As a quantum field theory, this information is encoded in the Lagrangian, which is given as

$$
\mathcal{L}_{\mathrm{QCD}}=\bar{\psi}_{i}\left(i \gamma^{\mu}\left(D_{\mu}\right)_{i j}-m \delta_{i j}\right) \psi_{j}-\frac{1}{4} G_{\mu \nu}^{a} G_{a}^{\mu \nu} .
$$

\footnotetext{
${ }^{4}$ After the experimental discovery of the Higgs boson, François Englert and Peter Higgs received the Nobel prize for their works on the Higgs mechanism [35, 36, 37].

${ }^{5}$ Absent from this introduction are approaches for high-momentum particles and jets [39], since they are not discussed in this work.
} 
$\psi_{i}$ are the Dirac spinors of the quark fields. The indices of $i$ and $j$ represent color charge $(\mathrm{r}, \mathrm{g}, \mathrm{b}) . \gamma^{\mu}$ are the Dirac matrices, $\left(D_{\mu}\right)_{i j}$ is gauge covariant derivative and $G_{\mu \nu}^{a}$ is the gluon field strength tensor. The index $a$ runs from 1 to 8 . Even though the Lagrangian of QCD and some of the theory's fundamental properties (as explained in the previous section) are known, analyzing results of the theory is complicated and requires evolved methods. Studies are only possible within narrow limits. Most notably, calculating the dynamical evolution of a heavy-ion collision is at the moment far out of reach for firstprinciple calculations.

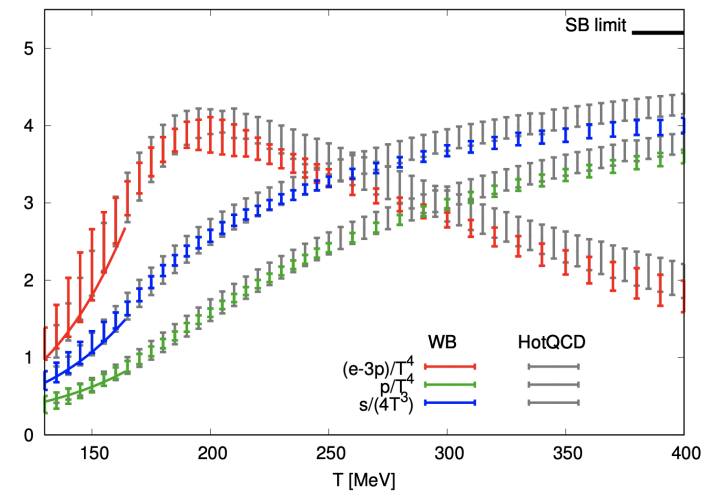

Figure 1.3: Temperature dependence of trace anomaly, entropy density and pressure calculated by Lattice QCD at $\mu_{B}=0$. Figure from [40].

The most established approach for first-principle calculations is Lattice QCD (1QCD, [41]). IQCD calculations non-perturbatively solve QCD by discretizing the space-time (on a lattice) and afterward extrapolating to the continuum. However, due to numerical issues (sign-problem) the results for physical quark masses are limited to a chemical potential of $\mu=0$. Small finite $\mu$ values are accessible e.g. by Taylor expansions around $\mu=$ 0 [42]. IQCD is computationally extremely demanding, such that calculations require state-the-art algorithms and supercomputers to be run. Despite these challenges, it is possible to extract thermodynamic equilibrium properties for strongly interacting quarkgluon systems with this method[40] (see Figure 1.3 for an example). The most striking finding is that of a cross-over phase transition at $\mu \approx 0$ from a hadron gas to a quark gluonplasma [43] at a critical temperature of $T_{C}=155 \mathrm{MeV}$ [44]. Furthermore, 1QCD allows to calculate an equation of state (including the mentioned phase transition) $[45,46,47]$ that is employed as a first principle input for hydrodynamical approaches (see Section 1.2.3).

The main challenge for QCD calculations lies in the nature of the strong coupling constant. Due to asymptotic freedom (as explained in Section 1.1), perturbative methods can only be applied at high energies and short distances. Perturbative QCD calculations cannot access the whole low-energy region, which includes the hadronic sector. They are, however, relevant at high energy scales, which includes the study of jet physics at high momenta [39]. A newer idea is to apply perturbative methods to the in principle non-perturbative strongly-coupled region by finding a dual weakly-coupled gravitational theory to QCD. Such a duality for conformal field theories has already been found, the so called AdS/CFT correspondence [48], and is employed to study aspects of QCD qualitatively [49]. Furthermore, functional approaches exist. Two such non-perturbative field theoretical approaches are the Functional Renormalization Group (FRG, [50]) and the Dyson-Schwinger Equations (DSE, [51]). Both are applicable without the limitation to 
$\mu \approx 0$, but require a truncation of the infinite set of coupled equations, limiting the results' resolution. Alternatively, the different limitations, especially the inaccessibility of the low-energy regime, are circumvented by effective field theories that employ different degrees of freedom, mostly hadrons. The main idea is to rewrite the Lagrangian including the different degrees of freedom in the most general form that still fulfills the symmetries of QCD. The most important symmetry being the chiral symmetry (Chiral Effective Field Theory [52]). A popular example of such a theory is the Nambu-Jona-Lasinio (NJL) model $[53,54]$. The below described Color Glass Condensate (CGC) approaches [55] are also an effective field theory.

An important link between fundamental theory calculations and kinematic approaches as well as experimental measurements is the calculation of cross-sections. A cross-section characterizes the likelihood of two particles interacting. In approaches that model the scattering of partons or hadrons microscopically, it is one of the most important parameters. Quantum field theory approaches, in particular the mentioned chiral effective field theories, are able to derive the transition amplitude (i.e. matrix element $\mathcal{M}$ ) by calculating the scattering $(\mathrm{S})$ matrix for the given Lagrangian. The cross-section is then calculated as follows [56]

$$
\sigma=\int \frac{|\mathcal{M}|^{2}}{4 \sqrt{\left(p_{1} \cdot p_{2}\right)^{2}-m_{1}^{2} m_{2}^{2}}} d \Phi_{n}
$$

with the squared matrix element $|\mathcal{M}|^{2}$ entering in the outgoing differential phase space $d \Phi_{n}$ integration (defined in Eq. 4.15) and momentum $p$ as well as mass $m$ of the incoming particles. Since the underlying theory i.e. QCD is invariant under time reversal (T symmetry), the matrix element for the forward and backward process $(a \leftrightarrow b)$ has to be the same:

$$
\left|\mathcal{M}_{a \rightarrow b}\right|^{2}=\left|\mathcal{M}_{b \rightarrow a}\right|^{2} .
$$

The microscopic consequence of this relation is that the number of processes $a \rightarrow b$ and $b \rightarrow a$ have to be equal in thermal equilibrium. This is called the principle of detailed balance and a critical requirement for kinematic approaches.

\subsubsection{Thermal Models}

A simple, but very successful class of effective models are so called statistical hadronization models (SHM), sometimes also referred to as statistical or thermal models [57]. The assumption is that the medium created in a heavy-ion collision is in full thermal and chemical equilibrium at one point. The system freezes out chemically during this time according to the thermodynamic expectation of the grand-canonical ensemble ${ }^{6}$. The chemical composition of the emitted hadronic medium can therefore be determined by calculating the particle number $N$ of species $i$ according to

$$
N_{i}=\frac{g_{i} V}{2 \pi^{2}} \int_{0}^{\infty} \frac{p^{2} d p}{e^{\left(E_{i}-\mu_{i}\right) / T} \pm 1},
$$

where $g_{i}$ is the spin degeneracy and the \pm is - for bosons and + for fermions. With this assumption a temperature $(T)$ and chemical potential $(\mu)$ are extracted from the experimentally measured particle numbers by a common 3-parameter fit in $T, \mu, V$ for all particles. $V$ is the fireball volume at freeze-out and can be excluded from the fit, when considering particle ratios, where it cancels.

\footnotetext{
${ }^{6}$ Depending on the beam energy and collision system also the canonical expectation is employed [58].
} 
Measured particle multiplicities and multiplicity ratios are very successfully reproduced for a wide range of incident energies and systems by finding one $(T, \mu, V)$-set for a given experiment [59, 60, 61, 62, 63], which suggests that the medium close to chemical freeze-out is thermalized. If the thermal models result from different experiments are combined, they

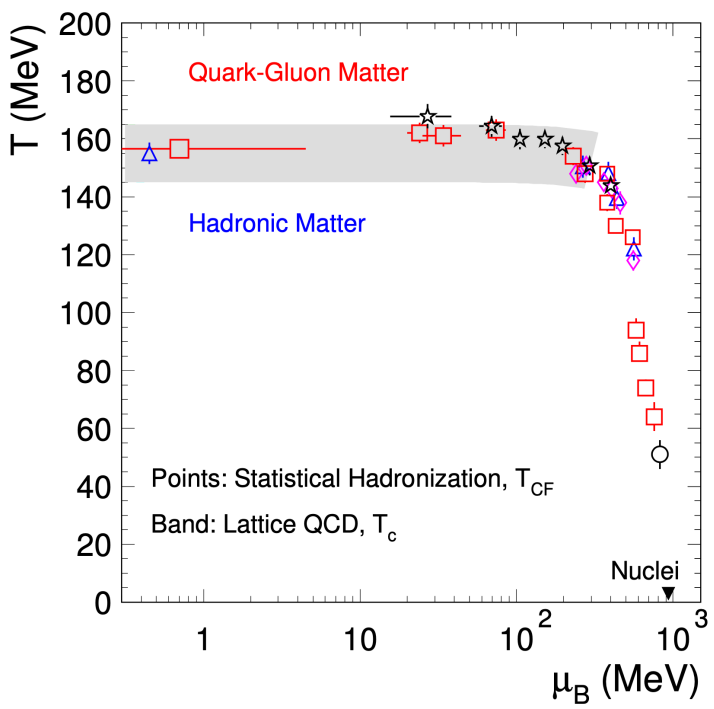

Figure 1.4: Comparison of Lattice QCD calculations for the critical temperature $\left(T_{C}\right)$ of the phase transition (band) and the fitted temperature $\left(T_{C F}\right)$ and baryon chemical potential for different experiments from thermal models (symbols). Taken from FIG.5 in [63]. See caption in [63] for references to calculations.

agree with the cross-over phase transition region mapped out by Lattice QCD calculations in the $T$ - $\mu_{B}$-plane for the high-beam energy experiments (Figure 1.4, $[62,63]$ ). For highenergy experiments, the temperature is close to $T_{C} \approx 155 \mathrm{MeV}$, the critical temperature measured by Lattice QCD. This finding is consistent with an immediate chemical freezeout after the phase transition to hadronic matter. The results also qualitatively follow the phase transition line assumed for the strong interaction (Figure 1.2).

Not all observables are correctly described or can be calculated with thermal models, particularly observables that differentially probe the momentum space. While an extension with so called Blast-Wave models [64, 65] is possible in order to describe transverse momentum and rapidity spectra, no agreement is generally reached e.g for higher flow coefficients $\left(v_{2}, v_{3}, v_{4}, \ldots\right)$. Blast-Wave models introduce an additional expansion rate of the thermal source characterized by a flow velocity $\beta$. Furthermore, the assumptions of thermal models rule out the possibility of any non-equilibrium effects in the late stages like inelastic rescattering that would change the chemical composition of the system. However, serval results $[66,67]$ show that non-equilibrium effects in the late stages of a collision are significant for identified particle spectra or correlation and fluctuation observables.

Even though thermal models are not directly employed in this work, the disagreement of their fit with the measured proton yield at LHC [61] and the double strange baryon yields of $\phi$ and $\Xi$ yield measured by HADES [68] partly motivate the studies in this thesis. The explored explanation for the observed discrepancies is provided by accounting for non-equilibrium effects with a hadronic transport approach in the late collision stages. Furthermore, an agreement for the production of light nuclei is observed with the thermal 
model $[69,70]$, which leads to the question of how such nuclei should survive after freezeout if the surrounding temperature is orders of magnitudes larger than the binding energy (snowballs in hell). More details on this work's study of these discrepancies and questions are given in Section 1.3.

\subsubsection{Hydrodynamics}

Hydrodynamical approaches are employed, since the QGP produced in heavy-ion collision for a certain time is found to be an almost perfect fluid [30]. Essentially, hydrodynamic approaches calculate the macroscopic evolution of (thermodynamic) quantities of the system, when enforcing conservation of energy, momentum and charge(s) [71]. The main equations of relativistic hydrodynamics are therefore formulated as follows:

$$
\begin{gathered}
\partial_{\mu} T^{\mu \nu}=0, \\
\partial_{\mu} j_{i}^{\mu}=0,
\end{gathered}
$$

where $j_{i}^{\mu}$ is the current of the conserved charge i (e.g. of baryon number or electric charge) and $T^{\mu \nu}$ the energy-momentum tensor.

Employing hydrodynamics is possible under two assumptions. First, the system needs to be "macroscopic" so that a fluid description can be applied. This is the case when the mean-free path of the interacting particles is much smaller than the size of the system. Second, the system needs to be close to local thermal equilibrium. Both assumptions are expected to be fulfilled soon after the impact of the nuclei in a heavy-ion collision. How the system thermalizes so rapidly, however, is still unknown $[72,38]$. Interestingly, a hydrodynamic description is successful even for elementary proton-proton reactions if the event produces a large multiplicity [73].

The set of equations above is not closed without one additional equation, the equation of state. It relates the pressure $(p)$ with energy $(\epsilon)$ and charge $(n)$ density.

$$
p=p(\epsilon, n)
$$

The equation of state encodes all information and properties of the medium since the other equations are just expressions of conservation laws. This causes two advantages of hydrodynamical approaches: (i) the equation of state can encode a phase transition from QGP to hadron gas and (ii) it is possible to employ an equation of state calculated from first principles by Lattice QCD [74]. Especially, the inclusion of a phase transition is challenging in microscopic approaches.

In the ideal case, hydrodynamic approaches are limited to systems in full local thermal equilibrium. The extension to viscous hydrodynamics can account for small dissipative effects $[75,76,72]$. The applicability is furthermore extended to highly anisotropic system by (anisotropic hydro,[77]), where the viscous expansion is done for an already anisotropic distribution. In the viscous hydrodynamics formulation, additional parameters appear that describe the relaxation of the medium back to equilibrium, so called transport coefficients. A new development is to employ modern mathematical statistics tools (Bayesian analysis) to constrain those coefficients systematically [78].

The assumption of (local) equilibrium is not fulfilled in the initial nucleon collisions. Therefore different approaches are employed to provide a close-to-equilibrium initial state of the 
energy-momentum tensor as the hydrodynamic calculation starting point. This initial energy distribution is either smooth by averaging over events or lumpy to study event-byevent fluctuations. The most important initial state approaches can be divided into three categories [79, 80]: Glauber, color glass condensate and transport approaches. All three categories usually employ Monte-Carlo methods. Glauber models [81] assume a WoodsSaxon distribution for the distribution of the nucleons inside the nucleus and a geometric picture of how the nucleons collide on impact. The color glass condensate (CGC) models are based on the idea that in relativistic collisions, the parton distribution function of the nucleons is initially saturated by gluons since small Bjorken- $x$ values are probed. The collision of the nuclei is therefore governed as the interaction of the color fields produced by the gluon distributions in the nucleus. Popular CGC-based approaches are IP-Glasma [82] and MC-KLN (Monte-Carlo Kharzeev-Levin-Nardi) approaches [83, 84]. The last category is marked by partonic or hadronic microscopic transport approaches, like SMASH [85], UrQMD [86], EPOS [87] or AMPT [88], where the parton or hadron distributions are converted into the needed energy, baryon and charge density distribution after the initial scatterings.

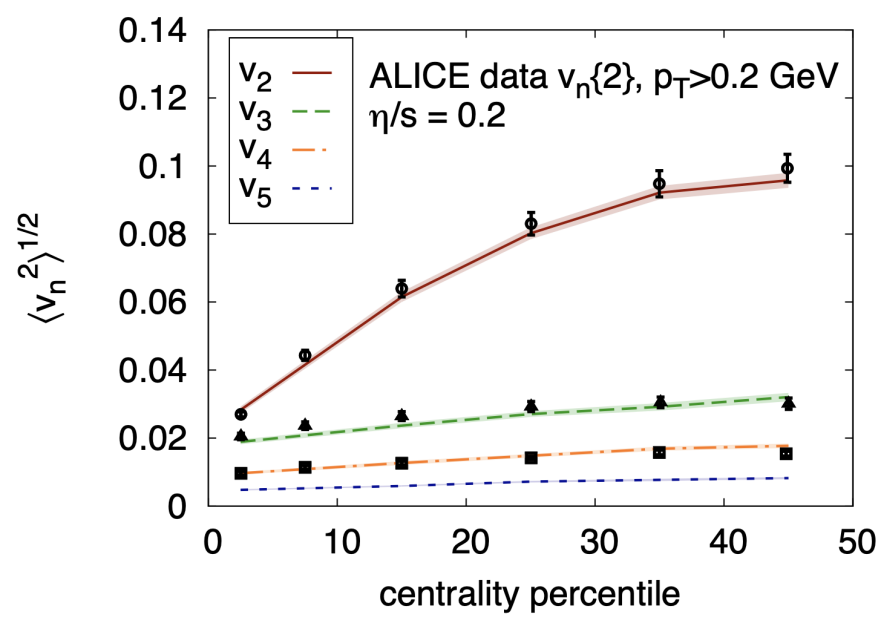

Figure 1.5: Anisotropic flow coefficients from a hydro dynamical approach paired with CGC initial state model compared to LHC data. Result from [89].

The hydrodynamic calculation evolves the initial anisotropy in coordinate space, provided by the initial state approach, into an anisotropy in momentum space. This is, for example, important to match the experimentally measured elliptic flow [79]. In general, hydrodynamic approaches are remarkably successful in describing the measured bulk matter data (e.g.[89] and Figure 1.5). In order to compare to identified particle spectra from experiments, the evolved energy-momentum tensor is converted to microscopic hadronic degrees of freedom by the Cooper-Frye formalism [90]. This conversion is done when the assumptions of hydrodynamics (local equilibrium, small mean-free path) break down.

Examples of popular hydrodynamical approaches are vHLLE [91], MUSIC [92, 93] and CLVisc [94]. In the following, MUSIC (Section 5.3) and vHLLE (Section 5.4) are employed as part of a hybrid approach (Section 1.2.5) in order to calculate the evolution of the hot and dense matter close to equilibrium in intermediate and high energy collisions. 


\subsubsection{Transport approaches}

Transport approaches are a microscopic many-body description of a medium and numerically solve the Boltzmann equation, sometimes also referred to as the Boltzmann transport equation (Eq. 1.8). It is the fundamental equation of what is referred to as kinetic theory. Most importantly, the described system is not required to be in equilibrium, so non-equilibrium dynamics can also be studied. The nonlinear integro-differential equation is not analytically solvable in general. For some special cases analytical solutions exist though (cf. Figure 2.3 and related discussion for an example). The classic ${ }^{7}$ Boltzmann equation reads

$$
\frac{\partial f}{\partial t}+\frac{\vec{p}}{m} \nabla f+\vec{F} \frac{\partial f}{\partial \vec{p}}=\left(\frac{\partial f}{\partial t}\right)_{\text {coll }},
$$

where $f=f(\vec{r}, \vec{p}, t)$ is the phase-space probability density function, meaning that the number of particle inside a differential phase space volume $d^{3} \vec{r} d^{3} \vec{p} /(2 \pi)^{3}$ is given as $d N=f(\vec{r}, \vec{p}, t) d^{3} \vec{r} d^{3} \vec{p} /(2 \pi)^{3}$. The right hand side is called the collision integral and is in detail discussed in Section 4.1. The equation is only applicable for dilute (enough) systems, where dilute means that the mean-free path needs to be significantly larger than the Compton wavelength $\left(\lambda_{C}=\frac{h}{m c}\right)$. This limitation stems from the derivation of the Boltzmann equation from the more fundamental BBGKY hierarchy [95]. When modeling the Boltzmann equation in numerical Monte-Carlo (transport) approaches, the different particles are propagated according to equation of motions that are equivalent to the left-hand side of 1.8. The collision term is modeled by different interactions between the particles. See Section 2.2 in Chapter 2 for an example of such a prescription. Two categories for transport approaches exist [96]: the Boltzmann-Uehling-Uhlenbeck (BUU) and the quantum molecular dynamics (QMD) approach. The two differ in the representation of the phase-space distribution. BUU approaches use many test particles to model the distribution, while QMD-like approaches use a Gaussian wave packet to signify the particle wave function. This difference is only relevant when incorporating (mean-field) potentials and particle correlations [97]. Different degrees of freedom are chosen for transport approaches

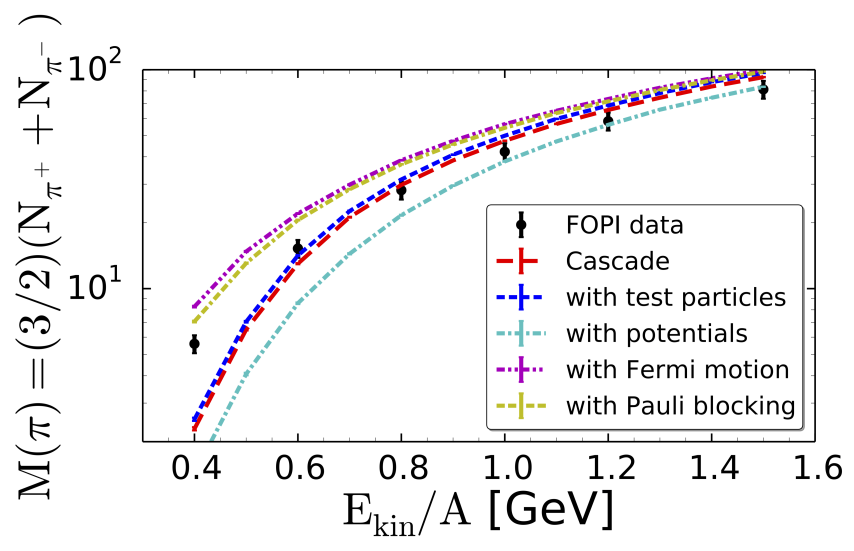

Figure 1.6: Pion multiplicity for low kinetic beam energy AuAu collision of a transport approach with different features (see Chapter 2 for more details). Result from [98].

in the context of theoretical heavy-ion collisions. Approaches that aim to describe the hot

\footnotetext{
${ }^{7}$ Note that a relativistic formulation is easily attainable.
} 
and dense stage beyond the phase transition employ quarks and gluons (partonic transport approaches). For the stages of heavy-ion collisions, where no quark-gluon plasma has formed, the degrees of freedom are hadrons (hadronic transport approaches). Some approaches also include both phases.

Hadronic transport approaches have a long history [99, 100, 101, 102, 103] of being employed to model nucleus-nucleus collisions since the beam energies of older experiments are (according to today's standards) low and not sufficient to produce a quark-gluon plasma. Hadronic transport approaches were instrumental in the discovery of QGP signatures: Since the results at AGS (Alternating Gradient Synchrotron, the predecessor of the RHIC) could be reproduced by transport models, it was concluded that no QGP signatures are seen in the experimental data [104]. While the inability of transport approaches to reproduce the strangeness measurement at SPS (see also Section 1.3.2), was seen as a clear indication of a QGP formation [105]. Also, for current high beam energy collisions, hadronic transport approaches are still applied as part of state-of-the-art hybrid approaches (Section 1.2.5) for the hadron gas phases. Additionally, they remain relevant for low beam energies, as lower beam energies are again being focused on experimentally to produce highly dense systems. An example of a successful application of a hadronic transport approach at low energies is displayed in Figure 1.6. The pion excitation function at low beam energies is comparable to the experimental measurements.

The hadronic transport approach SMASH [85] is employed in this thesis as the main theoretical approach (see Chapter 2) for all conducted studies of the hadron gas stages of heavy-ion collisions. These stages include the initial and final phase of high-energy and the whole evolution of low-energy collisions, where no phase transition is expected. Other transport approaches that are successfully employed and still under active development include UrQMD [106], (P)HSD [107, 108], GiBUU [109], JAM [110], BAMPS [111] and AMPT [112]. For a recent comparison of the different approaches, the reader is referred to [113].

\section{Coarse-Graining}

Approaches that connect microscopic transport approaches to the macroscopic observables available in hydrodynamic calculations are called coarse-graining approaches [114]. They convert the propagated particles into a density distribution in macroscopic grid cells. For those cells, the temperature $(T)$ and baryon chemical potential $\left(\mu_{B}\right)$ are calculated using an interchangeable equation of states from the energy and baryon density of the cells. Coarse-graining approaches thereby allow to compare macroscopic observables resulting from transport and hydro approaches [114] and to employ (medium-modified) thermal emission rates for photons and dileptons $[114,115,116,117,118]$, which require $T$ and $\mu_{B}$ as an input. Results for such a thermal emission of dileptons are presented in Chapter 3. The employed approach is detailed in Section 2.4.

\subsubsection{Hybrid models}

As explained in the previous sections, different kinematic approaches have different conditions of applicability. These conditions are expressed as a requirement on the mean-free path. For transport models, the mean-free path needs to be large i.e. significantly larger than the Compton wavelength and for hydrodynamic models, the mean-free path needs to be small i.e. much smaller than the size of the system. Consequently, a hydrodynamical description is valid for high densities and transport approaches for dilute systems. There- 
fore, a combination of approaches (a hybrid model) is employed [119, 120, 121, 122] to map the different models to an appropriate stage of a heavy-ion collision. Since pure QCD

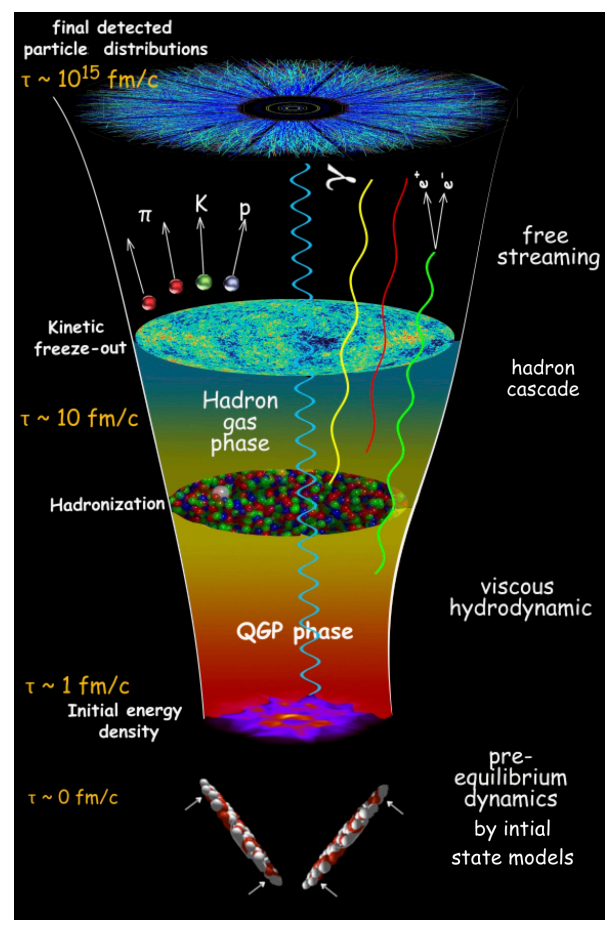

Figure 1.7: Stages of a heavy-ion collision. Models employed at different stages depicted on the right. (adapted from [123])

calculations are not applicable (see Section 1.2.1 above), this is currently the "standard model" for the evolution of the medium and description of the final state measured in the experimental detectors at intermediate to high beam energies. QCD calculations are used as an input where possible.

Hybrid models also separate the collision medium into areas and stages close to and far from equilibrium, which is necessary due to the equilibrium requirement of hydrodynamic approaches. This assumption is slightly relaxed for viscous hydrodynamic approaches that require a medium only close to equilibrium. The combination of approaches is therefore built as follows (see Figure 1.7 for an illustration). The two colliding nuclei are converted into an initial energy density by the introduced initial state models above (Glauber, CGC, transport, ...). They are applied to describe the initial nucleon scatterings in the far from equilibrium early stage of the collision. For the following hot and dense stage, the medium is expected to be close to equilibrium. Therefore, the hydrodynamic approach is employed starting from the calculated initial energy density. Most importantly, this approach can include an equation of state calculated from Lattice QCD, which encodes a cross-over phase transition. In high energy collisions, the initial degrees of freedom are quarks and gluons, described by the state equation at high temperatures and densities. As the medium cools but hydrodynamics remains applicable, the degrees of freedom become those of a hadron gas. The ability to account for a phase transition is one of the main advantages of hybrid approaches. The hydrodynamic approach furthermore relies on the additional input of transport coefficients. Comparing the hybrid approach results for different coefficients with experimental data enables to constrain them systematically [124, 78]. As the system expands and evolves out of equilibrium, the assumption of hydrodynamics breaks down and 
hadronic transport models are employed (as a so called afterburner). The macroscopic hydrodynamic evolution is converted (particilized) into hadronic degrees of freedom by the Cooper-Frye formalism [90]. A hadronic transport cascade is applied for the late nonequilibrium rescattering phase since otherwise final particle spectra are not matched [66, 67 .

Hybrid approaches are exceptionally successful at high beam energies as probed at RHIC and LHC [122], which is why they are considered the "standard model". However, some limitations are attached, mainly connected to the interface between the different approaches: 1) An ideal form of the energy-momentum tensor is assumed at initialization of the hydrodynamic approach. 2) The particlization process by the Cooper-Frye formalism does not account for negative contributions i.e. transport particles cannot feedback into the hydrodynamic calculation. 3) The condition when to particilize i.e. switch from hydrodynamic to transport approach, is a free parameter of the approach. The effects of these limitations are largely unclear and could be studied by a more dynamic coupling of transport and hydrodynamic approach.

As mentioned, some transport approaches also cover the entire evolution at high energies as an alternative approach to hybrid models $[108,112]$. While it is common at low beam energies to apply transport models to the entire evolution, at high energies accounting for the changing degrees of freedom in the phase transition arises as a major challenge for such approaches.

Hybrid approaches are employed in this work for the deuteron and proton studies in Section 5.3 and Section 5.4, where they are also described.

\subsubsection{Multi-particle reactions}

Multi-particle reactions are defined as all reactions with more than two particles in initial or final states. They gain importance with higher density descriptions. Therefore, accounting for them in a theoretical (transport) description is relevant when considering the highly compressed matter produced in heavy-ion collisions. In particular, as future experiments explore high-density collisions at intermediate energies. Multiple experimental programs are in preparation or ongoing at these energies: the RHIC beam energy scan, FAIR, NICA and JPARC-HI. All with the major goal to discover signatures of the critical point or a (first-order) phase transition. In order to distinguish and constrain such experimental signatures, it is necessary to employ accurate models for the high-density hadron gas stage. While it is natural to employ hydrodynamic approaches at high density, they require the strong assumption of local equilibrium. An alternative is to alleviate the restriction to binary reactions of transport approaches and extend them to include multi-particle reactions. In this way, the applicability range is extended towards higher densities ${ }^{8}$ and the hydro regime, but without an assumption about equilibrium. Multi-particle reactions are closely connected to the process of a (fast) equilibration and thermalization [111]. For example, in [125], it is shown that when introducing effective N-particle reactions by forcing local thermalization in hadronic transport, the solution of the transport calculation gets closer to the hydrodynamic solution.

Different multi-particle reactions are known to be important, independently of the density. For some reactions only the relevance of the binary forward reaction is known and the

\footnotetext{
${ }^{8}$ Note that even with multi-particle reactions, transport approaches are still limited to densities, where the effect of quantum interference is negligible.
} 
relevance of the multi-particle backward reaction is an open question (see Section 1.3.4). Examples, where the importance of multi-particle reactions has been demonstrated in the literature, are:

- Deuteron catalysis: $N p n \leftrightarrow N d$ and $N p n \leftrightarrow N d$ in $[126,127]$.

- Baryon-anti-baryon annihilation: $B \bar{B} \leftrightarrow 3$ mesons in $[128,129,130]$

- Gluon Bremsstrahlung: $g g \leftrightarrow g g g$ in [111].

All studies employ transport approaches, which account for the multi-particle reactions microscopically. Usually, transport approaches neglect multi-particle reactions, even though it is theoretically attractive to employ them since they are numerically challenging to account for. Without multi-particle back-reactions, detailed balance is broken for all reactions that produce more than two particles like $1 \rightarrow n$ decays or $2 \rightarrow n$ scatterings $(n>2)$. The reactions are not able to equilibrate. This conflicts with the time-reversal invariance, which is strictly fulfilled for the strong interaction. Neglecting such back-reactions is, therefore, a common criticism and point of debate regarding calculation results [131, 132]. Also, the extraction of switching temperatures [133] and transport coefficients [78] in hybrid approaches rely on a correct approach for the hadronic reaction dynamics in the late collisions stages since final particle yields are used as a constraint.

The theoretically most rigorous approach to include multi-particle reactions in transport models is a stochastic collision criterion $[134,126]^{9}$, which is directly based on collision probabilities derived from the collision term of the Boltzmann equation. It is inherently boost-invariant and, most importantly, straightforward to extend to arbitrary n-to-m collisions. Alternatively, multi-particle reactions are modeled using a chain of binary reactions. This approach is more limited in scope since it relies on appropriate intermediate resonance states and introduces an artificial reaction time with unknown side effects. If no appropriate intermediate resonance state exists, it has to be artificially introduced [135]. Employing the stochastic criterion avoids such numerical artifacts by treating multi-particle reactions directly and, therefore, can also constrain their side-effects.

In this work, multi-particle reactions known to be important for specific particles are studied. The three reaction classes are 3-to-1, 3-to-2 and 5-to-2 reactions. For this, the stochastic criterion is first theoretically derived and then introduced in a hadronic transport approach. The multi-particle treatment is then employed to investigate the relevance of the Dalitz decay back-reaction in low to intermediate beam energies, the $\mathrm{d}$ production via catalysis reactions in the afterburner stage at intermediate beam energy and the regeneration of $p \bar{p}$ pairs lost by annihilation reactions at high energies (Section 5.2 to 5.4). The motivation behind these studies is discussed in the following sections.

\subsection{Observables of interest}

\subsubsection{Chiral symmetry and dilepton production}

One of QCD's most fundamental symmetries is the chiral symmetry for massless fermions i.e. quarks, which entails that the right or left "handedness" (chirality) is conserved (see [136] for a topical review). Since the quarks are not massless and carry a bare mass, the symmetry is explicitly broken. However, since the masses of the lightest quarks are small, the chiral symmetry is considered approximately fulfilled for them. More interestingly,

\footnotetext{
${ }^{9}$ often referred to as the local ensemble method
} 
chiral symmetry is also spontaneously broken ${ }^{10}$. i.e. the Lagrangian is invariant under chiral transformation, but the solution (ground state) is not. This breaking is caused by the quark-antiquark condensate and generates the masses of hadrons, which are significantly larger than their constituent bare quark masses [137]. A manifestation of the spontaneous breaking is found in the observed hadron spectrum, where chiral partners e.g. $\rho$ and $a_{1}$ are not degenerate. For high energy densities, the chiral symmetry is expected to be restored and the quark-antiquark condensate to vanish. This symmetry transition coincides with the deconfinement phase transition according to Lattice QCD calculation at zero chemical potential [40], even though they are in principle conceptually distinct. The quark-gluon plasma, therefore, is expected to be chirally symmetric.
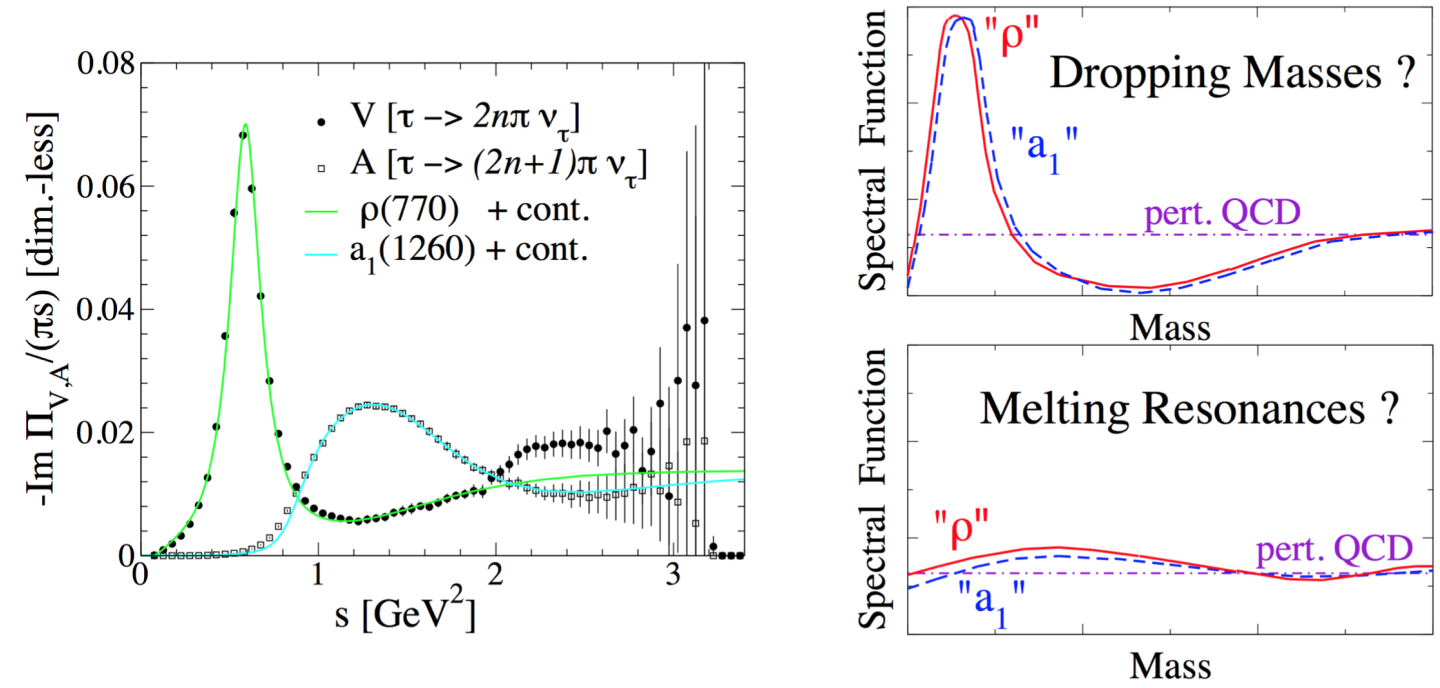

Figure 1.8: Left: Measurements of the $\rho$ and $a_{1}$ spectral functions. Right: Sketched scenarios of spectral function changes under chiral symmetry restoration. Results from [136].

If chiral symmetry is restored, the chiral partners $\rho$ and $a_{1}$ become degenerate i.e. their mass distribution is matching. Note that this is not the case in vacuum, as experimental measurements (on the left in Figure 1.8) show. A modification of the spectral function indicating a restoration of chiral symmetry inside a hot and dense medium has been discussed in the literature extensively $[138,139,140,141,136,142,143]$. Historically, two modification scenarios were prevalent, in particular for the $\rho$ meson: a shift in mass or a broadening of the spectral functions $[144,145]$ (cf. right plot in Figure 1.8). For example, calculations based on hadronic many-body theory [145] and the functional renormalization group [146] are performed to obtain a quantitative understanding of this effect.

Dileptons are correlated pairs of lepton-antilepton pairs. In heavy-ion collisions, only the production of electron and muon pairs are relevant since the tau lepton is (too) heavy and short-lived. The lepton pairs offer a clean probe for hot and dense matter. Since they only interact electromagnetically, they escape the medium nearly unperturbed, thus allowing unique access to the properties of the strongly interacting medium itself and resonances that decay within it. In contrast, hadronic decay products suffer from rescattering and absorption such that the interesting information about the hot and dense stage is masked. Therefore, dileptons are considered one of the most interesting observables.

\footnotetext{
${ }^{10}$ Yoichiro Nambu received the Nobel prize in 2008 for his description of the spontaneous breaking of chiral symmetry of the strong interaction.
} 
In the context of chiral symmetry and its restoration, the main dilepton source of interest are direct decays of vector mesons, e.g. $\rho \rightarrow e^{+} e^{-}$. The invariant mass spectrum of the produced dileptons reflects the decaying resonance mass distribution. While a whole cocktail of sources (described below) contributes to the total dilepton spectrum, around the $\rho$ pole mass, theoretical calculations (e.g. [85]) predict the $\rho$ contribution to be dominant. Therefore, the dilepton invariant mass spectrum allows direct access to the spectral function inside the hot and dense medium.

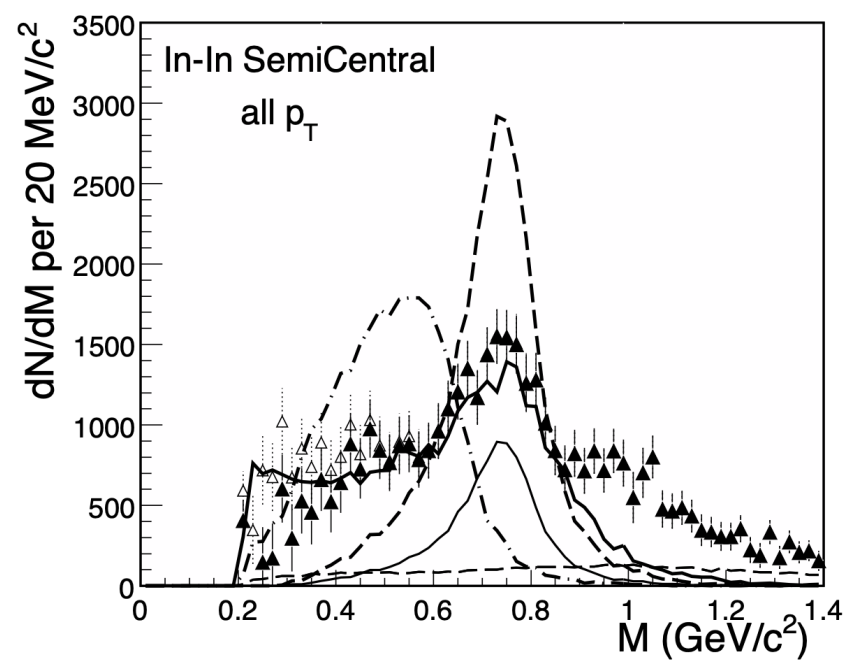

Figure 1.9: NA60 excess dimuon mass spectrum with different $\rho$ spectral modification: no modification (thick dashed), mass-shift (dash-dotted), broadening (solid). Result from [147].

Experimentally, the emission of dielectrons and dimuons was studied at several different facilities. At CERN SPS dimuon production in indium-indium collisions was recorded by NA60 [148, 147]. The high-quality experimental data allowed the investigation of the $\rho$ spectral function and confirmed the previous dilepton measurement from CERES [149] that revealed an excess in the low invariant mass region. The findings of NA60 essentially settled the debate of how the $\rho$ spectral shape changes inside a hot and dense medium. As seen in Figure 1.9, the data is consistent with a broadening and disfavor a mass shift of the $\rho$ [150]. For higher energies up to $\sqrt{s_{N N}}=200 \mathrm{GeV}$, dileptons are measured at RHIC by STAR [151] and PHENIX [152]. Both also report an enhancement in the dilepton invariant mass range from 0.30 to $0.76 \mathrm{GeV}$, which is again attributed to a broadening of the $\rho$ spectral function. The observed broadening is one of the only experimental indications of chiral symmetry restoration.

The present work focuses on the dielectron production in the kinematic regime of beam energies of $E_{\mathrm{Kin}}=1-3.5 \mathrm{AGeV}$, which is covered by the HADES experiment [153, 154, $155,156,157,158,159]$ at the GSI facility. The HADES results confirmed previous measurements from the DLS collaboration [160]. In the future, the CBM experiment at FAIR [22] will add to the existing experimental data with new results from the intermediate energy range, specifically probing the dilepton emission from the high net baryon density region, together with complementary programs from NICA and J-PARC.

The dominant and relevant sources at low beam energies and low invariant masses up to about $1 \mathrm{GeV}$ are decays of various resonances. The Dalitz decays of the pseudo-scalar 
mesons $\pi$ and $\eta$ plus the $\omega$ Dalitz decay dominate for the lowest masses. The direct decays of the vector mesons $\rho, \omega$ and $\phi$ show up as peaks at the respective pole mass in the spectrum. The different sources are, in general, roughly separated by the invariant mass region of the produced pairs. For high energy measurements, direct decays of the heavier $J / \Psi$ and the higher charmonium state $\Psi^{\prime}$ show up as peaks. If $c$ and $\bar{c}$ pairs do not form a charmonium state, but rather a $D$ meson with a lighter quark and those $D \bar{D}$ pairs decay into an (anti)lepton separately, They are also correlated despite the intermediate steps. Open charm decays form a dominant contribution from 1 to $2.5 \mathrm{GeV}$. Similarly, $b \bar{b}$ pairs that form $B$ mesons are relevant for the high-mass region above $4 \mathrm{GeV}$. For the same mass region, the Drell-Yan-Process is contributing, where during the primary hard scatterings of hadrons, two quarks from separated hadrons annihilate into a virtual photon that subsequently decays into a dilepton. Another source is the annihilation from $q \bar{q}$ pairs inside the QGP where the quarks are not bound. This source is only dominant at higher beam-energies in the intermediate-mass region. An isolation of this thermal contribution from the background by subtracting other contributions (resonance and open charm decays) in the low or intermediate mass region would offer unique experimental access to extract a (time-integrated) temperature of the hot and dense medium. This temperature would not suffer from a blue shift, which for example, photon momentum spectra do. Fits of the dilepton invariant mass spectrum for large collision systems are also performed at lower energies, where no QGP is expected to form, to extract a timeintegrated temperature [161].

In order to connect the theoretical calculations of the vector meson spectral functions with experimental measurements and to help disentangle the different dilepton cocktail sources, dynamical approaches that describe the evolution of heavy-ion collisions in detail have to be employed. In this work, the dilepton emission is studied within a hadronic transport approach, which is comparable to previous work using the GiBUU [85], the HSD [162] and the UrQMD [163] approach. These works cover a variety of aspects concerning the dilepton production at low energies, such as the effect of the coupling of the $\rho$ meson to baryonic resonances, Bremsstrahlung, the $\Delta$ contribution and the density dependence.

The employed hadronic transport approach includes the dilepton emission from the dominant sources at low beam energies, which is are dilepton decays of various resonances (see Section 2.4 for details). The results (Section 3.1) are systematically confronted with the complete set of HADES dielectron data for elementary, proton-nucleus and nucleusnucleus reactions at low beam energies. The resonance treatment based on vacuum properties allows to establish a well-understood baseline in elementary and small systems. To investigate medium effects on the dilepton emission, a coarse-graining approach based on the same hadronic evolution is employed, which includes in-medium modifications to the vector meson spectral functions. The dilepton experimental data set also provides a rich testing ground spanning from elementary to heavy-ion collision for the evolution of the transport approach (with only binary collisions) itself.

\subsubsection{Strangeness production}

The yields of different hadron species are the basic observable to study hot and dense matter properties and the particle production mechanisms. Since strange quarks (i.e. the strangeness quantum number) are not present in the nuclei before the collision, they have to be newly produced in order to form the measured strange hadrons. Even though rescatterings might perturb the formed hadrons in the medium after their production, the strangeness is generated by and therefore a messenger of the medium itself. Of particular 
interest is the so called sub-threshold production. If the beam energy is not sufficient for a direct production in the $N N$ scatterings, higher mass strange hadrons can only be produced in secondary reactions, which makes them even more sensitive to the medium dynamic and properties [164].

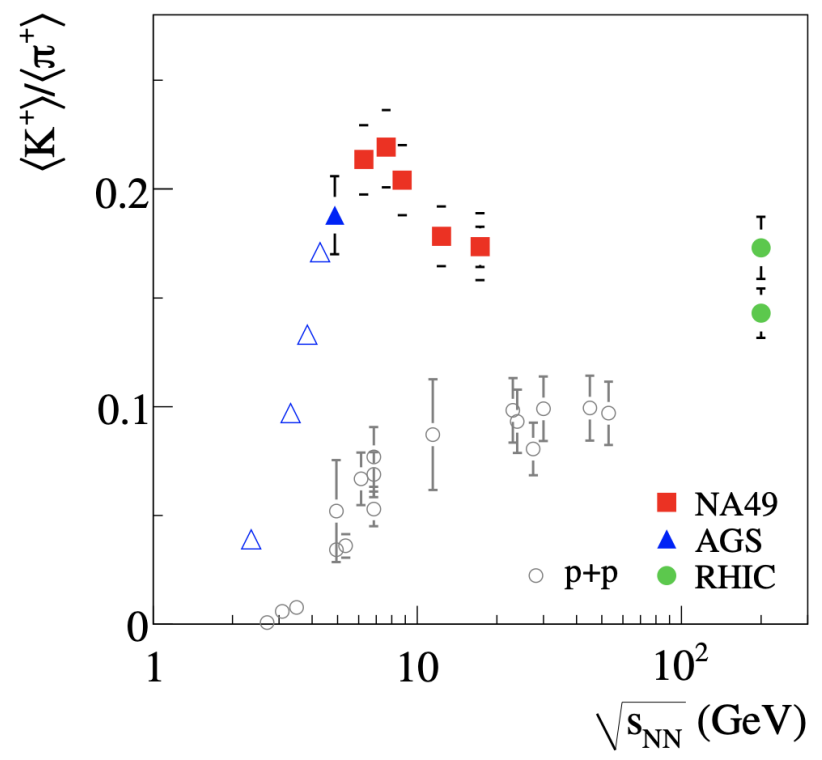

Figure 1.10: Energy dependence of the $\langle\mathrm{K}+\rangle /\langle\pi+\rangle$ ratio measured in central $\mathrm{Pb}+\mathrm{Pb}$ and $\mathrm{Au}+\mathrm{Au}$ collisions (colored symbols) versus elementary pp results for the same energy (uncolored symbols). Result from [165].

The main research interest regarding strangeness production stems from the historical idea that strangeness is enhanced in a collision when a quark-gluon plasma is formed [166]. Indeed, experimentally the strangeness production is found to be enhanced, in particular of strange antibaryons, in nucleus-nucleus reactions compared to elementary reactions, which is explained by the presence of a QGP phase [167]. Among the most famous enhancement measurements seen as a QGP signature is the "horn" in the $K^{+} / \pi^{+}$ratio measured by NA49 [165] (shown in Figure 1.10).

One of the main approaches to study strangeness production are transport models [106, $168,109,98,164]$, especially to establish a baseline calculation based on vacuum resonance properties. Additional medium effects like the onset of a QGP phase can then be identified by finding discrepancies of such approaches to experimental data. One option to isolate inmedium effects is to first constrain the production cross-section (or mechanism in general) in elementary collisions. The influence of the surrounding medium can then be tested by comparing it with experimental data for larger systems. At lower beam energies, where no QGP is expected, many open questions remain regarding medium effects, including the nature of kaon-nucleon potentials or medium-modified cross-sections [169].

Apart from transport approaches, thermal models are employed, for example, in [170]. The agreement with data for light and single strange hadrons to experimental data is surprisingly good for different energies [171]. However, at lower beam energies, differences are seen for rare double strange hadrons $[68,172]$. Since thermal models assume a complete chemical equilibrium at a relatively early time in the collision, finding discrepancies in the experimental data always hints at interesting additional non-equilibrium effects. 


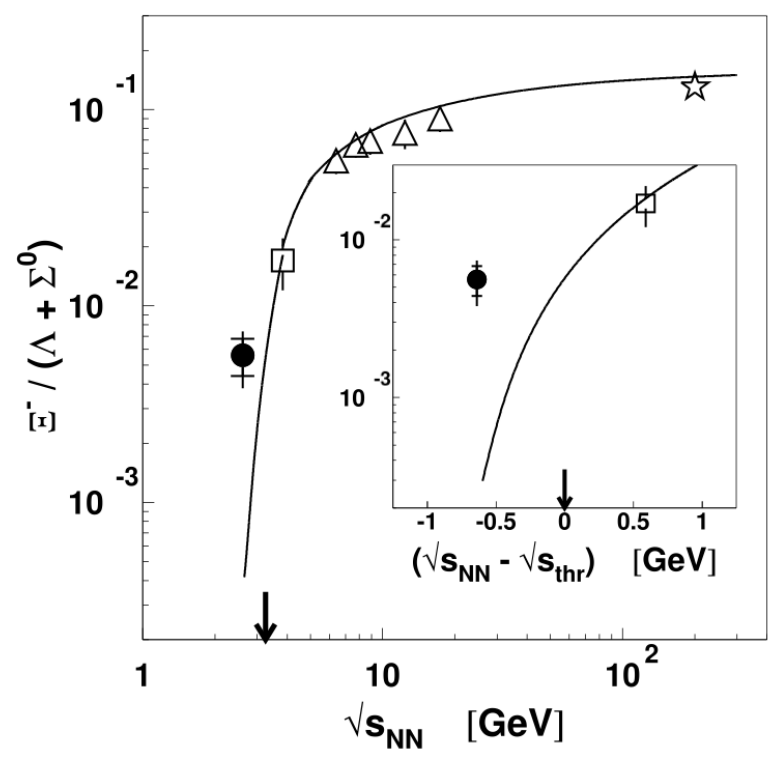

Figure 1.11: Comparison of the $\Xi^{-} /\left(\Lambda+\Sigma^{0}\right)$ ratio for different beam energies. Full line thermal model prediction. Result from [68].

In this work, (Section 3.2) the low beam energy kinematic regime reached by the SIS18 accelerator, which is close to the production threshold [173], is studied. The studies are partly motivated by the mentioned discrepancies that appeared in the comparison of thermal model calculations to data (as seen in Figure 1.11 for the $\Xi$ ). The $\Xi$ baryon and the $\phi$ meson are enhanced $[68,174]$. There are several attempts to explain these high production yields $[175,176,177]$. The focus in this work is on the possible explanation from [177], which resolves the discrepancy between theory and experiment by introducing a new strangeness production mechanism based on the decay of baryonic resonances in a hadronic transport approach. Before the new mechanism is added and consequences are discussed, the current status of strangeness production is introduced at the beginning of Section 3.2.

\subsection{3 "Snowballs in hell" - deuterons in heavy-ion reactions}

As heavy nuclei collide in accelerator experiments, several species of light nuclei are formed in the aftermath and measured by the experimental detectors [178, 179]. Light nuclei include d, t $,{ }^{3} \mathrm{He},{ }^{4} \mathrm{He}$, which are compound states of nucleons, and hyper-nuclei like ${ }_{\Lambda}^{3} \mathrm{H}$, which include a hyperon instead of a nucleon. The focus here is the production of deuterons $(d)$, which is a relatively weakly bound state of $p$ and $n$.

Light nuclei are of research interest since it is unclear if their measurement in cosmic rays is due to ordinary scatterings of protons on other nuclei or if they hint at beyond-standard model physics [180]. Furthermore, in collider experiments they might indicate nucleon density fluctuations, which in turn are connected to the critical point [181]. Accounting for the production of $d$ and the simultaneous loss of nucleons is also important when calculating and predicting nucleon yields [182].

How and when in a nucleus-nucleus collision light nuclei are produced is an open question $[180,183]$ since the different model assumptions about their formation are contrary. Three 

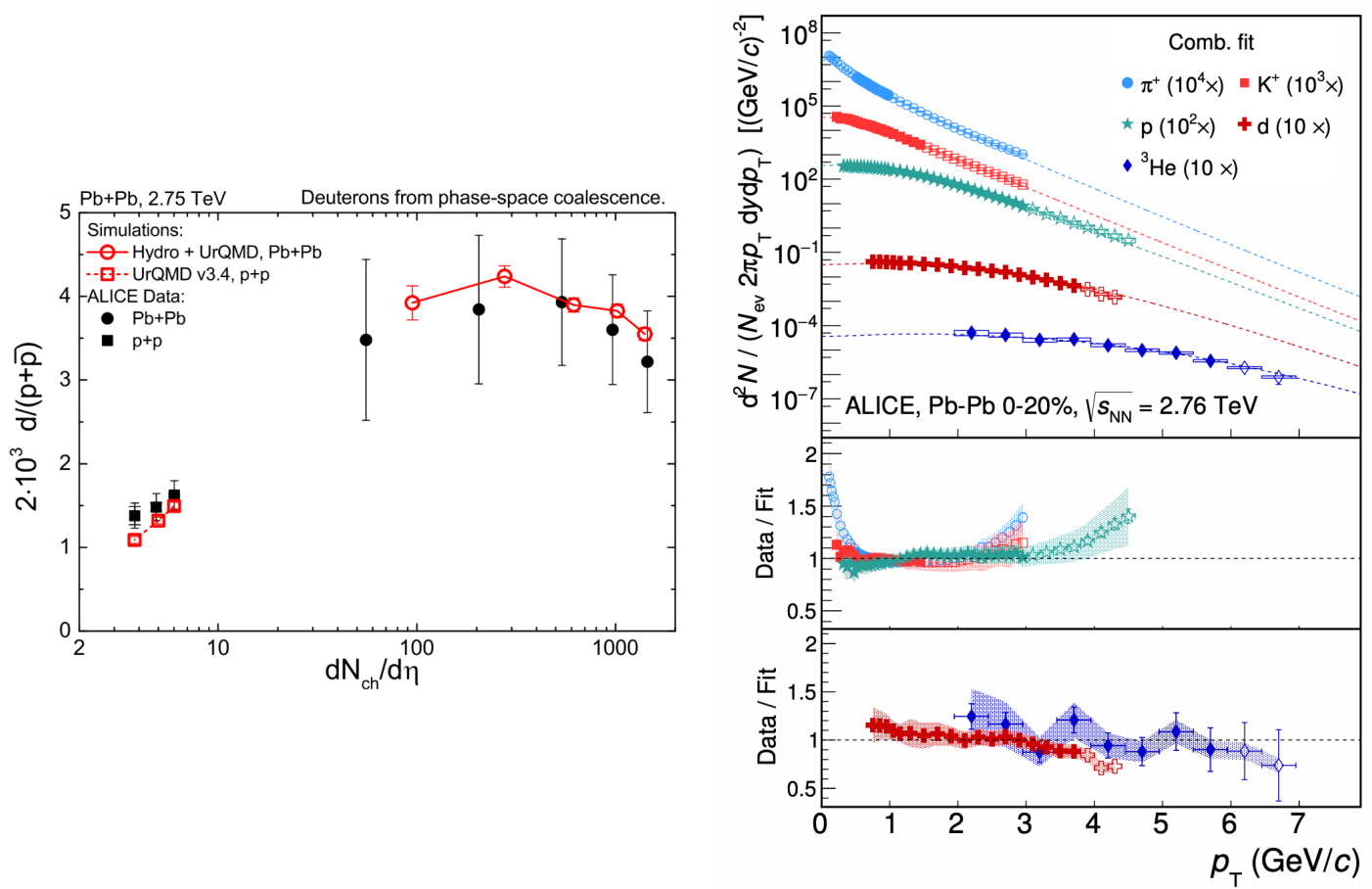

Figure 1.12: Different approaches for deuteron production in $\mathrm{PbPb}$ collisions at $\sqrt{s_{N N}}=$ $2.76 \mathrm{TeV}$ match the experimental data. Left: Deuterons from phase-space coalescence. Result of $d /(p+\bar{p})$ ratio from [182]. Right: Deuterons from thermal blast-wave model (dashed line). Result of transverse momentum spectra from [69].

different approaches are pursued to describe the $d$ production (see [180] for more details and model references). Coalescence models assume deuterons to be formed at the final stages by nucleons close in phase space. Such models differ if they take the nucleon wave function into account and if they model the particle evolution dynamically (via transport and hydrodynamical approaches). Coalescence models are able to match the measured $d$ production (e.g. [182], left plot in Figure 1.12). In contrast to the assumption of late production, are thermal model calculations that are paired with a blast-wave model. They expect an earlier deuteron production due to the assumed early chemical freeze-out and are also able to match the experimental data (right plot in Figure 1.12). Since the freeze-out temperature is assumed to be close to the critical temperature $T_{C}$, it is unclear how deuterons with a binding energy of a couple $\mathrm{MeV}$ could survive temperatures of more than a hundred $\mathrm{MeV}$. This paradox is coined as $d$ being like "snowballs in hell". The third approach are dynamical approaches that microscopically form and destroy deuterons. They can follow the $d$ evolution over time and investigate whether the "snowballs" survive the "hell".

In this work, a dynamical approach is chosen to study the deuteron production on the example of AuAu collisions at 7.7 GeV (Section 5.3). Since the most important reactions involving $d$ are multi-particle catalysis reactions $(\pi p n \leftrightarrow \pi d$ and $N p n \leftrightarrow N d,[135,184])$, the new treatment of multi-particle reactions in a hadronic transport approach (Section 4.3.2) allows to investigate the deuteron production in the late stages and the "snowballs in hell"-paradox microscopically. Studying the deuteron production microscopically is an idea already explored in the literature in $[126,185,186,127]$. These earlier works mainly 
differ from the presented approach in the set of $d$ reactions that are included. The differences originate in part from the difficulty of accounting for multi-particle reactions. Most similar is the approach in [126], which also treated the nucleon 3-to-2 catalysis reaction in a stochastic manner but neglects the $\pi p n \leftrightarrow \pi d$ reaction due to only studying lower beam energy collisions. A recent extension of this approach is found in [127] focusing on the effect of the finite $d$ size in small collision systems.

\subsubsection{The "proton anomaly"}

Thermal models are very successful in reproducing the yield of especially light hadrons over a large range of energies with a few parameters. An interesting exception is the proton yield at LHC energies [187, 188, 189, 190], which is overpredicted. This exceptional

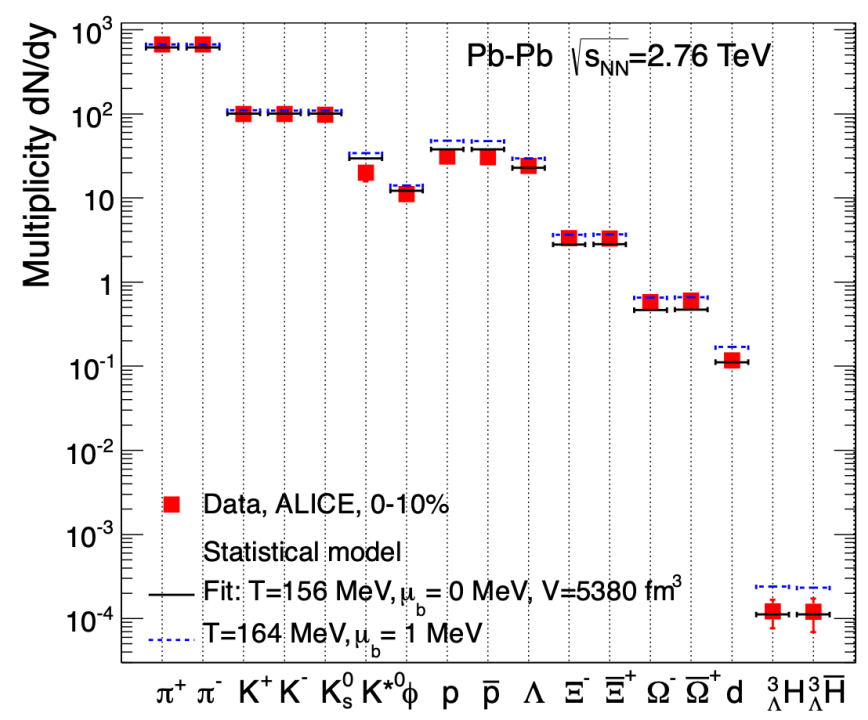

Figure 1.13: Comparison of the thermal model fit to experimental data from ALICE. Note that $p$ shows the largest deviation to the fit, when normalized to the experimental error [190]. Result from [190].

discrepancy (seen in Figure 1.13) is referred to as the proton anomaly.

The anomalous proton yield sparks interest since it is related to ongoing debates about the late out-of-equilibrium stages in heavy-ion collisions. The underlying assumption of thermal models is an early chemical freeze-out without any late rescattering. On the other hand, Microscopic transport models find inelastic scatterings until much later times and thereby a later chemical freeze-out. Late-stage rescatterings are found to be significant for final hadron spectra $[66,67]$. Sizable changes to the spectra are important since they are used to constrain the QCD transport coefficients [78, 191]. The proton yield is also specifically sensitive to the switching temperature [133], an important parameter in hybrid approaches.

The proton overprediction is one of the main signatures of non-equilibrium rescatterings since it is attributed to the (in thermal models) missing baryonic annihilation reactions. Indeed, microscopic (transport) calculations find an improved agreement with the experimental data, if annihilations are included [66, 67, 192, 193]. Note that there are also extensions of thermal model calculations with $\pi$ nucleon interactions that explain 
the anomaly [189]. A missing piece of the different microscopic models, that attribute the anomaly to annihilations, is the back-reaction of the annihilation reaction. Baryonantibaryon annihilations produce multiple pions, but multi-particle reactions are, as argued in Section 1.2.6, challenging to account for in transport approaches. Therefore, detailed balance is explicitly broken for the annihilation reaction (an exception is found in $[129,130])$. Non-microscopic calculations estimate the regeneration of $B \bar{B}$ pairs due to the back-reaction to be from $20 \%$ [194] up to a full regeneration with no remaining baryon depletion [131]. A first study taking the back-reaction into account microscopically in multiple steps even found a small net-enhancement for protons at LHC energies [130].

In this work, the multi-particle $5 \pi$ back-reaction for the $p \bar{p}$ annihilation is accounted for in a microscopic transport approach for the first time. This allows to quantify the regeneration of (anti-) protons in the late non-equilibrium stages of collisions (Section 5.4). The longstanding question whether the annihilation reactions are relevant for the final $p$ yield and whether they potentially alleviate the $p$ anomaly is thereby addressed.

\subsection{Structure of this thesis}

The various current research topics that are motivated above are addressed in this thesis by employing a hadronic transport approach to calculate observables of the non-equilibrium stages in low, intermediate and high energy heavy-ion reactions. The overarching question is what the reaction dynamics and particle properties within the hadron gas are. The employed transport model chosen for this purpose is detailed in the following Chapter 2. This thesis' main contribution is the extension of the approach for binary interactions to also account for stochastic multi-particle interactions. The presented results are therefore categorized by the microscopic interactions that are included in the calculations.

First, the evolution of the hadron gas phases in heavy-ion collision with the existing binary reactions is probed and constrained in detail by two complementary observables in Chapter 3: dilepton and strangeness production. Dilepton emission (Section 3.1) in particular allows to understand the existing dynamic since the electromagnetic probe does not alter the strongly-interacting dynamic. Elementary and intermediate-size systems are studied and compared to a large set of experimental data to verify and constrain the vacuum and binary cascade. The larger systems are studied to identify and predict potential in-medium effects by also employing a coarse-graining approach. Similarly, also for the strangeness production (Section 3.2), differently sized systems are studied to identify medium effects. Particular emphasis is placed on an additional production mechanism for double strange hadrons.

The established cascade baseline of the binary evolution is used as the basis to introduce multi-particle reactions to the approach in Chapter 4. Multi-particle reactions require a different stochastic collision treatment. The necessary theoretical background and derivations for 3-body and 5-body reactions are pedagogically presented (Section 4.1). Afterwards, the extension of the transport model with the stochastic collision criterion (Section 4.2) and multi-particle reactions (Section 4.3) is described. The results with multi-particle reactions are discussed in Chapter 5. First, the new stochastic collision treatment is extensively verified (Section 5.1) by comparisons to analytic expectations and an investigation of its numerical stability. Then, the effect of Dalitz decay backreaction is studied (Section 5.2) and topical research questions related to the production of light nuclei (Section 5.3) as well as protons (Section 5.4) in the late collision stages are addressed. The latter signify the main results of this thesis. 
Both avenues of study, the investigation of dilepton or strangeness production and of multi-particle reactions, are ultimately motivated by the study of high-density collisions: dilepton and strangeness production allow to probe the in-medium effects. Dileptons are sensitive to the vector meson spectral function inside the medium and strangeness production to potentials, in-medium cross-section and sub-threshold production mechanisms. The addition of multi-particle reaction intervenes in the microscopic dynamic itself, which could be seen as an additional medium effect and extends the applicability of transport approaches to higher densities.

The thesis is closed with conclusions and an outlook in Chapter 6 . 



\section{Chapter 2}

\section{The hadronic transport approach SMASH}

The calculations in this thesis are obtained by employing the hadronic transport approach SMASH (Simulating Many Strongly-interacting Hadrons) [98]. SMASH, like all transport approaches, is based on the Boltzmann equation (Eq. 1.8). The aim is to calculate an effective solution for the particle density function $f$ over time (Section 2.2). The approach is suitable to calculate the dynamics of a gas of hadrons as created in heavy-ion collisions. Because transport models are successfully employed over the last decades, a major goal of the more recent SMASH approach is to combine the successful aspects and experiences of previous approaches [99, 100, 101, 102, 103, 106, 107, 108, 109, 110, 111, 112].

The approach was first introduced in [98] and is already successfully employed for a large range of topics: Studies were conducted on the production of different particles (deuterons [135, 184], strange hadrons [2, 195], photons [196], resonance production at LHC energies [197]), on different aspects and observables of the hadronic medium (forced canonical thermalization [125], equilibration and freeze-out when employing an expanding metric [198], influence of the neutron-skin effect [199], baryon stopping in the context of string fragmentation [200], jet quenching in the hadron gas [201], collective flow at SIS energies [202], critical behavior with a density functional equation of state [203]) and on the transport coefficients that result from the employed degrees of freedom and interactions (shear [204] and bulk [205] viscosity, electrical conductivity [206] and crossconductivity [207]). Furthermore, SMASH is employed in the JETSCAPE hybrid studies [78] and was part of different systematic transport approach comparisons [113, 208, 97].

In this chapter, the different relevant aspects of the approach are introduced. It is based on the original introduction in [85] and [1] with more recent developments being mentioned as well. The extension of the approach with multi-particle reactions and the new stochastic collision criterion are given in Section 4.2 and 4.3 after presenting the necessary derivations in Section 4.1.

SMASH is freely available as an open-source $\mathrm{C}++$ code $[209,210]$ to ensure transparency and reproducibility of all results ${ }^{1}$. The used version is mentioned in the specific result sections. More details on technical aspects of the approach are found in Appendix A.

\footnotetext{
${ }^{1}$ Employed in this thesis are the versions SMASH-1.1, SMASH-1.6 [211], SMASH-1.7 [212], SMASH-2.0.1 [213] or the upcoming SMASH-2.1 version.
} 


\subsection{Degrees of freedom}

The degrees of freedom of the approach are hadrons ${ }^{2}$. Most hadronic degrees of freedom listed by the Particle Data Group (PDG) [56] up to a mass of $2.35 \mathrm{GeV}$ are included, which in particular contains all hadrons with a 3- or $4-^{n * "}$ experimental data rating by the PDG. The included mesons are pseudo-scalar states like $\pi, \eta, \eta^{\prime}$, vector states like $\rho, \omega, \phi$, scalar states like $\sigma, f_{0}, a_{0}$, strange mesons like $K$ and higher mesonic resonances. Included baryons are $N, \Delta, \Lambda, \Sigma, \Xi$ and $\Omega$, as well as the excited baryonic resonance states $\left(N^{*}, \Delta^{*}, \ldots\right)$. A full list is found in [98] with an update focused on the strange sector in $[2]^{3}$.

Particle properties are generally taken from the PDG Book [56] and include mass, decay width and parity. They are varied within the experimental limits to optimally reproduced available experimental observables. Small mass differences for isospin partners are neglected since cross-sections are isospin symmetric in general. The only exception are certain $N N$ cross-sections as e.g. the isospin asymmetric $\eta$ production introduced in Section 3.1.1. Particles with a decay width smaller than $10 \mathrm{keV}$ (e.g. $\pi, \eta, K, \ldots)$ are considered to be stable i.e. they do not decay hadronically ${ }^{4}$.

\subsubsection{Resonances}

All particles with a decay width larger than $10 \mathrm{keV}$ are considered to be unstable resonances. Resonances have vacuum properties and are tuned to reproduce the elementary cross-sections. All resonances have Breit-Wigner-shaped spectral functions, which include a mass-dependent width, which follows the idea from Manley and Saleski in [214]. The resonance properties are, however, updated in comparison to [214] with current PDG values. Note that assuming a Breit-Wigner spectral function for $\sigma$ is known to be questionable. The approximation is made, and the resonance properties are tuned to model the quasi-elastic scattering of $\pi \pi$.

\section{Spectral functions}

In general, SMASH only employs vacuum spectral functions. They remain constant throughout the system evolution, since they are assumed to be independent from temperature or density. This provides a baseline to probe additional in-medium modifications. All resonance spectral functions are relativistic Breit-Wigner functions:

$$
\mathcal{A}(m)=\frac{2 \mathcal{N}}{\pi} \frac{m^{2} \Gamma(m)}{\left(m^{2}-M_{0}^{2}\right)^{2}+{ }^{2} \Gamma(m)^{2}} .
$$

Here $M_{0}$ is the pole mass and $m$ is the actual mass of the resonance. The normalization factor $\mathcal{N}$ is chosen such that

$$
\int_{0}^{\infty} d m \mathcal{A}(m)=1 .
$$

The normalization factor can deviate from 1 since the decay width is mass-dependent. The deviation is below $55 \%$ for all particles, with most normalization factors close to unity. Energy-momentum conservation is always enforced in the propagation and creation of resonances. In this sense, all resonances are always on the mass shell (on-shell). The

\footnotetext{
${ }^{2}$ except for the later-described (di)leptons, photons and deuterons

${ }^{3}$ The up-to-date degrees of freedom and their properties of the different SMASH version are always accessible through the particles.txt and decaymodes.txt files [209].

${ }^{4}$ They are still considered for the electromagnetic decays into photons and dileptons, see Section 2.4.
} 


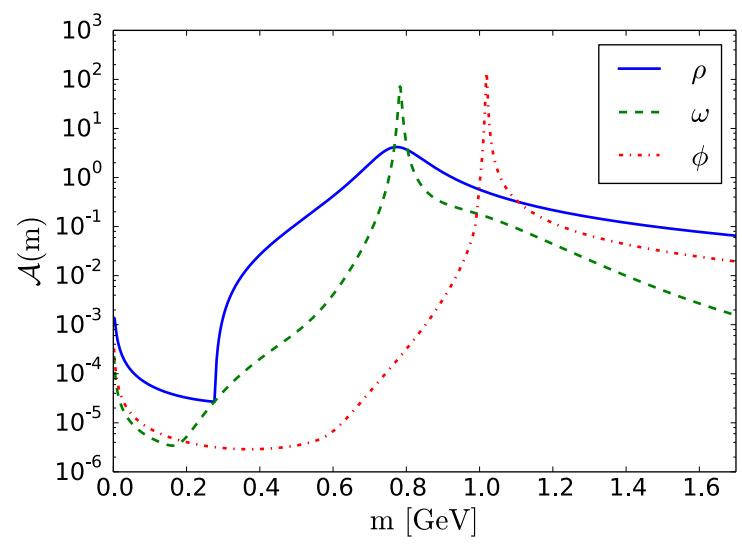

Figure 2.1: Spectral Function of the vector mesons with dielectron decay mode.

resonance mass is fixed throughout the resonance lifetime and sampled upon formation according to the spectral function and the available phase space.

The spectral functions vanish at the combined mass of the lightest decay products. It is important to note that the dilepton decay modes (discussed in Section 2.4) are correctly taken into account as the kinematic threshold. The spectral functions of resonances that directly decay into a lepton pair $\left(R \rightarrow l^{+} l^{-}\right)$have contributions below the lightest combined mass of hadronic decay products, which is referred to as the hadronic threshold here. In Figure 2.1 the spectral functions of the three vector mesons with dielectron decay channels are depicted as an example. The spectral functions peak at the pole mass $M_{0}$ and, as expected from their widths, the $\rho$ peak is the broadest, followed by the $\omega$ and the sharp $\phi$. Noticeable is the kink for the $\rho$ spectral function at around $0.3 \mathrm{GeV}$. The decay with the lightest hadronic decay products for the $\rho$ is $\rho \rightarrow \pi^{+} \pi^{-}$, so the hadronic threshold is at $2 m_{\pi}$. This threshold leads to the kink because the partial hadronic decay width of the $\pi$ decay vanishes at this mass. Since the $\rho$ can also decay into an $e^{+} e^{-}$ pair the spectral function continues down to $2 m_{e}$. This is also true for the $\omega$ and the $\phi$. Both spectral functions also have contributions down to the combined mass of the actual lightest decay products - the dielectron mass. This treatment of the leptonic thresholds was introduced in [85] for the $\rho$ meson and is extended to all three vector mesons in this work. Other approaches $[168,163]$ neglect the contributions below the hadronic threshold for numerical reasons (see also footnote in Section 2.4.1). The sub-threshold contributions are of particular interest for the dilepton emission through $R \rightarrow l^{+} l^{-}$, since it allows to quantify their effect on the total dilepton spectrum in the low-mass region (see also the discussions in Section 3.1.2) and compare to thermal dilepton emission in coarse-graining approaches over the whole invariant mass range. Note, however, that in a Monte-Carlo approach, it is challenging to numerically populate this mass region below the hadronic threshold due to the small spectral function (Figure 2.1), which leads to visible statistical fluctuations in some of the dielectron invariant mass spectra presented in Section 3.1.

\section{Decay widths}

The non-trivial shape of the spectral function, as e.g. seen in Figure 2.1, originates from the mass dependent decay width $\Gamma(m)$. The lifetime of the resonances is given as $\tau=1 / \Gamma(m)$. The width is the sum of all partial decay widths for the different decay modes.

$$
\Gamma(m)=\sum_{i} \Gamma_{i}(m)
$$


An example of the results of this summation and the following treatment for particle decay widths is shown in Figure 2.2 for the $N^{*}(1440)$ resonance.

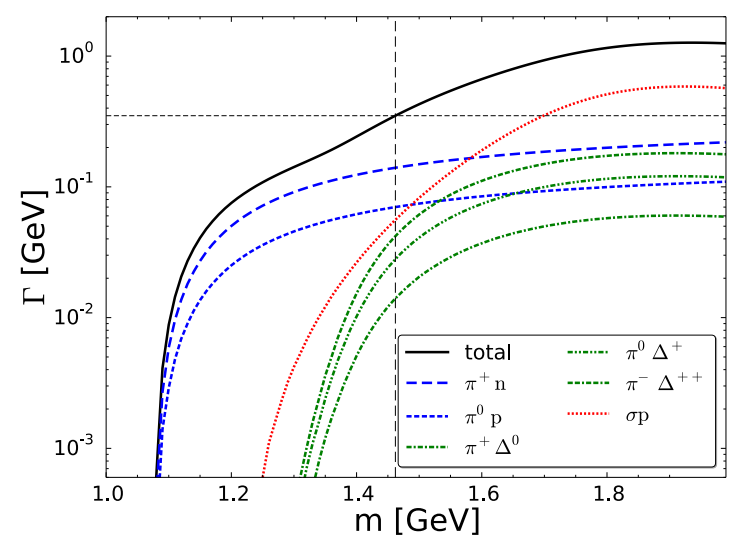

Figure 2.2: Total and partial decay widths of the $N^{*}(1440)$ resonance. Figure from [98].

All hadronic partial widths are calculated following the framework of Manley et al. [214] (without taking the same parameters for the resonance properties). The partial width of a two-body resonance decay $R \rightarrow a b$ is calculated as follows

$$
\Gamma_{R \rightarrow a b}=\Gamma_{R \rightarrow a b}^{0} \frac{\rho_{a b}(m)}{\rho_{a b}\left(M_{0}\right)}
$$

with the resonance mass $m$, the resonance's pole mass $M_{0}$ and $\Gamma^{0}=\Gamma\left(M_{0}\right)$. The function $\rho_{a b}(m)$ is defined as

$$
\begin{aligned}
\rho_{a b}(m)= & \int d m_{a} d m_{b} \mathcal{A}_{a}\left(m_{a}\right) \mathcal{A}_{b}\left(m_{b}\right) \\
& \times \frac{\left|\vec{p}_{f}\right|}{m} B_{L}^{2}\left(\left|\vec{p}_{f}\right| R\right) \mathcal{F}_{a b}^{2}(m),
\end{aligned}
$$

where $m_{a}$ and $m_{b}$ are the masses of the decay products $a$ and $b, \mathcal{A}_{a / b}$ is their respective spectral function and $\left|\vec{p}_{f}\right|$ the absolute value of the final-state momentum of $a$ and $b$ in the center-of-momentum frame. Equation 2.5 also includes the "Blatt-Weisskopf functions" $B_{L}[215]$ and the form factor $\mathcal{F}_{a b}[216]$.

Note that this resonance treatment excludes any decays for which the combined pole mass of the final state particles is larger than the pole mass from the decaying resonance, although such a decay would be strictly physical speaking possible. The reason is that the absolute final state momentum is undefined in this case, as its square is defined as follows

$$
\begin{aligned}
\vec{p}_{f}^{2} & =\vec{p}_{\mathrm{cm}}^{2}\left(m, m_{a}, m_{b}\right) \\
& =\frac{\left(m^{2}-\left(m_{a}+m_{b}\right)^{2}\right)\left(m^{2}-\left(m_{a}-m_{b}\right)^{2}\right)}{4 m^{2}},
\end{aligned}
$$

which for $m=M_{0}$ (note the denominator in Eq. 2.4) is negative.

The parameterizations employed for the dilepton decays are described in Section 2.4.1. The decay width prescription above is not applied to dilepton decays. 


\subsubsection{Deuterons}

Deuterons in SMASH $[183,184]$ are treated like on-shell point-particles as in $[126,185,186]$. The applicability of this approach specifically for the here studied late stages of a heavyion collision depends on the time in the evolution: the deuteron mean free path at the start of an afterburner evolution is comparable to twice the geometric size of deuteron wave-function. In contrast, at later stages, the mean free path increases, making the approach more justifiable. Because of this, "deuterons" are better thought of as correlated nucleon pairs. However, it seems that the inclusion of the finite deuteron size is not important in heavy nucleus-nucleus collisions - a recent study [127] on the inclusion of the finite deuteron size in a similar approach only found a significant effect for a much smaller fireball created in $p p$ collisions, which are not discussed in this work. As shown in $[183,184]$, the approach with on-shell point-like $d$ describes the data for heavy-ion collisions reasonably well and can capture the fact that deuterons (or "correlated nucleon pairs") are created and destroyed. Deuterons are the only light nuclei included in SMASH, even though their treatment could be extended to include other light nuclei in the future.

Deuterons are produced or destroyed through catalysis by pions or nucleons in the following reactions and their CPT-conjugates: $\pi d \leftrightarrow \pi n p, N d \leftrightarrow N n p, \bar{N} d \leftrightarrow \bar{N} n p$ and $\pi d \leftrightarrow$ $N N$. Additionally, elastic reactions for $\pi d, N d$ and $\bar{N} d$ are included. The challenge in a transport approach is to treat the 3-body catalysis reactions microscopically. For the usual applied geometric collision criterion, no generalization for multi-particle reactions is available. This situation is resolved in this work by introducing the new stochastic collision criterion or by a chain of binary reactions (see Section 4.3.2).

\subsubsection{Test particle method}

In BUU-type approaches like SMASH, the spacial particle distribution function is represented by many point-like test particles ${ }^{5}$. The number of particles is scaled with the test-particle number $N_{\text {test }}$ as

$$
N \mapsto N N_{\text {test }} .
$$

In order to not change the intrinsic dynamics, e.g. the scattering rate, the likelihood of binary scatterings has to be scaled down by mapping the cross-section as

$$
\sigma \mapsto \sigma N_{\text {test }}^{-1} \text {. }
$$

For multi-particle scatterings, the required mapping for the collision probability is discussed in Section 4.1.6. Reducing the cross-section in this way also decreases the issues of non-locality of interactions (see Section 2.2.2), since the finite distance over which particles interact is reduced. Higher numbers of test particles also increase the accuracy of the phase space density, which is required for the calculation with potentials (Section 2.2.1).

\subsection{Effective solution of the Boltzmann equation}

All transport approaches aim to provide an effective solution of the Boltzmann equation ${ }^{6}$ :

$$
\frac{\partial f}{\partial t}+\frac{\vec{p}}{m} \nabla f+\vec{F} \frac{\partial f}{\partial \vec{p}}=\left(\frac{\partial f}{\partial t}\right)_{\text {coll }} .
$$

\footnotetext{
${ }^{5}$ In contrast to QMD approaches that use Gaussian wave packets. Only for the extraction of thermodynamic quantities are the particles in SMASH are assumed to be non-point-like and distributed in space.

${ }^{6}$ Note that here the non-relativistic formulation is used, but a covariant formulation is also possible.
} 
This is achieved by accounting for the different terms of the equation on a microscopic level since a proper analytic solution is generally not attainable. The left side of the equation describes the time evolution of the particle distribution function in the absence of collisions ${ }^{7}$. The second term on the left side is known as the free streaming term, which accounts for particles flying along straight lines according to their momenta. The third term describes the effect of an external force field on the particle trajectories. Finally, the right-hand side, the collision term, accounts for the interaction of the particles. This section is split into the discussion of the left side of the equation in Section 2.2.1 and its right side in Section 2.2.2 and Section 2.2.3.

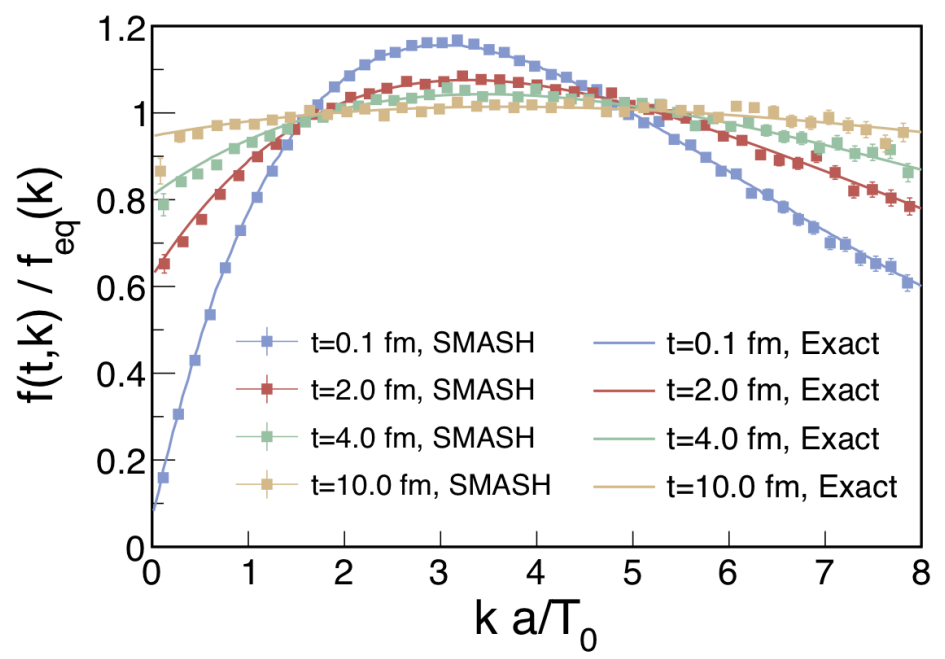

Figure 2.3: Ratio of the distribution function over the equilibrium Boltzmann distribution for different times. Figure from [198].

As mentioned, in general, it is not possible to solve the equation analytically. Therefore, it cannot be verified if a transport approach provides an effective solution to the Boltzmann equation. However, an exact solution can be obtained in a Friedmann-Robertson-Walker spacetime with an expanding metric, which can also be implemented in transport approaches [198]. Figure 2.3 shows the comparison of the exact solution with the one obtained by SMASH in the case of an expanding metric. The result shows that SMASH effectively provides a valid solution of the Boltzmann equation in this setup.

\subsubsection{Propagation}

Hadrons are generally propagated along straight lines according to their momenta in accordance with the free-streaming term of the Boltzmann equation.

The propagation of the evolution in transport approaches is often numerically separated into timesteps. In SMASH, the role of timesteps has changed since its original introduction [98]. The system is now propagated ${ }^{8}$ from interaction to interaction (similar to e.g UrQMD [106]), which has the major advantage of no longer needing the assumption that each particle only interacts once during one timestep. Note that the latter introduced stochastic collision criterion requires the revision of this advancement. Since the collision probability is only defined over an a priori known time-interval $\Delta t$, the propagation is

\footnotetext{
${ }^{7}$ Without the collision term, the equation is referred to as the Vlasov equation.

${ }^{8}$ Note that this propagation is technically still done within a given timestep, at which certain quantities are evaluated, for example, the potentials.
} 
done over the whole timestep at once. The assumption that each particle only interacts once during one timestep is therefore reinstated for the stochastic collision criterion (see discussions in Section 5.1.1 and 5.1.5).

To account for attractive and repulsive forces between particles, mean-field potentials are employed in SMASH (third term on the left of the Boltzmann Equation). An effective nucleon-nucleon interaction is mediated by a Skyrme potential of the form

$$
U\left(\rho, \rho_{I 3}\right)=a\left(\rho / \rho_{0}\right)+b\left(\rho / \rho_{0}\right)^{\tau} \pm 2 S_{\mathrm{pot}} \frac{\rho_{I 3}}{\rho_{0}},
$$

where $\rho$ is the Eckart rest frame baryon density, $\rho_{I 3}$ the Eckart rest frame density of the relative isospin projection $I_{3} / I$ and $\rho_{0}=0.1681 / \mathrm{fm}^{3}$ is the nuclear ground state density. The default parameters $\left(a, b, \tau, S_{\text {pot }}\right)$ are taken from a transport code comparison [217]. Only baryons are affected by the potential. The potentials lead to curved trajectories ${ }^{9}$ according to the following modified equation of motions, which are given in their relativistic form here.

$$
\begin{aligned}
\frac{d x_{i}^{\mu}}{d t^{*}} & =u^{\mu}-\frac{\partial H^{*}}{\partial p_{i}^{*}} \Lambda_{i}^{\mu} \\
\frac{d p_{\mu}}{d t^{*}} & =\frac{\partial H^{*}}{\partial x_{i}^{\mu}}
\end{aligned}
$$

Quantities labeled with a ' $*$ ' are meant to be evaluated in the local rest frame. The boost $\Lambda$ is from an arbitrary frame into the local rest frame $\Lambda: x \rightarrow x^{*}$. $u$ is the flow 4-velocity in the computational frame. For the Skyrme interaction, the Hamiltonian for particles $i$ is given by

$$
H_{i}^{*}=\sqrt{-p_{j}^{*} p^{j *}+m^{2}}+U(\rho)
$$

In addition, a generalization of the Skyrme potential is explored with SMASH [203], which employs vector-density-dependent interactions (in contrast to the rest-frame-density dependence above). The extension allows realizing equations of state for dense nuclear matter that include phase transitions and a critical point and to study their effects on the hadronic evolution. In the future, the approach for potentials will be further extended to also include a scalar-density-dependence of the potentials as they exist in the Walecka model $[218,219]$ and to mediate an electromagnetic interaction between charged particles (similar to $[220,109])$.

All results in this thesis are cascade calculations i.e. potentials are not used.

\subsubsection{Geometric collision criterion}

At the core of microscopic (transport) approaches like SMASH, where point-like (test) particles propagate and interact, the decision if and when they collide has to be made. The collision criterion is important for this thesis since it needs to be extended to include multiparticle reactions. In this section, the common geometric criterion is described. The new (stochastic) criterion is discussed in Section 4.2 after presenting its theoretical background in Section 4.1. As the cross-section governs the likelihood of a binary interaction, the

\footnotetext{
${ }^{9}$ Numerically, particles are still propagated on straight lines within a timestep. Their momenta are updated according to the equation of motion at the timestep interval.
} 
main input to the criterion is the total cross-section, which contributions are detailed in the following section.

The geometric approach to decide when two particles scatter is based on a geometric interpretation of the total cross-section [99]. If a collision is accepted by the criterion and there are different channels contributing to the total cross-section, a channel is chosen randomly with the weights $\sigma_{\text {partial }} / \sigma_{\text {total }}$. For a collision to be accepted, the transverse distance of closest approach $d_{T}$ has to smaller than the interaction distance $d_{\text {int }}$ given by the cross-section:

$$
d_{T}<d_{\text {int }}=\sqrt{\frac{\sigma}{\pi}}
$$

There are several definitions available for the transverse distance of closest approach $d_{T}$ of two particles [106, 221, 222]. SMASH followed the approach introduced by UrQMD [106] up to version SMASH-2.0 [213], which defines

$$
d_{T}^{2}=\left(\overrightarrow{r_{a}}-\overrightarrow{r_{b}}\right)^{2}-\frac{\left(\left(\overrightarrow{r_{a}}-\overrightarrow{r_{b}}\right) \cdot\left(\overrightarrow{p_{a}}-\overrightarrow{p_{b}}\right)\right)^{2}}{\left(\overrightarrow{p_{a}}-\overrightarrow{p_{b}}\right)^{2}}
$$

with the particles $a$ and $b$ position $\vec{r}$ and momentum $\vec{p}$. The time of the collision is given as the time of closest approach calculated by

$$
t_{\mathrm{coll}}=-\frac{\left(\overrightarrow{r_{a}}-\overrightarrow{r_{b}}\right) \cdot\left(\overrightarrow{p_{a}} / E_{a}-\overrightarrow{p_{b}} / E_{b}\right)}{\left(\overrightarrow{p_{a}} / E_{a}-\overrightarrow{p_{b}} / E_{b}\right)^{2}}
$$

The main issue with this formulation is that the time ordering is not unique since the formulation is not covariant [221]. The result is a frame dependence of the evolution since the time ordering decides which particles interact first if multiple scatterings are possible (i.e. accepted by the criterion).

In the recent version SMASH-2.0 [213], the approach for criterion is therefore changed to the fully covariant formulation ${ }^{10}$ given in [222], which is employed in the transport approach JAM [110] and builds on the idea of Kodama [221].

Note that even though the time ordering is frame-independent in this formulation. All criteria (including the new stochastic one) are non-local in the sense that they encode instantaneous interaction over a finite distance ${ }^{11}$, which gives rise to causality violations [224]. The non-locality is mainly alleviated by the use of test particles to reduce the reaction range.

The main disadvantage of the geometric criterion is the lack of a successful generalization of $d_{T}$ to more than two particles. This deficiency can either be alleviated by employing the stochastic criterion or, in certain cases, by employing a chain of binary reactions (see Section 4.3 for examples). Note that the latter is only possible if physical intermediate reactions are known ${ }^{12}$ and therefore less flexible than employing the stochastic criterion. The stochastic criterion also is inherently covariant.

\footnotetext{
${ }^{10}$ Since the covariant equations require a more extensive notation, the reader is directly referred to the presentation in Section 5.1 of [222] or [223].

${ }^{11}$ Strictly speaking the non-locality is not caused by the criteria, but by the instantaneous treatment of interactions in the transport approach in general.

${ }^{12}$ Otherwise, artificial steps have to be introduced, see Section 4.3.2.
} 


\subsubsection{Interactions}

The transport approach maps the different parts of the collision term (see also Section 4.1.2) to different types of interactions. The 5 main categories are binary elastic and inelastic scatterings, the formation and decay of excited resonances, and string fragmentation. A sixth category is added in this thesis: multi-particle reactions, which are introduced in Section 4.3). The emission of electromagnetic probes (dileptons and photons) is not counted as a category since they are treated perturbatively (Section 2.4).

As explained above, the primary input for the collision criterion is the total cross-section $\sigma_{\text {tot }}$ of two particles, which is defined as

$$
\sigma_{\text {tot }}=\sum \sigma_{\text {partial }} .
$$

$\sigma_{\text {partial }}$ are the partial cross-sections of different possible interactions between the incoming particles of the collision. The definitions of the different binary interactions and their partial cross-sections are discussed below. To illustrate that these contributions to the total
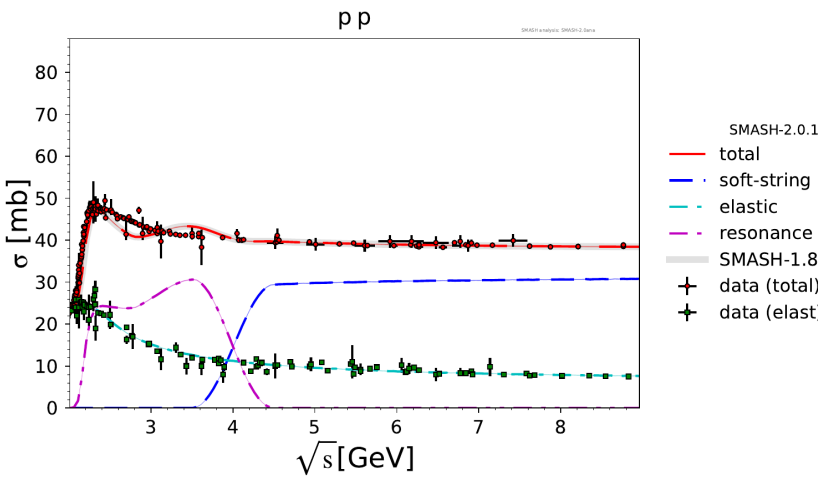

- soft-string

- - elastic

- resonance

- SMASH-1.8 (total)

$+\quad$ data (total)

+ data (elast)

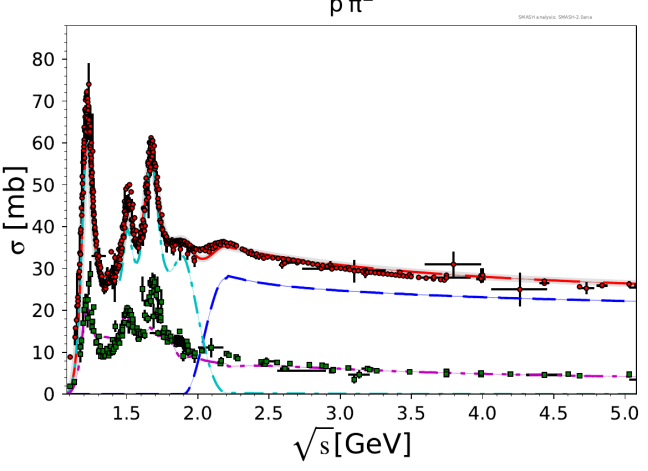

SMASH-2.0.1

- total

- soft-string

- resonance

- - elastic

SMASH-1.8 (total)

+ data (total)

+ data (elast)

$\pi^{+} \pi^{-}$

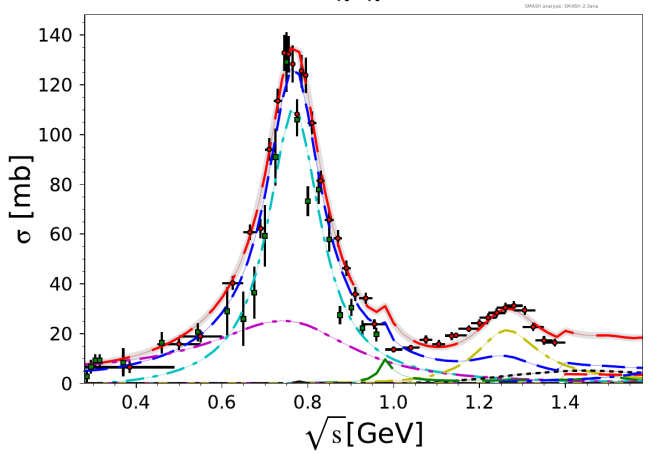

SMASH-2.0.1

— total

- $\rho$

$\begin{array}{ll}- & \sigma \\ - & - \\ - & f_{2}\end{array}$

… $\rho^{*}$

- $\pi \pi$

- - $\rho_{3} *$

SMASH-1.8 (total)

+ data (total)

+ data (elast)

Figure 2.4: $p p, p \pi^{-}$and $\pi^{+} \pi^{-}$cross-sections. Figures produced with [225] and SMASH2.0.1 [213]. 
cross-section are able to match the experimental measurements, the result for three central cross-sections ( $p p, p \pi^{-}$and $\pi^{+} \pi^{-}$) are depicted in Figure 2.4. The different interaction categories contributing are shown for $p p$ and $p \pi^{-}$, whereas for $\pi^{+} \pi^{-}$the different resonance states contributing are specifically separated. In both cases, the partial contributions combine to a total cross-section that matches the experimental data.

\section{Decay treatment and collisional broadening}

The probability of a decay in a certain timestep $\Delta t$ in the decaying resonance's rest-frame is given as

$$
P_{\text {decay }}=\frac{\Delta t}{\tau},
$$

where the lifetime $\tau$ is the inverse of the total decay width. The decay probability can either be derived assuming an exponential survival probability or directly from the Boltzmann equation. The latter is demonstrated in Section 4.1.8. The decay probability is used as the basis for Monte-Carlo decisions whether the resonance should decay in a certain timestep, such that on average, the lifetime $\tau$ of a resonance is reproduced. If the resonance decays, a decay channel of one of the contributing partial widths (cf. Eq. 2.3) is chosen according to the branching ratio $\left(\Gamma_{\text {partial }} / \Gamma_{\text {total }}\right)$ of the channel.

Since the decay width is always based on vacuum properties, its value does not change inside a hadronic medium in SMASH. However, transport approaches include an intrinsic dynamic shortening of the lifetime, which is referred to as collisional broadening. In contrast to reactions in the vacuum, in a dense hadronic medium, resonances can scatter with another particle inelastically before they decay. This effectively reduces the lifetime, which can be translated into an effective broadening of the width and, consequently, of the spectral function. Since this dynamical effect is intrinsically included in transport approaches, they allow disentangling such effects from explicit e.g. density or temperaturedependent changes to the resonance spectral shapes.

\section{Elastic collisions}

The elastic cross-sections of most relevance are experimentally well known and therefore directly parametrized. A parametrized elastic cross-sections exists for nucleon-nucleon [226], nucleon-anti-nucleon [106], nucleon-kaon [2], (anti-)deuteron-pion [135] and (anti-)deuteron(anti-)nucleon [135] scatterings. Additionally, several quasi-elastic processes model the elastic cross-section contribution via resonance formation and decay at low $\sqrt{s}$. For example, $\pi \pi$ scatters quasi-elastically via $\pi \pi \rightarrow\{\sigma, \rho\} \rightarrow \pi \pi$ and $\pi N$ via $\pi N \rightarrow \Delta \rightarrow \pi N$. As mentioned in Section 2.1.1, the $\sigma$ resonance properties are tuned to reproduce the $\pi \pi$ elastic cross-section via this quasi-elastic process.

For the remaining processes elastic cross-sections are poorly constrained by experimental data. Therefore, the cross-section for elastic processes of other baryon-baryon, baryonmeson and meson-meson processes is derived from the known $N N, N \bar{N}$ and $\pi N$ crosssection. This empirical prescription is known as the Additive Quark Model (AQM) [227] and is also used for inelastic scatterings. The same scaling as employed in UrQMD is followed [106]. Note that at high $\sqrt{s}$ energies, where no resonances are available to mediate quasi-elastic processes, the elastic $\pi \pi$ and $\pi N$ are also directly parametrized with known high energy cross-sections.

For elastic scatterings an anisotropic angular distribution is employed based on the $N N$ distribution from [228]. 


\section{Inelastic collisions}

Three inelastic collisions are treated individually in SMASH:

- Nucleon-nucleon collisions: Single resonance excitation via $N N \rightarrow N R$ with $R=$ $\Delta, N^{*}, \Delta^{*}$ and double resonance excitation via $N N \rightarrow \Delta \Delta, N N \rightarrow \Delta \Delta^{*}, N N \rightarrow$ $\Delta N^{*}$

- Nucleon-anti-nucleon collisions: Annihilation via $N \bar{N} \rightarrow h_{1} \rho$ as an effective approach for $N \bar{N} \rightarrow 5 \pi$ (discussed separately, together with the reverse reaction, in Section 4.3.3)

- Nucleon-kaon collisions: Charge exchange via $K N \rightarrow K N$, strangeness exchange via $K N \rightarrow \pi Y$ with hyperons $Y=\Lambda, \Sigma, \Xi$ and resonance excitation $K N \rightarrow K \Delta$

The $N N$ cross-sections are defined with parametrized matrix elements $|\mathcal{M}|^{2}$ that are only dependent on the Mandelstam- $s$ of the process. The cross-section in the double resonance excitation case is, therefore, given as

$$
\begin{aligned}
\sigma_{a b \rightarrow R_{1} R_{2}}(s) & =\frac{\left(2 J_{R_{1}}+1\right)\left(2 J_{R_{2}}+1\right)}{s\left|\vec{p}_{i}\right|} \sum_{I}\left(C_{a b}^{I} C_{R_{1} R_{2}}^{I}\right)^{2} \frac{|\mathcal{M}|_{a b \leftrightarrow R_{1} R_{2}}^{2}(s, I)}{16 \pi} \\
& \times \int_{m_{1}^{\text {min }}}^{\sqrt{s}-m_{2}^{\text {min }}} d m_{1} \mathcal{A}_{1}\left(m_{1}\right) \int_{m_{2}^{\text {min }}}^{\sqrt{s}-m_{1}^{\min }} d m_{2} \mathcal{A}_{2}\left(m_{2}\right)\left|\vec{p}_{f}\right|\left(\sqrt{s}, m_{1}, m_{2}\right) .
\end{aligned}
$$

Here, $\left|\vec{p}_{i(f)}\right|$ is the center-of-mass momentum of the initial (final) state, $J$ the spin of the particles, $C$ are the isospin Clebsch-Gordan factors and $\mathcal{A}_{1 / 2}$ the respective spectral function. The case of the single resonance excitation is obtained by replacing one of the spectral functions with a $\delta$ spectral function, which collapses one of the integrals. The matrix element necessary for the calculation of the $N N \rightarrow N \Delta$ cross-section is based on a fit to a one-boson-exchange model calculation [229]. The paramterization (fit parameter $A=68, b=1.104 \mathrm{GeV}$ and $c=1.951$ ) reads

$$
\frac{|\mathcal{M}|^{2}(s)}{16 \pi}=\frac{A}{(\sqrt{s}-b)^{c}} .
$$

For the other $N N$ cases the matrix element is parametrized (similar to [106]) as

$$
\frac{|\mathcal{M}|^{2}}{16 \pi}=\frac{A_{I}}{2\left(m_{a}^{2}+m_{b}^{2}\right)}
$$

with the isospin- and reaction-dependent parameter $A_{I}$ and the masses of the incoming particles $m_{a / b}$. The parameters for the matrix elements are given in Table 2.1. Note that the case of $N N \rightarrow N N^{*}(1535)$ is adapted in this work to match the experimental data (see also Section 3.1.1): The $A_{I}$-parameter is updated to $A_{I=0}=91$ for $N N \rightarrow N N^{*}(1535)$.

The strangeness exchange in kaon-nucleon scattering $(\bar{K} N \rightarrow \pi K)$ is mostly realized via a hyperon resonance state $Y^{*}\left(\pi K \rightarrow Y^{*} \rightarrow \bar{K} N\right)$. However, to describe the inelastic cross-section fully, an additional inelastic non-resonant contribution is added with the cross-section

$$
\sigma_{\bar{K} N \rightarrow \pi Y}(\sqrt{s})=\frac{A}{(\sqrt{s}-B)^{2}}
$$

where $A$ and $B$ are free parameters. This parametrization is taken from [230] with updates to the parameters described in [2]. For the charge exchange e.g. via $K^{-} p \leftrightarrow \bar{K}^{0} n$, the 


\begin{tabular}{lcc}
\hline process & $A_{I=1}$ & $A_{I=0}$ \\
\hline$N N \rightarrow N \Delta^{*}$ & 15 & - \\
$N N \rightarrow \Delta \Delta$ & 45 & 120 \\
$N N \rightarrow \Delta N^{*}$ & 7 & - \\
$N N \rightarrow \Delta \Delta^{*}$ & 15 & 25 \\
$N N \rightarrow N N^{*}\left(\right.$ except $\left.N^{*}(1535)\right)$ & 4.5 & 14 \\
$N N \rightarrow N N^{*}(1535)$ & 4.5 & 91 \\
\hline
\end{tabular}

Table 2.1: Parameters for the matrix element in Eq. 2.21 (in units of $\mathrm{mbGeV}^{4}$ ).

cross-section prescription from GiBUU [109] is followed. The $K N \rightarrow K \Delta$ cross-section is defined as the remainder of the total $K N$ cross-section after subtracting the elastic and charge exchange contributions.

Inelastic collisions in general are treated isotropically. The exception is the single resonance excitation in $N N \rightarrow N R$ with $R=\Delta, N^{*}, \Delta^{*}$. For the $\Delta$ excitation, the same treatment as for $N N$ elastic is used [228]. For $R=N^{*}, \Delta^{*}$, the Ansatz $d \sigma / d t \propto t^{-a}$ is fitted to data from [231].

Following the principle of detailed balance and imposing time-reversal symmetry for the matrix elements (Eq. 1.3) the cross-section of the reverse processes of resonance absorption can be expressed using the same matrix element as the resonance production (see [109]):

$$
\sigma_{c d \rightarrow a b}(s)=\left(2 J_{a}+1\right)\left(2 J_{b}+1\right) \frac{S_{c d}}{S_{a b}}\left|\frac{\vec{p}_{f}}{\vec{p}_{i}}\right| \frac{1}{s} \sum_{I}\left(C_{a b}^{I} C_{c d}^{I}\right)^{2} \frac{|\mathcal{M}|_{a b \leftrightarrow c d}^{2}(s, I)}{16 \pi}
$$

with $S$ being symmetry factors for the initial and final state.

\section{Resonance formation}

Similar to the resonance absorption, the cross-section of resonance formation $(a b \rightarrow R)$ can be calculated from the forward decay process $(R \rightarrow a b)$. The difference is that the integration over the matrix element is already performed as part of the decay width. Therefore, the cross-section is given in terms of the decay width as

$$
\sigma_{a b \rightarrow R}(s)=\frac{2 J_{R}+1}{\left(2 J_{a}+1\right)\left(2 J_{b}+1\right)} S_{a b} \frac{2 \pi^{2}}{\vec{p}_{i}^{2}} \Gamma_{a b \rightarrow R}(s) \mathcal{A}_{R}(\sqrt{s})
$$

This formula is already cited in [109], but the relation is also derived in Section 4.1.9 as a side product of the collision probability calculation for resonance formations.

Note that $\Gamma_{a b \rightarrow R}(s)$ only equals $\Gamma_{R \rightarrow a b}(s)$ for stable particles. For unstable incoming particles, a modified "in-width" is employed in the Manley resonance treatment. The modification reads

$$
\Gamma_{a b \rightarrow R}(m)=\Gamma_{R \rightarrow a b}^{0} \frac{\left|\vec{p}_{a b}\right| B_{L}^{2}\left(\left|\vec{p}_{a b}\right| R\right) \mathcal{F}_{a b}(m)}{m \rho_{a b}\left(M_{0}\right)}
$$

where the notation matches the one introduced for the decay width in Eq. 2.4. 


\section{String fragmentation}

The hadron-hadron cross-section for high $\sqrt{s}$ is not possible to describe with excitation of known resonance states since they are experimentally only constrained up to a mass of $\approx 2 \mathrm{GeV}$. Therefore, for the high-energy cross-section, the ideas of the Lund string model [232] are employed: If two strongly interacting particles are separated, a color flux tube (string) is formed. Due to the nature of the strong coupling constant, the energy of the string rises the larger the separation. Until at some point, enough energy for a new quark-anti-quark pair is available. The string fragments at this point and the new quarks are confined into new hadrons ${ }^{13}$. String fragmentation in this way models the multi-particle production at high-energy hadron collisions. This multi-particle production from the fragmentation of strings is handled by PYTHIA [233]. The string routine in SMASH is in detailed introduced in [200]

The string treatment of the cross-section is generally used when the scattering's $\sqrt{s}$ is larger than the sum of the masses of the colliding hadrons plus $0.9 \mathrm{GeV}$. Before this threshold, a transition region with a width of $1 \mathrm{GeV}$ between resonance and string cross-section treatment is applied. In some special cases, the transition is adapted [200]. Two different string processes are distinguished based on energy: soft and hard strings. The total and elastic cross-sections employed are parameterizations of experimental measurements. The inelastic cross-section is filled with the string cross-section. Hard strings use cross-sections calculated with pQCD [233], which only are applicable for highly energetic collisions. To interpolate between resonance and hard string cross-section in the intermediate energy range, a soft string routine is applied to match the total cross-section. The soft string treatment is similar to the one from UrQMD [106, 234]. If the inelastic cross-section for a particle pair is not known, the Additive Quark Model (AQM) [227] is employed. Similarly, since PYTHIA only fragments (anti-) nucleons and pions, other particle species are mapped onto the known fragmentations. As part of the string fragmentation routine, also $B \bar{B}$ annihilation reactions are treated as in [106, 234]. While for $N \bar{N}$ annihilations, two alternative treatments exist in SMASH (Section 4.3.3), for all other baryons, this (detailed-balance breaking) treatment is the only way to account for the annihilation reaction.

Note that the production of multiple particles in 2-hadron scatterings explicitly breaks detailed balance since the back-reaction is unaccounted for. Even though multi-particle reactions to restore detailed balance for $2 \rightarrow m$ reactions are introduced in this work, the breaking of detailed balance with strings remains since no general treatment for $m \rightarrow 2$ is introduced. Only specific $m \rightarrow 2$ reactions are accounted for because every multi-particle reaction is introduced individually and specific for the involved particles. Furthermore, PYTHIA is (conceptually) unable to reverse the fragmentation i.e. cannot reform the string from the final hadrons.

\section{Pauli Blocking}

The quantum statistics of the fermionic or bosonic particles, which is included in the quantum analog of the Boltzmann equation, the Boltzmann-Uehling-Uhlenbeck (BUU) equation, is accounted for in transport models by so called Pauli blocking. The difference between B.-eq. and the BUU-eq. are additional factors in the collision integral, which for

\footnotetext{
${ }^{13}$ The described string breaking is a way of explaining confinement in general.
} 
the BUU case reads

$$
\left(\frac{\partial f}{\partial t}\right)_{\text {coll }}=\int \frac{d^{3} p_{2}}{(2 \pi)^{3}}\left(f_{1}^{\prime} f_{2}^{\prime}\left(1 \pm f_{1}\right)\left(1 \pm f_{2}\right)-f_{1} f_{2}\left(1 \pm f_{1}^{\prime}\right)\left(1 \pm f_{2}^{\prime}\right)\right) v_{r e l} \int d \sigma
$$

Note the additional $\left(1 \pm f_{i}\right)$ suppression (enhancement) factors compared to the derived classical analogue in Eq. 4.11 in Section 4.1.2. The sign is + for bosons (boson enhancement) and - for fermions (Pauli blocking). Only Pauli blocking is implemented in SMASH by blocking reactions, where fermions would scatter into a phase space already occupied by another fermion. Numerically realized is this by rejecting scatterings with the probability $1-\prod_{i}\left(1-f_{i}\right)$, where $f_{i}$ is the phase space density for all final particle species $i$. The Pauli blocking effect is neglected for all results presented in this thesis.

\section{$2.3 \quad$ Initial conditions}

SMASH can calculate the evolution of the hadron gas in five different scenarios, which are generally distinguished by their initial condition. They are described in the following subsections.

\section{Collider}

In low energy nuclear collision, the degrees of freedom remain hadronic and the dynamics is therefore captured by a hadronic transport approach. The two colliding nuclei are represented by collections of hadrons, in general protons and nucleons according to mass and charge number of the element. Also, elementary and, in principle, exotic nuclei collisions are possible. The nucleons are distributed according to a Woods-Saxon distribution.

$$
\frac{d N}{d^{3} r}=\frac{\rho_{0}}{\exp \left(\frac{r-r_{0}}{d}\right)+1}
$$

$\rho_{0}=0.168 \mathrm{fm}^{-3}$ and $r_{0}$ are the ground state density and radius (when $d \rightarrow 0$ ). $d$ is the diffusiveness of the nucleus and by default set to $d=0.545 \mathrm{fm}$. For some nuclei specific values are set for $r_{0}$ and $d$ (see [98]). Note that also deformed nuclei are described by SMASH by skewing the Woods-Saxon distribution with an angular dependent $r=$ $r(\theta, \phi)[235]$. The nucleus centers and the starting time are chosen such that the spheres with radius $r_{0}+d$ touch at $t=0$. The distance of the centers in $x$-direction matches the impact parameter $b$.

Before the momenta are boosted in beam direction according to the configured collision energy, the nucleons get (optionally) additional Fermi motion. Since the fermionic nucleons cannot all occupy the same phase space inside the nucleus (Pauli principle), the different momentum states are uniformly filled in the ground state up to the so called Fermi momentum $p_{F}$. The Fermi momentum is defined as

$$
p_{F}(\vec{r})=\hbar c\left(3 \pi^{2} \rho(\vec{r})\right)^{1 / 3}
$$

with $\rho(\vec{r})$ being the density of nucleons at the point $\vec{r}$. Since the fermi momenta result in a sizable contribution to the measured transverse momentum $\left(p_{F} \approx 300 \mathrm{MeV}\right)$, they are include in the nucleus initial condition (when they are configured). The additional Fermi motion to the nucleons is assigned isotropically and uniformly up to $p_{F}$. The additional 
momentum leads to an exploding nucleus. Therefore, either potentials are necessary to keep the nucleus stable [98], or Fermi motion must be configured as "frozen". "Frozen" fermi motion applies the additional $\vec{p}$ only to interactions, but not to the propagation of the nucleons.

\section{Infinite Matter (Box)}

Infinite matter is simulated by imposing periodic box-shaped boundary conditions in the calculations. The box scenario is the only case where boundary conditions are imposed. Otherwise, particles can freely propagate.

The initial condition for the particle numbers inside the box is either manually configured or sampled according grand-canonical thermal multiplicities $N^{t h}\left(T, \mu_{B}, \mu_{S}, \mu_{Q}\right)$ (same equation as in Eq. 5.2), where $T$ and $\mu$ are given calculation parameters. The momenta are also thermally distributed according to

$$
w(\vec{p}) \propto \exp \left(-\sqrt{\vec{p}^{2}+m^{2}} / T\right) p^{2} d p \sin \theta d \theta d \varphi .
$$

with the probability $w$ to generate momentum $\vec{p}$. Resonances are sampled at their pole mass.

\section{Sphere}

The scenario of an expanding spherical hadron gas is similar to the one produced in a heavy-ion collision. It is, therefore, instructive to simulate a scenario with controlled initial conditions, where hadrons are uniformly distributed in a sphere with a given radius $R$. The initial particle numbers and momenta are distributed the same as for the box. The sphere expands freely due to the assigned momenta.

Note that it is additionally possible to add a high energy particle at the center of the sphere as well as the box scenario to emulate the traversing of a jet through a hadronic medium [201].

\section{Afterburner}

To employ SMASH as an afterburner of a hydro calculation for the late hadronic stages of a high-energy heavy-ion collision, the evolution is started from an initial list of hadrons. This list is created by performing a Cooper-Frye sampling ${ }^{14}$ on the hydrodynamical hypersurface. The listed particles can have different times of production. In this case, the initial state is scrolled back along their momenta to the earliest time. Particles are prevented from interacting before their production time.

\section{Initial condition for hydro}

A full integration of a hydro-phase into SMASH is recently introduced with the SMASHvHLLE-Hybrid [237]. As described, SMASH is able to be employed as an afterburner. Introduced with version SMASH-2.0 [213] is the possibility to generate initial conditions for hydrodynamic calculations. For this, a nucleus-nucleus collision is initialized as in the collider scenario. Instead of evolving the system until freeze-out, an output is generated containing a list of particles on a hypersurface of chosen constant proper time. This is

\footnotetext{
${ }^{14}$ As Cooper-Frye sampling from hypersurfaces is not included in SMASH itself, other approaches have to be employed, e.g. [236]
} 
then used as the input for hydrodynamics [91], which in turn feeds a SMASH afterburner calculation for the late stages of the hybrid calculation.

\subsection{Dilepton production}

Two electromagnetic probes are produced in SMASH: dileptons and photons. For photons, the emission in binary scatterings of mesons is of most interest. In SMASH photons are either produced by $\pi+\pi$ or $\pi+\rho$ scattering, including the Bremsstrahlung process $\pi+\pi \rightarrow \pi+\pi+\gamma$ [238]. The reader is referred to [196] for a full description of the photon treatment, as the focus of this thesis is solely on the dilepton production.

Dileptons in SMASH are produced by direct or Dalitz decays of resonances (Table 2.2). The vector mesons $\rho, \omega$ and $\phi$ decay directly into a lepton pair. Therefore, the invari-

\begin{tabular}{c}
\hline$\rho \rightarrow e^{+} e^{-}$ \\
$\omega \rightarrow e^{+} e^{-}$ \\
$\phi \rightarrow e^{+} e^{-}$ \\
\hline$\pi \rightarrow e^{+} e^{-} \gamma$ \\
$\eta \rightarrow e^{+} e^{-} \gamma$ \\
$\eta^{\prime} \rightarrow e^{+} e^{-} \gamma$ \\
$\omega \rightarrow e^{+} e^{-} \pi^{0}$ \\
$\phi \rightarrow e^{+} e^{-} \pi^{0}$ \\
$\Delta^{+} \rightarrow e^{+} e^{-} p$ \\
$\Delta^{0} \rightarrow e^{+} e^{-} n^{0}$ \\
\hline
\end{tabular}

Table 2.2: Direct and Dalitz dielectron decays

ant mass of the pair equals the mass of the resonance. Although, in principle, direct decays of either electrons and muons are implemented, the results focus on dielectrons only (Section 3.1.2). Results of the dimuon production can be found in [239]. Dalitz decays are incorporated for pseudoscalar mesons $\left(\pi, \eta, \eta^{\prime}\right)$, vector mesons $(\omega, \phi)$, and $\Delta$ baryons. All resonances are either produced by inelastic scattering, $2 \rightarrow 1$ absorption, or decays of other resonances. This also means that directly decaying resonances, like, e.g., the $\rho$ meson, include Dalitz-like contribution by coupling to baryonic resonances via processes like $N^{*} / \Delta^{*} \rightarrow \rho X \rightarrow e^{+} e^{-} X$ (see the pp results in Section 3.1.2). In addition to the modifications of the vector meson spectral functions due to the explicit coupling to baryonic degrees of freedom, collisional broadening is dynamically taken into account by construction as explained in Section 2.2.3. All dilepton decays are treated isotropically.

As SMASH combines and adapts different successful aspects of previous transport approaches in general, also the dilepton production is a mixture of previous approaches. One of the major additions is the inclusion of dilepton emission below the hadronic threshold for all direct vector meson decays. This enables the investigation of dilepton emission for the full invariant mass range, including below the hadronic invariant mass threshold. In particular, the comparison with coarse-graining results is therefore interesting. In general, the employed resonance states are most similar to the UrQMD approach [106]. Compared with the published results for the dilepton production in UrQMD [163] more recent and additional data is used for the resonance properties and cross-sections. The spectral function is also treated as in UrQMD, but the decay width treatment described above is the same as in GiBUU [109]. However, no off-shell propagation is taken into account like in HSD [240] or GiBUU. The dilepton emission is theoretically based largely 
on the GiBUU approach since it has recently proven to be successful in describing the experimental data [85]. Other transport models $[162,85]$ additionally include non-resonant production of dileptons via $\mathrm{NN}$ and $\pi \mathrm{N}$ Bremsstrahlung. Such contributions are neglected in this approach.

The two main ingredients to the dilepton treatment in SMASH are introduced in the following: the employed equations for the different decay widths and the perturbative treatment of dilepton decays. The results for the dilepton production are found in Section 3.1.2. Practical information about the usage of the SMASH dilepton routine is found in [241].

\subsubsection{Decay widths and form factors for dilepton decays}

This section details the employed decay widths for the dilepton decays. Note that an explicit parametrization is used for the decay width instead of the mass-dependent treatment from Manley et al., used for hadronic decays.

For direct decays $\Gamma_{V \rightarrow l^{+} l^{-}}\left(m_{l l}\right)$ with $V=\rho, \omega, \phi$ under the assumptions of Vector Meson Dominance [242] is

$$
\Gamma_{V \rightarrow l^{+} l^{-}}\left(m_{l l}\right)=\frac{\Gamma_{V \rightarrow l^{+} l^{-}}\left(M_{0}\right)}{M_{0}} \frac{M_{0}^{4}}{m_{l l}^{3}} \sqrt{1-\frac{4 m_{l}^{2}}{m_{l l}^{2}}}\left(1+\frac{2 m_{l}^{2}}{m_{l l}^{2}}\right)
$$

with $m_{l l}$ being the invariant mass of the lepton pair, $M_{0}$ the pole mass of the vector meson and $m_{l}$ the lepton mass. For $\Gamma_{V \rightarrow l^{+} l^{-}}\left(M_{0}\right)$ values from the PDG [243] are used. The effect of the inclusion of the dilepton decay width for the $\rho$ resonance total decay width is depicted in Figure 2.5. The $m_{l l}^{-3}$ dependence is clearly visible at low masses.

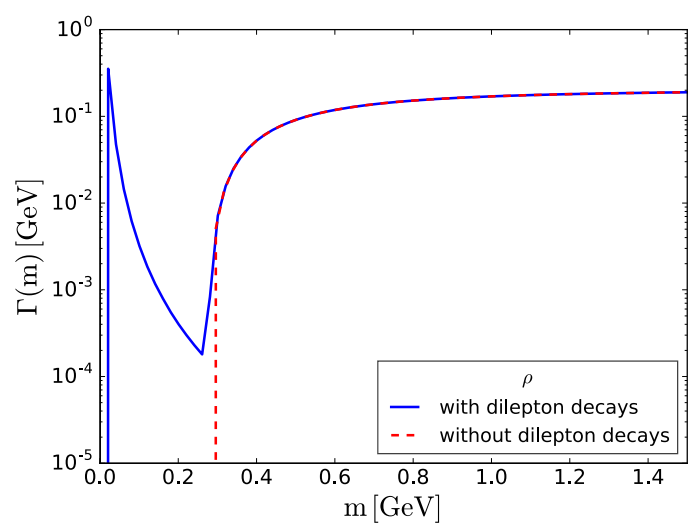

Figure 2.5: Decay widths of the $\rho$ resonance with and without the dilepton decay mode.

The contributions to the spectral function below the hadronic threshold are also observed in Figure 2.1. Note that taking such contributions into an account for all vector mesons is one of the major additions of this dilepton transport treatment.

For Dalitz decays the invariant mass of the dilepton is not fixed by the mass of the decaying resonance, because of the three decay products. Hence only a differential decay width $d \Gamma / d m_{l l}$ is given. For the pseudoscalar Dalitz decays $P=\pi^{0}, \eta, \eta^{\prime}$ the differential 
width is given by [244],

$$
\frac{d \Gamma_{P \rightarrow \gamma e^{+} e^{-}}}{d m_{l l}}=\frac{4 \alpha}{3 \pi} \frac{\Gamma_{P \rightarrow \gamma \gamma}}{m_{l l}}\left(1-\frac{m_{l l}^{2}}{m_{P}^{2}}\right)^{3}\left|F_{P}\left(m_{l l}\right)\right|^{2}
$$

with $\Gamma_{\pi^{0} \rightarrow \gamma \gamma}=7.6 \cdot 10^{-6} \mathrm{MeV}, \Gamma_{\eta \rightarrow \gamma \gamma}=5.2 \cdot 10^{-4} \mathrm{MeV}$ and $\Gamma_{\eta^{\prime} \rightarrow \gamma \gamma}=4.4 \cdot 10^{-3} \mathrm{MeV}$ [243], $\alpha=1 / 137$ and $m_{P}$ the mass of the pseudosalar meson. The form factors $F_{P}$ are

$$
\begin{aligned}
& F_{\pi^{0}}\left(m_{l l}\right)=1+b_{\pi^{0}} m_{l l}^{2}, \quad b_{\pi^{0}}=5.5 \mathrm{GeV}^{-2}, \\
& F_{\eta}\left(m_{l l}\right)=\left(1-\frac{m_{l l}^{2}}{\Lambda_{\eta}^{2}}\right)^{-1}, \quad \Lambda_{\eta}=0.716 \mathrm{GeV}
\end{aligned}
$$

with $\Lambda_{\eta}$ taken from [245]. For the $\eta^{\prime}$ form factor the QED approximation $F_{\eta^{\prime}}\left(m_{l l}\right)=1$ is used. The vector-meson Dalitz decays $(V=\omega, \phi)$ are parametrized by $[244,246]$,

$$
\frac{d \Gamma_{V \rightarrow \pi^{0} e^{+} e^{-}}}{d m_{l l}}=\frac{2 \alpha}{3 \pi} \frac{\Gamma_{V \rightarrow \pi^{0} \gamma}}{m_{l l}}\left[\left(1+\frac{m_{l l}^{2}}{m_{V}^{2}-m_{\pi}^{2}}\right)^{2}-\frac{4 m_{V}^{2} m_{l l}^{2}}{\left(m_{V}^{2}-m_{\pi}^{2}\right)^{2}}\right]^{3 / 2}\left|F_{V}\left(m_{l l}\right)\right|^{2},
$$

where $m_{V}$ is the mass of the vector meson, $m_{\pi}$ the pion mass and

$$
\left|F_{\omega}\left(m_{l l}\right)\right|^{2}=\frac{\Lambda_{\omega}^{4}}{\left(\Lambda_{\omega}^{2}-m_{l l}^{2}\right)^{2}+\Lambda_{\omega}^{2} \Gamma_{\omega}^{2}} .
$$

The other parameters are set as follows: $\Gamma_{\omega \rightarrow \pi^{0} \gamma}=0.703 \mathrm{MeV}, \Gamma_{\phi \rightarrow \pi^{0} \gamma}=5.4 \mathrm{keV}$ [243], $\Lambda_{\omega}=0.65 \mathrm{GeV}$ and $\Gamma_{\omega}=75 \mathrm{MeV}$ [246]. For the $\phi$ form factor the QED approximation $\left|F_{\phi}\left(m_{l l}\right)\right|^{2}=1$ is chosen. Note, that in previous work [247] the possibility of describing these decays, similar as the hadronic $V \rightarrow 3 \pi$ decays, in two steps via an intermediate $\rho$ meson $V \rightarrow \pi \rho \rightarrow \pi e^{+} e^{-}$has been explored. This would render the parameterizations given above obsolete and remains an appealing option for the future. For this work, the more established direct treatment has been chosen, which also allows a more direct comparison to similar approaches $[85,163]$ that rely on the same formalism.

For the $\Delta$ Dalitz decay, the differential decay width by Krivoruchenko et al. [248] is applied,

$$
\begin{gathered}
\frac{d \Gamma_{\Delta \rightarrow N e^{+} e^{-}}}{d m_{l l}}=\frac{2 \alpha}{3 \pi} \frac{\Gamma_{\Delta \rightarrow N \gamma^{*}}\left(m_{l l}\right)}{m_{l l}}, \\
\Gamma_{\Delta \rightarrow N \gamma^{*}}\left(m_{l l}\right)=\frac{\alpha}{16} \frac{\left(m_{\Delta}+m_{N}\right)^{2}}{m_{\Delta}^{3} m_{N}^{2}}\left[\left(m_{\Delta}+m_{N}\right)^{2}-m_{l l}^{2}\right]^{1 / 2} \\
\quad \times\left[\left(m_{\Delta}-m_{N}\right)^{2}-m_{l l}^{2}\right]^{3 / 2}\left|F_{\Delta}\left(m_{l l}\right)\right|^{2} .
\end{gathered}
$$

The form factor $\left|F_{\Delta}\left(m_{l l}\right)\right|^{2}$ is a topic of ongoing debate [249, 250]. For this work, it is chosen to be constant and fixed at the photon point $F_{\Delta}(0)=3.12 \equiv F_{\Delta}\left(m_{l l}\right)$, where it is known that $\Gamma_{\Delta \rightarrow N \gamma}(0)=702 \mathrm{MeV}[243]$.

\subsubsection{Shining method}

In experiment and numerical simulations, a major challenge of electromagnetic probes is their rare production. For dileptons, the decay branching ratios are small, typically on 
the order of $10^{-5}$. The reason is that the electromagnetic coupling constant is orders of magnitudes smaller than the strong coupling constant. They are therefore treated perturbatively i.e. the decays are only performed virtually (producing a separate output) and do not affect the hadronic evolution itself ${ }^{15}$, which is a justified approximation due to the difference in the coupling strength. Such a perturbative treatment neglects any secondary interactions of the leptons after the decay, e.g. by the Coulomb force. This is also justified since leptons only interact via the weak electromagnetic interaction and are not perturbed by strong interactions.

The perturbative treatment of leptons follows the so called Time Integration Method, also referred to as the Shining Method [251, 252]. The idea is to obtain the dilepton yield $\Delta N_{l^{+} l^{-}}$by integrating the decay probability of the dilepton decay mode $\Gamma_{l^{+} l^{-}} d t$ over the Lorentz-corrected $(\gamma)$ lifetime $\tau=t^{\dagger}-t^{*}$ of a given resonance:

$$
\Delta N_{l^{+} l^{-}}=\int_{t^{*}}^{t^{\dagger}} \frac{d t}{\gamma} \Gamma_{l^{+} l^{-}}
$$

This is done numerically by continuously emitting (shining) dileptons during the propagation of a resonance and weighting them by taking their decay probability into account. The (shining) weight $w_{s h}$ for a dilepton pair that is emitted over a timestep of $\Delta t$ is given by the integrand of the integral above as

$$
w_{s h}=\frac{\Delta t}{\gamma} \Gamma_{l^{+} l^{-}} .
$$

Since for Dalitz decay, only a differential $\frac{d \Gamma}{d m_{l}}$ is available, the integration over the invariant mass is implicitly Monte-Carlo sampled by using the following shinning weight for Dalitz decays.

$$
w_{s h}^{\text {Dalitz }}=\frac{\Delta t}{\gamma} \frac{d \Gamma}{d m_{l l}} \Delta m_{l l}
$$

$\Delta m_{l l}$ is the available invariant mass range for the dilepton mass i.e. the result of subtracting the non-leptonic and twice the electron mass from the resonance mass. Should a resonance be present at the end of the calculation. A dilepton decay of it is weighted by the branching ratio.

\subsubsection{Coarse-graining approach}

Coarse-graining [114] extracts event-averaged macroscopic quantities locally for small space-time cells. Dileptons are produced by those cells according to thermal emission rates. The framework used in this work is the same as in [117] and has proven to be reliable in describing experimental data from SIS up to LHC energies [117, 116, 118, 253]. In the following, a summary of the approach is given. A full review is found in [117]. The coarse-graining approach is strictly speaking not part of the SMASH approach itself

\footnotetext{
${ }^{15}$ The perturbative dilepton treatment causes the numerical artifact that some particles only have a dilepton decay channel and are, therefore "hadronically stable". This includes vector mesons with a mass below the hadronic threshold. Such particles are excluded from the shinning treatment until the end of the calculation since they live indefinitely. Instead of emitting dileptons during their propagation, their decay is counted (once) at the end. To avoid that resonances with a mass below the hadronic threshold are part of the final state, they decay into the lepton pair at this point. It is verified that this artifact does not affect the presented results.
} 
since it only utilizes the generated particle output of SMASH as an input to extract the coarse-grained evolution.

The approach follows the original idea from [114] by locally averaging over the reaction evolution and splitting an ensemble of collision events into small spacetime cells. For those cells, the baryon density $\rho_{B}$ and the energy density $\epsilon$ are calculated in the restframe. Knowing both and assuming (for the low beam energies considered here) a hadron resonance gas equation of state, the local temperature $T$ and baryon chemical potential $\mu_{B}$ are determined. Based on the thermodynamic information, the yield of dileptons from a certain cell $\left(\frac{d N_{l l}}{d^{4} x d^{q}}\right)$ is given by the corresponding thermal emission rates that include the in-medium modification on the vector meson spectral function. The rates are defined in relation to the imaginary part of the electromagnetic current-current correlation function $\operatorname{Im} \Pi_{\mathrm{em}}^{(\mathrm{ret})}[254]$ as

$$
\frac{d N_{l l}}{d^{4} x d^{q}}=-\frac{\alpha^{2} L(M)}{\pi^{3} M^{2}} f^{B} \operatorname{Im} \Pi_{\mathrm{em}}^{(\mathrm{ret})} .
$$

$f^{B}$ is the Bose-distribution, $\alpha$ is the e.m. coupling constant, $M$ the mass of the resonance and $L(M)$ the lepton phase space factor.

The medium modified spectral function directly enters in this equation, since the correlator is directly related to the spectral function i.e. the imaginary part of its retarded propagator $\operatorname{Im} D_{\rho}^{\text {ret }}$ as

$$
\operatorname{Im} \Pi_{\mathrm{em}}^{(\mathrm{ret})}=\frac{m_{0}^{4}}{g^{2}} \operatorname{Im} D_{\rho}^{\mathrm{ret}}
$$

with the degeneracy factor $g$. See [116] for more details on the employed emission rates.

The in-medium description used in the coarse-grained approach is based on the hadronic many-body theory $[145,255]$, where the spectral function depends on temperature and density. The medium modification of the spectral function is illustrated in Figure 2.6.

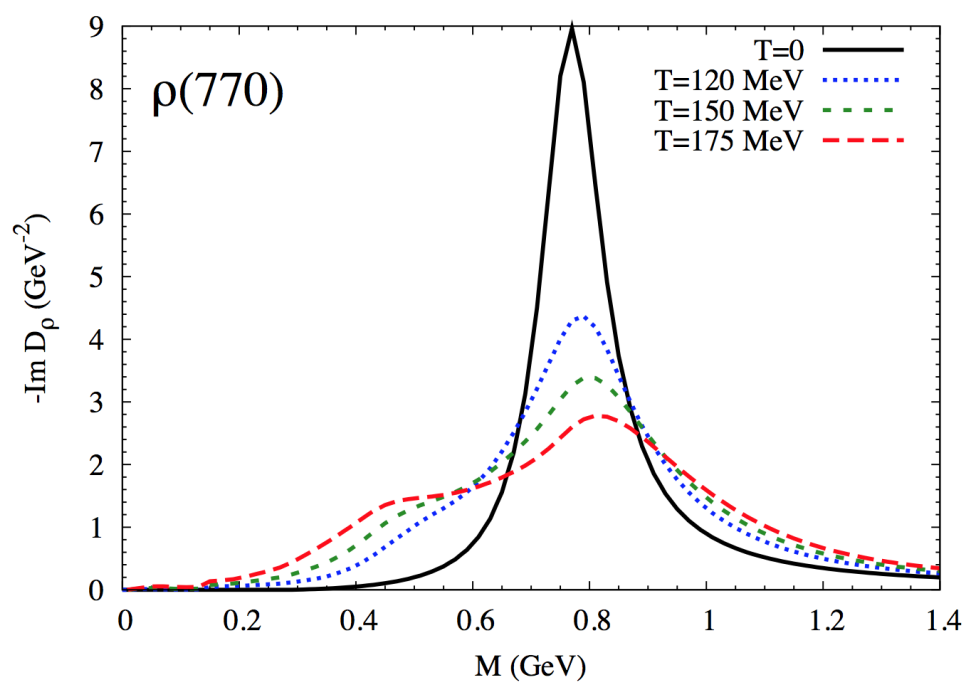

Figure 2.6: Melting in-medium spectral functions of the $\rho$ meson with rising temperature. Calculation based on a hadronic many-body theory. Figure from [136].

Since in-medium modifications at these energies are only expected to significantly affect the $\rho$ and $\omega$, dilepton yields from thermal rates are only calculated for these two. If the 
temperature drops inside the cells, the assumption of thermal rates is no longer reasonable; therefore also non-thermal (freeze-out) transport contributions are included as part of the coarse-graining model. They are only around the pole masses significant [117]. The $\omega$ Dalitz decay is also part of the $\omega$ freeze-out contribution. Thermal rates together with the freeze-out contribution result in the coarse-graining contributions for the $\rho$ and $\omega$ (CG- $\rho$ and CG- $\omega)$. The last contribution from the coarse-graining approach are multi- $\pi$ states originating from broad resonances. The dilepton cocktail is completed with the relevant transport contributions of $\pi, \eta$ and $\phi$ from SMASH. 



\section{Chapter 3}

\section{Results with binary collisions}

This chapter discusses two complementary observables that are especially sensitive to the medium evolution and its properties: dilepton and strangeness production. These rare and newly produced probes offer a perspective of special interest on the medium and complement more basal observables. They in particular are sensitive to resonance properties and particle production mechanisms (as explained in Section 1.3.1 and Section 1.3.2). The production of dileptons and strangeness is studied for a large variety of collision systems at low-beam energies. The idea is to constrain the different treatments in elementary and small systems, where in-medium effects and the likelihood of scatterings of multiple particles are expected to be small. A transport approach based on vacuum properties is expected to be compatible with the experimental data in this case. The results benchmark the approach and establish a (binary) "vacuum" baseline, which also is exploited for the multi-particle studies (in Chapter 5). For the larger collisions systems, a highly compressed and therefore very dense system is created, where the onset of medium effects is expected. Therefore, effects of the dense medium on dileptons and strangeness can be identified by differences between the transport and experimental results.

The results in this chapter only employ binary collisions. This limitation is commonly made in transport approaches. Here, it allows studying and understanding the hadron gas evolution before intervening in the reaction dynamic itself by introducing multi-particle reactions. All results in this section are produced with the geometric collision criterion. Definitions for the kinematic variables and observables used in results in this or the following chapters are e.g. found in [256]. Note that, while the later introduced multi-particle reactions are found to be essential for certain observables, e.g. the yield of deuterons or protons (see Chapter 5), the presented results for dileptons and strangeness are not sensitive to them. 

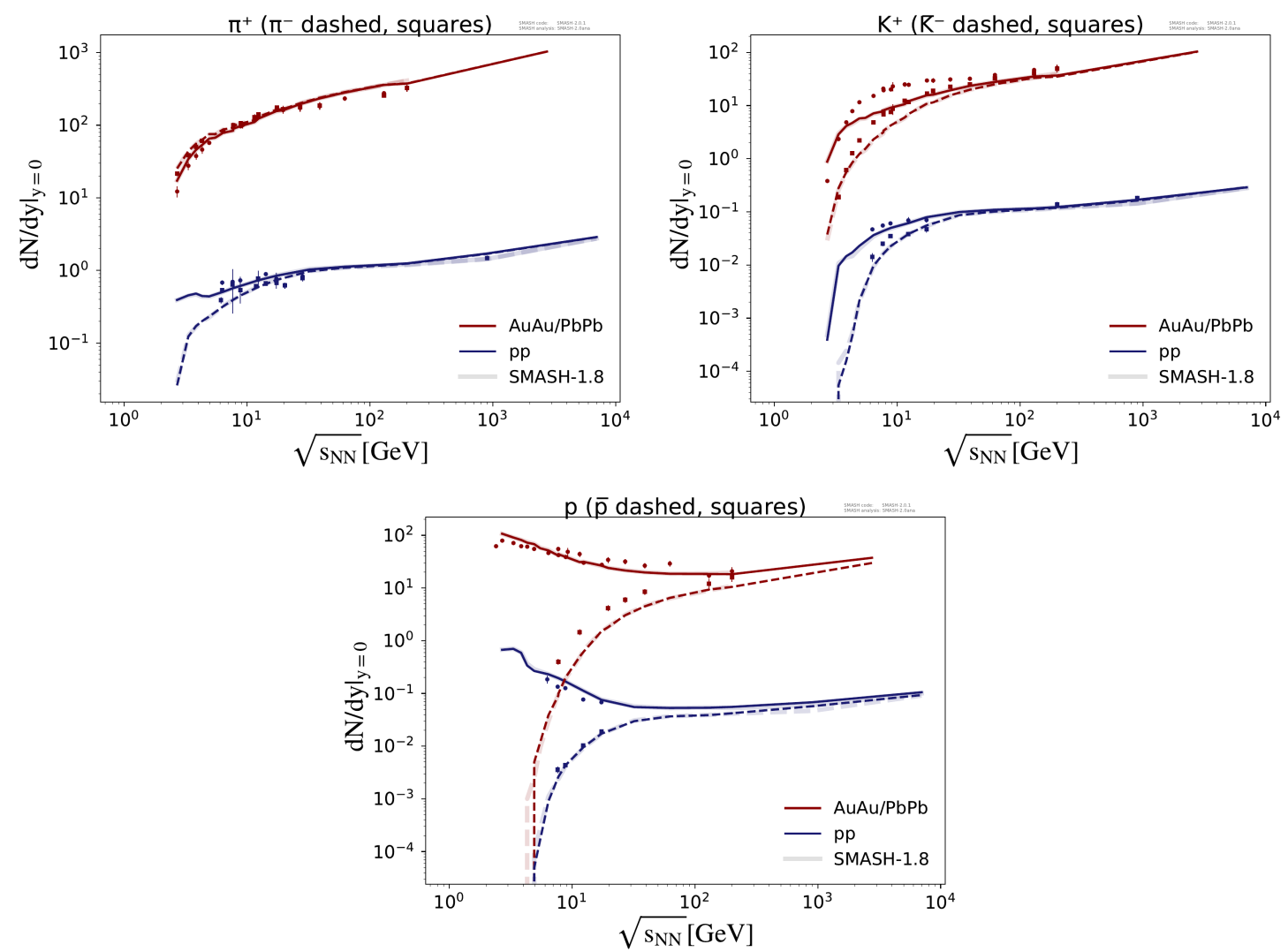

Figure 3.1: Mid-rapidity multiplicities of $\pi, K$ and $p$ for different beam energies. Results produced with [225].

As a starting point and general context for the results with binary collisions, a set of hadrons abundances produced in elementary proton-proton and heavy-ion $\mathrm{AuAu}$ or $\mathrm{PbPb}$ collisions ${ }^{1}$ with SMASH (version SMASH-2.0.1 [213]) is shown in Figure 3.1. The particle and anti-particle multiplicities are compared to experimental data at mid-rapidity for a large range of beam energies. Deviations to the heavy-ion measurements are mainly seen for $K$ and $\bar{p}$. The deviation for $K$ is discussed as part of Section 3.2. Despite the differences, the results mark a first general validation of the approach since the hadron production is mostly compatible with the experimental data, particularly in elementary collisions. Based on this general observation for hadron production, the complementary and more specific dilepton production is investigated.

\footnotetext{
${ }^{1}$ The collision system $(\mathrm{AuAu}$ or $\mathrm{PbPb})$ is chosen to match the system for which the respective experimental data point is taken.
} 


\subsection{Dilepton emission}

This section is based on [1] and [3].

Dilepton emission offers a complementary observable to hadron production and a wealth of experimental data to compare, constrain and study different aspects for the properties of the hadronic medium. Since the lepton pairs escape the created medium unperturbed, the dilepton invariant mass ${ }^{2}$ spectrum allows access to the resonance spectral function and, in turn, address the question if and how the resonances properties change inside a hot and dense medium (see also Section 1.3.1). The dilepton production in this section is discussed for elementary, nucleon-nucleus and nucleus-nucleus collisions using SMASH [98] and coarse-a graining approach [116] in the context of the whole set of HADES measurements.

Different medium effects that are closely related to the study of dileptons are a focus of the discussion. The microscopic transport includes collisional broadening (Section 2.2.3). Since absorption of resonances by other hadrons is dynamically accounted for, the lifetime is shortened in the presence of a hadronic medium. The reduction of the lifetime equals an effective broadening of the decay width of the resonance. The later employed coarse-graining approach also includes vector meson spectral functions that are explicitly dependent on the medium's temperature and density of the medium [145, 255]. Such modifications will be labeled as in-medium modifications since they are only employed for the evolution within a hot and dense medium. In this context, the coupling of baryons to the vector mesons is of particular interest [136]. In SMASH, this coupling is included as the decay of baryonic resonances into vector mesons. Whereas in the calculation of the in-medium spectral functions, the coupling enters as an important contribution to the self-energy. This is done in a self-consistent way, including interference terms. On the other hand, the presented transport calculation neglects interference terms and broadening of the spectral function originating from a consistent treatment of the self-energies in the vector meson propagator as an approximation. The reported results allow access to these medium effects since they significantly affect the dilepton invariant mass spectra by contrasting the emission of the transport and the coarse-graining approach.

In addition, the following study is motivated by the validation of the SMASH approach in general and the resonance description in particular. For this, the dilepton yield is calculated and subsequently compared to experimental data. It is, for example, possible to specifically probe and constrain the branching ratios of decays of baryonic resonances into vector mesons. The dilepton production furthermore facilitates comparisons to other approaches and predictions for newly measured collision systems by HADES [257, 258].

Compared to previous efforts for the dilepton production in transport approaches [85, $162,163]$, which establish a solid foundation for this work, two new aspects are studied here: First, low-mass contributions to the vector meson decay channels are studied for all vector mesons. The spectral function for vector mesons does not vanish at the hadronic threshold. Instead, it vanishes at $2 m_{e}$, the smallest possible invariant mass of the decay products, when including $V \rightarrow e^{+} e^{-}$decays $(V=\rho, \omega, \phi)$. The treatment consequently leads to low-mass contributions below the hadronic threshold for direct vector meson dilepton decays. These low-mass dilepton yields are investigated. Especially, their significance relative to other decay channels and for the total yield is studied. Here, all vector mesons

\footnotetext{
${ }^{2}$ The invariant mass of the dilepton is given by $m_{l l}^{2}=\left(p_{l l}^{0}\right)^{2}-{\overrightarrow{p_{l l}}}^{2}$ with $p_{l l}$ being the combined lepton pair four-momentum.
} 
$(\rho, \omega, \phi)$ are included. This is an extension of previous work with the GiBUU approach, which considered such contributions only for the $\rho$ meson. The UrQMD approach, on the other hand, neglects them entirely for numerical reasons. Secondly, the employment of a coarse-graining approach [117] in combination with the just discussed low-mass contributions offers the unique opportunity of a direct comparison. It is possible to investigate different medium effects on the dilepton spectrum, including the low-mass region, based on the same hadronic evolution. The coarse-graining approach employs in-medium spectral functions for the vector mesons, whereas the transport calculation's spectral functions are based on vacuum properties. Therefore, comparing the decay yield of the vector mesons produced by both approaches is of particular interest. Although results for a coarsegrained UrQMD dilepton production are reported [117, 253], such a direct comparison has not been performed. Additionally, the low-mass contributions are neglected for the UrQMD transport calculations, making a comparison to the corresponding coarse-grained results in this mass region unfeasible.

The results are structured as follows. First, a selection of elementary cross-sections of dilepton emitting resonances is studied. Next, the complete set of available dielectron production measurements at SIS energies is discussed in the context of the hadronic transport approach SMASH. Starting with the smallest systems created during elementary collisions and ending with the largest system studied $(\mathrm{AuAu})$. These results are then complemented and compared to the results from the employed coarse-graining approach for the $\mathrm{ArKCl}$, $\mathrm{AgAg}$ and $\mathrm{AuAu}$ system, where medium effects are expected to become relevant. The results are produced utilizing the in Section 2.4 introduced dilepton emission. If not mentioned otherwise, results are obtained using version SMASH-1.1.

\subsubsection{Elementary cross-sections of dilepton emitting particles}

Cross-section data is a, in general, valuable tool to constrain particle production for different collision energies. This section includes several results for particles that decay into dileptons. Producing the correct number of resonances in elementary reactions is essential before investigating the dilepton emission itself. The cross-section results complement the already reported total and single pion production cross-sections in [98]. Beginning with the $\eta$ production cross-section in pp collisions, Figure 3.2 shows the exclusive $\eta$ production in $p p \rightarrow p p \eta$. A good agreement is observed close to the threshold, whereas too many $\eta$ mesons are produced from $p p \rightarrow p p \eta$ for $\sqrt{s}>3.25 \mathrm{GeV}$. The disagreement, however, does not affect the few-GeV energy range of HADES measurements studied in this work. Figure 3.3 shows the exclusive $\omega$ production cross-section. SMASH results are in reasonable agreement with the relatively large error bars. In the case of the $\rho$ meson, both inclusive $(p p \rightarrow \rho+X)$ and exclusive cross-sections $(p p \rightarrow p p \rho)$ are shown (Figure 3.4). Both are overestimated (solid lines) compared to the experimental data points, especially the exclusive cross-section, which dominates the inclusive cross-section for energies close to the threshold. It is important to consider here that the $\rho$ meson in SMASH is used as an intermediate state to emulate different Dalitz decays in two steps following the idea of strict Vector Meson Dominance [263]. The advantage of this treatment is the conservation of detailed balance (see also Section 4.3.1). The two most prominent decays are the $3 \pi$ decay of the $\omega(\omega \rightarrow \rho \pi \rightarrow 3 \pi)$ and the $N^{*}(1520)$ dilepton Dalitz decay, which is emulated by $N^{*}(1520) \rightarrow \rho N \rightarrow e^{+} e^{-} N$. Neglecting these additional proxy contributions (transparent lines in Figure 3.4) leads to an agreement within errors for the inclusive cross-section data point, but not for the exclusive channel. Therefore, the overestimation in the inclusive $\rho$ production can be seen as a compromise of the two-step treatment of three-body decays. 


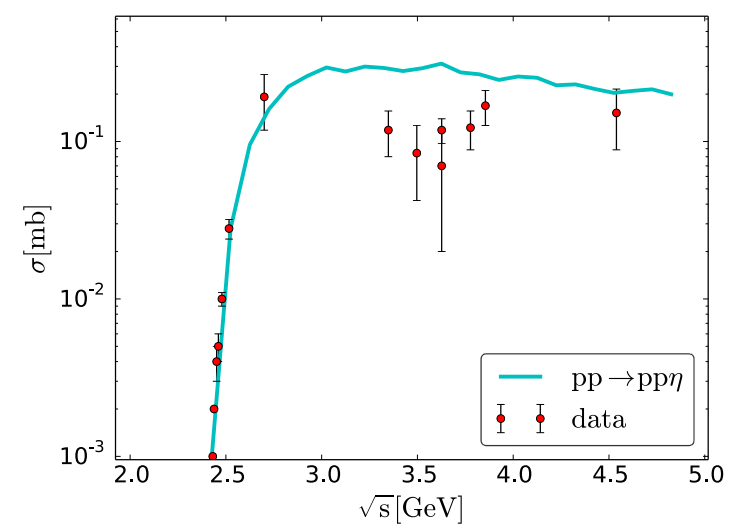

Figure 3.2: Production cross-section for $p p \rightarrow p p \eta$. Experimental data from [259, 260, 261].

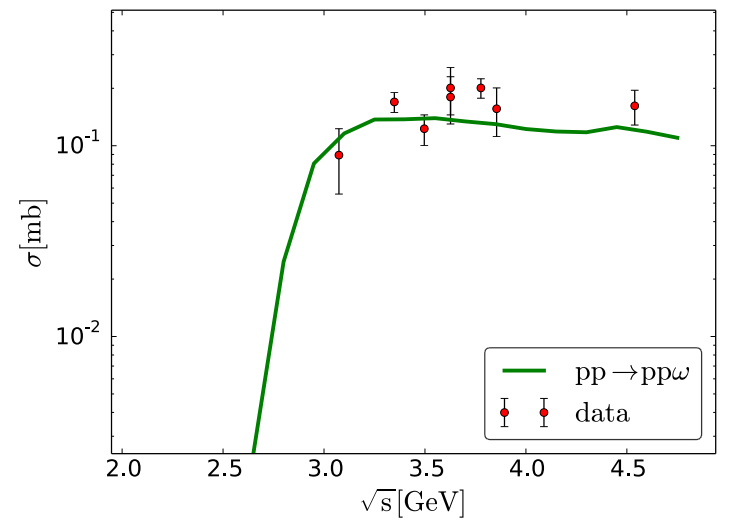

Figure 3.3: Production cross-section for $p p \rightarrow p p \omega$. Experimental data from [261].

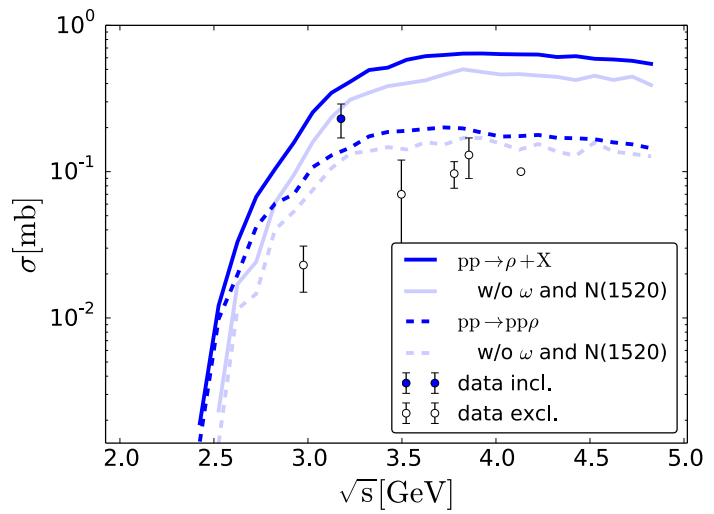

Figure 3.4: Exclusive production cross-section for $p p \rightarrow p p \rho$ (empty data points from $[262,261]$ ) and inclusive $p p \rightarrow \rho+X$ cross-section (full data point from [155]). 


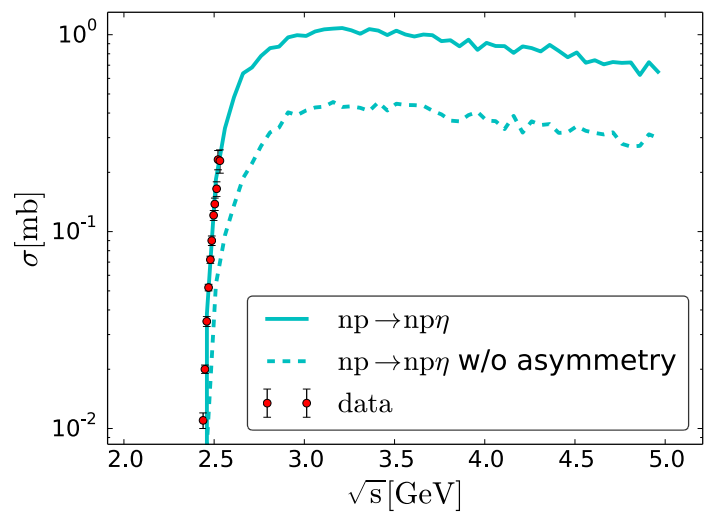

Figure 3.5: cross-section for $p n \rightarrow p n \eta$. Experimental data from [264].

\section{Isospin-asymmetric $\eta$ production}

In general, SMASH assumes isospin symmetry in the production of particles, but there are a few exceptions to this treatment for NN reactions. One is newly introduced here to improve the description of the dilepton yield stemming from the Dalitz decay of the $\eta$ meson. There exists experimental evidence that the $\eta$ production via $p n \rightarrow p n \eta$ is enhanced in comparison to $p p \rightarrow p p \eta$ by approximately a factor of 6.5 [264]. The dominant source of the $\eta$ meson for low energies is the decay of $N^{*}(1535)$. Therefore its production is enhanced following the suggestion from [265] by modifying the matrix element,

$$
\left|\mathcal{M}_{p n \rightarrow N N^{*}(1535)}\right|^{2}=6.5 \times\left|\mathcal{M}_{p p \rightarrow N N^{*}(1535)}\right|^{2} .
$$

The modification is done by adapting the parameterization of the $N N \rightarrow N N^{*}$ matrix element as described in Section 2.2.3.

This introduction of isospin asymmetry in the production of $N^{*}(1535)$ and consequently of $\eta$ mesons greatly improves agreement with experimental data from [264] as seen in Figure 3.5.

\subsubsection{Results for dilepton production}

This section is separated into different parts covering the different collision system types at low beam energies for which experimental data is available. First, elementary reactions are studied. Afterward, results for proton-nucleus collisions are discussed and the last section includes the results for nucleus-nucleus collisions.

The smaller systems (up to CC) verify the hadronic and dilepton emission baseline of the transport approach with on vacuum properties. On this basis, the dilepton emission for the larger systems ( $\mathrm{ArKCl}, \mathrm{AgAg}$ and $\mathrm{AuAu}$ ) can point to and constrain medium effects by deviations to the measurements. The study of medium effects is then expanded with the coarse-graining approach in the following section.

In general, contributions from all decay channels (Table 2.2) are taken into account in the following, but for the Dalitz decays of $\phi$ and $\eta^{\prime}$ in particular, only negligible contributions are observed for the systems studied here. Therefore, almost all results exclude both channels. The initial conditions for the proton-nucleus and nucleus-nucleus collision are given as explained in Section 2.3. All results that include experimental data from HADES 
are filtered using the HADES acceptance filter (HAFT [266]). Additionally, to match the experimental analysis procedure, an opening angle $\left(\Theta>9^{\circ}\right)$ cut and the single lepton momentum cut for the specific system have been applied.

\section{Elementary collisions}

Elementary collisions offer the possibility to constrain and test the description of the binary reactions occurring in a nucleus-nucleus collision. Therefore, they represent a baseline for the dilepton production in nucleus-nucleus reactions. Results for three different systems are shown: proton-proton, neutron-proton and $\pi$-proton. For the sake of data comparison, the same energies as measured by HADES were chosen [155, 153, 154]. Results for the pion beam are not published yet, so predictions, which are not filtered for acceptance, are shown in the following.

\section{pp}

Dilepton production in proton-proton (pp) reactions is calculated for three different kinetic energies $E_{\mathrm{Kin}}=1.25 / 2.2 / 3.5 \mathrm{GeV}$ in a fixed-target setup. Figure 3.6 shows the dilepton invariant mass spectrum for the two lower energies in comparison to HADES data [153, $154]$.
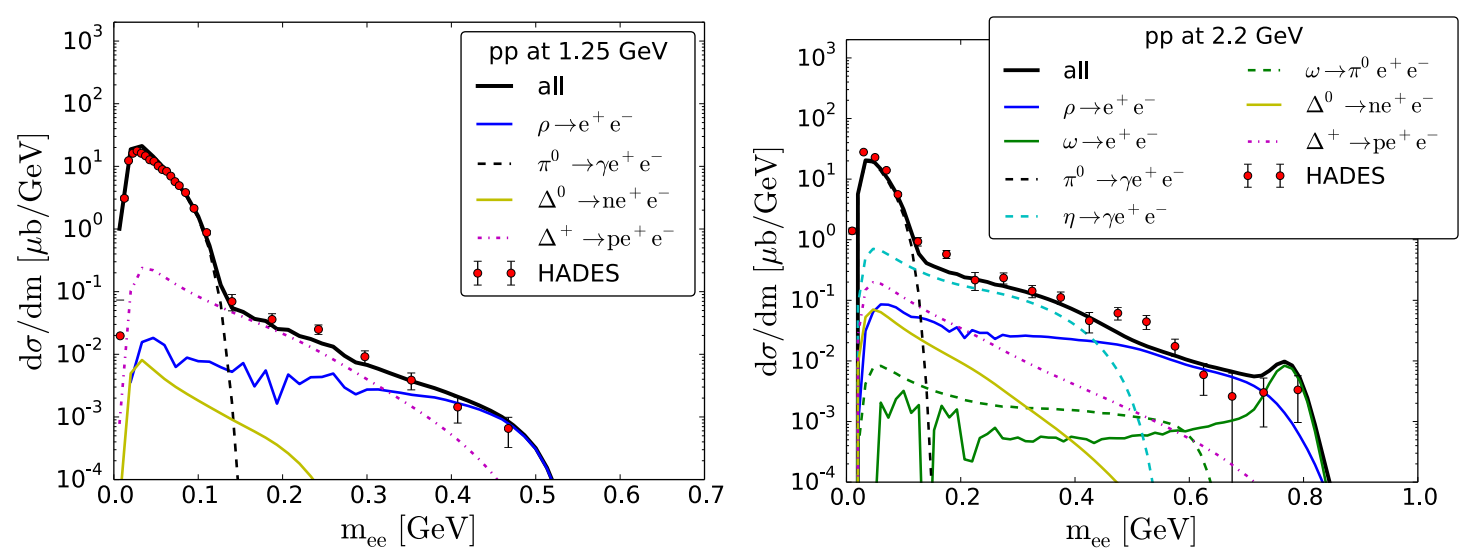

Figure 3.6: Invariant mass spectrum of dielectrons produced in pp collisions at $E_{\text {Kin }}=$ $1.25 \mathrm{GeV}$ and $E_{\mathrm{Kin}}=2.2 \mathrm{GeV}$. Experimental data from $[153,154]$.

For the reaction at $E_{\text {Kin }}=1.25 \mathrm{GeV}$ in Figure 3.6 only four different channels of the whole dilepton cocktail are contributing. The $\pi^{0}$ Dalitz decay dominates in the $\pi^{0}$ invariant mass region up to around $0.15 \mathrm{GeV}$. Above $0.15 \mathrm{GeV}$ in the low mass region, the Dalitz decay of the $\Delta^{+}$decay is dominant. Since the $\Delta^{+}$and the $\Delta^{0}$ contributions are plotted separately, a difference of more than one order of magnitude can be observed. The $\Delta^{+}$is more likely to be produced since it can be a product of the primary collision, whereas the $\Delta^{0}$ can only be formed in secondary reactions due to charge conservation and the fact that only $2 \rightarrow 2$ reactions are allowed in SMASH. In the higher invariant mass region, a large contribution from the direct $\rho$ meson channels is noticed. The total yield is in good agreement with experimental data. Since a kinetic energy of $E_{\mathrm{Kin}}=1.25 \mathrm{GeV}$ is slightly below and $E_{\mathrm{Kin}}=2.2 \mathrm{GeV}$ is above the $\eta$ production threshold, a contribution from the $\eta$ meson is seen in Figure 3.6 for $E_{\text {Kin }}=2.2 \mathrm{GeV}$. Also, additional significant contributions from $\omega$ decays are observed for the higher kinetic energy. Here, the $\eta$ yield is dominant for the invariant mass region up to around $0.4 \mathrm{GeV}$. The $\pi$ again dominates for low invariant 
masses and the $\rho$ in the mass region above $0.4 \mathrm{GeV}$. The peak in the $\omega \rightarrow e^{+} e^{-}$spectrum at the $\omega$ pole mass can already be observed for this kinetic energy. The overall agreement with data is again reasonable. Figure 3.7 shows the invariant mass spectrum produced by

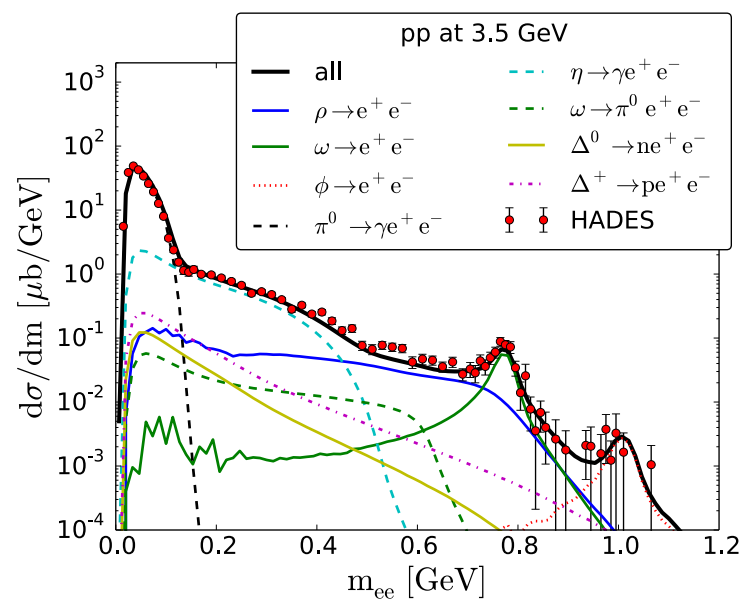

Figure 3.7: Invariant mass spectrum of dielectrons produced by pp collisions at $E_{\text {Kin }}=$ $3.5 \mathrm{GeV}$. Experimental data from [155].

fixed target pp reactions with a kinetic energy of $3.5 \mathrm{GeV}$. Because of the higher energy, the spectrum reveals two new features: a more pronounced $\omega$ peak and an additional $\phi$ contribution. The significant contributions to the spectrum now reach up to $1.1 \mathrm{GeV}$. Again, a good agreement with experimental data is observed.

In Figure 3.8 and Figure 3.9, $p_{T}$ and $y$ spectra for different invariant mass windows are shown for completeness. The lepton pair transverse momentum and rapidity from reactions at $E_{\mathrm{Kin}}=3.5 \mathrm{GeV}$ are compared to experimental data from [155] in different invariant mass windows that reflect the different dominant contributions over the invariant mass range. Below $150 \mathrm{MeV}$ the $\pi^{0}$ Dalitz decay dominates. Between $150 \mathrm{MeV}$ and $470 \mathrm{MeV}$ the $\eta$ decay is the largest contribution, while above $470 \mathrm{MeV}$ and below $700 \mathrm{MeV}$ the $\rho$ channel exceeds the others. Above $700 \mathrm{MeV}$ the $\omega$ peak is dominant. The plots in Figure 3.8 and Figure 3.9 show that also for those more differential spectra that probe specific channels and different kinematic observables that probe different regions of the phase space agreement with experimental data is reasonable.

The contributions from the direct decays of the vector mesons with masses reaching below the hadronic thresholds are important for all three energies. They originate from the resonance description discussed in Section 2.1.1 that considers the dilepton decays for the spectral function. In the case of the $\rho$ meson, those contributions are significant for the total yield in the low mass region. For the $\omega$ they are negligible compared to e.g. the $\omega$ Dalitz decay in elementary system. Nevertheless, Figure 3.7 does show that contributions below the hadronic threshold are observed for both mesons. This also holds for the direct $\phi$ decay, but low invariant mass contributions in pp are too small to be visible for the $\phi$ meson on the chosen scale. 

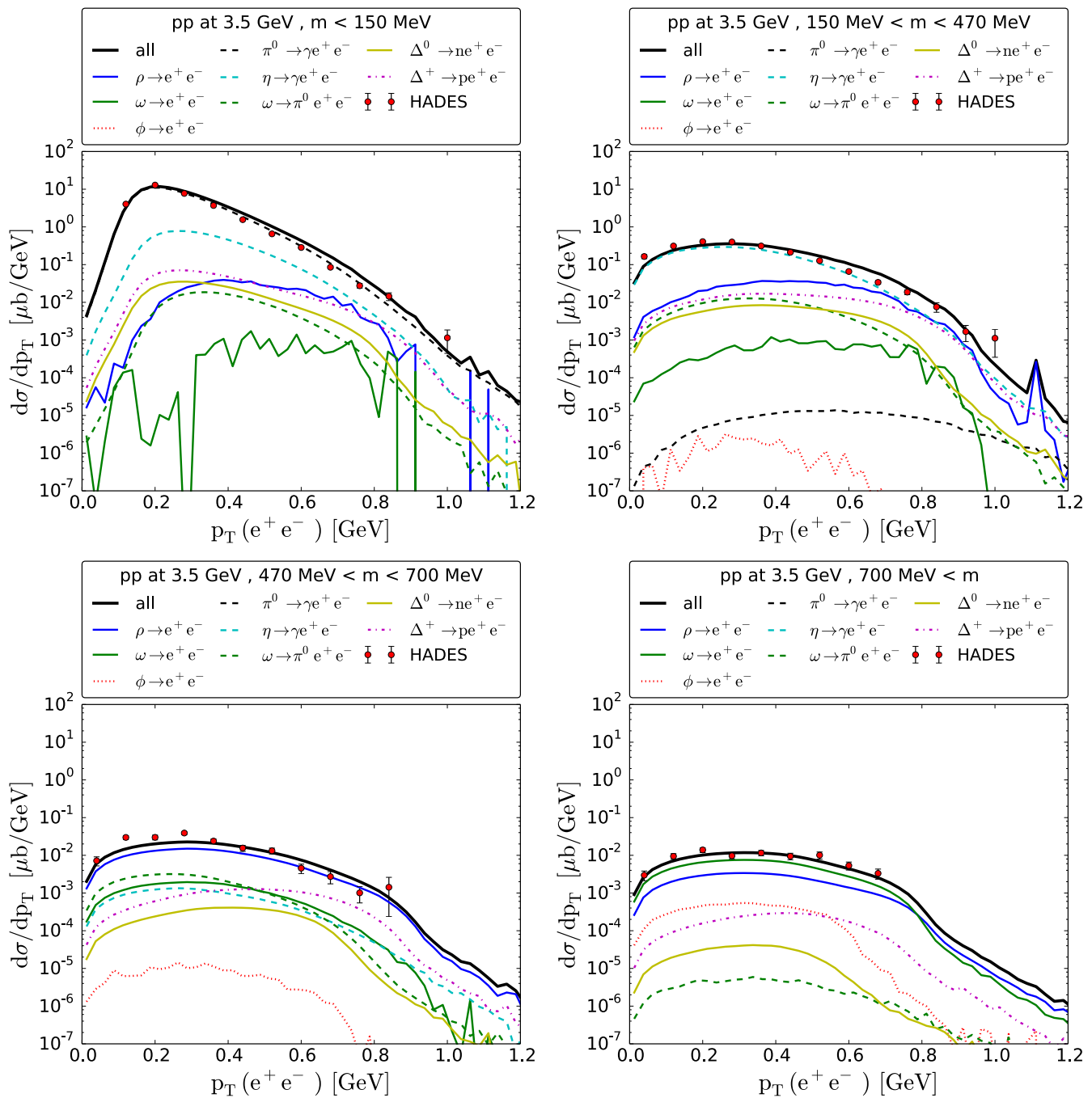

Figure 3.8: Transverse momentum spectra of dielectrons produced by pp collisions at $E_{\mathrm{Kin}}=3.5 \mathrm{GeV}$ in different invariant mass windows. Experimental data from [155]. 

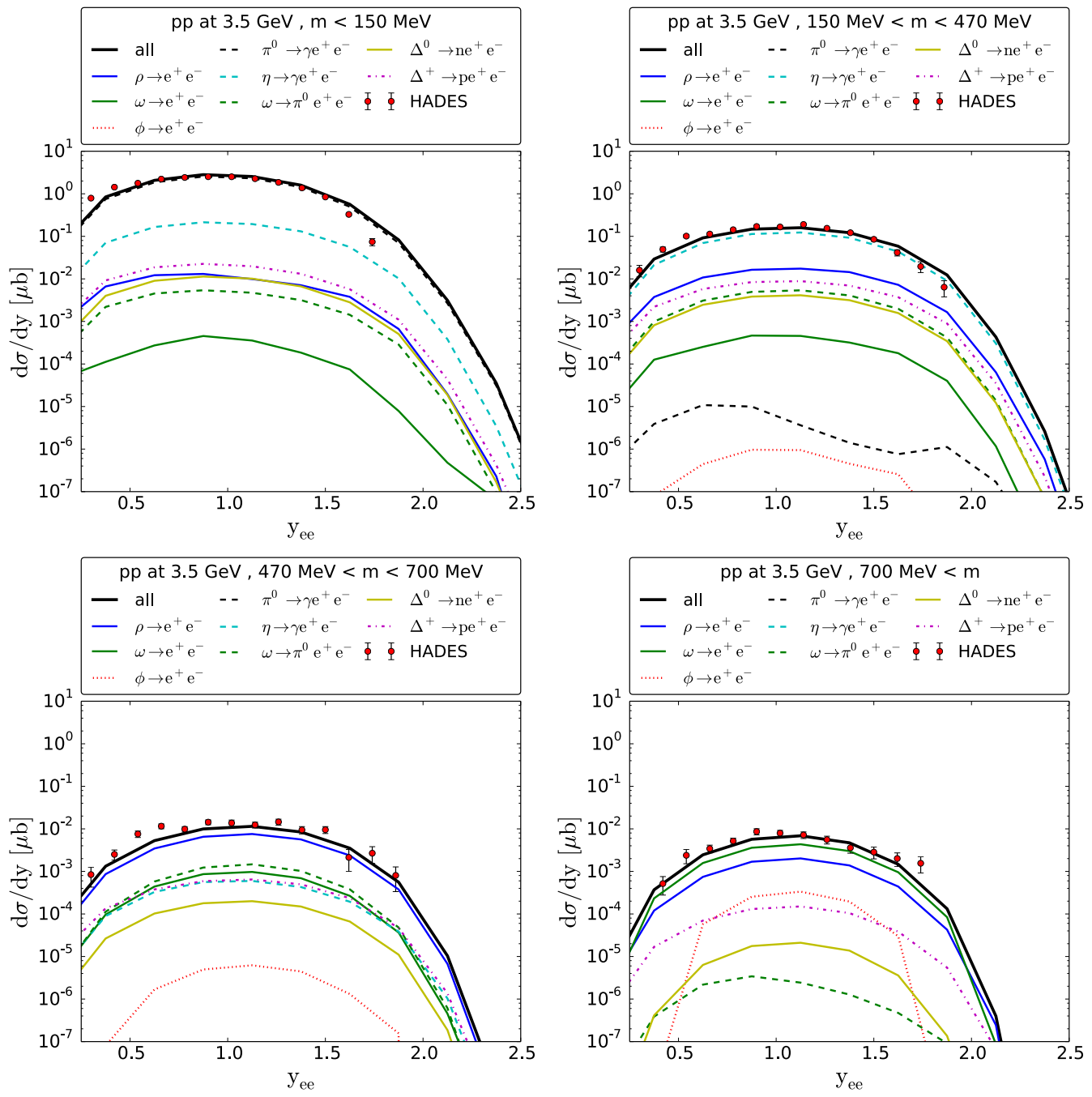

Figure 3.9: Rapidity spectra of dielectrons produced by pp collisions at $E_{\text {Kin }}=3.5 \mathrm{GeV}$ in different invariant mass windows. Experimental data from [155]. 
After discussing the general features of dilepton production in pp collisions, the focus will be specifically on the contribution from the vector mesons. The previously shown findings (Figure 3.4) for the elementary production cross-section of $\rho$ mesons measured in the hadronic channel translate directly to the corresponding dilepton spectra. At low energies $\left(E_{\text {Kin }}=1.25\right.$ and $\left.2.2 \mathrm{GeV}\right)$, where the overestimated exclusive $\rho$ production via $p p \rightarrow p p \rho$ is dominant, the $\rho$ yield is large, meaning it pushes the total yield to the upper limits of the error bars. In contrast, for the highest kinetic energy $\left(E_{\mathrm{Kin}}=3.5 \mathrm{GeV}\right)$, this is not the case. Overall, the overestimation of the exclusive cross-section does not result in an overestimation of the total dilepton yield, which indicates that the inclusive $\rho$ production is in line with the experimental dilepton results. To fully understand the dilepton production, the
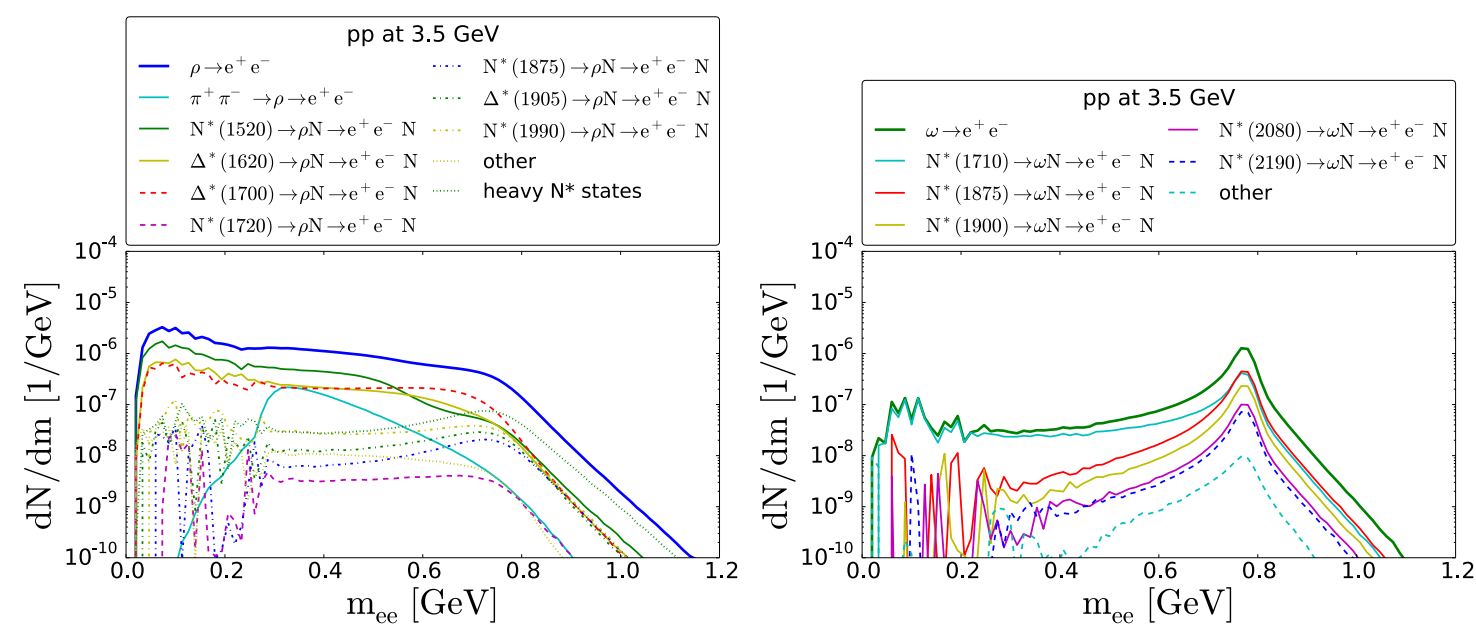

Figure 3.10: Different contributions to the invariant mass spectrum of dielectrons produced by decays of $\rho$ (left) and $\omega$ (right) mesons for pp collisions at $E_{\text {Kin }}=3.5 \mathrm{GeV}$. Heavy $\mathrm{N}^{*}$ states include contributions from $N^{*}(2080), N^{*}(2190), N^{*}(2220), N^{*}(2250)$, all of the form $N^{*} \rightarrow \rho N \rightarrow e^{+} e^{-} N$.

origin of the $\rho$ and $\omega$ resonance is investigated. Earlier studies [226, 85, 145, 255] revealed that the coupling of the vector mesons to baryonic resonances is of importance. Such information is, however, challenging to obtain in the experiment alone. Therefore only a few experimental studies are available [231]. Comparisons to theoretical models that keep track of the whole process history enable insights into important couplings by splitting the origin of the $\rho$ and $\omega$ contributions. Additionally, such studies offer the possibility to constrain resonance properties such as branching ratios. At the left of Figure 3.10 the different contributions to the overall $\rho$ dilepton yield (thick blue (upper) line, same as in Figure 3.7) are shown for pp reactions at a kinetic energy of $3.5 \mathrm{GeV}$. To allow comparisons to the overall invariant mass spectra, all dileptons in Figure 3.10 are also acceptance filtered. Two different processes are important: $\pi^{+} \pi^{-}$annihilation and the decays of different baryonic resonances. The annihilation process has a small yield as expected in elementary pp collisions because it requires rare secondary scatterings. While the $\pi \pi$ process of course has a threshold at $2 m_{\pi}$, the significant contributions below this threshold come from Dalitz-like contribution of the lighter baryonic resonances $\left(B^{*} \rightarrow \rho N \rightarrow e^{+} e^{-} N, B=N, \Delta\right)$, mainly $N^{*}(1520), \Delta^{*}(1620)$ and $\Delta^{*}(1700)$. These populate the large low-mass tail of the overall $\rho$ yield. The different shape of the $N^{*}(1520)$ is has a kinematic reason. The pole mass of the resonance is too small to produce a $\rho$ at the pole mass in the reaction $N^{*} \rightarrow N \rho$. For higher invariant masses, higher baryonic resonances are important. Especially, the combined heavy $N^{*}$ states $\left(N^{*}(2080), N^{*}(2190), N^{*}(2220), N^{*}(2250)\right)$ dominate the high 
mass tail. Other contributions include higher mesonic states and baryonic resonances that have no significant effect on the overall $\rho$ contribution. Figure 3.10 also shows the different contributions to the overall $\omega$ yield (thick green (upper) line, same as in Figure 3.7) at the right. Since the $\omega$ width is much smaller, it shows a very clear peak structure in the invariant mass spectrum; $\omega$ mesons are mainly produced by nucleon resonance decays. A clear mass ordering can be observed. The lightest baryonic resonances, $N^{*}(1710), N^{*}(1875)$, have the largest contributions followed by the heavier resonances in order of their pole masses. The contribution below the hadronic threshold that mainly forms the low-mass tail is the $N^{*}(1710)$ resonance, which is also the lightest resonance that can decay into $\omega$ with a pole mass below the $m_{N}+m_{\omega}$ threshold.

Overall the dilepton production in SMASH for pp collisions as the cleanest probe for elementary collisions is well understood and in good agreement with experimental data and offers a solid base to study larger systems.

np

The next system of interest is the elementary neutron-proton (np) system. Dilepton production has been measured by HADES, realizing np collisions by using a deuteron beam on a proton target and triggering on forward-going protons (so called spectator protons). These reactions are called quasi-free. Since the deuteron is a bound system, the nucleons inside carry additional momentum. The results for this work were obtained following an ansatz referenced in the HADES publication [153]. The neutron projectile is given additional momentum according to the momentum distribution of the PARIS potential $[267,268]$, neglecting the relatively small binding energy of the deuteron itself. Figure 3.11

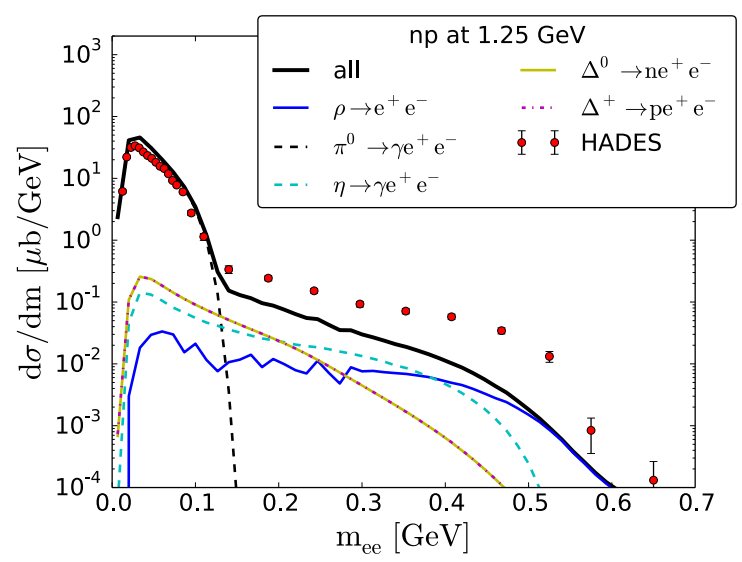

Figure 3.11: Invariant mass spectrum of dielectrons produced by quasi-free np reactions at $E_{\mathrm{Kin}}=1.25 \mathrm{GeV}$. Experimental data from [153]

shows results for the dilepton production of quasi-free $\mathrm{np}$ reactions at $E_{\mathrm{Kin}}=1.25 \mathrm{GeV}$. The additional momentum of the neutron inside the deuteron leads to a higher kinematic threshold than in the pp case (Figure 3.6) for the same energy. Hence, contributions up to $0.6 \mathrm{GeV}$ are significant. The same channels as in pp are contributing, but because the energy now reaches above the $\eta$ threshold, an additional $\eta$ yield is observable. In addition, the isospin asymmetry for $\Delta$ has vanished since both are equally likely to be excited in a primary collision. The $\pi^{0}$ contribution dominates the mass region below $0.15 \mathrm{GeV}$. In the low-mass region, $\eta, \Delta$ and $\rho$ contribute, while for masses above $0.4 \mathrm{GeV}$, the direct $\rho$ decay becomes the dominant contribution. Compared to the HADES data [153], a large 
discrepancy is observed for masses higher than $0.15 \mathrm{GeV}$, suggesting that the $\pi^{0}$ contribution is described reasonably well, but other channels are underestimated. One possible extension that would enhance the total yield includes the addition of $\mathrm{np}$ Bremsstrahlung or a $n p \rightarrow d \eta$ channel. Such extensions, however, were not successful in describing the experimental data in the similar GiBUU transport model [85]. Two other promising explanations are tested in [269] in the context of the same experimental data. The first focuses on the, in this work neglected, radiation of dileptons from an internal charged meson line and is based on a one-boson exchange model [270]. The second focuses on double $\Delta$ excitations as a possible solution [271]. Both models lead to a significantly better agreement with experimental data, with the first one slightly favored [269]. Additionally, as argued in [272], the channel $n p \rightarrow d e^{+} e^{-}$might be important for the spectrum.

Similar to other transport approaches $[85,162]$, the np system seems to be only underestimated at this low energy. The later discussed carbon-carbon collisions for example, which are close to a superposition of pp and np collisions [153], only show a similar systematic underestimation of the dilepton production around the same energy of $1 \mathrm{AGeV}$. For the higher discussed energy $\left(E_{\mathrm{Kin}}=2.0 \mathrm{AGeV}\right)$, the agreement with experimental measurements improves considerably. A reasonable agreement is also seen for higher energies in the studied proton-nucleus system. Improvements, as discussed above, are therefore left for the future.

\section{Pion beam}

Besides the discussed NN reactions, pion-beam reactions, where $\pi^{-}$scatter on a proton target, are considered. The kinetic energy of $E_{\text {Kin }}=0.56 \mathrm{GeV}$ matches upcoming HADES results [257] for this system and is specifically chosen to probe the $\rho$ production around the $N^{*}(1520)$ pole mass. Indeed, the $\rho$ dilepton decay is observed to be the dominant

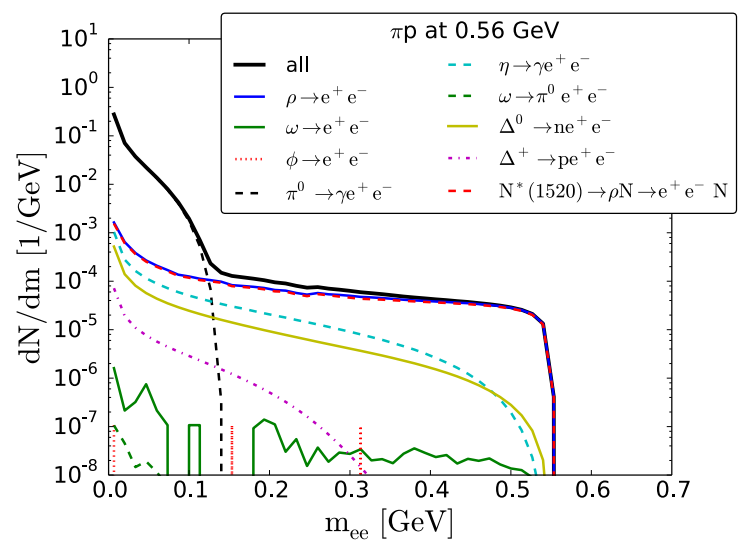

Figure 3.12: Invariant mass spectrum of dielectrons produced by $\pi \mathrm{p}$ reactions at $E_{\text {Kin }}=$ $0.56 \mathrm{GeV}$.

contribution to the dilepton invariant mass spectrum from $\pi \mathrm{p}$ at $E_{\mathrm{Kin}}=0.56 \mathrm{GeV}$ (Figure 3.12). Only for invariant masses lower than $0.15 \mathrm{GeV}$, it is exceeded by the $\pi$ decay contribution. Other smaller contributions include $\eta$ and $\Delta^{0}$, negligible are $\Delta^{+}, \omega$ and $\phi$. Compared to pp the ordering of the $\Delta^{+}$and $\Delta^{0}$ is inverted for this system due to the same reason as mentioned before: because of charge conservation, only $\Delta^{0}$ can be produced in primary collisions. The sharp kinematic threshold at $0.56 \mathrm{GeV}$ due to the available center of mass-energy is noticeable as well. The red dashed line in Figure 3.12 
indicates the $N^{*}(1520)$ contribution to the $\rho$ spectrum. It is the only relevant contribution to the $\rho$ spectrum and with this to the overall spectrum above $0.15 \mathrm{GeV}$. Therefore this setup provides a good opportunity to test and constrain the coupling of the $\rho$ to the $N^{*}(1520)$. Currently, SMASH treats the $N^{*}(1520)$ Dalitz decay $\left(N^{*}(1520) \rightarrow e^{+} e^{-} N\right)$ via the strict Vector Meson Dominance assumption, where the resonance decays via an intermediate $\rho$ meson. The pion beam experimental data will be valuable to constrain this assumption and the extensive theoretical investigation of the $\rho-N$ interaction in general, which started with $[273,274,275]$ (see [276] for a review). For example, a test of the simple "QED point-like" $R \gamma^{*}$ model [277], where the Dalitz decay is performed directly and the involved electromagnetic form factor is chosen to be constant, would be possible.

\section{Proton-nucleus collisions}

The dilepton production in proton-nucleus (pA) collisions, as a cold nuclear matter scenario, is discussed for a proton projectile that scatters on a niobium target with $E_{\text {Kin }}=$ $3.5 \mathrm{GeV}(\mathrm{pNb})$. To obtain the cross-section the value reported in [156] $\left(\sigma_{\mathrm{pNb}}=848 \pm 127\right.$ $\mathrm{mb}$ ) is used. A comparison to HADES data [156] of the invariant electron-pair mass spec-

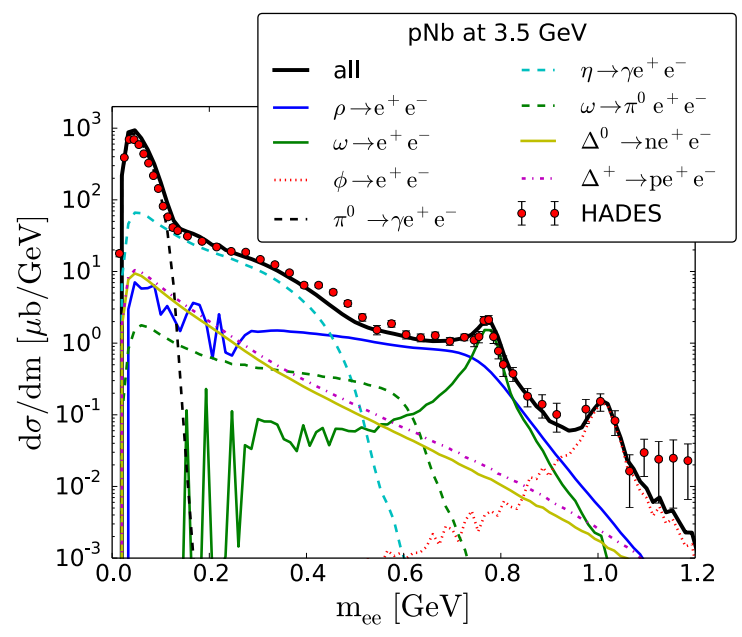

Figure 3.13: Invariant mass spectrum of dielectrons produced by pNb reactions at $E_{\text {Kin }}=$ $3.5 \mathrm{GeV}$. Experimental data from [156].

trum is displayed in Figure 3.13. In the low-mass region, the $\pi$ and $\eta$ contributions are prominent. The $\pi$ peak is slightly overestimated, hinting at a problem with the overall normalization. The experimental data also reveal a stronger shoulder around $0.5 \mathrm{GeV}$. Overall experimental data and SMASH results are in reasonable agreement. Therefore, it seems that the resonance description based on vacuum properties can account for the dynamics to some extent. In contrast, the underestimation around $0.5 \mathrm{GeV}$ might hint at an onset of a broadening of the $\rho$-like contribution due to a stronger coupling of the $\rho$ to baryonic resonances.

Figure 3.14 displays the invariant mass spectrum for two different dilepton momentum windows $\left(0<\mathrm{p}_{\mathrm{ee}}<800 \mathrm{MeV}\right.$ and $\left.\mathrm{p}_{\mathrm{ee}}>800 \mathrm{MeV}\right)$. Again, an overall reasonable agreement is observed. The underestimation of the shoulder at $0.5 \mathrm{GeV}$ is only seen for the low momentum dileptons, which further points to a broadening of the $\rho$ spectral shape. Effects by the medium are enhanced for the low momentum dileptons since the decaying resonances are traversing the medium longer. The $\omega$ and the $\phi$ peak are nicely matched. On the one hand, this validates the extracted $N^{*} \rightarrow \phi N$ branching ratio. If one compares 

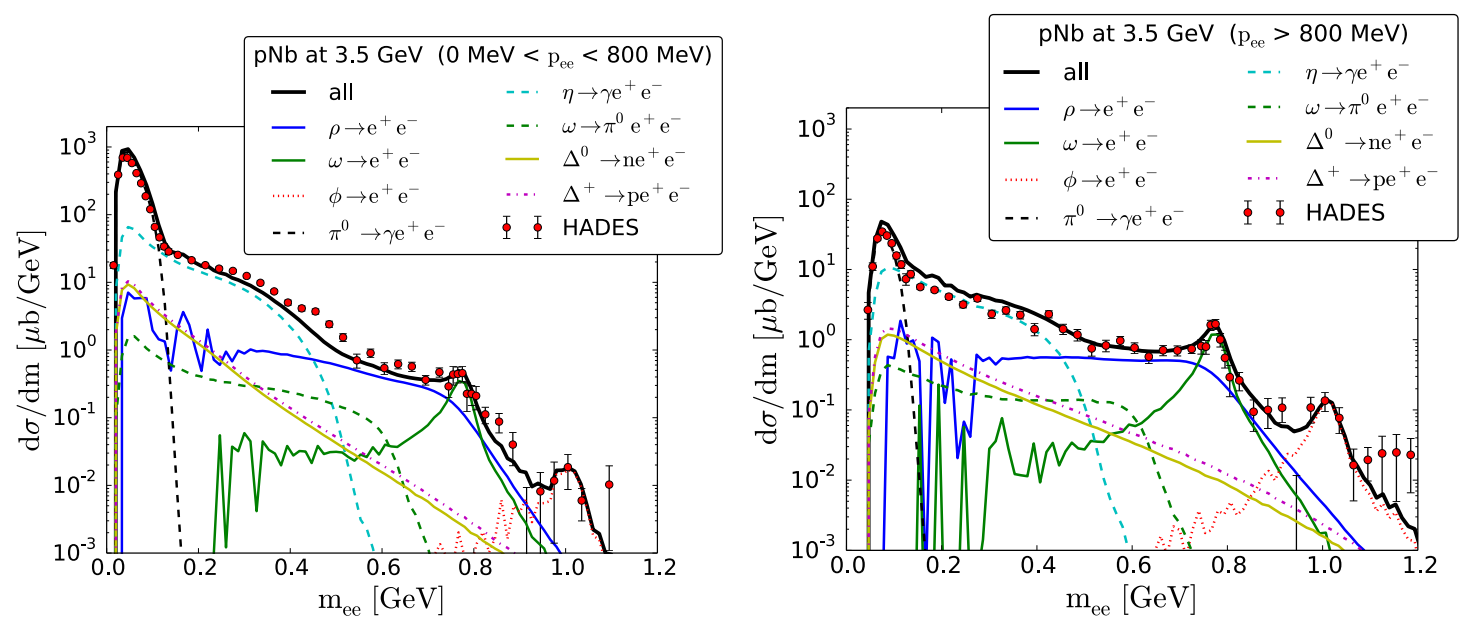

Figure 3.14: Invariant mass spectrum of dielectrons produced by pNb reactions at $E_{\text {Kin }}=$ $3.5 \mathrm{GeV}$ in different dielectron momentum $\left(\mathrm{p}_{\mathrm{ee}}\right)$ windows. Experimental data from [156].

the peaks between momentum cuts, the peaks are suppressed for the low momentum dileptons. This is caused by the absorption of low-momentum resonances inside the cold nuclear matter. These findings support the results reported in [156]. Furthermore, this validates the microscopic dynamics since absorption is a medium effect intrinsic to transport approaches (in contrast to modification of spectral shapes). Initial pp and $\mathrm{np}$ reactions are roughly equally contributing to the pA yield. The relatively solid description of the experimental data, therefore, hints that the dilepton production cross-section in np collision at this energy is in better agreement with experimental results than for the lower energies (cf. Figure 3.11).

The reported findings for the proton-induced reactions (including the results for pp and np) align with the GiBUU approach [85]. Differences are found in the low-mass $\omega \rightarrow e^{+} e^{-}$ contributions and the $\rho \rightarrow e^{+} e^{-}$yield composition. The latter is caused by the adaptation of recent PDG branchings and, in general, a different set of resonance states. Additionally, the discussion here is extended to include the spectra for the two pee windows. As already mentioned, similar efforts as in [85] to improve the agreement with the np experimental data, such as Bremsstrahlung, are left for the future.

\section{Nucleus-nucleus collisions}

In the following, results for four larger nucleus-nucleus collision systems are shown and compared to experimental data, wherever available. This section also addresses which spectra are sensitive to additional medium modifications by deviating from results based on vacuum properties.

\section{$\mathrm{CC}$}

Light nucleus-nucleus collisions offer a good starting point for studying dilepton production under the assumption of vacuum resonance properties in larger collision systems. Results in this section include invariant mass spectra of the produced dielectrons in carbon-carbon (CC) collisions for two kinetic energies: $E_{\text {Kin }}=1.0 A \mathrm{GeV}$ and $E_{\text {Kin }}=2.0 \mathrm{AGeV}$.

Figure 3.15 shows the spectrum for the lower beam energy on the left with main contributions originating from the $\pi, \Delta, \eta$ and $\rho$ channels. $\omega$ decays do not have a significant impact 

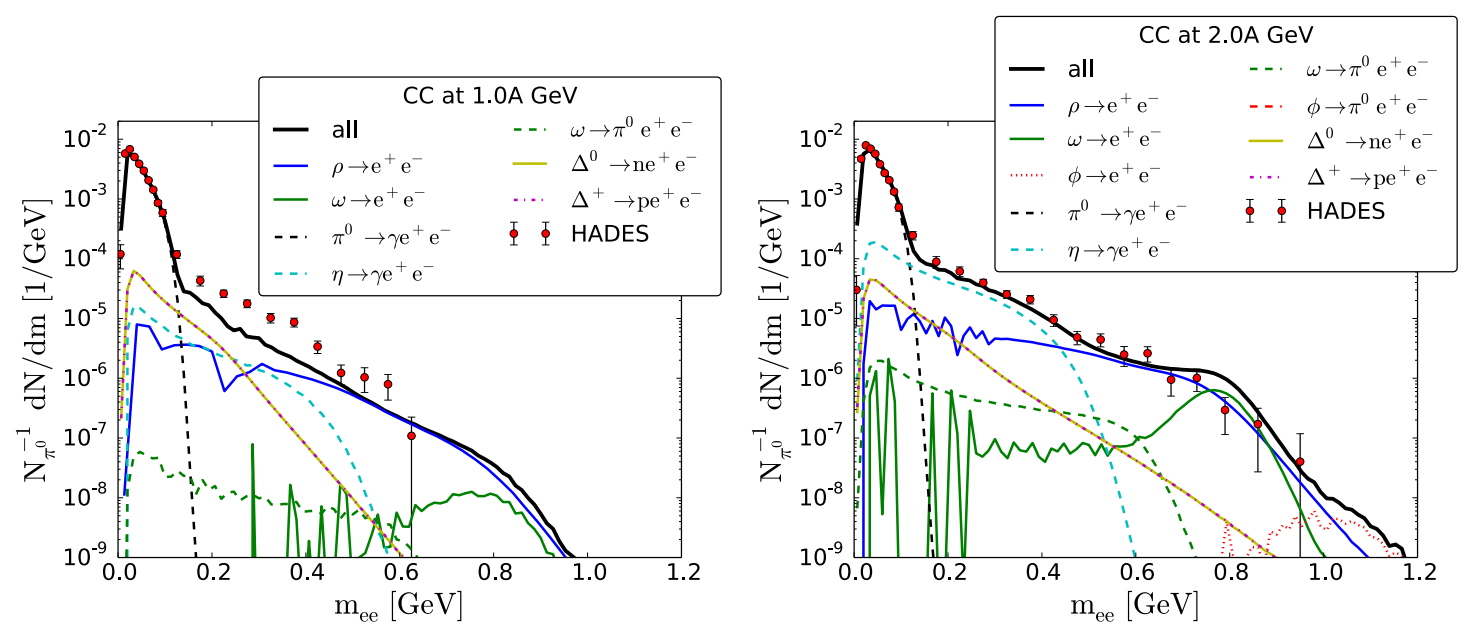

Figure 3.15: Invariant mass spectrum of dielectrons produced by $\mathrm{CC}$ reactions. Left: $E_{\text {Kin }}=1.0 A \mathrm{GeV}$. Right: $E_{\text {Kin }}=2.0 A \mathrm{GeV}$. Data from $[157,278]$.

on the overall yield. The $\Delta^{0}$ and $\Delta^{+}$yields are compatible due to the equal initial numbers of protons and neutrons, and therefore similar production probability. Comparing with HADES data [157] reveals a disagreement in the low mass region between $0.15 \mathrm{GeV}$ and $0.4 \mathrm{GeV}$. Even though the shape potentially matches the data, the total yield is underestimated. This can be understood by recalling the previously discussed elementary results for $\mathrm{pp}$ and $\mathrm{np}$. The dilepton production in pp collisions is in good agreement with data, but $\mathrm{np}$ collisions produce too few dileptons. In consequence, an underestimation around the same kinetic energy is expected since CC is known to be close to a mere superposition of binary NN reactions [153]. The results for the dilepton invariant mass spectrum in CC collisions at the higher energy of $E_{\mathrm{Kin}}=2.0 \mathrm{AGeV}$ are shown in Figure 3.15 on the right. The $\pi$ and $\eta$ contributions dominate the spectrum up to $0.4 \mathrm{GeV}$, while above this mass, the yield mainly consists of $\rho$ and $\omega$ contributions. At the highest masses, the $\phi$ peak is broadened due to the low resolution of the detector. The data [158] is nicely described by the total yield. In the region around the $\omega$-pole mass $\left(M_{0, \omega}=0.783 \mathrm{GeV}\right)$, the $\rho$ and $\omega$ contributions are slightly overestimated, which might already indicate an onset of inmedium modifications. Following the same argumentation as for $E_{\mathrm{Kin}}=1.0 \mathrm{AGeV}$, that $\mathrm{CC}$ equals a NN superposition as reported in [153], the agreement with the experimental spectrum suggests that the dilepton emission for elementary np collisions for energies higher than $E_{\mathrm{Kin}}=1.0 \mathrm{GeV}$ is in better agreement with experimental data.

Compared to the most recent results from the similar UrQMD transport approach for the same system ([115], Figure 1), the results presented here compare overall similarly to data, but are in better agreement in the low and the vector meson pole-mass region. A similar or better agreement than UrQMD is also found for other systems[115, 163]. On the one hand, differences originate in the detailed investigation of the $\rho$-like contribution by studying the decay of baryonic resonances (Figure 3.10). The input of the relevant branching ratios in SMASH was carefully constrained by the dilepton data and more recent PDG data [243]. On the other hand, the different thresholds of the vector meson contributions of the $\rho$ and the $\omega$ lead to notable differences. In particular, for the discussed CC system, the $\rho$ is, as the second-largest yield, a significant contribution in the low-mass region. The $\omega$ low-mass tail is not important for the overall yield. The relative difference to the $\omega$ Dalitz decay is, however, smaller than for pp. In principle, the $\phi$ meson again has low-mass contributions, 
but its yield is again too small to be visible on the chosen scale.

The fact that the description of the dilepton production with SMASH matches the data for $E_{\text {Kin }}=2.0 A \mathrm{GeV}$ validates the resonance treatment and the approach for this energy. It also shows that no in-medium modifications seem necessary to describe the dilepton production for such small systems or at least that invariant mass dilepton data are not sensitive to such modifications. However, it is important to mention that transport approaches and the shown spectra do include collisional broadening, even if the description of the resonances is still based on vacuum properties.

\section{$\mathrm{ArKCl}$}

On the basis of the dilepton production in elementary and small nucleus-nucleus systems shown above, larger systems are explored. A good example for an intermediate-sized collision system is the $\mathrm{ArKCl}$ system at $E_{\mathrm{Kin}}=1.76 \mathrm{AGeV}$ measured by HADES [159]. Within SMASH it is modeled with a ${ }^{40} \mathrm{Ar}$ projectile hitting a ${ }^{37} \mathrm{Ar}$ nucleus target to emulate an average of the ${ }^{35} \mathrm{Cl}$ and ${ }^{39} \mathrm{~K}$ composition. Like most of the discussed dielectron invariant

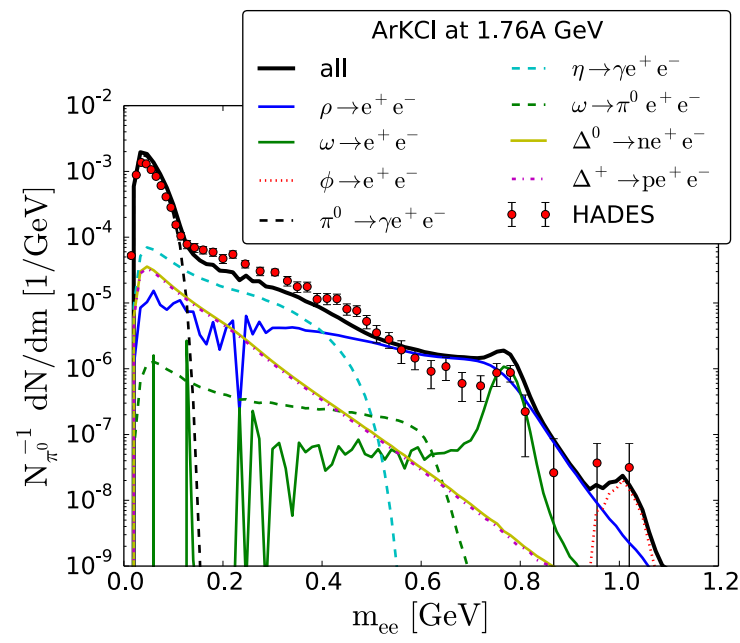

Figure 3.16: Invariant mass spectrum of dielectrons produced by $\mathrm{ArKCl}$ collisions at $E_{\mathrm{Kin}}=1.76 \mathrm{~A} \mathrm{GeV}$. Experimental data from [159].

mass spectra, the ArKCl yield shown in Figure 3.16 is dominated by $\pi$ and $\eta$ in the low and the vector mesons $(\rho, \omega, \phi)$ in the higher invariant mass region above $0.5 \mathrm{GeV}$. Since there are more neutrons than protons in the colliding nuclei, the $\Delta^{0}$ is slightly above the $\Delta^{+}$yield. Although the spectrum is in reasonable agreement with the experimental data [159] for low and highest invariant masses, two distinct issues are revealed compared to experimental data. First, the $\rho$ contribution is too large in the region between $0.6 \mathrm{GeV}$ and $0.8 \mathrm{GeV}$. This might be connected to the overproduction in the exclusive $\rho$ cross-section discussed in Section 3.1.1. The $\rho$ in pp reactions at $E_{\text {Kin }}=1.76 A \mathrm{GeV}\left(\sqrt{s_{N N}}=2.61 \mathrm{GeV}\right)$ is almost solely produced by the overestimated exclusive process $p p \rightarrow p p \rho$ (Figure 3.4). The second issue is an underestimation in the mass region between $0.15 \mathrm{GeV}$ and $0.5 \mathrm{GeV}$. In combination, both issues indicate the limit of the assumption of resonances with vacuum properties. The low mass region is known to be enhanced by in-medium modifications, i.e. a broadening of the vector meson spectral functions, in particular of the $\rho$ meson [136]. This broadening also has an influence on the $\rho$ pole mass region since a broadening will decrease the yield in this region. That in-medium modifications are relevant is also 
supported by the agreement with experimental data for CC reactions at the similar energy of $E_{\mathrm{Kin}}=2.0 \mathrm{AGeV}$. The agreement is expected to translate to a larger system if no additional medium effects become relevant.

Therefore, this result suggests that dilepton emission in systems as large as ArKCl is sensitive to in-medium modifications of resonances that go beyond the intrinsic collisional broadening mentioned above. To verify this hypothesis, a comparison with a coarsegraining approach [117] that employs in-medium modifications of spectral functions is presented in Section 3.1.3.

$\operatorname{AgAg}$
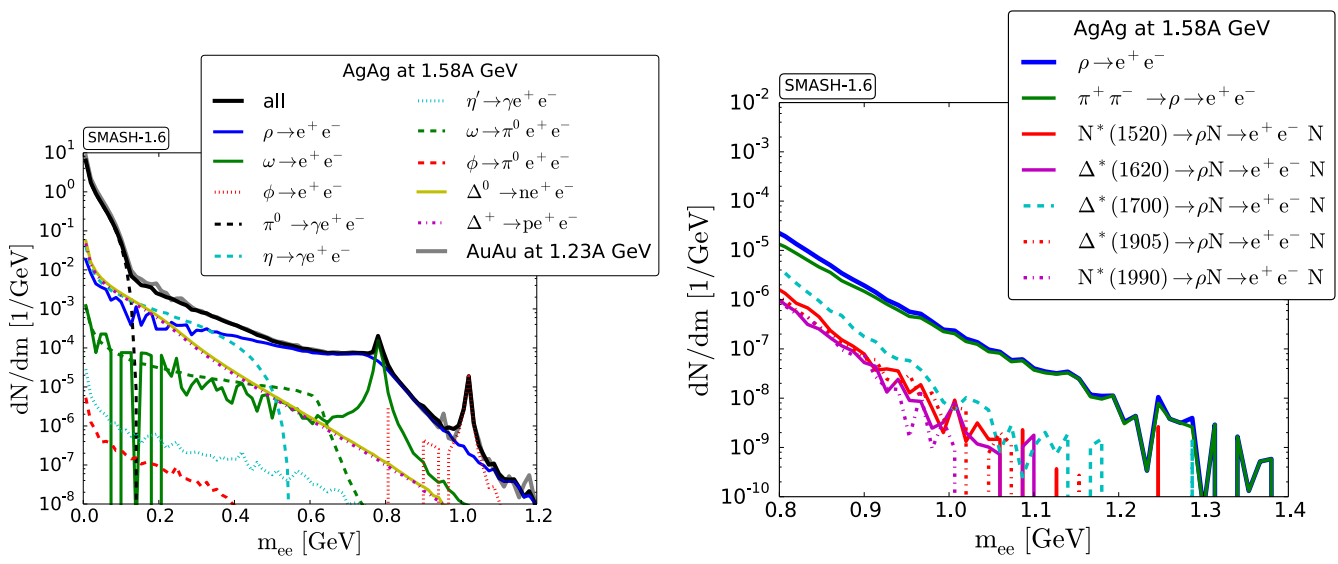

Figure 3.17: Left: Invariant mass spectrum of dielectrons produced by $\mathrm{AgAg}$ collisions at $E_{\mathrm{Kin}}=1.58 \mathrm{AGeV}$. Right: Origins to the $\rho \rightarrow e^{+} e^{-}$decay contribution in the high invariant mass region.

The comparison between the different collision systems allows assessing the magnitude of medium effects. Figure 3.17 (left plot) shows the invariant mass spectrum for dielectrons produced in $\mathrm{AgAg}$ collisions for a kinetic energy of $1.58 \mathrm{~A} \mathrm{GeV}$. The different channels contributing to the spectrum are displayed as well, with dominant contributions from the $\pi^{0}$ decay for low masses and the vector meson decays $(\rho, \omega$ and $\phi)$ around their respective pole masses. Also shown in Figure 3.17 (left plot, gray line) is the yield of the other large collision system studied by HADES: $\mathrm{AuAu}$ at $E_{\mathrm{Kin}}=1.23 \mathrm{AGeV}$, which is discussed in the next section. The total yield of the larger AuAu system with lower beam energy is strikingly similar. Only a slightly higher yield is observed for smaller masses. The higher beam energy of $\mathrm{AgAg}$ seems to overall compensate for the smaller system concerning the dilepton production.

Of special interest in the invariant mass spectrum is the yield above the $\phi$ peak, which offers insights into the temperature of the medium and might become experimentally accessible for the first time with the upcoming high-statistics data for AgAg at these low energies. Therefore, it is crucial to understand all dilepton emitting sources in this region of the spectrum. As seen in Figure 3.17 (left plot), the dilepton production observed with SMASH above the $\phi$ peak is dominated by the $\rho$ contribution. Figure 3.17 (right plot) therefore shows the different processes from which the $\rho$ yield originates in the mass region above and around the $\phi$ pole mass. The $\rho$ contribution is either produced by the decay of baryonic resonances into $\rho N$ or by $\pi$ annihilation. Observed is a dominance of the $\pi$ annihilations, especially in the mass region above the $\phi$ peak. Sub-leading contributions to 
the $\rho$ tail are found for different $\Delta^{*}$ and $N^{*}$ decays. Most prominent here is the $\Delta^{*}(1700)$.

\section{$\mathrm{AuAu}$}

The largest collision system discussed in this work is gold-gold ( $\mathrm{AuAu}$ ) scattering at $E_{\mathrm{Kin}}=$ $1.23 \mathrm{~A} \mathrm{GeV}$, matching the measurements by HADES [258]. The invariant mass dielectron

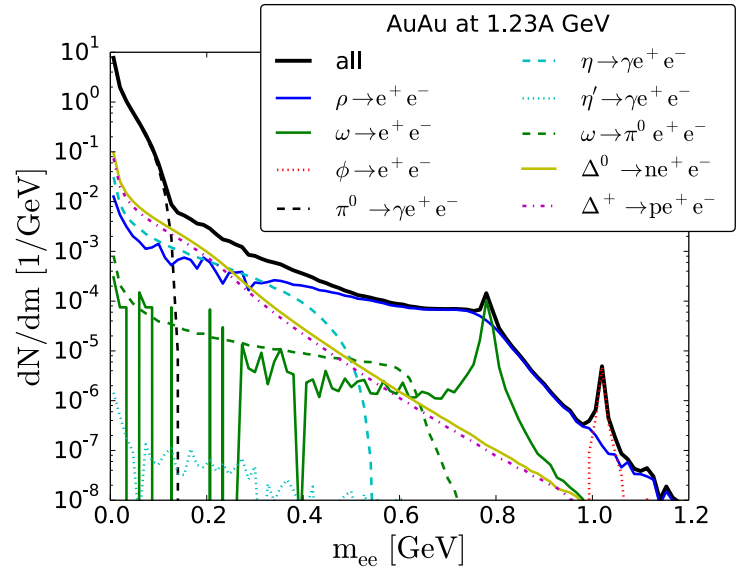

Figure 3.18: Invariant mass spectrum of dielectrons produced by $\mathrm{AuAu}$ collisions at $E_{\text {Kin }}=1.23 A \mathrm{GeV}$.

spectrum (Figure 3.18), which is not acceptance filtered, reveals some differences to the previous cases even without a comparison to the not yet available experimental data. The $\Delta$ yield is larger in relation to other contributions than for smaller systems. The $\phi$ peak is most prominent in this spectrum and the $\rho$ contribution shows a slight bump at the pole mass since the reaction $\pi \pi \rightarrow \rho$ dominates over the different Dalitz-like $N^{*}$ and $\Delta^{*}$ contributions. The large amount of secondary reactions can explain both effects. Figure 3.18 also shows an $\eta^{\prime}$ contribution, which is only visible since a large vertical scale is chosen for this plot. This illustrates that its contribution is negligible, especially for smaller systems. Furthermore, the limited statistics suggest that $\eta^{\prime}$ is rarely produced even in large systems. The $\rho$ is dominant up to the $2 m_{\pi}$ threshold and remains one of the leading contributions for lower masses. Even though the statistics are limited below the hadronic threshold, it can be seen that the direct $\omega$ contribution is on the same order of magnitude as the $\omega$ Dalitz contribution. This shows that towards larger systems (compare Figure 3.7 and Figure 3.15, right plot), the difference between the direct and Dalitz $\omega$ decay becomes smaller. In other words, the sub-threshold contributions become more prominent the larger the system. Only the $\phi$ contribution remains small since it is suppressed for the low energies discussed here. This might change for higher energies with a larger overall $\phi$ production.

A first partial comparison of the dilepton emission in $\mathrm{AuAu}$ systems with transverse data is reported in Figure 3.23. A complete set of more differential predictions is given in Appendix B, which are also used to study the dependence of medium effects on invariant mass, transverse momentum, rapidity and centrality in the following section.

\subsubsection{Coarse-graining results}

To investigate the effect of in-medium modifications, the hadronic evolution of SMASH is coarse-grained (CG) in this section following the original idea from [114]. This means that 
macroscopic quantities are extracted locally from the microscopic transport model, enabling the determination of thermal dilepton emission from those regions. The framework used here is developed by Endres et al. [117] and introduced in Section 2.4.3. To recall, the dilepton radiation is a mix of the dilepton production from thermal dilepton emission rates and the usual hadronic transport contributions. Thermal rates together with the freeze-out contributions result in the coarse-graining contributions for the $\rho$ and $\omega$ (CG- $\rho$ and CG- $\omega$ ). The cocktail is complemented with the relevant transport contributions of $\pi$, $\eta$ and $\phi$ from SMASH.

Before looking at the results for dilepton emission in the context of experimental data, the thermodynamic properties of the different systems are considered. The evolution of macroscopic quantities in the central cell at the origin of the coarse-graining grid for $\mathrm{ArKCl}, \mathrm{AgAg}$ and $\mathrm{AuAu}$, at their SIS energies is given in Figure 3.19. The upper plot shows the evolution of the baryon $\rho_{B}$ and energy $\epsilon$ density in units of the ground-state density, the bottom plot the extracted temperature and baryon chemical potential for the three systems. The figure illustrates the differences in the system's evolution at the center of the collision: $\mathrm{ArKCl}$ as the smallest system only builds up a smaller density than the larger systems. $\mathrm{AgAg}$ builds up the density quicker since the beam energy is higher and falls off faster than AuAu. Both systems, however, behave similarly in terms of the maximum density that they reach. AuAu, as the largest system, maintains large densities the longest. The differences in density mainly translate into differences in the decline of $T$ and $\mu_{B}$ over time, which shows a clear ordering with the system size. All systems reach a plateau in baryon chemical potential at $750-900 \mathrm{MeV}$ and a similar maximum temperature of around $100 \mathrm{MeV}$. Note that the values shown here are maximum values at the point of highest density (central cell) in the system, which illustrates the phase diagram's maximal reach. The result confirms that the cell evolution is reasonable since the expectation and results reported in $[117,279]$, that are based on the hadronic space-time evolution of UrQMD, are matched. This further validates the SMASH approach and forms the basis for the more advanced analysis of the dilepton emission of the coarse-grained evolution.

Results for the three largest systems studied here are presented - ArKCl, AgAg and AuAu. The two different approaches used in this work are compared: First, the dilepton yield from the transport model SMASH as discussed above (referred to as non-CG); although the medium effect of collisional broadening is included, no in-medium modifications are incorporated. Second, the outcome from the coarse-graining approach, which employs thermal rates including an in-medium description for the $\rho$ and $\omega$ meson; those mediummodified dilepton contributions are combined with unmodified cocktail contributions $(\pi$, $\eta, \phi)$ from the SMASH simulations.

\section{Results for the dilepton emission}

First, the focus is on the dilepton emission from $\mathrm{ArKCl}$ collisions at $E_{\mathrm{Kin}}=1.76 \mathrm{AGeV}$. The total SMASH vacuum transport result $($ non- $C G)$ is underestimating the invariant mass spectrum for this system in the low mass region and overestimating it in the $\rho$ pole mass region (cf. Figure 3.16). Figure 3.20 shows the results from the coarse-graining approach, which again are cut in momentum and angular distribution as well as filtered for the acceptance [266] to compare to experimental data from HADES [159]. All solid lines refer to SMASH dilepton production (the same as in Figure 3.16). Only the $\pi$ and $\phi$ yields are important for the overall spectrum at low and high invariant masses, respectively. The dashed contributions for $\rho$ and $\omega$ display results from the coarse-graining approach and include the thermal dilepton rates containing in-medium modifications and the freeze-out 

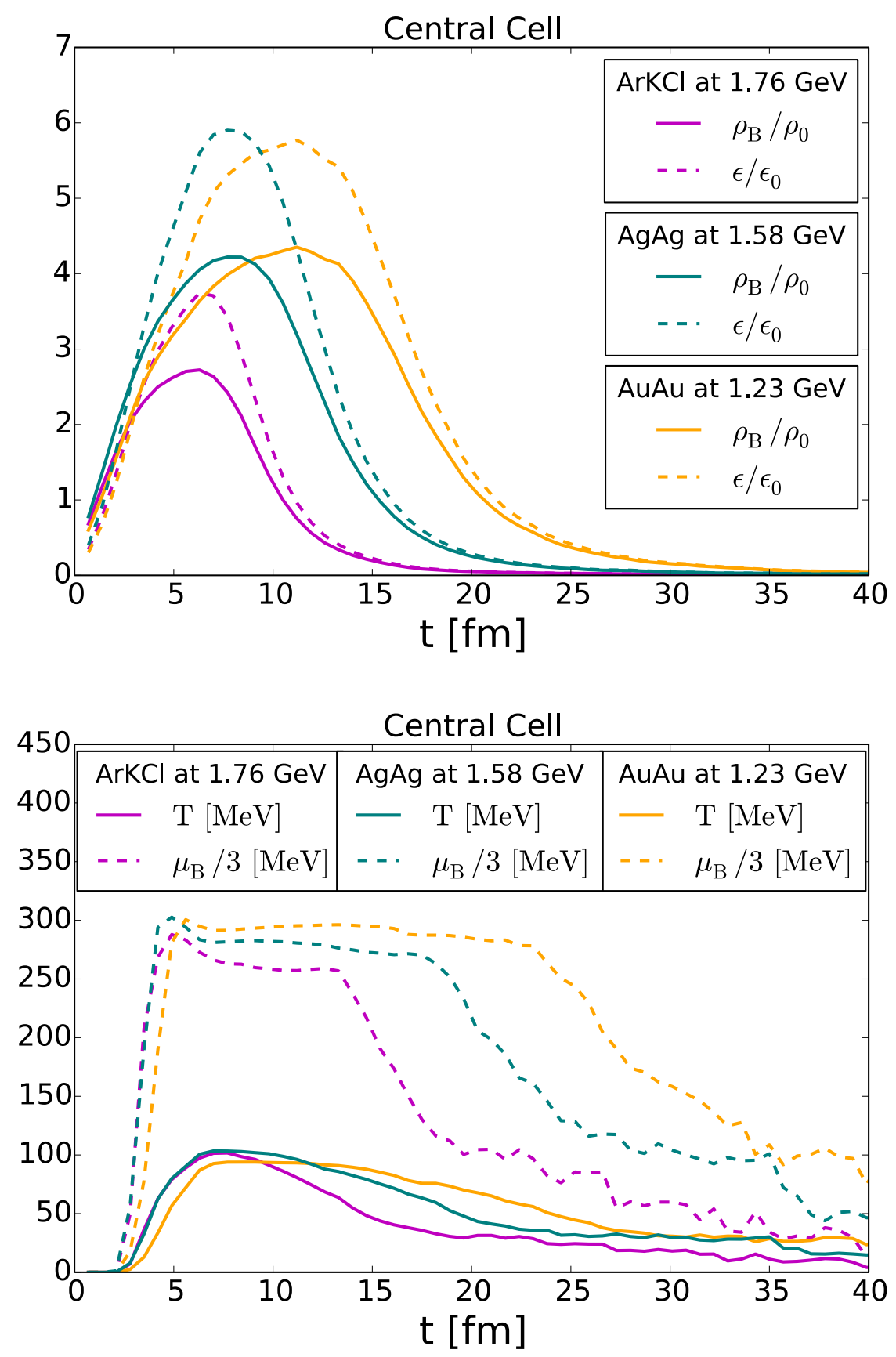

Figure 3.19: Evolution of the energy and baryon density in units of the ground-state densities $\epsilon_{0}=146.5 \mathrm{MeV} / \mathrm{fm}^{3}$ and $\rho_{0}=0.16 \mathrm{fm}^{-3}$ (upper plot), temperature $T$ and baryochemical potential $\mu_{B}$ (lower plot) in the most central cell over time for ArKCl collisions at $E_{\mathrm{Kin}}=1.76 \mathrm{AGeV}, \mathrm{AgAg}$ collisions at $E_{\mathrm{Kin}}=1.58 \mathrm{AGeV}$ and $\mathrm{AuAu}$ collisions at $E_{\mathrm{Kin}}=1.23 A \mathrm{GeV}$. All results for SMASH-1.6 [211]. 


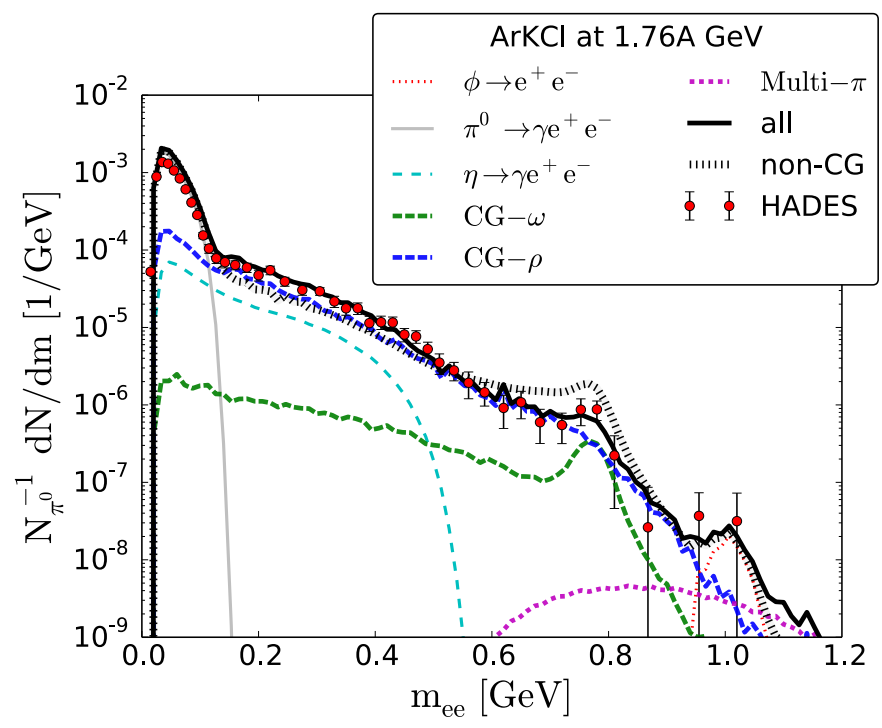

Figure 3.20: Invariant mass spectrum of dielectrons produced by $\mathrm{ArKCl}$ collisions at $E_{\text {Kin }}=1.76 A \mathrm{GeV}$ within the Coarse-Graining approach. Dashed lines from coarsegraining and solid lines from SMASH dilepton production (as in Figure 3.16). Experimental data from [159].

contributions for cold cells. Also, the multi- $\pi$ contribution is added but has little effect on the overall spectrum due to the low beam energy. On the contrary, the effects on the vector meson dilepton yield are large. For both $\rho$ and $\omega$, the yield is shifted from the pole mass to the low-mass region.

Quantitatively, the agreement of the overall yield (all) with experimental data [159] in Figure 3.20 is greatly improved with the in-medium modifications of the vector meson spectral functions employed in the coarse-graining approach compared to the SMASH dilepton production based on vacuum resonance properties. Only the normalization on the $\pi$ multiplicity leads to an overestimation of the $\pi$ peak for low invariant masses and, consequently, a slight overproduction around $0.15 \mathrm{GeV}$. It can be concluded that a sensitivity to in-medium modifications in the $\mathrm{ArKCl}$ spectrum is confirmed.

The coarse-graining results for the dilepton production in $\mathrm{AgAg}$ reactions with $E_{\mathrm{Kin}}=$ $1.58 \mathrm{~A} \mathrm{GeV}$ are seen in Figure 3.21. The result in the figure reveals that the multi- $\pi$ yield becomes dominant beyond the $\phi$ peak. This essentially is the same finding when only considering the dilepton production from the SMASH approach (cf. Figure 3.17), where the (two- $\pi) \rho$ state is the largest contribution: the region above the $\phi$ pole mass is dominated by $\pi$ annihilation reactions. If the total dilepton production from the transport approach alone (non-CG in Figure 3.21) is compared to the coarse-graining yield, a clear difference is seen. The yield is shifted away from the vector meson peaks. This finding confirms the result in ArKCl. The dilepton production in larger systems is sensitive to including medium modifications to the vector mesons spectral function. Comparing the total contributions from the coarse-graining approach already to the below discussed $\mathrm{AuAu}$ (yellow line in Figure 3.21) reveals again (cf. Figure 3.17) that both systems emit dileptons similarly. Only the AuAu spectrum in the $\rho$ dominated region between 0.15 and $0.6 \mathrm{GeV}$ shows a small but consistent higher emission than AgAg, which grows towards smaller invariant masses and hints at larger medium effects in the larger ( $\mathrm{AuAu})$ system. 


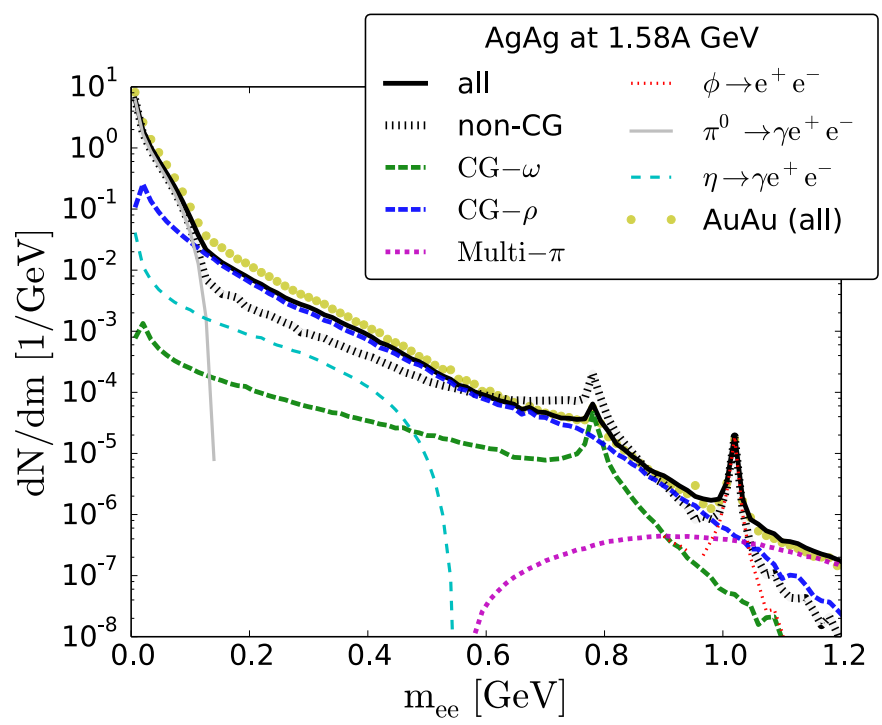

Figure 3.21: Invariant mass spectrum of dielectrons produced by $\mathrm{AgAg}$ collisions at $E_{\text {Kin }}=1.58 A \mathrm{GeV}$ within the coarse-graining approach. Dashed lines from coarse-graining and solid lines from SMASH dilepton production (as in Figure 3.17).

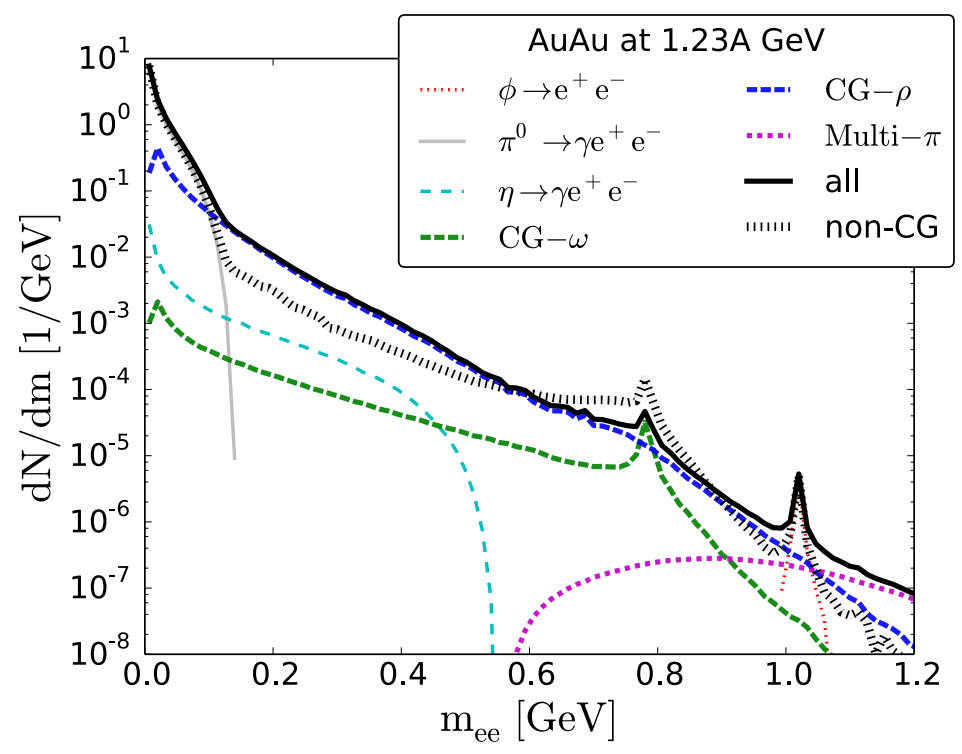

Figure 3.22: Invariant mass spectrum of dielectrons produced by $\mathrm{AuAu}$ collisions at $E_{\text {Kin }}=1.23 \mathrm{~A} \mathrm{GeV}$ within the Coarse-Graining approach. Dashed lines from coarsegraining and solid lines from SMASH dilepton production (as in Figure 3.18). 
Predictions for $\mathrm{AuAu}$ collisions at $E_{\mathrm{Kin}}=1.23 \mathrm{~A} \mathrm{GeV}$ within the coarse-graining approach, which are not acceptance filtered, are shown in Figure 3.22. The comparison of the SMASH dilepton result for the total yield from Figure 3.18 (non- $C G$ ) with the spectrum from the coarse-graining approach $(a l l)$ hints at a large modification of the yield. Differences are observed in the low-mass region and around vector meson pole masses analogously to $\mathrm{ArKCl}$ and $\mathrm{AgAg}$. Still, the effect in the intermediate-mass region seems more pronounced than in ArKCl. Also, in the intermediate invariant mass region, the multi- $\pi$ contribution leads to deviations, similar to AgAg. Again the only relevant yields from the SMASH contributions are the $\pi$ and $\phi$ channels.

The presented findings for $\mathrm{ArKCl}, \mathrm{AgAg}$ and $\mathrm{AuAu}$ overall align nicely with previous results obtained with the UrQMD coarse-graining transport approaches [117, 279].

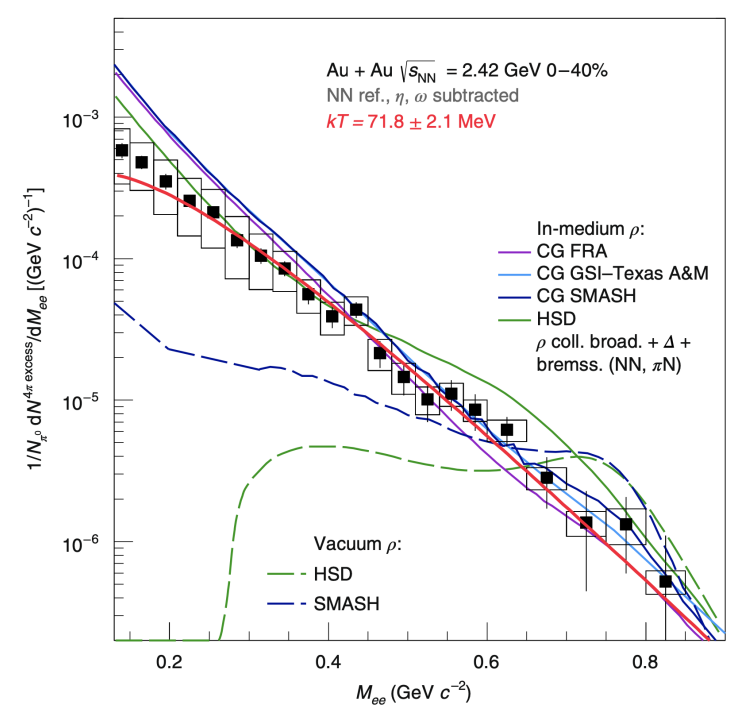

Figure 3.23: Acceptance-corrected dilepton excess yield from HADES for an invariant mass window of $0.15-0.85 \mathrm{GeV}$ confronted with different model calculations. Excess yield extracted by subtracting $\eta, \omega$ contributions as well as the $N N$ reference normalized to the number of neutral pions. Fig. 3 from [280].

Recently, the HADES collaboration confronted the presented predictions for the $\mathrm{AuAu}$ system with experimental data for an invariant mass window of $0.15-0.85 \mathrm{GeV}$ [280], which is most sensitive to the $\rho$ dilepton emission. While the available data is only able to probe the $\rho$ contribution, the same conclusions are reached as for $\mathrm{ArKCl}$. The agreement with experimental data, which appears to have lost any (resonance peak) structure for the large $\mathrm{AuAu}$ system, is greatly improved if the coarse-graining approach is employed. The $\rho$ contribution without medium modifications (blue dashed line, same as the blue solid line in Figure 3.18) alone overshoot the data for the total yield around the pole. The same is found for the HSD [162] approach without a $\rho$ in-medium broadening. Note, however, that the two "vacuum $\rho$ " contributions differ in the low-mass tail due to the in SMASH included contributions below the hadronic threshold. The trend of the experimental data of a rising low mass tail is therefore only observed for SMASH (see discussion in next section). An in-medium description, i.e. an explicit broadening of the spectral function, improves the description of the experimental data for all models with only small deviations remaining. Reassuring is the agreement between the here presented (CGSMASH) and the two other coarse-graining approaches [117, 253], which all employ the same underlying 
in-medium spectral function.

With the presented, albeit limited experimental data comparison, it is clear that the dilepton emission with vacuum resonance properties, which was shown in Figure 3.18, reveals an even larger overestimation in the intermediate-mass region in comparison to ArKCl. The in-medium modifications are more important in a larger medium due to the already observed overestimation in the $\rho$ pole mass region without summing overall contributions. The extension of the available experimental data to higher invariant masses will still be valuable to e.g. further constrain the $\phi$ production (see Section 3.2). Comparing to more differential predictions as presented below for $\mathrm{AuAu}$, will allow probing the dilepton and its medium effects in much greater detail.

\section{Comparison of the vector meson yields}

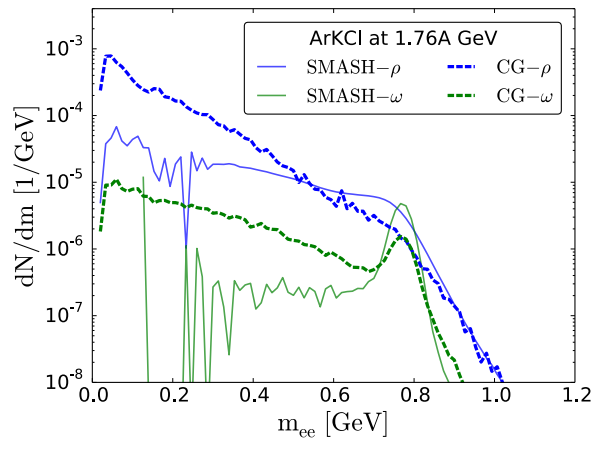

(a) $\mathrm{ArKCl}$

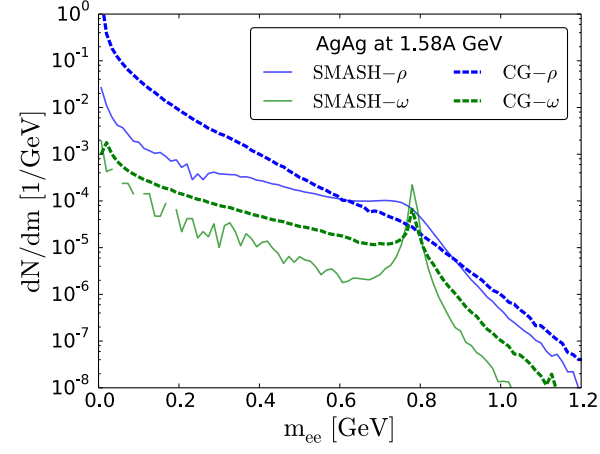

(b) $\mathrm{AgAg}$

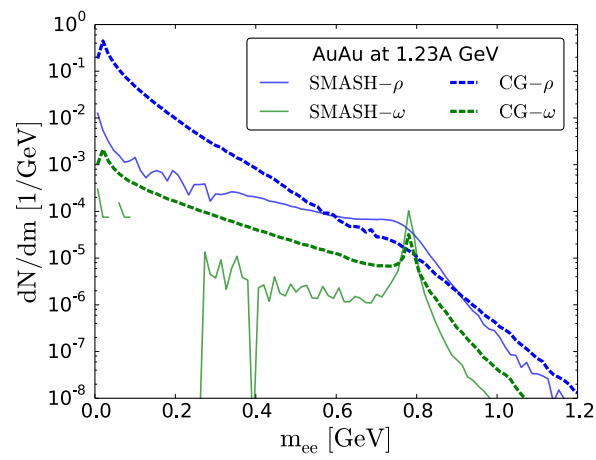

(c) $\mathrm{AuAu}$

Figure 3.24: Comparison of invariant mass spectra of dielectrons produced by $\rho$ and $\omega$ within the Coarse-Graining approach versus the default SMASH dilepton production.

The study presented here allows for a unique direct comparison between a coarse-graining approach and the dilepton production from a hadronic transport approach. This becomes possible since both rely on the same hadronic evolution. SMASH also includes low-mass vector meson contributions, which enable a comparison in this mass region. The comparison for the total yield was already shown above. This section focuses specifically on the vector meson ( $\rho$ and $\omega$ ) dilepton contributions, which are of most interest since an in-medium spectral function is employed for them. Therefore, this allows to contrast the different medium effects at play: On the one side, the transport approach with collisional broadening and vacuum resonance properties is shown, which includes the coupling of baryonic resonances to the vector mesons via Dalitz decays (cf. Figure 3.10). On the 
other side is a full in-medium description of the vector meson spectral functions.

Figure 3.24a shows the direct comparison between the vector meson yields for the smallest system of ArKCl. As expected from a broadening scenario of the vector meson spectral function, an enhancement of the low-mass tail together with a decrease in the pole mass region, especially for the $\rho$ yield, is observed. The $\rho$ yield from coarse-graining shows an almost exponential decrease with mass and dominates the spectrum over most of the covered invariant mass range. The $\omega$ yield is only around its pole mass equally large. Also, the broadening of the $\omega$ spectral function is nicely reflected in the dilepton emission around the peak.

Figure 3.24b and Figure 3.24c similarly show a shift from the pole masses in the $\rho$ and $\omega$ yield, in this case of the $\mathrm{AgAg}$ and $\mathrm{AuAu}$ system. Again, the broadening of the $\omega$ peak and the enhancement of the $\rho$ low-mass tail is nicely observed. The shape of the $\rho$ contribution almost completely flattens out and reveals an exponential decrease, whereas for the $\omega$ a dominating peak around the pole mass remains. As expected from the similar total dilepton spectra for both systems, the modification introduced from the coarsegraining approach is almost identical. All comparisons in Figure 3.24 interestingly reveal
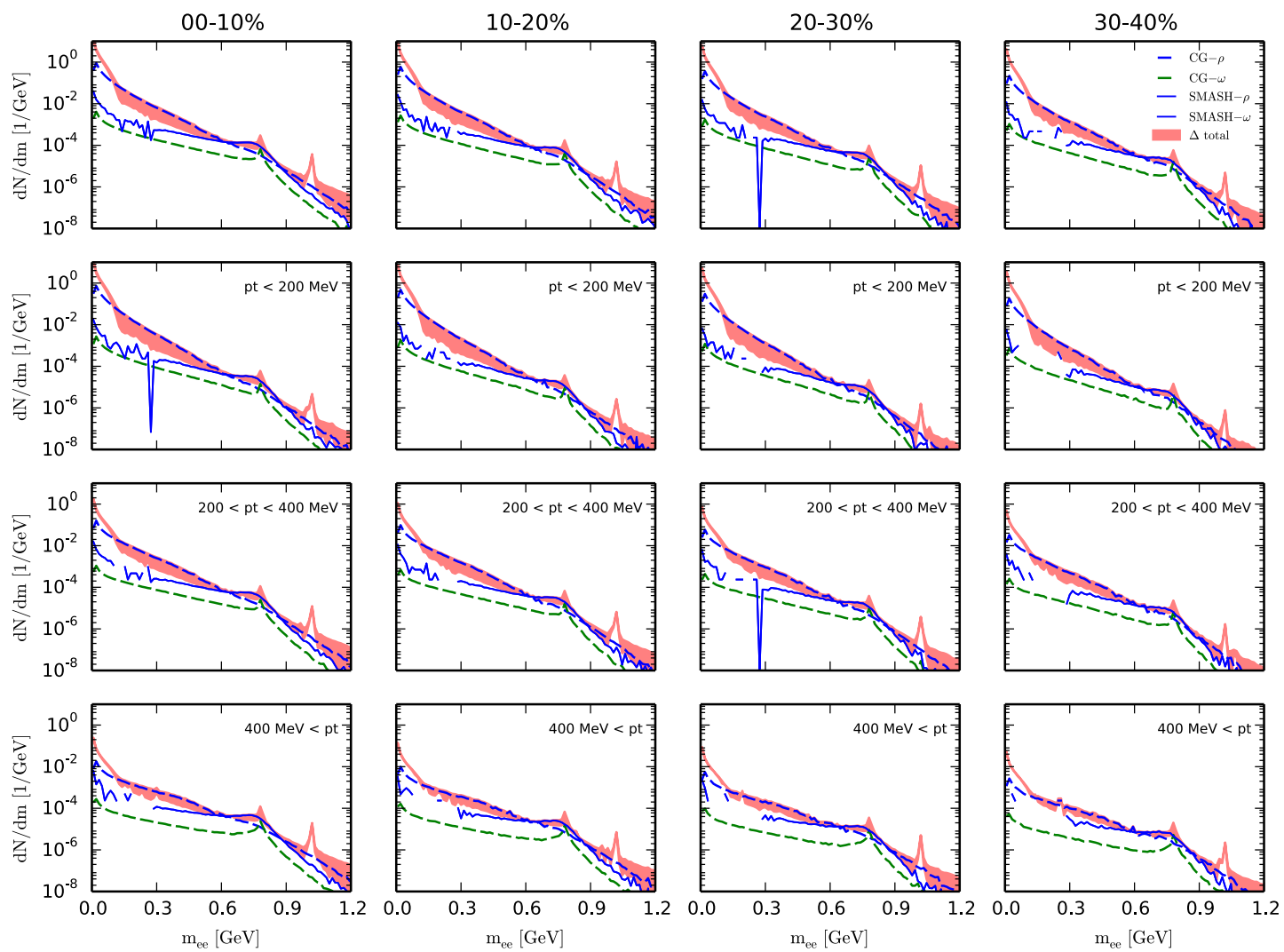

Figure 3.25: Invariant mass spectra of dielectrons produced by AuAu collisions at $E_{\mathrm{Kin}}=$ $1.23 A \mathrm{GeV}$ for different centrality classes (columns) and $p_{T}$ windows (no $p_{T}$ cut for top row). Only $\rho$ and $\omega$ contribution shown for Coarse-Graining approach (dashed lines) and the default SMASH dilepton production (solid lines). Difference in total emission marked as red area.

that the SMASH contributions obtain a low-mass tail similar to the CG contributions, which also appears in the experimental data above the $\pi$ dominated invariant region (cf. 

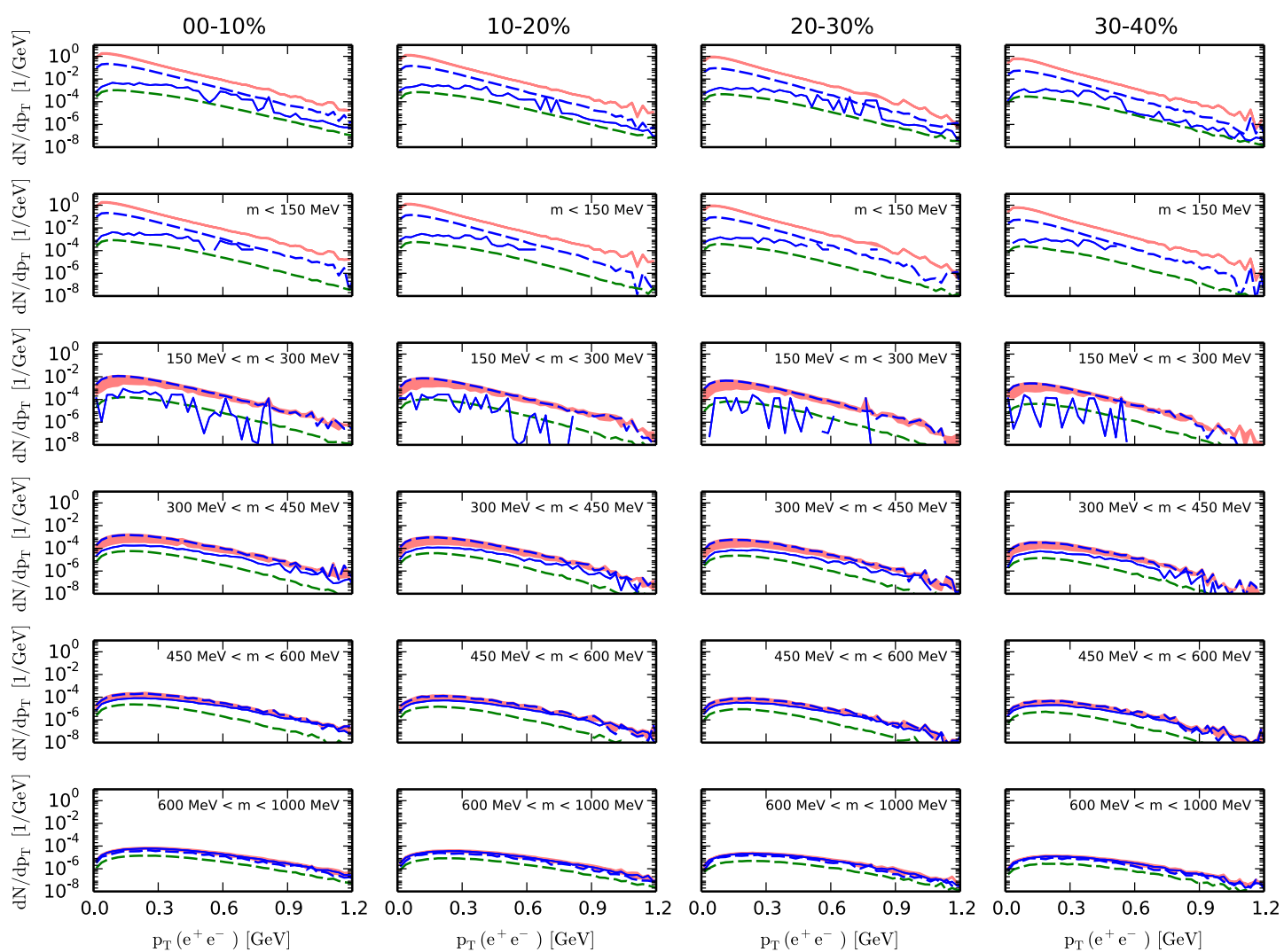

Figure 3.26: Transverse momentum spectra of dielectrons produced by AuAu collisions at $E_{\mathrm{Kin}}=1.23 \mathrm{~A} \mathrm{GeV}$ for different centrality classes (columns) and invariant mass windows (no invariant mass cut for top row). Same comparison between Coarse-Graining approach (dashed lines) and the default SMASH dilepton production as in Figure 3.25. Difference in total emission marked as red area.

Figure 3.20 or Figure 3.23). In SMASH, the Dalitz-like tail for low masses stems from the different baryonic-resonance contributions (compare Figure 3.10). Although this is not a medium effect, since it is already observed in proton-proton reactions, the underlying mechanism leading to the pronounced low-mass tail, namely the coupling of the vector meson to baryonic resonances, is the same that is found to be important for the in-medium modifications of the spectral functions used in the coarse-graining framework $[145,255]$. It is clear from the differences that collisional broadening plus baryonic Dalitz decays cannot account for the full effect of the medium. Nevertheless, the presented results quantify the different effects on the dilepton spectrum for the first time.

The isolate the difference further specific invariant masses, transverse momentum windows and centrality classes of the electron pairs are displayed in Figures 3.25, 3.26 and 3.27. The comparison shown in the figures displays the same contributions as Figure 3.24 of the $\rho$ and $\omega$ for the coarse-graining and the (vacuum) transport contributions ${ }^{3}$. In addition, the difference of the total emission between both approaches, caused by the already observed sensitivity to the medium effects, is marked in red. This allows to easily observe and further quantify the effect of the medium modifications of the vector mesons on the total

\footnotetext{
${ }^{3}$ The full results of the transport calculations for the $m_{e e}, p_{T}$ and $y$ spectra in the different centralities classes are provided in Appendix B for completeness.
} 

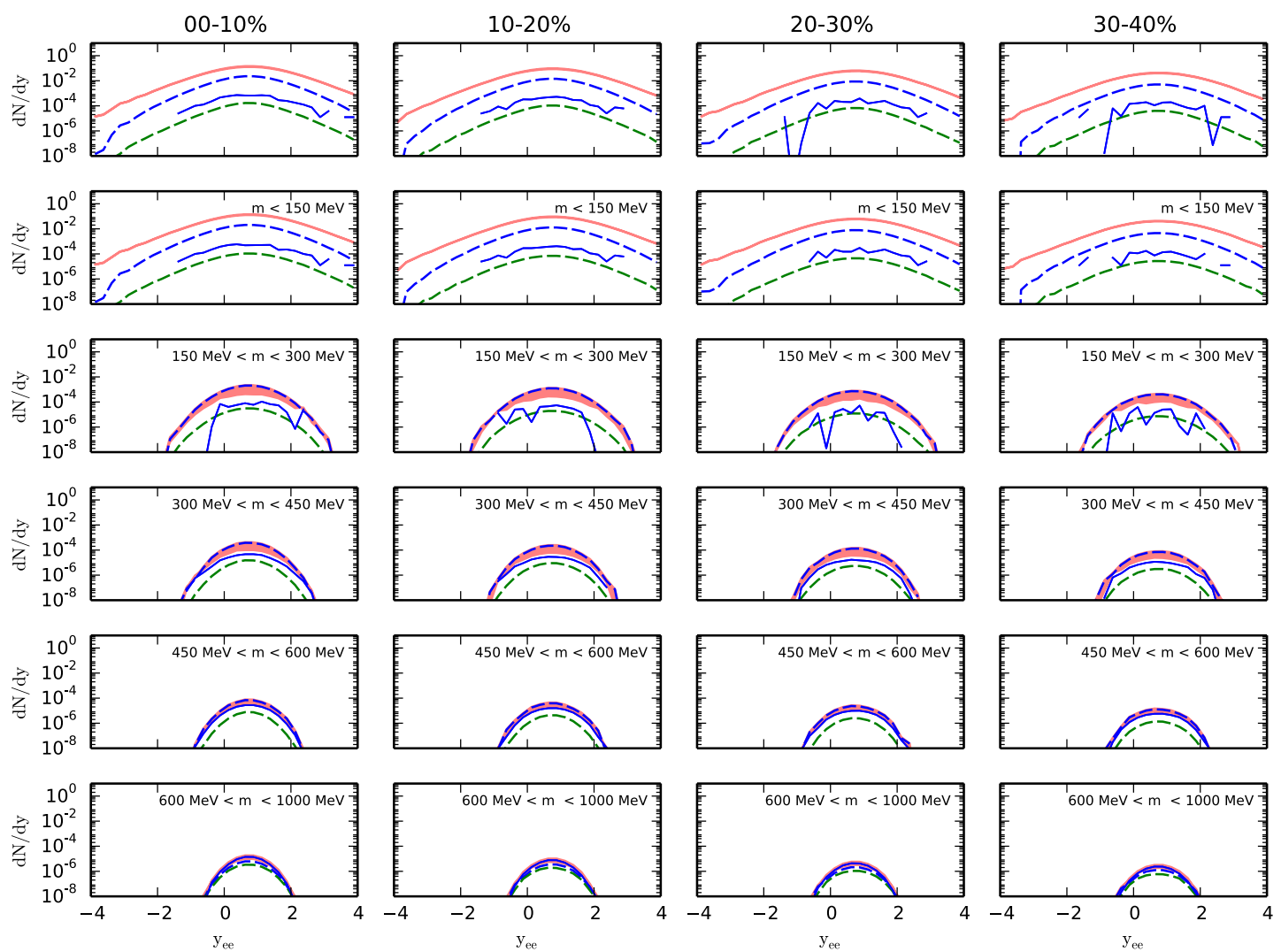

Figure 3.27: Rapidity spectra of dielectrons produced by $\mathrm{AuAu}$ collisions at $E_{\mathrm{Kin}}=$ $1.23 \mathrm{~A} \mathrm{GeV}$ for different centrality classes (columns) and invariant mass windows (no invariant mass cut for top row). Same comparison between Coarse-Graining approach (dashed lines) and the default SMASH dilepton production as in Figure 3.25. Difference in total emission marked as red area.

spectrum. The dependence on the centrality for the $m_{e e}, p_{T}$ and $y$ spectra is overall weak. More peripheral systems appear to be less sensitive. In Figure 3.25, the invariant mass spectra for lower momenta exhibit a slightly larger modification, which is a similar finding as shown for $\mathrm{pNb}$ in Figure 3.14. The effect of the medium in the presented calculations is most effectively isolated by splitting the spectra into different invariant mass windows as displayed in Figure 3.26 and Figure 3.27. A modification of the total yield is most pronounced for the 150 to $300 \mathrm{MeV}$ and 300 to $450 \mathrm{MeV}$ invariant mass region. Highquality experimental data in these phase-space regions would provide further constraints on the discussed modifications of the vector meson spectral functions.

\subsubsection{Summary}

The results in this section represent a comprehensive review of the complete set of available dielectron production measurements at SIS energies based on the hadronic transport approach SMASH. Dilepton spectra are shown to be sensitive to medium modification of the vector meson spectral function in larger systems already at the relatively low energies studied here. These results hint at an onset of chiral symmetry restoration, to which a changing vector meson spectral function is connected. 
The approach in general and the dilepton production in particular is validated by a good agreement with experimental data in proton-nucleus and nucleus-nucleus collisions up to a system size of CC reactions. Binary interactions and vacuum resonance properties are sufficient to describe the hadronic dynamic for these small systems. The agreement originates from the solid description of elementary pp collisions. Only for a low kinetic energy around $1 \mathrm{GeV}$ is an underestimation for quasi-free np collisions and subsequently for $\mathrm{CC}$ observed. The dilepton decays are taken into account for the spectral function calculation for all vector mesons $(\rho, \omega, \phi)$. The subsequent contributions, down to $2 m_{e}$ below the hadronic threshold, are significant for the low-mass region and become more prominent the larger the collision system is.

The hadronic transport approach is complemented by the coarse-graining approach based on the same hadronic evolution to study the sensitivity of the invariant mass spectrum to in-medium modifications of the vector meson spectral function for the larger systems ( $\mathrm{ArKCl}, \mathrm{AgAg}$ and $\mathrm{AuAu}$ ). Both approaches reveal similar features in their dilepton contributions, including the low-mass tail. Nevertheless, the transport description, including the coupling to baryons and collisional broadening, cannot account for the necessary significant modifications visible in larger collision systems caused by an in-medium description of the vector meson spectral function. An agreement with the available experimental data is only observed if an in-medium description is employed, indicating the dilepton spectrum's sensitivity to a change in the vector meson spectral function.

The well-understood dielectron production is furthermore employed to predict upcoming experimental results. $\pi \mathrm{p}$ is demonstrated to specifically probe the coupling of the $\rho$ meson to the $N^{*}(1520)$ baryonic resonance. AgAg is found to be overall very similar to the $\mathrm{AuAu}$ system suggesting that the dilepton production behaves the same for smaller systems with higher energies compared to larger systems with lower beam energy. The main source for the dilepton emission for invariant masses higher than the $\phi$ pole mass in AgAg, is the $\rho$ contribution originating from (two) $\pi$ annihilations. Similarly, the multi- $\pi$ contribution is dominant beyond the $\phi$ peak in the coarse-graining approach.

Overall, the ability of the hadronic transport approach to successfully describe the dilepton production in small systems and to constrain additional medium effects in larger systems is demonstrated. 


\subsection{Strangeness production mechanism}

This section is based on [2] and [3].

This section probes the production mechanisms of strange hadrons as another complementary hadronic observable of special interest (see also Section 1.3.2) after studying the dilepton emission above, which offers an unperturbed view into the in-medium resonance properties. Again the hadronic transport approach SMASH is employed to provide a cascade calculation with vacuum properties. Similar to the dilepton results, using a cascade calculation without medium effects like potentials allows constraining the excess for additional in-medium modifications in the experimental data. The low beam energy kinematic regime reached by the discussed SIS-18 experiment HADES is close to the production threshold [173], making it particularly sensitive to production mechanisms since secondary reactions become necessary for the particle production. Experimentally, strangeness production at low energies is enhanced in nucleus-nucleus reactions compared to elementary reactions $[68,174]$. Upcoming results for the AgAg collision system will allow to further understand the production of strange hadrons by offering experimental data at an intermediate size but with slightly higher energy than the previously taken AuAu data at $E_{\mathrm{Kin}}=1.23 \mathrm{~A} \mathrm{GeV}$. Therefore, strangeness production in $\mathrm{AgAg}$ is a focus in this section.

In the following, the strangeness production with the transport approach SMASH is first introduced in general by showing the comparison of $K$ mesons, as the most abundant strange particle, to experimental data and discussing the scaling of strange hadrons with the number of participants. Then in the second part of this section, the theoretical discrepancies to data for the few $\mathrm{GeV}$ energy regime for the $\Xi$ baryon and the $\phi$ meson are discussed. Much higher yields were observed than expected by thermal model calculations [68] (see also Figure 1.11). There are several attempts to explain these high production yields $[175,176,177]$. This section focuses on one possible explanation for the high production yield of the $\Xi$ baryons and $\phi$ mesons. Their discrepancies compared to thermal model calculations might be explained by their production in decays of high mass nucleon resonances. This mechanism is based on the idea in [177] and will be first constrained in elementary reactions after being applied to larger systems. In this sense, the $\Xi$ and $\phi$ yield is predicted without explicit medium effects and solely based on the additional production mechanism.

For the $\Xi$ baryon, predictions are provided for the AgAg system based on a constraint of the decay probabilities from experimental data for elementary reactions. They will allow constraining the viability of the $\Xi$ (and $\phi$ ) production from high mass resonance decays. The found branching ratio is verified by comparisons to existing experimental data for ArKCl collisions.

\subsubsection{Kaon production and $N_{\text {part-scaling }}$}

The $K$ production is discussed first in this section ${ }^{4}$. It is directly related to the $\Xi$ and $\phi$ production, since the dominant decay of the $\phi$ meson $(\phi \rightarrow K \bar{K})$ is an important production (so called feed-down) mechanism for the antikaons. Constraining the $\phi$ production itself, thereby simultaneously constraints the (anti-) kaon production. The other two processes that produce (anti-) kaons in SMASH are (i) decays of baryonic resonances $\left(N^{*}, \Delta^{*}\right)$ into hyperon $(Y)$ and kaon $\left(N^{*}, \Delta^{*} \rightarrow Y K\right)$ and (ii) strangeness exchange in a

\footnotetext{
${ }^{4}$ The results in this section are given as context for the strangeness production in general and are taken from [2] and [3]. Original contributions of this thesis are found in the following Section 3.2.2 only.
} 

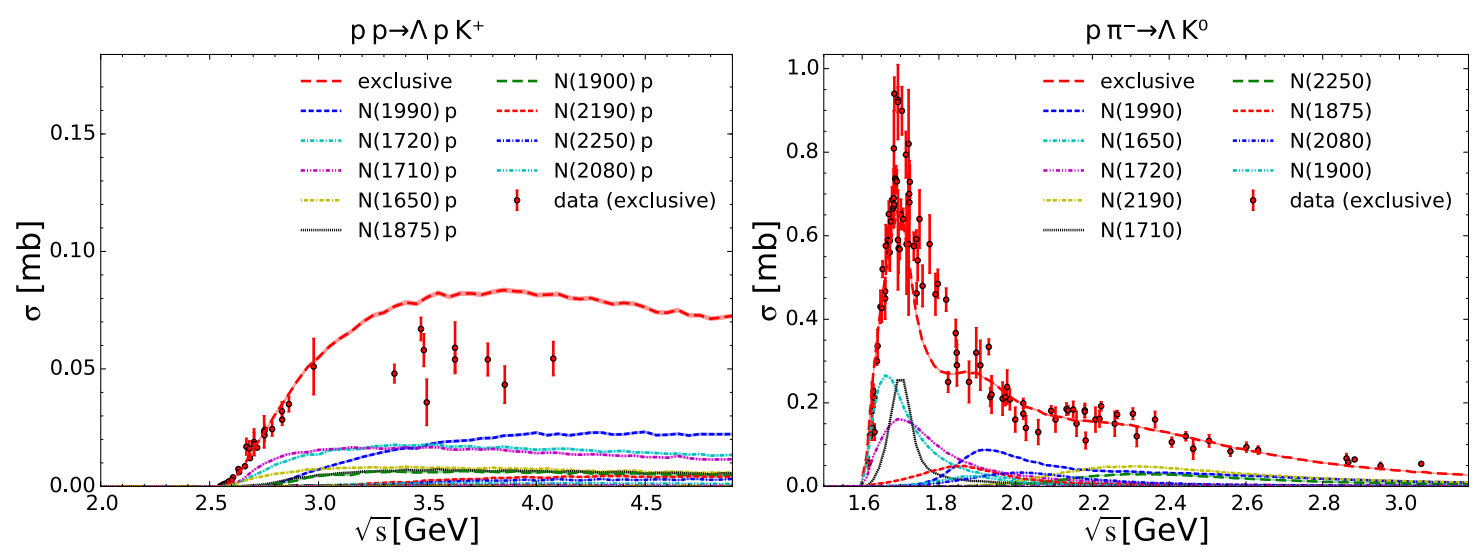

Figure 3.28: Exclusive production cross-section for $p p \rightarrow \Lambda p K^{+}$and $p \pi^{-} \rightarrow \Lambda K^{0}$. Experimental data from $[281,282,283,284,285,286,287,288,289,290]$. Figure taken from [2].
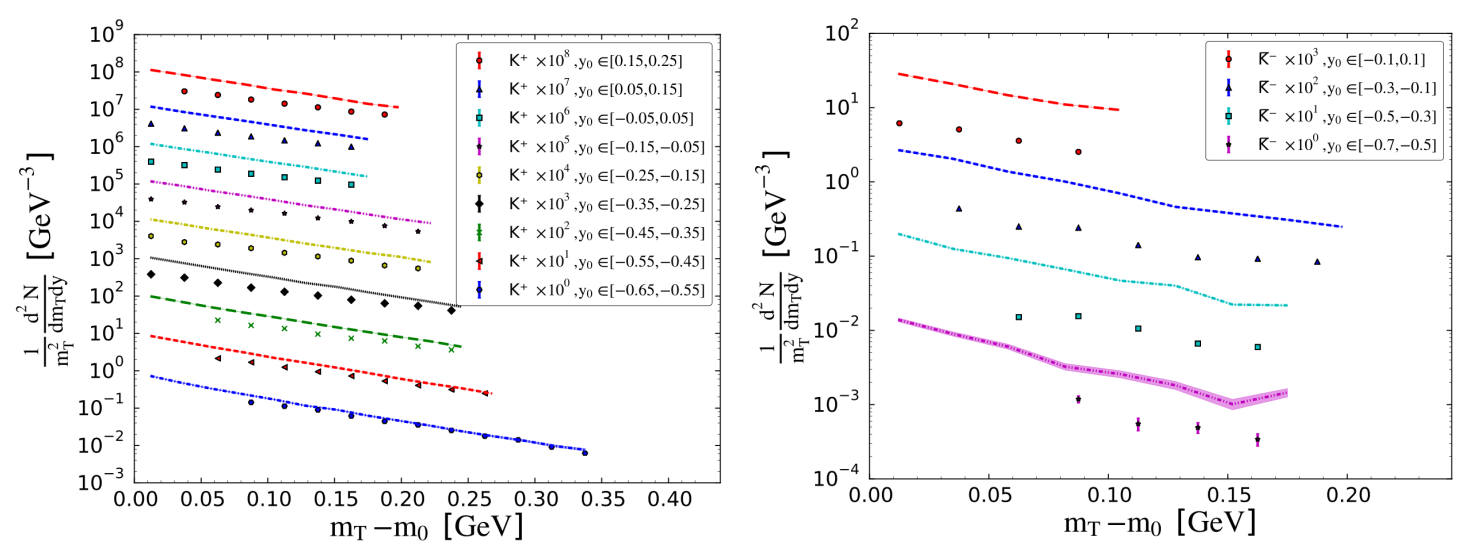

Figure 3.29: $m_{T}$ spectra of $K^{+}$and $\bar{K}^{-}$in AuAu collisions at $E_{\text {Kin }}=1.23 \mathrm{GeV}$. Experimental data from [292].Figure taken from [2].

pion-hyperon scattering $(\pi K \rightarrow \bar{K} N)$, which mostly is realized via a hyperon resonance state $Y^{*}\left(\pi K \rightarrow Y^{*} \rightarrow \bar{K} N\right)$ (see Section 2.2.3).

The $K$ production in SMASH is carefully constrained by comparisons with elementary cross-section [2]. An example of two important cross-sections for $K$ production is shown in Figure 3.28. The cross-section is overall comparable with the available experimental data. Some energy regions are not perfectly described. Branching ratios of $N^{*}, \Delta^{*}, Y^{*}$ that are involved in the $K$ production are varied within the PDG limits until a satisfactory agreement is achieved. In future work, such a process could also be automated and improved by a Bayesian fit [291]. The agreement of $K$ production in heavy-ion collision in the few $\mathrm{GeV}$ energy range is not as good as illustrated by Figure 3.29, which shows a data comparison for AuAu collisions at $E_{\text {Kin }}=1.23 \mathrm{GeV}$. While the $K^{+}$at large rapidities agrees with the data, at mid-rapidity, an overproduction is found. The same is true for $\bar{K}^{-}$in all rapidity windows. The observed overproduction either reflects the remaining discrepancies in the elementary cross-section or hints that additional medium effects have a significant influence. Possible in-medium effects include modifications to cross-sections or nucleon-kaon potentials, which are known to affect the strangeness production [164]. Furthermore, a hyperon-nucleon potential [293] based on the qualitative features of a chiral 
effective theory at the next leading order could potentially be employed. For the presented results, only Fermi motion is employed. Nucleon-nucleon potentials are neglected since they are found not to influence the strangeness production.

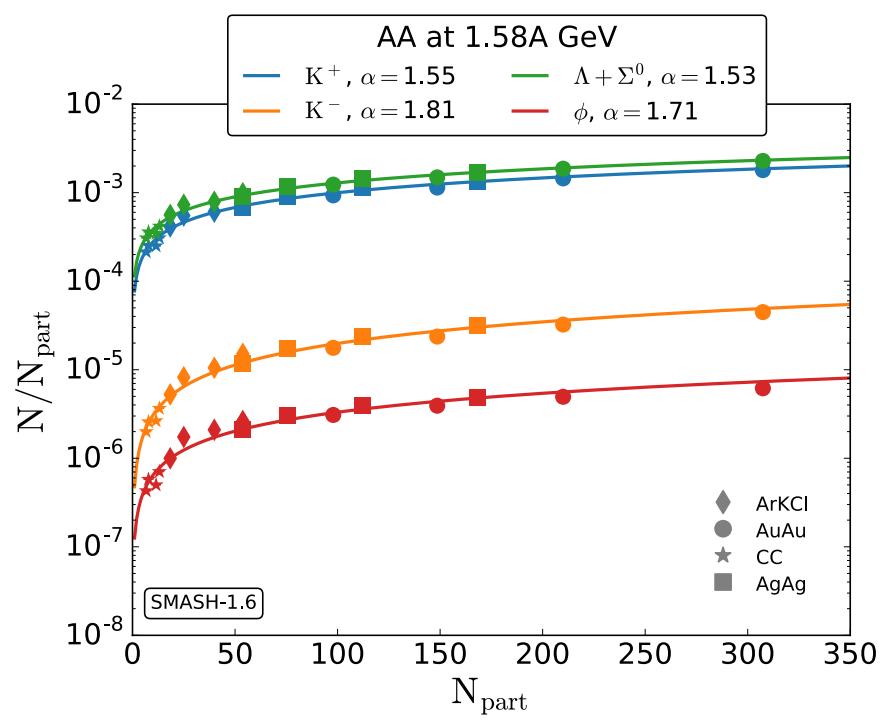

Figure 3.30: Multiplicities per number of participants $\mathrm{N} / \mathrm{N}_{\text {part }}$ as a function of $\mathrm{N}_{\text {part }}$. Four centrality classes $(0-10 \%, 10-20 \%, 20-30 \%, 30-40 \%)$ are used for each system. Solid lines are fits for the function $\mathrm{N} \propto\left(\mathrm{N}_{\text {part }}\right)^{\alpha}$. Figure taken from [3].

To put the strangeness production for SMASH (version 1.6) into an even larger context, Figure 3.30 shows the particle yield $N$ (over $N_{\text {part }}$ ) for a selection of strange particles, including $K$, for a large set of systems and centrality classes. The centrality is characterized by the number of participants $N_{\text {part }}$. Impact parameters and $N_{\text {part }}$ for each centrality class are determined using Glauber calculations provided by [294].

The results are fitted using the method of least squares to the function

$$
\frac{N}{N_{\text {part }}}=C \cdot\left(N_{\text {part }}\right)^{\alpha-1}
$$

with $\alpha$ being the fitting parameter of interest here.

This scaling behavior for $N_{\text {part }}$ is chosen to compare $\alpha$ with results from [173] for AuAu and allows to study if a universal system size dependence can be observed. The universal value reported in [173] is $\alpha^{\exp }=1.45 \pm 0.06$. In [173] three other transport approaches were compared to the experimental data. The values for SMASH are added here including 3 more collisions systems (see Figure 3.30). They deviate from the data by a maximum of $20 \%$ with $\alpha_{\Lambda+\Sigma^{0}}$ being the closest to the experimental value. The deviations of $\alpha$ for the different particle species could be seen as a systematic uncertainty. Note that the calculations here do not take any potentials into account and represent a cascade baseline. Since the $\alpha$ parameter at least for $K$ mesons is sensitive to the stiffness of the EoS, when potentials are employed, as found in [164], deviations between the experimental value and the transport approach could change with the inclusion of potentials. Furthermore, using the model-dependent $N_{\text {part }}$ value by the Glauber model could also explain differences for $\alpha$.

Overall, Figure 3.30 illustrates that the strange particle multiplicities exhibit the previ-

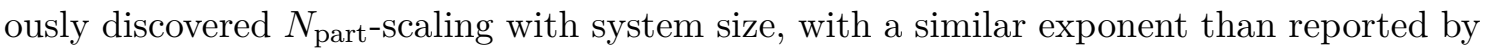



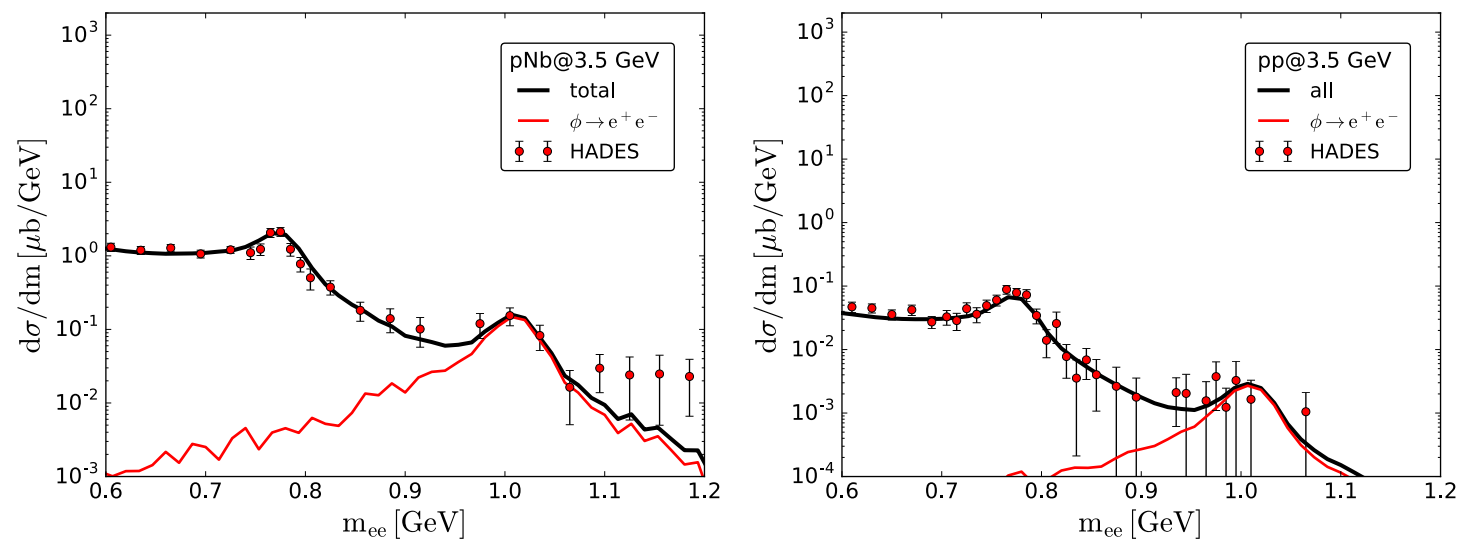

Figure 3.31: Invariant mass spectrum of dielectrons produced by $\mathrm{pNb}$ and pp reactions at $E_{\text {Kin }}=3.5 \mathrm{GeV}$. Total and $\phi \rightarrow e e$ contribution with added $N^{*} \rightarrow \phi N$ decays. Experimental data from [156].

the HADES collaboration [173] .

\subsubsection{Production of $\phi$ and $\Xi$ from the decay of heavy $N^{*}$ resonances}

The additional production mechanism studied here in detail, follows the idea from [177] and extends the SMASH approach by adding new decay channels for heavy $N^{*}$ resonances that produce $\phi$ and $\Xi$. The two added decay channels are $N^{*} \rightarrow N \phi$ and $N^{*} \rightarrow \Xi K K$. They enhance the production of both species. This is necessary since the yield is underpredicted for both in comparison to experimental data. One of the main aims of this work is to predict the $\phi$ and $\Xi$ yields with this mechanism after constraining them with available elementary data. The comparison with upcoming experimental data will show if these decays are a potential source of the seen excess, particularly for the $\Xi$. Note that the same general idea of constraining with experimental data of elementary systems is followed for the $K$ production and as reported above, partly unsuccessful. However, for the here proposed mechanism first results for larger systems ( $\mathrm{ArKCl}$ ) already look promising, as described below.

\section{$\phi$ production}

Since the experimental data for the $\phi$ production in elementary systems is only available close to the production threshold, results for the dilepton production are employed as the main constraint for elementary reactions here, which are in detail described in the previous section. The invariant mass spectrum allows to identify a peak around the $\phi$ pole mass, produced by the $\phi \rightarrow e^{+} e^{-}$decay that is directly related to the number of produced $\phi$ in the collision.

Figure 3.31 shows the strong $\phi$ peak that surfaces around $1 \mathrm{GeV}$ in the invariant dielectron mass spectrum for the pNb system at $E_{\text {Kin }}=3.5 \mathrm{GeV}$ (left plot). From the depicted total yield, it is clear that the $\phi$ contribution is dominant in this region (see also the result with all contributions in Figure 3.13). Therefore the good-quality experimental data points for this invariant mass are used to constrain the $N^{*} \rightarrow \phi N$ branching ratios for all nucleon resonances with a pole mass of $2080 \mathrm{MeV}$ or higher. The found value for the fixed branching ratio for SMASH-1.1 is 0.005, which is larger than the value reported in [295]. The branching leads to an agreement with experimental data, as Figure 3.31 


\begin{tabular}{ccc}
\hline $\mathrm{N}\left(\Xi^{-}\right)$ & $\mathrm{pNb}$ & $\mathrm{ArKCl}$ \\
\hline SMASH-1.6 & $\approx 0.0$ & $6.25 \times 10^{-7}$ \\
$\Xi^{-}$from N* & $2.04 \times 10^{-4}$ & $1.95 \times 10^{-4}$ \\
HADES & $(2.0 \pm 0.4 \pm 0.3) \times 10^{-4}$ & $(2.3 \pm 0.9) \times 10^{-4}$ \\
\hline
\end{tabular}

Table 3.1: $\Xi^{-}$yield in pNb at $E_{\text {Kin }}=3.5 \mathrm{GeV}$ and $\mathrm{ArKCl}$ at $E_{\mathrm{Kin}}=1.76 \mathrm{~A} \mathrm{GeV}$ collisions. HADES experimental data from [296, 174].

shows. The pA system is the most elementary system available as constraint, which means medium effects like absorption also play a role. Unfortunately, due to large error bars, the corresponding elementary pp data that would offer a clean vacuum reference only provides an upper bound in the $\phi$ peak mass region. Nevertheless, the obtained value is consistent with this bound since an agreement within error bars is seen in the right plot of Figure 3.31. The same is true for the larger $\mathrm{ArKCl}$, where the data is also not sufficient for a constraint (cf. Figure 3.16).

Predictions for the dilepton prediction with this branching ratio for the $\phi$ peak for larger systems were already presented for $\mathrm{AgAg}$ and $\mathrm{AuAu}$ in Figure 3.17 and 3.18. For the higher measured beam energy in AgAg collisions, the peak is more pronounced in the spectrum. Upcoming experimental data at the peak will therefore be valuable to further constrain the $\phi$ production and the $N^{*} \rightarrow N \phi$ decay as its production mechanism.

\section{$\Xi$ production}

Theoretical approaches famously underpredict production of $\Xi$ baryons [174]. SMASH without an extension for the $\Xi$ production is no exception as can be seen in Table 3.1 and 3.2 (results for SMASH-1.6 [211]). In SMASH (version 1.6), the $\Xi$ is produced by the decay of heavy hyperon resonances like the $\Sigma(2030), \Lambda(2100)$ or resonances of the $\Xi$ itself. They are formed either by nucleon-kaon or hyperon-meson scatterings (see Section 2.2.3) making the production of $\Xi$ rare since these are secondary scatterings with partners that are not abundant during a collision. In addition, the decaying resonances are heavy and the branching ratios for decays involving the $\Xi$ are small, which explains the underprediction for the $\Xi$ multiplicity.

Therefore, the approach is extended by adding new decay channels for heavy $N^{*}$ resonances, namely $N^{*} \rightarrow \Xi K K$ as described above. The resonance treatment in SMASH (see Section 2.1.1) prevents adding any decays for which the combined pole mass of the final state particles is larger than the pole mass from the decaying resonance, although such a decay would be strictly physically speaking possible. This restricts the addition of the new decay channel to the heaviest $N^{*}$ resonances. The decay channel is added to the two heaviest resonances $(N(2220), N(2250))$ and their pole mass is shifted slightly upwards $(N(2220) \rightarrow N(2350), N(2250) \rightarrow N(2400))$, which is possible due to their relatively large width $(\Gamma \geq 400 \mathrm{MeV})$ and experimental uncertainties for the pole masses (on the order of $100 \mathrm{MeV})$.

The branching ratio for the new $N^{*} \rightarrow \Xi K K$ decay is now constrained with the experimental data from $\mathrm{pNb}$ reactions, which is the most elementary data available. The result is $B R\left(N^{*} \rightarrow \Xi K K\right)=0.5$. The results for $\mathrm{pNb}$ before and after the addition of the $\Xi$ production from heavy $N^{*}$ decays are shown in Table 3.1. Before, although theoretically possible, no $\Xi$ production (for the given number of calculated reactions) is observed due to 


\begin{tabular}{ccc}
\hline$\Xi^{-} /\left(\Lambda+\Sigma^{0}\right)$ & $\mathrm{pNb}$ & $\mathrm{ArKCl}$ \\
\hline SMASH-1.6 & $\approx 0.0$ & $1.1 \times 10^{-5}$ \\
$\Xi^{-}$from N* & $1.5 \times 10^{-2}$ & $4.5 \times 10^{-3}$ \\
HADES & $(1.2 \pm 0.3 \pm 0.4) \times 10^{-2}$ & $(5.6 \pm 1.2 \pm 1.8) \times 10^{-3}$ \\
\hline
\end{tabular}

Table 3.2: $\Xi^{-} /\left(\Lambda+\Sigma^{0}\right)$ ratio in $\mathrm{pNb}$ at $E_{\mathrm{Kin}}=3.5 \mathrm{GeV}$ and in $\mathrm{ArKCl}$ at $E_{\mathrm{Kin}}=1.76 \mathrm{~A}$ $\mathrm{GeV}$. HADES experimental data from $[296,174]$.

the lack of many secondary reactions in the small pNb system. After the addition of the new channel with the tuned $B R\left(N^{*} \rightarrow \Xi K K\right)$ of 0.5 , the experimental data from HADES [296] is matched. The same is true for the $\Xi^{-} /\left(\Lambda+\Sigma^{0}\right)$ ratio as seen in Table 3.2. Compared to [177] a larger branching ratio is reported here since the decay is included for less and heavier resonances. A similar observation was made for the branchings of $N^{*}$ decays into $\phi$ above. Interestingly, if one calculates the ratio of the two branching ratios of $\phi$ and $\Xi, B R\left(N^{*} \rightarrow \phi N\right) /\left(B R\left(N^{*} \rightarrow \Xi K K\right)=0.02\right.$, the relation exactly matches for the two branching ratios reported in [177]. Note that, to calculate the ratio, the $B R\left(N^{*} \rightarrow \phi N\right)$ for SMASH-1.6 [211] needs to be slightly modified to 0.01 in comparison to the quoted results above for SMASH-1.1. This becomes necessary since other branchings are updated in SMASH-1.6 [211] with values from the PDG [256].

Next, the $\Xi$ production is compared to available experimental data for a larger system to verify the newly introduced treatment. Tables 3.1 and 3.2 show that the $\Xi$ production in $\mathrm{ArKCl}$ is also matched well within the errors, which suggests that in-medium effects might not be as significant on the $\Xi$ as on the $K$ production mechanisms.

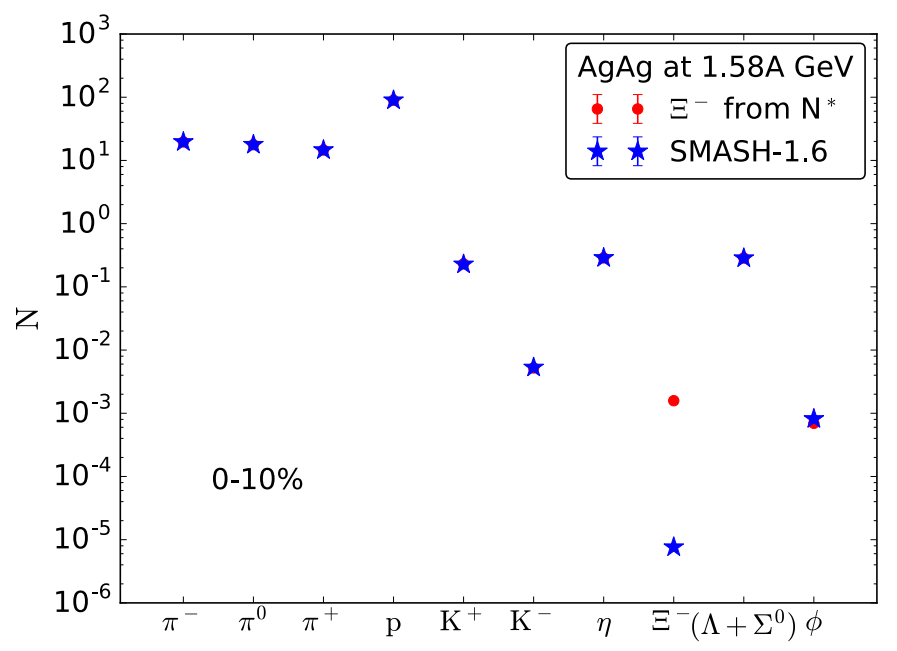

Figure 3.32: Results for the particle production yields for $\mathrm{AgAg}$ collisions at $E_{\text {Kin }}=1.58 \mathrm{~A}$ $\mathrm{GeV}$ at $0-10 \%$ centrality from SMASH-1.6 [211] (stars) compared to the modified branching ratios for high mass resonances (circles). Figure from [3].

On this basis, the $\Xi$ production for AgAg collisions is calculated, which matches the upcoming experimental data set from HADES. Predictions for the most relevant particle yields in $\mathrm{AgAg}$ collisions at $E_{\mathrm{Kin}}=1.58 \mathrm{~A} \mathrm{GeV}$ in the $0-10 \%$ centrality class are depicted in Figure 3.32 to provide the context for the specific study of strange particles. The results from SMASH-1.6 [211] (stars) are confronted with the yields resulting from the version, including additional $N^{*} \rightarrow \Xi K K$ resonance decays (circles). The most abundant 
particles in low-energy collisions are protons and pions with a charged pion ratio $\pi^{+} / \pi^{-}$ that is smaller than 1 reflecting the isospin imbalance of the $\mathrm{AgAg}$ collision system. All strange particle multiplicities are smaller since more energy is needed to produce their higher masses and newly formed strange quarks. Figure 3.32 also shows that the addition of $N^{*}$ resonances decaying into $\Xi^{-}$-baryons only influences the $\Xi^{-}$yield itself significantly. The $\phi$ meson yield is also slightly altered since adding a new decay channel lowers the weight of the existing channels. The effect is fortunately small since the $\Xi^{-}$is merely produced once in 1000 collisions while the production rates of the other particles are significantly higher. The predicted values for multiplicity and $\Xi^{-} /\left(\Lambda+\Sigma^{0}\right)$ ratios are also explicitly given for future reference and comparison to the upcoming experimental data in Table 3.3. Since the $\Xi^{-}$is produced in a multi-step process similar to $K^{+}$[164], the

\begin{tabular}{ccc}
\hline \hline $\operatorname{AgAg}$ & $\mathrm{N}\left(\Xi^{-}\right)$ & $\Xi^{-} /\left(\Lambda+\Sigma^{0}\right)$ \\
\hline SMASH-1.6 & $8.50 \times 10^{-6}$ & $2.71 \times 10^{-5}$ \\
$\Xi^{-}$from $\mathrm{N}^{*}$ & $1.78 \times 10^{-3}$ & $5.63 \times 10^{-3}$ \\
\hline \hline
\end{tabular}

Table 3.3: Predictions for $\Xi^{-}$yield and $\Xi^{-} /\left(\Lambda+\Sigma^{0}\right)$ ratio in $\mathrm{AgAg}$ collisions at $E_{\mathrm{Kin}}=$ $1.58 \mathrm{~A} \mathrm{GeV}$.

yield is sensitive to the employed treatment of the Fermi motion, the resonance lifetime and the underlying equation of state for the potentials, which influences the density near the collision center and in turn also the resonance lifetimes. For the results here, the same treatment established in other studies with SMASH (other results in this work and [98, 195]) without potentials is chosen to limit the uncertainties and make the calculation numerically less expensive. 


\section{Summary}

Overall, the approach to produce $\phi$ mesons and $\Xi$ baryons from heavy $N^{*}$ resonance decays is able to describe them, by other theoretical approaches underpredicted, data from HADES with only one free parameter. A branching ratio of 0.005 for the $N^{*} \rightarrow$ $\phi N$ decays (for SMASH-1.1 and 0.001 for SMASH 1.6) and 0.5 for the $N^{*} \rightarrow \Xi K K$ decays (SMASH-1.6 [211]) is found to lead to an agreement with the experiment. The $\phi$ dilepton contribution matches the data in pp, pNb and ArKCl. The $\Xi$ multiplicity and the $\Xi^{-} /\left(\Lambda+\Sigma^{0}\right)$ ratio agree for pNb as well as ArKCl. Interestingly, the relation between the $N^{*}$ decay branching ratios into $\phi$ and $\Xi$ is the same as found in previous studies with other approaches, even though absolutely they are both higher. Note that the available data is matched with the cascade calculation based on vacuum properties, leaving no excess that could be attributed to medium effects. For the discussed $K$ production, this

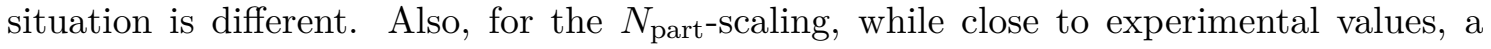
sensitivity to additional medium effects like potentials is not ruled out. The comparison of the predictions in this work with future data, particularly in $\mathrm{AgAg}$ collisions, will give further insights into the origin of the $\phi$ and $\Xi$, their production mechanism, and the role of potentials. 



\section{Chapter 4}

\section{Stochastic rates}

In this chapter, collision probabilities for different reactions are directly derived from the collision integral of the Boltzmann equation. The necessary theoretical considerations are presented in a comprehensive pedagogical fashion (Section 4.1). Results, in particular, include the collision probability for multi-particle reactions like 3-to-1, 3-to-2 and 5-to-2 reactions. In addition, the derivations are generalized for n-to-1, n-to- 2 and arbitrary n-to-m reactions, which can be used in the future to extend the approach to other reactions. The presented equations are the foundation of a stochastic interpretation of the microscopic scattering process in a particle gas (stochastic rates or local ensemble method), where they are used to determine whether and when particles collide. Such a stochastic collision criterion is introduced in a hadronic transport approach (Section 4.2), which is an idea already explored in the literature $[134,126,128,111,109,129,130]$. The application of the stochastic collision criterion for specific multi-particle reactions in the transport approach is discussed at the end of the chapter (Section 4.3). The employed multi-particle reactions are the mesonic Dalitz decay back-reaction, the deuteron catalysis and the nucleon-antinucleon annihilation back-reaction. They are of particular interest for the following studies (in Chapter 5) and enable to address multiple topical research questions (Section 5.3 and 5.4). Studying reactions with more than two incoming particles in the following chapter, after presenting the theoretical basis and description within a transport approach here, is the main topic of this thesis.

Transport approaches usually employ a distance criterion [106, 221, 222] based on a geometric interpretation of the cross-section (see Section 2.2.2). The stochastic rates approach provides an equivalent treatment for two-particle reactions with three distinct advantages. First, the equations for stochastic rates are inherently boost-invariant. Second, the collision probabilities are theoretically rigorously based on the fundamental (Boltzmann) equation of the underlying (kinetic) theory. Third, since no generalization exists for the distance between more than two particles scattering, the collision integral for geometric criteria is truncated to only include binary collisions. The collision probability, on the other hand, is straightforward to generalize to multi-particle reactions.

Before presenting the derivation, the context of the new stochastic rates approach in the literature is discussed. Compared to previous studies on stochastic rates, the framework introduced here provides an extension and mainly differs in the employed reactions. The idea of a stochastic collision criterion is introduced in [134] and [126]. In [134], the local ensemble method is introduced with a focus on the optimization of computing time and only applied to two-body reactions. The authors of [126] applied the method to multi- 
particle reactions for the first time. Similar as it is done in this work, they also studied deuteron production. The approach presented here and the one from [126] both include the deuteron catalysis reaction involving nucleons: $N p n \rightarrow N d$. For the low energies discussed in [126] this reaction is likely sufficient since $N$ dominates the system. But, for higher (intermediate) beam energies (as studied in Chapter 5) the catalysis reaction have to be extended with the pion catalysis reaction: $\pi p n \rightarrow \pi d$ [184], similar to a recent study for small collision systems at high beam energies [127]. Other authors focus on the $B \bar{B}$ annihilation reactions that produce multiple mesons $[128,129,130]$ with the PHSD (Parton-Hadron String Dynamics) approach [240]. In their quark rearrangement model for baryon-antibaryon annihilation and reproduction, all reactions follow the scheme $B \bar{B} \leftrightarrow 3$ mesons. In this way, a large set of different reactions can be included. The later discussed $N \bar{N} \leftrightarrow 5 \pi$ is therefore modeled as a chain of reactions in this approach. On the parton level, stochastic rates are studied with the parton transport approach BAMPS (Boltzmann Approach of Multi-Parton Scatterings), including the $g g g \leftrightarrow g g$ gluon bremsstrahlung reactions [111]. The hadronic transport approach GiBUU also allows employing a probabilistic collision criterion for three-body reactions (e.g. 3-to-1) [109].

The derivation compiled in the following Section 4.1 resembles and combines notation and ideas from the ones presented in [128, 111]. Compared to [111], the absence of a known scattering matrix element makes additional steps necessary before employing the derived probabilities. A similar idea as in [128] is followed, where the scattering matrix element is assumed to only depend on the initial center-of-mass energy. This approach differs from the idea in [126], where the matrix element is factorized into two terms as an approximation. This factorization results in a different formula for the 3-to-2 collision probability (cf. Section 4.1.10) containing the two-body $N N$ cross-section and a momentum dependent volume [126]. While also making an assumption about the matrix element, the approach presented here is more general because it allows treating all processes for which the decay width or cross-section for the reverse reaction is known.

Overall, this work profits from the previous works on the topic and uses them to extend the hadronic transport approach SMASH to treat multi-particle reactions. The most prominent multi-particle results in the literature apply 3 -to-2 reactions $[126,128,111]$, which are also introduced in SMASH. Besides treating 3-body reactions, the stochastic approach is extended in this work to include 5-body reactions for the first time. The extension is part of the now following derivation of stochastical rates.

\subsection{Theory of stochastic rates}

\subsubsection{Probability of a collision}

The particle density distribution function (or also referred to as probability density function) $f=f(\mathbf{r}, \mathbf{p}, t)$ gives the number of particles $N$ in the given phase-space interval $d^{3} r \frac{d^{3} p}{(2 \pi)^{3}}$ at $(\mathbf{r}, \mathbf{p})$ for time $t$ as

$$
d N=f(\mathbf{r}, \mathbf{p}, t) d^{3} r \frac{d^{3} p}{(2 \pi)^{3}}
$$

Note that $f(\mathbf{r}, \mathbf{p}, t)$ is the one-particle distribution function, which gives the number of particle for one particle species. For a mixture of different species, one-particle distribution function is needed for each of the species. The factor of $(2 \pi)^{3}$ is a choice of normalizing the 
phase space volume. The evolution of the particle density distribution function is given by the Boltzmann equation (Eq. 1.8) as discussed in Section 1.2.4.

The number of particles inside a timestep of $\Delta t$ changes when collisions occur, basically particles scattering in and out of the phase-space volume: $d N(t+\Delta t)-d N(t)=\Delta N_{\text {coll }}$. Where $\Delta N_{\text {coll }}$ inside $d^{3} r \frac{d^{3} p}{(2 \pi)^{3}}$ is given as

$$
\Delta N_{\text {coll }}=\left(\frac{\partial f}{\partial t}\right)_{\text {coll }} \Delta t d^{3} r \frac{d^{3} p}{(2 \pi)^{3}}
$$

where $\left(\frac{\partial f}{\partial t}\right)_{\text {coll }}$ is the referred to as the collision integral. The collision integral states the number of collisions per timestep per phase space volume.

The probability of a $12 \rightarrow 1^{\prime} 2^{\prime}$ collision between a (random) pair of particle 1 and particle 2 inside of $\Delta^{3} x$ during the next timestep $\Delta t$ (in $[t, t+\Delta t]$ ) if considering a random pair of particles inside the volume is given as the number of collisions between particles 1 and $2\left(\Delta N_{\text {coll }}^{12 \rightarrow 1^{\prime} 2^{\prime}}\right)$ over the number of all possible pairings of particles 1 and $2\left(\Delta N_{1} \Delta N_{2}\right)$ :

$$
P_{12 \rightarrow 1^{\prime} 2^{\prime}}=\frac{\Delta N_{\mathrm{coll}}^{12 \rightarrow 1^{\prime} 2^{\prime}}}{\Delta N_{1} \Delta N_{2}} .
$$

In preparation for employing the above numerically, the notation was already changed to signal that non-infinitesimal quantities will be considered, namely $d^{3} r \rightarrow \Delta^{3} x$ and therefore $d N \rightarrow \Delta N$. The proper solution is recovered for sufficient small volumes $\left(\Delta^{3} x \rightarrow\right.$ $0)$.

It is already in this most general form obvious that Eq. 4.3 is straight-forward to generalize for any type of $n \rightarrow m^{\prime}$ reaction with

$$
P_{n \rightarrow m^{\prime}}=\frac{\Delta N_{\text {coll }}^{n \rightarrow m^{\prime}}}{\Delta N_{1} \Delta N_{2} \ldots \Delta N_{n}} .
$$

In principle, all ingredients are listed with the above. The next step is to insert Eq. 4.2 with the collision integral and Eq. 4.1 into Eq. 4.3 and Eq. 4.4. The challenge that needs to be solved is that the collision integral is not computable in all cases.

\subsubsection{The collision integral}

\section{Derivation and Stosszahlansatz}

In the following, the derivation of the collision integral is discussed since it forms the basis of the following equations of the collision probability. The derivation follows the one presented in [297] and follows Boltzmann's original arguments and assumptions commonly referred to as the Stosszahlansatz.

The goal is to obtain an expression for $\Delta N_{\text {coll }} / \Delta t$, which is the change of particle number with momentum $p_{1}$ inside the volume $d^{3} x \frac{d^{3} p_{1}}{(2 \pi)^{3}}$. Note that the non-relativistic case for the derivation arguments is considered, but full relativistic equations will be eventually implemented.

The change of particle number can be split into a gain and a loss term to signify that particles scatter into and out of the phase-space volume.

$$
\frac{\Delta N_{\mathrm{coll}}}{\Delta t}=\frac{\Delta N^{+}}{\Delta t}-\frac{\Delta N^{-}}{\Delta t} .
$$


The assumption referred to as the Stosszahlansatz are the following.

1. Only binary collisions are considered. Note that this assumption is only employed for the derivation here. The extension of the derived collision integral to account for any reactions is given in the next section.

2. The momenta $p_{1}$ and $p_{2}$ of the two scattering partners before the collision are not correlated (so called molecular chaos).

3. The distribution function $f$ can be assumed constant over the time and range of the collision. Or in other words, the mean free path is longer than the collision range. The collision is short-ranged.

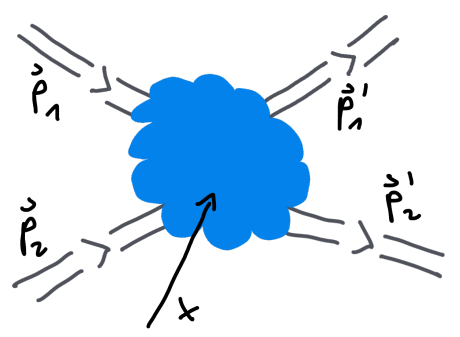

Figure 4.1: Binary collisions of two beams of particles with $v_{1}$ and $v_{2}$ (where $v_{i}=c p_{i} / E_{i}$ )

To find the collision number, a binary collision of two beams of particles with $v_{1}$ and $v_{2}$ (where $v_{i}=c p_{i} / E_{i}$ ) is explored (see Figure 4.1). The particle density of both particles is denoted as $d n_{i}=f_{i} \frac{d^{3} p}{(2 \pi)^{3}}$.

Without loss of generality, the case of one of the scattering beams at rest is examined: $v_{1}=0$. The total number of particles 1 at $x$ is given by multiplying the momentum density with $d^{3} x$ (see Eq. 4.1). The number of particles 2 colliding with the particles 1 in the time $\Delta t$ are all in a cylindrical volume $\left(V_{z y l}\right.$, illustrated in Figure 4.2). The base of the volume

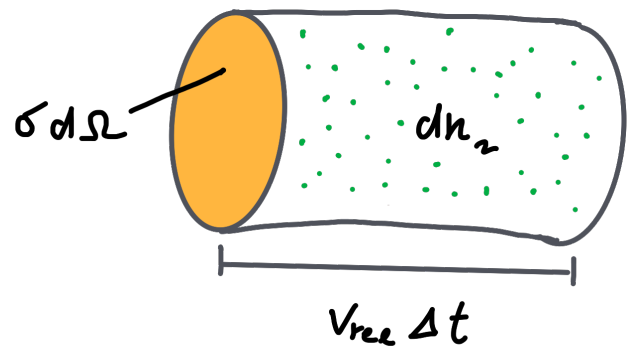

Figure 4.2: The "collision cylinder" (adapted from [297])

is the plane in which the particle scatterings are happening, which is given by an angle element $d \Omega$ multiplied by differential cross-section, noted as $\sigma_{d \Omega}$ here. The height of the cylinder in the rest frame of particles 1 is given by $v_{r e l} \Delta t$. The total number of particles 
2 that thereby pass by particles 1 is $d N_{2}=d n_{2} V_{z y l}=d n_{2} \sigma_{d \Omega} d \Omega v_{r e l} \Delta t$. The total number of collisions is then given as

$$
d N_{\text {coll }}=d N_{1} d N_{2}=d n_{1} d^{3} x d n_{2} \sigma_{d \Omega} d \Omega v_{r e l} \Delta t .
$$

Inserting the definition for $d n$, the equation reads

$$
d N_{\text {coll }}=f_{1} \frac{d^{3} p_{1}}{(2 \pi)^{3}} d^{3} x f_{2} \frac{d^{3} p_{2}}{(2 \pi)^{3}} \sigma_{d \Omega} d \Omega v_{r e l} \Delta t
$$

which results in the number of collisions performed within $d^{3} r d^{3} p_{1}$, if the integration of $d^{3} p_{2}$ and $d \Omega$ is performed. The number of collisions equals the loss of particles 1 , therefore

$$
\Delta N^{-}=\int_{\Omega} \int_{p_{2}} f_{1} f_{2} v_{r e l} \sigma_{d \Omega} \frac{d^{3} p_{1}}{(2 \pi)^{3}} d^{3} x \frac{d^{3} p_{2}}{(2 \pi)^{3}} d \Omega \Delta t
$$

The general definition of the differential cross-section in the PDG [256] for the binary case is

$$
\sigma_{d \Omega}=\frac{1}{4 E_{1} E_{2} v_{r e l}} \frac{d^{3} p_{1}^{\prime}}{(2 \pi)^{3} 2 E_{1}^{\prime}} \frac{d^{3} p_{2}^{\prime}}{(2 \pi)^{3} 2 E_{2}^{\prime}} \frac{1}{S_{1^{\prime} 2^{\prime}}} \overline{\left|\mathcal{M}_{12 \rightarrow 1^{\prime} 2^{\prime}}\right|^{2}}(2 \pi)^{4} \delta^{(4)}\left(P_{1}+P_{2}-P_{1}^{\prime}-P_{2}^{\prime}\right) .
$$

More explanations, including details on the matrix element $\overline{\left|\mathcal{M}_{12 \rightarrow 1^{\prime} 2^{\prime}}\right|^{2}}$ are given in the following sections.

Inserting the cross-section definition into the loss term of $\Delta N / \Delta t$ gives

$$
\begin{aligned}
\frac{\Delta N^{-}}{\Delta t}=\frac{1}{2 E_{1}} \int \frac{d^{3} p_{2}}{2 E_{2}(2 \pi)^{3}} & \frac{1}{S_{1^{\prime} 2^{\prime}}} \int \frac{d^{3} p_{1}^{\prime}}{(2 \pi)^{3} 2 E_{1}^{\prime}} \frac{d^{3} p_{2}^{\prime}}{(2 \pi)^{3} 2 E_{2}^{\prime}} f_{1} f_{2} \\
& \times \overline{\left|\mathcal{M}_{12 \rightarrow 1^{\prime} 2^{\prime}}\right|^{2}}(2 \pi)^{4} \delta^{(4)}\left(P_{1}+P_{2}-P_{1}^{\prime}-P_{2}^{\prime}\right) \frac{d^{3} p_{1}}{(2 \pi)^{3}} d^{3} x .
\end{aligned}
$$

Note that even though the necessary symmetry factor $S_{1^{\prime} 2^{\prime}}$ is already included here for consistency, a corresponding explanation is given in the next section.

The gain term $\frac{\Delta N^{+}}{\Delta t}$ is obtained by following the same arguments for two particle beams colliding with $v_{1}^{\prime}$ and $v_{2}^{\prime}$ scattering within $d^{3} x^{\prime} d^{3} p_{1}^{\prime}$ into $d^{3} x d^{3} p_{1}$. Therefore, the final expression for the collision integral for binary collision is identified as

$$
\left(\frac{\partial f}{\partial t}\right)_{\text {coll }}=\int \frac{d^{3} p_{2}}{(2 \pi)^{3}}\left(f_{1}^{\prime} f_{2}^{\prime}-f_{1} f_{2}\right) v_{r e l} \int d \sigma
$$

with the cross-section integration abbreviated to $\int d \sigma$ for readability.

Note that modifications accounting for the statistics of bosons and fermions were neglected in this derivation and the following. The reader is referred to [297] for further details. The quantum extension of the collision integral is the difference between the Boltzmann equation and its quantum analog, the Boltzmann-Uehling-Uhlenbeck (BUU) equation. The collision integral of the BUU equation is quoted in Eq. 2.26 as part of the discussion on Pauli blocking ${ }^{1}$.

\footnotetext{
${ }^{1}$ All results in this work do not include Pauli blocking.
} 


\section{General form}

The collision integral has generally gain and loss terms for every reaction that can occur. The two terms account for particles scattering into and out of the phase space volume.

As a first example, the integral derived in the previous section for 2-to-2 reactions only is given in full. To introduce some bookkeeping: The reaction indices are $12 \rightarrow 1^{\prime} 2^{\prime}$, so outgoing particle quantities have primed indices. And the equation focuses specifically on scatterings of particle 1 (with particle 2), which is possible since looking at one particle already gives us the number of collisions (they are the same for both). The change of particles, i.e. number of collisions, for the reaction $12 \leftrightarrow 1^{\prime} 2^{\prime}$ for particle species 1 is given by

$$
\begin{aligned}
& \left(\frac{\partial f}{\partial t}\right)_{\text {coll }}^{12 \leftrightarrow 1^{\prime} 2^{\prime}}=\frac{1}{2 E_{1}} \int \frac{d^{3} p_{2}}{(2 \pi)^{3} 2 E_{2}} \frac{1}{S_{1^{\prime} 2^{\prime}}} \int \frac{d^{3} p_{1}^{\prime}}{(2 \pi)^{3} 2 E_{1}^{\prime}} \frac{d^{3} p_{2}^{\prime}}{(2 \pi)^{3} 2 E_{2}^{\prime}} f_{1}^{\prime} f_{2}^{\prime} \\
& \times \overline{\left|\mathcal{M}_{1^{\prime} 2^{\prime} \rightarrow 12}\right|^{2}}(2 \pi)^{4} \delta^{(4)}\left(P_{1}^{\prime}+P_{2}^{\prime}-P_{1}-P_{2}\right) \\
& -\frac{1}{2 E_{1}} \int \frac{d^{3} p_{2}}{(2 \pi)^{3} 2 E_{2}} \frac{1}{S_{1^{\prime} 2^{\prime}}} \int \frac{d^{3} p_{1}^{\prime}}{(2 \pi)^{3} 2 E_{1}^{\prime}} \frac{d^{3} p_{2}^{\prime}}{(2 \pi)^{3} 2 E_{2}^{\prime}} f_{1} f_{2} \\
& \times \overline{\left|\mathcal{M}_{12 \rightarrow 1^{\prime} 2^{\prime}}\right|^{2}}(2 \pi)^{4} \delta^{(4)}\left(P_{1}+P_{2}-P_{1}^{\prime}-P_{2}^{\prime}\right)
\end{aligned}
$$

This is noted relativistically, with gain and loss term and integrated over the whole possible momentum space of particle 2. $\overline{|\mathcal{M}|^{2}}$ is the matrix element squared and averaged over the internal degeneracy (e.g. spin states) of the initial particle and summed over the internal degeneracy of the final particles for the given reaction.

The factor $S_{1^{\prime} 2^{\prime}}$ marks the necessary symmetry factors that need to be considered to avoid double counting in the phase space integration when $1^{\prime}$ and $2^{\prime}$ are identical i.e. the number of indistinguishable states. In general and in the following $S$ is therefore defined as $S=N_{i d}$ !, where $N_{i d}$ is the number identical particles in the state. So, for $12 \leftrightarrow 1^{\prime} 2^{\prime}$ reactions with $1^{\prime}$ and $2^{\prime}$ being identical, $S_{1^{\prime} 2^{\prime}}=2 !=2$.

To write the collision integral in a more compact form, the following abbreviations are used in this chapter:

$$
d \Gamma=\frac{d^{3} p}{(2 \pi)^{3} 2 E}
$$

for stable particles with $E^{2}=p^{2}+m^{2}$. For resonances with a general spectral function the form is given as

$$
d \Gamma=\frac{d^{4} p}{(2 \pi)^{4}} \frac{\pi}{\sqrt{s}} \mathcal{A}(\sqrt{s})=\frac{d M}{2} \frac{1}{E_{M}} \frac{d^{3} p}{(2 \pi)^{3}} \mathcal{A}(M),
$$

where $M=\sqrt{s}=\sqrt{p^{0,2}-p^{2}}$ the mass of the resonances, $E_{M}^{2}=p^{2}+M^{2}$ and $\mathcal{A}(M)$ as the spectral function. The $d \Gamma_{k}$ for stable particles is recovered when considering a $\delta$ spectral function $\mathcal{A}(M)=2 M \delta\left(M^{2}-m^{2}\right)$ for such particles.

The whole differential n-body phase space is shortened to

$$
d \Phi_{n}=(2 \pi)^{4} \delta^{(4)}\left(P-\sum_{k=1}^{n} p_{k}\right) \prod_{k=1}^{n} d \Gamma_{k},
$$


where $P$ is total 4 -momentum of the reaction. With those abbreviations, the collision integral reads

$$
\begin{aligned}
\left(\frac{\partial f}{\partial t}\right)_{\text {coll }}^{12 \leftrightarrow 1^{\prime} 2^{\prime}} & =\frac{1}{2 E_{1}} \int d \Gamma_{2} \frac{1}{S_{1^{\prime} 2^{\prime}}} \int f_{1}^{\prime} f_{2}^{\prime} \overline{\left|\mathcal{M}_{1^{\prime} 2^{\prime} \rightarrow 12}\right|^{2}} d \Phi_{2^{\prime}} \\
& -\frac{1}{2 E_{1}} \int d \Gamma_{2} \frac{1}{S_{1^{\prime} 2^{\prime}}} \int f_{1} f_{2} \overline{\left|\mathcal{M}_{12 \rightarrow 1^{\prime} 2^{\prime}}\right|^{2}} d \Phi_{2^{\prime}} .
\end{aligned}
$$

As mentioned, the collision integral has terms for all possible reactions. The gain and loss terms for a generic $2 \rightarrow m$ reaction is as follows

$$
\begin{aligned}
\left(\frac{\partial f}{\partial t}\right)_{\text {coll }}^{12 \leftrightarrow 1^{\prime} 2^{\prime} \ldots m^{\prime}} & =\frac{1}{2 E_{1}} \int d \Gamma_{2} \mathcal{S}^{\dagger} \int f_{1}^{\prime} f_{2}^{\prime} \ldots f_{m}^{\prime} \overline{\left|\mathcal{M}_{1^{\prime} 2^{\prime} \ldots m^{\prime} \rightarrow 12}\right|^{2}} d \Phi_{m^{\prime}} \\
& +\frac{1}{2 E_{1}} \int \prod_{k=2}^{m} d \Gamma_{k} \mathcal{S}^{\dagger} \int f_{1}^{\prime} f_{2}^{\prime} \overline{\left|\mathcal{M}_{1^{\prime} 2^{\prime} \rightarrow 12 \ldots m}\right|^{2}} d \Phi_{2^{\prime}} \\
& -\frac{1}{2 E_{1}} \int d \Gamma_{2} \mathcal{S}^{\dagger} \int f_{1} f_{2} \overline{\left|\mathcal{M}_{12 \rightarrow 1^{\prime} 2^{\prime} \ldots m^{\prime}}\right|^{2}} d \Phi_{m^{\prime}} \\
& -\frac{1}{2 E_{1}} \int \prod_{k=2}^{m} d \Gamma_{k} \mathcal{S}^{\dagger} \int f_{1} f_{2} \ldots f_{m} \overline{\left|\mathcal{M}_{12 \ldots m \rightarrow 1^{\prime} 2^{\prime}}\right|^{2}} d \Phi_{2^{\prime}}
\end{aligned}
$$

Here, the gain (loss) term have 2 terms each, since the particle can be gained (lost) either by $2 \rightarrow m$ or $m \rightarrow 2$, as it assumed here that particle $\mathbf{1}$ is part of both sides of the reaction ${ }^{2}$. Note that the necessary symmetry factors are abbreviated here with the place holder $\mathcal{S}^{\dagger}$. Finally, in its most generic form, the collision integral for a $n \rightarrow m$ reaction is

$$
\begin{aligned}
\left(\frac{\partial f}{\partial t}\right)_{\text {coll }}^{12 \ldots n \leftrightarrow 1^{\prime} 2^{\prime} \ldots m^{\prime}} & =\frac{1}{2 E_{1}} \int \prod_{k=2}^{n} d \Gamma_{k} \mathcal{S}^{\dagger} \int f_{1}^{\prime} f_{2}^{\prime} \ldots f_{m}^{\prime} \overline{\left|\mathcal{M}_{1^{\prime} 2^{\prime} \ldots m^{\prime} \rightarrow 12 \ldots n}\right|^{2}} d \Phi_{m^{\prime}} \\
& +\frac{1}{2 E_{1}} \int \prod_{k=2}^{m} d \Gamma_{k} \mathcal{S}^{\dagger} \int f_{1}^{\prime} f_{2}^{\prime} \ldots f_{n}^{\prime} \overline{\left|\mathcal{M}_{1^{\prime} 2^{\prime} \ldots n^{\prime} \rightarrow 12 \ldots m}\right|^{2}} d \Phi_{n^{\prime}} \\
& -\frac{1}{2 E_{1}} \int \prod_{k=2}^{n} d \Gamma_{k} \mathcal{S}^{\dagger} \int f_{1} f_{2} \ldots f_{n} \overline{\left|\mathcal{M}_{12 \ldots n \rightarrow 1^{\prime} 2^{\prime} \ldots m^{\prime}}\right|^{2}} d \Phi_{m^{\prime}} \\
& -\frac{1}{2 E_{1}} \int \prod_{k=2}^{m} d \Gamma_{k} \mathcal{S}^{\dagger} \int f_{1} f_{2} \ldots f_{m} \overline{\left|\mathcal{M}_{12 \ldots m \rightarrow 1^{\prime} 2^{\prime} \ldots n}\right|^{2}} d \Phi_{n^{\prime}}
\end{aligned}
$$

Note that for the general cases, the assumption of binary collisions of the Stosszahlansatz is lifted.

\subsubsection{Probability for 2-to-2 reactions}

Since the two-body scattering case was historically the first one discussed in the literature [134], the discussion for concrete reaction classes starts with this case. The relevant part of the collision integral is the loss term, since only the probability within the phase space

\footnotetext{
${ }^{2}$ The same is also true, if all particles are identical. This is the most extensive case for the collision integral, since, if neither particle $\mathbf{1}$ is appearing on both sides of the reaction nor the particles are identical, the collision term collapses to only 1 term for gain and loss term for the $2 \rightarrow m$ reaction.
} 
volume is considered (the gain term involves scatterings into the phase space volume from outside). The (absolute value of the) loss term is noted differentially in $\Delta^{3} p_{2}$, since for the probability in the end shall be given for two particle with momenta inside $\Delta^{3} p_{1}$ and $\Delta^{3} p_{2}$ and within the same volume $\Delta^{3} x$. Using Eq. 4.2 and the loss term from Eq. 4.12 and refraining from using the above introduced abbreviations for this first example:

$$
\begin{aligned}
\frac{\Delta N_{\mathrm{coll}}^{12 \rightarrow 1^{\prime} 2^{\prime}}}{\Delta t \Delta^{3} x \Delta^{3} p_{1} /(2 \pi)^{3}}=\frac{1}{2 E_{1}} & \frac{\Delta^{3} p_{2}}{(2 \pi)^{3} 2 E_{2}} \frac{1}{S_{1^{\prime} 2^{\prime}}} \int \frac{d^{3} p_{1}^{\prime}}{(2 \pi)^{3} 2 E_{1}^{\prime}} \frac{d^{3} p_{2}^{\prime}}{(2 \pi)^{3} 2 E_{2}^{\prime}} f_{1} f_{2} \\
& \times\left|\mathcal{M}_{12 \rightarrow 1^{\prime} 2^{\prime}}\right|^{2}(2 \pi)^{4} \delta^{(4)}\left(P_{1}+P_{2}-P_{1}^{\prime}-P_{2}^{\prime}\right)
\end{aligned}
$$

This expression simplifies, since the cross-section appears.

$$
\begin{aligned}
\sigma_{12 \rightarrow 1^{\prime} 2^{\prime}} & =\frac{1}{2 E_{1}} \frac{1}{2 E_{2}} \frac{1}{v_{r e l}} \frac{1}{S_{1^{\prime} 2^{\prime}}} \int \frac{d^{3} p_{1}^{\prime}}{(2 \pi)^{3} 2 E_{1}^{\prime}} \frac{d^{3} p_{2}^{\prime}}{(2 \pi)^{3} 2 E_{2}^{\prime}} \\
& \times \overline{\left|\mathcal{M}_{12 \rightarrow 1^{\prime} 2^{\prime}}\right|^{2}}(2 \pi)^{4} \delta^{(4)}\left(P_{1}+P_{2}-P_{1}^{\prime}-P_{2}^{\prime}\right),
\end{aligned}
$$

Note that this identification is possible, since $f_{1} f_{2}$ do not depend on $p_{i}^{\prime}$. $v_{r e l}$ is the relative velocity is defined as

$$
\begin{aligned}
v_{r e l} & =\frac{\sqrt{\left(p_{1} \cdot p_{2}\right)^{2}-m_{1}^{2} m_{2}^{2}}}{E_{1} E_{2}} \\
& =\frac{\sqrt{\left(s-m_{1}^{2}-m_{2}^{2}\right)^{2}-m_{1}^{2} m_{2}^{2}}}{2 E_{1} E_{2}} \\
& =\frac{\lambda^{1 / 2}\left(s, m_{1}^{2}, m_{2}^{2}\right)}{2 E_{1} E_{2}},
\end{aligned}
$$

Sometimes this is also called Møller velocity. $\lambda$ is the so called Källén function and defined as

$$
\lambda(a, b, c)=(a-b-c)^{2}-4 b c .
$$

Recalling the definition for the particle density distribution function (Eq. 4.1):

$$
f_{i}=\frac{\Delta N_{i}}{\Delta^{3} x_{i}} \Delta^{3} p_{i} /(2 \pi)^{3} .
$$

This definition is inserted into Eq. 4.19 and results in the following.

$$
\begin{array}{r}
\frac{\Delta N_{\text {coll }}^{12-1^{\prime} 2^{\prime}}}{\Delta t \Delta^{3} x \Delta^{3} p_{1} /(2 \pi)^{3}}=\frac{1}{2 E_{1}} \frac{\Delta^{3} p_{2}}{(2 \pi)^{3} 2 E_{2}} f_{1} f_{2} 2 E_{1} 2 E_{2} v_{r e l} \sigma_{12 \rightarrow 1^{\prime} 2^{\prime}} \\
=\frac{1}{2 E_{1}} \frac{\Delta^{3} p_{2}}{(2 \pi)^{3} 2 E_{2}} \frac{\Delta N_{1}}{\Delta^{3} x \Delta^{3} p_{1} /(2 \pi)^{3}} \frac{\Delta N_{2}}{\Delta^{3} x \Delta^{3} p_{2} /(2 \pi)^{3}} 2 E_{1} 2 E_{2} v_{r e l} \sigma_{12 \rightarrow 1^{\prime} 2^{\prime}}
\end{array}
$$

Note that as $\Delta^{3} x_{i}$ is the same for all particles, its index is dropped.After reducing and rearranging according to the definition of the probability in Eq. 4.3, the probability for 2 -to-2 reactions known from the literature is obtained.

$$
P_{12 \rightarrow 1^{\prime} 2^{\prime}}=\frac{\Delta N_{\mathrm{coll}}^{12 \rightarrow 1^{\prime} 2^{\prime}}}{\Delta N_{1} \Delta N_{2}}=\frac{\Delta t}{\Delta^{3} x} v_{r e l} \sigma_{12 \rightarrow 1^{\prime} 2^{\prime}}
$$

As long as the cross-section for the specific $12 \rightarrow 1^{\prime} 2^{\prime}$ process is known, the probability is therefore straight-forward to calculate. Furthermore, it is clear from the expression that the probability is inherently boost invariant. 


\subsubsection{Probability for 2-to-m reactions}

The cross-section is not only defined for a 2-body scattering with a 2-body final state. In its most general form it is given for an m-body final state (see for example [256]):

$$
\sigma_{12 \rightarrow 1^{\prime} 2^{\prime} \ldots m^{\prime}}=\frac{1}{2 E_{1}} \frac{1}{2 E_{2}} \frac{1}{v_{r e l}} \frac{1}{S_{1^{\prime} 2^{\prime} \ldots m^{\prime}}} \int \overline{\left|\mathcal{M}_{12 \rightarrow 1^{\prime} 2^{\prime} \ldots m^{\prime}}\right|^{2}} d \Phi_{m^{\prime}}
$$

Comparing this expression with the relevant (third) term of the collision integral $\left(\frac{\partial f}{\partial t}\right)_{\text {coll }}^{12 \leftrightarrow 1^{\prime} 2^{\prime} \ldots m^{\prime}}$ in Eq. 4.17, one again identifies the cross-section. The rest of the calculation is analogue to the 2-to-2 case discussed in the previous section. The collision probability obtained reads as follows,

$$
P_{12 \rightarrow 1^{\prime} 2^{\prime} \ldots m^{\prime}}=\frac{\Delta N_{\mathrm{coll}}^{12 \rightarrow 1^{\prime} 2^{\prime} \ldots m^{\prime}}}{\Delta N_{1} \Delta N_{2}}=\frac{\Delta t}{\Delta^{3} x} v_{r e l} \sigma_{12 \rightarrow 1^{\prime} 2^{\prime} \ldots m^{\prime}} .
$$

Again this probability is straight-forward to calculate if the cross-section is known for the specific process. It is important to stress here that 2-to-m processes are straight-forward to treat with a geometric collision criterion. For all 2-body scattering processes where a cross-section is defined, a geometric interpretation of it and, therefore, a distance-based collision criterion is possible. The challenge is to treat the back-reaction $(m \rightarrow 2)$ for $m>2$ in order to obtain detailed balance. As long as 2-body scattering processes are discussed, the stochastic collision criterion offers an alternative but ultimately equivalent treatment (leaving the aspect of invariance under Lorentz transformations aside).

\subsubsection{Probability for $\mathrm{n}$-to-m reactions}

The real advantage of the stochastic collision criterion is that it is straight-forward to apply to n-to-m reactions with $n>2$ and/or $m>2$. This requires a general formulation of the probability. The loss rate of particle 1 reads,

$$
\frac{\Delta N_{\text {coll }}^{12 \ldots n \rightarrow 1^{\prime} 2^{\prime} \ldots m^{\prime}}}{\Delta t \Delta^{3} x \Delta^{3} p_{1} /(2 \pi)^{3}}=\left|\frac{\partial f}{\partial t}\right|_{\text {coll,loss }}^{12 \ldots n \rightarrow 1^{\prime} 2^{\prime} \ldots m^{\prime}} .
$$

Here the notation of the collision term signifies that the absolute value of the loss term is meant. Inserting the definition of the collision integral (differential in the initial state momenta) and writing the distribution function before the integral, the equation reads

$$
\begin{aligned}
\frac{\Delta N_{\text {coll }}^{12 \ldots} . n \rightarrow 1^{\prime} 2^{\prime} \ldots m^{\prime}}{\Delta t \Delta^{3} x \Delta^{3} p_{1} /(2 \pi)^{3}}=\frac{1}{2 E_{1}} & \prod_{j=2}^{n} \frac{\Delta^{3} p_{j}}{(2 \pi)^{3} 2 E_{j}} f_{1} \prod_{k=2}^{n} f_{k} \frac{1}{S_{1^{\prime} 2^{\prime} \ldots m^{\prime}}} \\
& \times \int \overline{\left|\mathcal{M}_{12 \ldots n \rightarrow 1^{\prime} 2^{\prime} \ldots m^{\prime}}\right|^{2}} d \Phi_{m^{\prime}}
\end{aligned}
$$

The remaining integral is abbreviated with

$$
I_{n m}^{\prime}=\frac{1}{S_{1^{\prime} 2^{\prime} \ldots m^{\prime}}} \int \overline{\left|\mathcal{M}_{12 \ldots n \rightarrow 1^{\prime} 2^{\prime} \ldots m^{\prime}}\right|^{2}} d \Phi_{m^{\prime}} .
$$

Using the definition of the distribution function in Eq. 4.1 and the abbreviation, the equation reads

$$
\frac{\Delta N_{\mathrm{coll}}^{12 \ldots . . n \rightarrow 1^{\prime} 2^{\prime} \ldots m^{\prime}}}{\Delta t \Delta^{3} x \frac{\Delta^{3} p_{1}}{(2 \pi)^{3}}}=\frac{1}{2 E_{1}} \prod_{j=2}^{n} \frac{\Delta^{3} p_{j}}{(2 \pi)^{3} 2 E_{j}} \frac{\Delta N_{1}}{\Delta^{3} x \frac{\Delta^{3} p_{1}}{(2 \pi)^{3}}} \prod_{k=2}^{n} \frac{\Delta N_{k}}{\Delta^{3} x \frac{\Delta^{3} p_{k}}{(2 \pi)^{3}}} I_{n m}^{\prime} .
$$


The probability for a n-to-m reaction is finally obtained by recalling Eq. 4.4 and rearranging:

$$
P_{12 \ldots n \rightarrow 1^{\prime} 2^{\prime} \ldots m^{\prime}}=\frac{\Delta N_{\text {coll }}^{12 \ldots n \rightarrow 1^{\prime} 2^{\prime} \ldots m^{\prime}}}{\prod_{j=1}^{n} \Delta N_{j}}=\frac{1}{2^{n} \prod_{j=1}^{n} E_{j}} I_{n m}^{\prime} \frac{\Delta t}{\left(\Delta^{3} x\right)^{n-1}} .
$$

This represents the most general definition of the collision probability of the stochastic criterion. However, note that this definition is not applicable for most cases since the matrix element in the $I_{m n}$ integral is generally unknown, in particular for multiparticle processes. But, for $n \rightarrow m$ processes where the cross-section or decay width of the reverse $m \rightarrow n$ process is known $(m=1,2)$, this issue can be resolved as described in the following sections for several reaction classes.

\subsubsection{Test-particle method with stochastic rates}

Transport approaches effectively solve the Boltzmann equation by sampling the distribution density function $f(\mathbf{r}, \mathbf{p}, t)$ with single particles that have a fixed $\mathbf{r}$ and $\mathbf{p}$ for a given $t$. To represent and ultimately evolve a smooth distribution density, many point-like particles are needed. Therefore it is a common practice to increase (oversample) the number of particles by a factor $N_{\text {test }}$ (test particle number). Since the density increases accordingly, the number of interactions would also artificially be increased and the results would change. Therefore the probability of a collision has to be lowered at the same time.

To determine how the probability has to be scaled with the test particle number, first the scaling for the particle amount $(\Delta N)$ and the collision number $\left(\Delta N_{\text {coll }}\right)$ is given

$$
\begin{aligned}
\Delta N & \rightarrow N_{\text {test }} \Delta N \\
\Delta N_{\text {coll }} & \rightarrow N_{\text {test }} \Delta N_{\text {coll }},
\end{aligned}
$$

so both scale linearly with the the number of test particles. Recalling Eq. 4.4, the scaled probability $P_{n \rightarrow m}^{\prime}$ is therefore given as

$$
P_{n \rightarrow m}^{\prime}=\frac{N_{\text {test }} \Delta N_{n \rightarrow m}}{N_{\text {test }}^{n} \Delta N_{1} \Delta N_{2} \ldots \Delta N_{n}}=P_{n \rightarrow m} \frac{1}{N_{\text {test }}^{n-1}} .
$$

When employing the test-particle method, the probability has to be scaled with $\frac{1}{N_{\text {test }}^{n-1}}$. This scaling matches the results reported in the literature of $\frac{1}{N_{\text {test }}}$ for 2-body scattering in [134] and $\frac{1}{N_{\text {test }}^{2}}$ for 3-body scattering in [126, 217].

\subsubsection{Phase space integrals}

In the following sections, the integrated m-body phase space appears in equations for the probability. Therefore its form for the relevant cases is discussed in this section as a prerequisite. The integration appears for the final state in the integral $I_{n m}$ (Eq. 4.31 and Eq.4.32), because it is assumed that the matrix element does not depend on the final state momenta as an approximation. So the integrations left to calculate are of the form

$$
\Phi_{m}=\int d \Phi_{m} .
$$

Note that the phase space integrals (except for the one-body case) assume the particles to be stable i.e. have $\delta$-like, infinitely narrow spectral functions $\mathcal{A}(M)=2 M \delta\left(M^{2}-m^{2}\right)$, so $d \Gamma=\frac{d^{3} p}{(2 \pi)^{3} 2 E}$, which is the relevant case for this work. See Section4.1.10 for a discussion on the two- or more-body phase space involving resonances. 


\section{One-body phase space}

The phase space for one-body resonances is trivially obtained when plugging in $d \Gamma$ as given in Eq. 4.1.2 for resonances.

$$
\Phi_{1}=\int d \Gamma_{1}(2 \pi)^{4} \delta^{(4)}\left(P-P_{1}\right)=\int \frac{d^{4} p_{1}}{(2 \pi)^{4}} \frac{\pi}{m} \mathcal{A}(m)(2 \pi)^{4} \delta^{(4)}\left(P-P_{1}\right)=\frac{\pi}{\sqrt{s}} \mathcal{A}(\sqrt{s})
$$

\section{Two-body phase space}

For two particles, the phase space is given as

$$
\Phi_{2}\left(s, m_{1}, m_{2}\right)=\int d \Gamma_{1} d \Gamma_{2}(2 \pi)^{4} \delta^{(4)}\left(P-P_{1}-P_{2}\right)=\frac{\lambda^{1 / 2}\left(s, m_{1}^{2}, m_{2}^{2}\right)}{8 \pi s},
$$

which is still easily obtained with $\lambda$ defined as in Eq. 4.22 .

\section{Three-body phase space}

The definition for the three-body phase space is

$$
\Phi_{3}=\int d \Gamma_{1} d \Gamma_{2} d \Gamma_{3}(2 \pi)^{4} \delta^{(4)}\left(P-P_{1}-P_{2}-P_{3}\right)
$$

This is reformulated for stable particles with the definitions employed in the PDG Book for the three-body decay width [256], which is employed to create Dalitz plots (= scatter plot in $m_{12}^{2}$ and $\left.m_{23}^{2}\right)$ :

$$
d \Gamma_{1 \rightarrow 3}=\frac{1}{2 M} \overline{\left|\mathcal{M}_{1 \rightarrow 3}\right|^{2}} d \Phi_{3}=\frac{1}{(2 \pi)^{3}} \frac{1}{32 M^{3}} \overline{\left|\mathcal{M}_{1 \rightarrow 3}\right|^{2}} d m_{12}^{2} d m_{23}^{2},
$$

where $d m_{i j}^{2}=\left(P_{i}+P_{j}\right)^{2}$ and $M^{2}=s$ the squared mass of the resonance. The phase space integration is rewritten as

$$
\Phi_{3}=\frac{1}{(2 \pi)^{3}} \frac{1}{16 M^{2}} I_{3}
$$

with the integral $I_{3}$ defined as

$$
I_{3} \equiv \int d m_{12}^{2} d m_{23}^{2}=\int_{\left(m_{1}+m_{2}\right)^{2}}^{\left(M-m_{3}\right)^{2}}\left[\left(m_{23, \text { max }}^{2}-m_{23, \min }^{2}\right)\right] d m_{12}^{2} .
$$

The min and max values are defined as in the PDG book as

$$
\begin{aligned}
& m_{23, \text { min }}^{2}=\left(E_{2}^{*}+E_{3}^{*}\right)^{2}-\left(\sqrt{E_{2}^{* 2}-m_{2}^{2}}+\sqrt{E_{3}^{* 2}-m_{3}^{2}}\right)^{2} \\
& m_{23, \text { max }}^{2}=\left(E_{2}^{*}+E_{3}^{*}\right)^{2}-\left(\sqrt{E_{2}^{* 2}-m_{2}^{2}}-\sqrt{E_{3}^{* 2}-m_{3}^{2}}\right)^{2}
\end{aligned}
$$

with $E_{2}^{*}=\left(m_{12}^{2}-m_{1}^{2}+m_{2}^{2}\right) / 2 m_{12}$ and $E_{3}^{*}=\left(M^{2}-m_{12}^{2}-m_{3}^{2}\right) / 2 m_{12}$.

For example, for the $\omega \rightarrow 3 \pi$ decay (for $\omega$ with pole mass) this results in $I_{3}=0.07514$ $\mathrm{GeV}^{4}$. For the massless case $m_{1}=m_{2}=m_{3}=0$ a simple analytical from is obtained [298]:

$$
\Phi_{3}=\frac{1}{(2 \pi)^{5}} \frac{\pi^{2} M^{2}}{8}
$$


which gives $I_{3}$ in the massless case as

$$
I_{3}=\frac{M^{4}}{2}
$$

The massless case is in particular useful for cross-checking the numerical integration routines.

An analytical formula for the three-body phase space is given in Ref. [299]. For the case of three equal masses $\left(m_{e q}\right)$ that formula reduces to [300]

$$
\begin{aligned}
& \Phi_{3}\left(M^{2}\right)=\frac{1}{128 \pi^{3} M^{2}} \sqrt{\left(M-m_{e q}\right)\left(M+3 m_{e q}\right)} \\
& \left.\times\left[\frac{1}{2}\left(M-m_{e q}\right)\left(M^{2}+3 m_{e q}^{2}\right) E\left(k_{\mathrm{eq}}\right)-4 m_{e q}^{2} M K\left(k_{\mathrm{eq}}\right)\right]\right],
\end{aligned}
$$

with the complete elliptic integrals,

$$
\begin{aligned}
K(k) & =\int_{0}^{1} \frac{d t}{\sqrt{\left(1-t^{2}\right)\left(1-k^{2} t^{2}\right)}}, \\
E(k) & =\int_{0}^{1} d t \sqrt{\frac{1-k^{2} t^{2}}{1-t^{2}}}
\end{aligned}
$$

and

$$
k_{\mathrm{eq}}=\sqrt{\frac{\left(M+m_{e q}\right)^{3}\left(M-3 m_{e q}\right)}{\left(M-m_{e q}\right)^{3}\left(M+3 m_{e q}\right)}} .
$$

Furthermore, it is possible to write down a cyclic phase-space integration, where the mbody phase space is defined as an integration over the lower order phase space (see e.g. Appendix B in [129]). Employing this idea, the n-body phase space is calculated with

$$
\Phi_{n}=\int \frac{d s_{1}}{2 \pi} \frac{d s_{2}}{2 \pi} \Phi_{2}\left(s, s_{1}, s_{2}\right) \Phi_{k}\left(s_{1}, m_{1}^{2}, m_{2}^{2}, \ldots, m_{k}^{2}\right) \Phi_{n-k}\left(s_{2}, m_{k+1}^{2}, \ldots, m_{n}^{2}\right)
$$

For $n=3$, when assuming $\Phi_{1}=\frac{\pi}{\sqrt{s}} \mathcal{A}(\sqrt{s})=2 \pi \delta\left(s-m_{1}^{2}\right)$, this results in

$$
\Phi_{3}\left(s, m_{1}^{2}, m_{2}^{2}, m_{3}^{2}\right)=\frac{1}{2 \pi} \int_{\left(m_{1}+m_{2}\right)^{2}}^{\left(\sqrt{s}-m_{3}\right)^{2}} d s_{1} \Phi_{2}\left(s, s_{1}, m_{3}^{2}\right) \Phi_{2}\left(s_{1}, m_{1}^{2}, m_{2}^{2}\right) .
$$

\section{Five-body phase space}

The five-body phase space

$$
\Phi_{5}=\int d \Gamma_{1} d \Gamma_{2} d \Gamma_{3} d \Gamma_{4} d \Gamma_{5}(2 \pi)^{4} \delta^{(4)}\left(P-P_{1}-P_{2}-P_{3}-P_{4}-P_{5}\right)
$$

is even more tedious to calculate than the 3-body phase space (even for stable particles) and simple analytic forms are difficult to derive Therefore, the phase space needs to be sampled numerically. The numerical calculation can be based on the recursive definition of the n-body phase space given in Eq. 4.49. Together with the 3-body phase space (Eq. 4.50) the 5-body is given as

$$
\Phi_{5}\left(s, m_{1}^{2}, m_{2}^{2}, m_{3}^{2}, m_{4}^{2}, m_{5}^{2}\right)=\frac{1}{2 \pi} \int_{\left(m_{3}+m_{4}+m_{5}\right)^{2}}^{\left(\sqrt{s}-m_{1}-m_{2}\right)^{2}} d s_{1} \Phi_{3}\left(s, m_{1}^{2}, m_{2}^{2}, s_{1}\right) \Phi_{3}\left(s_{1}, m_{3}^{2}, m_{4}^{2}, m_{5}^{2}\right)
$$


with the massless case in [298] given as

$$
\Phi_{5}=\frac{s^{3}}{2(4 \pi)^{7} 4 ! 3 !} .
$$

The massless case is a particularly useful case to verify the numeric calculation. When employing the 5-body phase space for the massive case, it will be parametrized. See Section 4.3.3.

\subsubsection{Decays}

Even though the focus in this chapter is on scatterings of two or more particles, the same method of defining a probability is also valid for decays.

$$
P_{1 \rightarrow m}=\frac{\Delta N_{\mathrm{dec}}}{\Delta N_{R}},
$$

where $\Delta N_{R}$ is the particle number of the resonance $(R)$ that is decaying and $\Delta N_{\text {dec }}$ the number of decays, both inside the sub-volume $\Delta^{3} x$. The rate of decays is (see Eq. 4.28)

$$
\frac{\Delta N_{\mathrm{dec}}}{\Delta t}=\Delta^{3} x \frac{\Delta^{3} p_{R}}{(2 \pi)^{3}}\left|\frac{\partial f}{\partial t}\right|_{\text {coll,loss }}^{R \rightarrow 1^{\prime} 2^{\prime} \ldots m^{\prime}}
$$

The loss term for a $1 \rightarrow m$ decay is

$$
\left|\frac{\partial f}{\partial t}\right|_{\text {coll,loss }}^{R \rightarrow 1^{\prime} 2^{\prime} \ldots m^{\prime}}=\frac{1}{2 E_{R}} \frac{1}{S_{1^{\prime} 2^{\prime} \ldots m^{\prime}}} \int f_{R} \overline{\left|\mathcal{M}_{1 \rightarrow 1^{\prime} 2^{\prime} \ldots m^{\prime}}\right|^{2}} d \Phi_{m^{\prime}} .
$$

Inserting the loss term and decay rate into the decay probability in Eq. 4.54 results in

$$
P_{1 \rightarrow m}=\Delta t \frac{1}{2 E_{R}} \frac{1}{S_{1^{\prime} 2^{\prime} \ldots m^{\prime}}} \int \overline{\left|\mathcal{M}_{1 \rightarrow 1^{\prime} 2^{\prime} \ldots m^{\prime}}\right|^{2}} d \Phi_{m^{\prime}}
$$

where also (the non-infinitesimal version of) Eq. 4.1 is used for the distribution function:

$$
f_{R}=\frac{\Delta N_{R}}{\Delta^{3} x \frac{d^{3} p_{R}}{(2 \pi)^{3}}}
$$

In this formulation of the probability, the total decay rate can be identified

$$
\Gamma_{1 \rightarrow m}=\frac{1}{2 M} \frac{1}{S_{1^{\prime} 2^{\prime} \ldots m^{\prime}}} \int \overline{\left|\mathcal{M}_{1 \rightarrow 1^{\prime} 2^{\prime} \ldots m^{\prime}}\right|^{2}} d \Phi_{m^{\prime}},
$$

where $M$ is the (off-shell) mass of the resonance that is equal to the $\sqrt{s}$ of the final state. Two examples of decays considered in this work are $1 \rightarrow 2$ and $1 \rightarrow 3$ decays. The decay width in those cases reads

$$
\Gamma_{1 \rightarrow 2}=\frac{1}{2 M} \frac{1}{S_{12}} \int \overline{\left|\mathcal{M}_{1 \rightarrow 2}\right|^{2}} d \Gamma_{1} d \Gamma_{2}(2 \pi)^{4} \delta^{(4)}\left(P-p_{1}-p_{2}\right)
$$

and

$$
\Gamma_{1 \rightarrow 3}=\frac{1}{2 M} \frac{1}{S_{123}} \int \overline{\left|\mathcal{M}_{1 \rightarrow 3}\right|^{2}} d \Gamma_{1} d \Gamma_{2} d \Gamma_{3}(2 \pi)^{4} \delta^{(4)}\left(P-p_{1}-p_{2}-p_{3}\right)
$$


where the necessary symmetry factors are included that divide by the number of identical final states if identical particles are found in the final state. Note that here and in the following the primed notation is changed. Only quantities are noted as primed (to identify the final state), when it is not clear from the number of particles whether they belong to the initial or final states.

In the case of a constant i.e. independent from final momenta $\overline{|\mathcal{M}|^{2}}$ the total $1 \rightarrow 2$ decay rate is

$$
\Gamma_{1 \rightarrow 2}=\frac{1}{32 \pi^{2}} \frac{1}{S_{12}} \overline{\left|\mathcal{M}_{1 \rightarrow 2}\right|^{2}} \frac{\left|\mathbf{p}_{1}\right|}{M^{2}} 4 \pi,
$$

which is found in [256] for the decay rate in the center-of-mass frame (or rest frame of the resonance and with

$$
\left|\mathbf{p}_{1}\right|=\frac{\lambda^{1 / 2}\left(s, m_{1}^{\prime 2}, m_{2}^{\prime 2}\right)}{2 \sqrt{s}}, \quad \sqrt{s}=M
$$

where the definition of $\lambda$ is found in Eq. 4.22. The 2-body decay rate therefore is

$$
\Gamma_{1 \rightarrow 2}=\frac{1}{16 \pi} \frac{1}{S_{12}} \overline{\left|\mathcal{M}_{1 \rightarrow 2}\right|^{2}} \frac{\lambda^{1 / 2}\left(M^{2}, m_{1}^{\prime 2}, m_{2}^{\prime 2}\right)}{M^{3}}
$$

in the case of a constant matrix element. For $1 \rightarrow 3$ decay in this case, the decay rate reads

$$
\Gamma_{1 \rightarrow 3}=\frac{1}{2 M} \frac{1}{S_{123}} \overline{\left|\mathcal{M}_{1 \rightarrow 3}\right|^{2}} \Phi_{3}
$$

with the 3-body phase space integration $\Phi_{3}$ already discussed in Section 4.1.7 in detail.

Coming back to the probability, with the general definition of $\Gamma_{1 \rightarrow m}$ the decay probability reads

$$
P_{1 \rightarrow m}=\Delta t \frac{M}{E_{R}} \Gamma_{1 \rightarrow m},
$$

which simplifies in the rest frame $(\mathrm{RF})$ of the resonance $\left(M=E_{R}\right)$ to:

$$
P_{1 \rightarrow m}^{\mathrm{RF}}=\Delta t \Gamma_{1 \rightarrow m} .
$$

Note that this matches the expression for the decay probability that is employed in SMASH as cited in Eq. 2.18.

\subsubsection{Resonance formation in 2-to-1 reactions}

After listing the equations for the (2-body) decay, this section focuses on the reversed process. The goal is to show that when defining a 2-to- 1 cross-section $\sigma_{2 \rightarrow 1}$ (as Eq. 2.24), the general formulation of the collision probability criterion for 2-body reactions from Eq. 4.27 can be employed. For the formation the loss term reads

$$
\left|\frac{\partial f}{\partial t}\right|_{\text {coll,loss }}^{12 \rightarrow R}=\frac{1}{2 E_{1}} \int d \Gamma_{2} \int d \Gamma_{R} f_{1} f_{2} \overline{\left|\mathcal{M}_{2 \rightarrow 1}\right|^{2}}(2 \pi)^{4} \delta^{(4)}\left(P-p_{1}-p_{2}\right)
$$

where $P$ is the momentum of the formed resonance. 
The probability for the 2-to-1 resonance formation case reads

$$
P_{2 \rightarrow 1}=\frac{\Delta N_{\text {form }}}{\Delta N_{1} \Delta N_{2}}
$$

The rate for resonance formations is given as

$$
\frac{\Delta N_{\text {form }}}{\Delta t}=\Delta^{3} x \frac{\Delta^{3} p_{1}}{(2 \pi)^{3}}\left|\frac{\partial f}{\partial t}\right|_{\text {coll,loss }}^{12 \rightarrow R}
$$

With the above, the probability is found to be

$$
P_{2 \rightarrow 1}=\frac{\Delta t}{\Delta^{3} x} \frac{1}{4 E_{1} E_{2}} \int d \Gamma_{R} \overline{\left|\mathcal{M}_{2 \rightarrow 1}\right|^{2}}(2 \pi)^{4} \delta^{(4)}\left(P-p_{1}-p_{2}\right)
$$

where again the definition of $f$ as specified in Eq. 4.58 is inserted. Since the integration over $d \Gamma_{R}$ is done for a resonance, the expression for a quasiparticle has to be used as follows

$$
P_{2 \rightarrow 1}=\frac{\Delta t}{\Delta^{3} x} \frac{1}{4 E_{1} E_{2}} \int \frac{d^{4} p_{R}}{(2 \pi)^{4}} \frac{\pi}{M} \mathcal{A}_{R}(M) \overline{\left|\mathcal{M}_{2 \rightarrow 1}\right|^{2}}(2 \pi)^{4} \delta^{(4)}\left(P-p_{1}-p_{2}\right),
$$

where $\mathcal{A}_{R}(M)$ is the resonance spectral function. At this point the assumption of a constant $\overline{|\mathcal{M}|^{2}}$ has to be made in order to identify $\sigma_{2 \rightarrow 1}$ later one. The integration over $d \Gamma_{R}$ can be (trivially) performed in this case

$$
\begin{aligned}
P_{2 \rightarrow 1} & =\frac{\Delta t}{\Delta^{3} x} \frac{1}{4 E_{1} E_{2}} \overline{\left|\mathcal{M}_{2 \rightarrow 1}\right|^{2}} \int \frac{d^{4} p_{R}}{(2 \pi)^{4}} \frac{\pi}{M} \mathcal{A}_{R}(M)(2 \pi)^{4} \delta^{(4)}\left(P-p_{1}-p_{2}\right) \\
& =\frac{\Delta t}{\Delta^{3} x} \frac{1}{4 E_{1} E_{2}} \overline{\left|\mathcal{M}_{2 \rightarrow 1}\right|^{2}} \frac{\pi}{\sqrt{s}} \mathcal{A}_{R}(\sqrt{s})
\end{aligned}
$$

where the spectral function is evaluated at $\sqrt{s}=\sqrt{\left(E_{1}+E_{2}\right)^{2}-\left(\mathbf{p}_{1}+\mathbf{p}_{2}\right)^{2}}$, due to the Dirac delta functions. The matrix element for the $2 \rightarrow 1$ and $1 \rightarrow 2$ process is directly related due to the principle of detailed balance. Only the spin factors caused by the averaging over spin states have to be accounted for (more details on the matter at the beginning of the next section). The relation is

$$
\overline{\left|\mathcal{M}_{2 \rightarrow 1}\right|^{2}}=\frac{g_{R}}{g_{1} g_{2}} \overline{\left|\mathcal{M}_{1 \rightarrow 2}\right|^{2}},
$$

where $g_{k}=2 s_{k}+1$ are the spin degeneracy. $s_{k}$ is the spin of the respective state.

Due to the relation of the two matrix element, it is possible to substitute the expression in Eq. 4.64 for the decay width of the $1 \rightarrow 2$ process for the constant matrix element:

$$
P_{2 \rightarrow 1}=\frac{g_{R}}{g_{1} g_{2}} S_{12} \frac{\Delta t}{\Delta^{3} x} \frac{1}{E_{1} E_{2}} \frac{4 \pi^{2} M^{2} \Gamma_{1 \rightarrow 2}}{\lambda^{1 / 2}\left(M^{2}, m_{1}^{2}, m_{2}^{2}\right)} \mathcal{A}(\sqrt{s})
$$

Note that $\sqrt{s}=M$ still applies. According to Eq. $2.24 \sigma_{2 \rightarrow 1}$ (with adapted notation) is defined as follows

$$
\sigma_{12 \rightarrow R}=\frac{g_{R}}{g_{1} g_{2}} S_{12} \frac{2 \pi^{2}}{p_{1 \mathrm{~cm}}^{2}} \Gamma_{R \rightarrow 12} \mathcal{A}(\sqrt{s})
$$


with $p_{1 \mathrm{~cm}}=\sqrt{\left(p_{1} \cdot p_{2}\right)^{2}-m_{1}^{2} m_{2}^{2}} / \sqrt{s}=\lambda^{1 / 2}\left(M^{2}, m_{1}^{2}, m_{2}^{2}\right) /(2 \sqrt{s})$. Using the relative velocity $v_{r e l}=\lambda^{1 / 2}\left(s, m_{1}^{2}, m_{2}^{2}\right) /\left(2 E_{1} E_{2}\right)$ the probability can be rewritten in terms of $\sigma_{2 \rightarrow 1}$

$$
P_{2 \rightarrow 1}=\frac{g_{R}}{g_{1} g_{2}} \frac{\Delta t}{\Delta^{3} x} v_{r e l} S_{12} \frac{2 \pi^{2}}{p_{1 c m}^{2}} \Gamma_{R \rightarrow 12} \mathcal{A}(\sqrt{s})=\frac{\Delta t}{\Delta^{3} x} v_{r e l} \sigma_{2 \rightarrow 1}
$$

which indeed matches the general form for 2-body reactions in Eq. 4.27.

\subsubsection{Probability for multi-particle reactions}

With Eq. 4.32 the probability is defined for an assumed $n \rightarrow m$ reaction when the matrix element is known. This is, however, not the case in general. Therefore, the matrix element is assumed to be constant i.e. independent, concerning the final momenta. Thus the integration in $I_{n m}$ (Eq. 4.31) does not include integrating the matrix element anymore, but just the phase-space itself.

$$
I_{n m}=\frac{1}{S_{1^{\prime} 2^{\prime} \ldots m^{\prime}}} \int d \Phi_{m^{\prime}} \overline{\left.\mathcal{M}_{n \leftrightarrow m^{\prime}}\right|^{2}}
$$

Even though an approximation, this allows to express the probability of $n \rightarrow 2$ or $n \rightarrow 1$ in terms of the cross-section or decay-width of the reverse $2 \rightarrow n$ or $1 \rightarrow n$ reaction as already demonstrated for 2 -to- 1 reactions. This is possible since the matrix element is invariant unter time reversal.

$$
\left|\mathcal{M}_{n \rightarrow m}\right|^{2}=\left|\mathcal{M}_{m \rightarrow n}\right|^{2}
$$

But, this relation does not directly apply here since the matrix element square always appears averaged over the spin states of the initial particle and summed over the spin states of the final particles in the above equations. The averaging and summation is done as follows

$$
\overline{\left|\mathcal{M}_{n \rightarrow m}\right|^{2}}=\frac{1}{\prod_{j=1}^{n} g_{j}} \sum_{\substack{\text { initial } \\ \text { final }}}\left|\mathcal{M}_{n \rightarrow m}\right|^{2}
$$

with $g_{i}$ are the spin degeneracy factors. Therefore the more practical relation here between the forward and backward $\overline{|\mathcal{M}|^{2}}$ is given by

$$
\overline{\left|\mathcal{M}_{n \rightarrow m}\right|^{2}}=\frac{\prod_{j=1}^{m} g_{k}}{\prod_{j=1}^{n} g_{j}} \overline{\left|\mathcal{M}_{m \rightarrow n}\right|^{2}},
$$

which introduces spin factors in the equation.

Expressing the probability in terms of the cross-section or decay-width of the reverse process for different reactions classes and in turn, removing the dependency on the unknown matrix element is the focus of this section. It is also the main method to treat multiparticle reactions theoretically in this work since the matrix is unknown for the reactions of interest.

\section{Probability for 3-to-1 reactions}

As explained, the goal for the 3 -to- 1 reaction is to obtain an expression for $P_{3 \rightarrow 1}$ containing $\Gamma_{1 \rightarrow 3}$. Using Eq. 4.32 the probability for this case reads:

$$
P_{3 \rightarrow 1}=\frac{\Delta t}{\left(\Delta^{3} x\right)^{2}} \frac{1}{8 E_{1} E_{2} E_{3}} \int \frac{d^{4} p_{R}}{(2 \pi)^{4}} \frac{\pi}{M} \mathcal{A}_{R}(M) \overline{\left|\mathcal{M}_{3 \rightarrow 1}\right|^{2}}(2 \pi)^{4} \delta^{(4)}\left(P-p_{1}-p_{2}-p_{3}\right),
$$


where, since the final state particle needs to be a resonance $(R)$ for the 3 -to- 1 case, the quasi-particle phase-space integration (cf. Eq. 4.14, $M=\sqrt{s}$ ) is used.

Performing the (trivial) integration over the spectral function and assuming a constant matrix element as mentioned above leads to

$$
P_{3 \rightarrow 1}=\frac{\Delta t}{\left(\Delta^{3} x\right)^{2}} \frac{1}{8 E_{1} E_{2} E_{3}} \overline{\left|\mathcal{M}_{3 \rightarrow 1}\right|^{2}} \frac{\pi}{\sqrt{s}} \mathcal{A}_{R}(\sqrt{s})
$$

with the spectral function again being, due to the Dirac delta functions, evaluated at $\sqrt{s}=\sqrt{\left(E_{1}+E_{2}+E_{3}\right)^{2}-\left(\mathbf{p}_{1}+\mathbf{p}_{2}+\mathbf{p}_{3}\right)^{2}}$.

Considering now the decay width for the $3 \rightarrow 1$ process (as given in Eq. 4.65)

$$
\Gamma_{1 \rightarrow 3}=\frac{1}{S_{123}} \frac{1}{2 M} \overline{\left|\mathcal{M}_{1 \rightarrow 3}\right|^{2}} \Phi_{3},
$$

the matrix element in Eq. 4.83 can now be substituted (using Eq. 4.81) to obtain the final form for the 3 -to- 1 probability:

$$
P_{3 \rightarrow 1}=\frac{g_{R}}{g_{1} g_{2} g_{3}} S_{123} \frac{\Delta t}{\left(\Delta^{3} x\right)^{2}} \frac{\pi}{4 E_{1} E_{2} E_{3}} \frac{\Gamma_{1 \rightarrow 3}}{\Phi_{3}} \mathcal{A}(\sqrt{S})
$$

Note that when not using natural units $(\hbar=c=1)$, for the equation to be unit-less, its right-hand side must be multiplied by a factor of $(\hbar c)^{5}$. The 3-body phase space is given by Eq. 4.108 and $S_{123}$ is the symmetry factor for the number of identical final states of the decay process. For example, if two particles are identical in the final state, $S_{123}=2 !=2$.

Note that Eq. 4.85 also matches the equation employed in the GiBUU code $[109,301]$ for the same process, when substituting the spectral function convention in SMASH with the one from GiBUU: $\mathcal{A}_{(\mathrm{SMASH})}(M)=2 M A_{\mathrm{GiBUU}}(M)$.

\section{Generalization to $n$-to-1 reactions}

The steps from the previous section are straight-forward to generalize to any n-to- 1 reaction, which is useful in all cases where the decay width of the reverse process is known. The probability for the n-to- 1 case is given as

$$
\begin{aligned}
P_{n \rightarrow 1} & =\frac{1}{2^{n} \prod_{j=1}^{n} E_{j}} \frac{\Delta t}{\left(\Delta^{3} x\right)^{n-1}} \\
& \times \int \frac{d^{4} p_{R}}{(2 \pi)^{4}} \frac{\pi}{M} \mathcal{A}_{R}(M) \overline{\left|\mathcal{M}_{n \rightarrow 1}\right|^{2}}(2 \pi)^{4} \delta^{(4)}\left(P-\sum_{k}^{n} p_{k}\right) \\
& =\frac{1}{2^{n} \prod_{j=1}^{n} E_{j}} \frac{\Delta t}{\left(\Delta^{3} x\right)^{n-1}} \overline{\left|\mathcal{M}_{n \rightarrow 1}\right|^{2}} \frac{\pi}{\sqrt{s}} \mathcal{A}_{R}(\sqrt{s}) .
\end{aligned}
$$

For the second step the assumption of a constant matrix element is used. The decay width for this case is

$$
\Gamma_{1 \rightarrow n}=\frac{1}{S_{n}} \frac{1}{2 M} \overline{\left|\mathcal{M}_{1 \rightarrow n}\right|^{2}} \Phi_{n} .
$$

Here $S_{n}$ denotes the symmetry factor for the incoming n-body initial state of the multiparticle reaction. 
Combining the two previous equations, the general form for the probability of any decay back-reaction is obtained.

$$
P_{n \rightarrow 1}=\frac{g_{R}}{\prod_{i=1}^{n} g_{i}} S_{n} \frac{\Delta t}{\left(\Delta^{3} x\right)^{n-1}} \frac{\pi}{2^{n-1} \prod_{j=1}^{n} E_{j}} \frac{\Gamma_{1 \rightarrow n}}{\Phi_{n}} \mathcal{A}_{R}(\sqrt{s})
$$

The already discussed equations 4.77 and 4.85 are, the for this work relevant, special cases of this equation.

\section{Probability for 3-to-2 reactions}

Starting again with the general probability definition in Eq. 4.32. Note that the primed variables mark final state quantities.

$$
P_{3 \rightarrow 2}=\frac{1}{8 E_{1} E_{2} E_{3}} I_{32} \frac{\Delta t}{\left(\Delta^{3} x\right)^{2}}
$$

with the integration over the final phase space given by

$$
I_{32}=\frac{1}{S_{12}^{\prime}} \int d \Phi_{2}^{\prime} \overline{\left|\mathcal{M}_{3 \rightarrow 2}\right|^{2}}=\frac{1}{S_{12}^{\prime}} \overline{\left|\mathcal{M}_{3 \rightarrow 2}\right|^{2}} \Phi_{2}^{\prime},
$$

with $S_{12}^{\prime}$ being the now additionally needed symmetry factor for the 2-body (final) state of the reaction. For the second step the again the assumption of it being independent form the final phase space integration was made.

The cross-section for the inverse process in the center-of-mass frame is given [256] by

$$
\sigma_{2 \rightarrow 3}^{c m}=\frac{1}{S_{123}} \frac{1}{4 p_{1 c m} \sqrt{s}} \int d \Phi_{3} \overline{\left|\mathcal{M}_{2 \rightarrow 3}\right|^{2}} .
$$

With the relation $p_{1 c m} \sqrt{s}=v_{r e l} E_{1}^{\prime} E_{2}^{\prime}$ and the constant matrix element assumption, the 2-to-3 cross-section becomes

$$
\sigma_{2 \rightarrow 3}=\frac{1}{S_{123}} \frac{1}{4 v_{r e l} E_{1}^{\prime} E_{2}^{\prime}} \Phi_{3} \overline{\left|\mathcal{M}_{2 \rightarrow 3}\right|^{2}}
$$

Together Eq. 4.90 and Eq. 4.92 result in an expression independent of the unknown matrix element (by substituting it via Eq. 4.81):

$$
I_{32}=\frac{g_{1}^{\prime} g_{2}^{\prime}}{g_{1} g_{2} g_{3}} \frac{S_{123}}{S_{12}^{\prime}} \frac{\Phi_{2}^{\prime}}{\Phi_{3}} 4 v_{r e l} E_{1}^{\prime} E_{2}^{\prime} \sigma_{2 \rightarrow 3}
$$

Inserting $I_{32}$ in the probability definition, the definition for the 2-body phase space from Eq. 4.37 and $v_{r e l}$ finally results in

$$
P_{3 \rightarrow 2}=\frac{g_{1}^{\prime} g_{2}^{\prime}}{g_{1} g_{2} g_{3}} \frac{S_{123}}{S_{12}^{\prime}} \frac{1}{4 E_{1} E_{2} E_{3}} \frac{\Delta t}{\left(\Delta^{3} x\right)^{2}} \frac{\lambda}{\Phi_{3} 8 \pi s} \sigma_{2 \rightarrow 3}
$$

with $\lambda=\lambda\left(s, m_{1}^{\prime 2}, m_{2}^{\prime 2}\right)$ and $\Phi_{3}$ given by Eq. 4.108 . When not using natural units $(\hbar=$ $c=1$ ), the expression has to be multiplied by a factor of $(\hbar c)^{5}$. The expression for $P_{3 \rightarrow 2}$ only depends on the masses of the outgoing particles and the Mandelstam $s$ and therefore is valid for cases where all final particles are stable i.e. have fixed masses. The case with resonances in the final state is discussed in Section 4.1.10. Note that this probability matches the one employed in the HSD approach (given in [128] with differences in the notation). 


\section{Probability for 5-to-2 reactions}

The derivation for the 5 -to- 2 probability follows the same scheme as for 3 -to- 2 reactions. It is not presented so far in the literature. The general definition of the n-to-m probability (Eq. 4.32) for $n=5$ and $m=2$ is

$$
P_{5 \rightarrow 2}=\frac{1}{32 E_{1} E_{2} E_{3} E_{4} E_{5}} I_{52} \frac{\Delta t}{\left(\Delta^{3} x\right)^{4}}
$$

with $I_{52}=\frac{1}{S_{12}^{\prime}} \int d \Phi_{2}^{\prime} \overline{\left|\mathcal{M}_{5 \rightarrow 2}\right|^{2}}$. Assuming the matrix element independent of the final momenta, leads

$$
I_{52}=\frac{1}{S_{12}^{\prime}} \overline{\left|\mathcal{M}_{5 \rightarrow 2}\right|^{2}} \Phi_{2}^{\prime} .
$$

The cross-section for the inverse 2 -to- 5 process with this assumption is

$$
\sigma_{2 \rightarrow 5}=\frac{1}{S_{12345}} \frac{1}{4 v_{r e l} E_{1}^{\prime} E_{2}^{\prime}} \Phi_{5} \overline{\left|\mathcal{M}_{2 \rightarrow 5}\right|^{2}} .
$$

Inserting Eq. 4.97 for the matrix element in Eq. 4.96 via Eq. 4.81, the phase space integration for the final state reads

$$
I_{52}=\frac{g_{1}^{\prime} g_{2}^{\prime}}{g_{1} g_{2} g_{3} g_{4} g_{5}} \frac{S_{12345}}{S_{12}^{\prime}} \frac{\Phi_{2}^{\prime}}{\Phi_{5}} 4 v_{r e l} E_{1}^{\prime} E_{2}^{\prime} \sigma_{2 \rightarrow 5}=\frac{g_{1}^{\prime} g_{2}^{\prime}}{g_{1} g_{2} g_{3} g_{4} g_{5}} \frac{S_{12345}}{S_{12}^{\prime}} \frac{\lambda}{\Phi_{5}} \frac{1}{4 \pi s} \sigma_{2 \rightarrow 5}
$$

where $\lambda=\lambda\left(s, m_{1}^{\prime 2}, m_{2}^{\prime 2}\right)$ and the already introduced definitions for $v_{r e l}$ (Eq. 4.21) as well as $\Phi_{2}$ (Eq. 4.37) are used for the second step. Inserting this into the probability, the final formula for the 5 -to- 2 probability that depends on the cross-section for the reverse 2-to- 5 process is obtained

$$
P_{5 \rightarrow 2}=\frac{g_{1}^{\prime} g_{2}^{\prime}}{g_{1} g_{2} g_{3} g_{4} g_{5}} \frac{S_{12345}}{S_{12}^{\prime}} \frac{1}{32 E_{1} E_{2} E_{3} E_{4} E_{5}} \frac{\Delta t}{\left(\Delta^{3} x\right)^{4}} \frac{\lambda}{\Phi_{5}} \frac{1}{4 \pi s} \sigma_{2 \rightarrow 5}
$$

Note that when not using natural units $(\hbar=c=1)$, the right-hand side has to be multiplied with a factor of $(\hbar c)^{11}$ to obtain a unit-less probability. The 5-body phase space integration $\Phi_{5}$ is described in Section 4.1.7.

\section{Generalization to $n$-to-2 reactions}

Also for 2-body back-reactions, it is possible to formulate a general equation for the n-to-2 probability that can be employed in case the cross-section for the reverse 2-to-n reaction is known. Eq. 4.32 for the case of $m=2$ equals

$$
\begin{aligned}
& P_{n \rightarrow 2}=\frac{1}{2^{n} \prod_{j=1}^{n} E_{j}} \frac{\Delta t}{\left(\Delta^{3} x\right)^{n-1}} \frac{1}{S_{12}^{\prime}} \int d \Phi_{2}^{\prime} \overline{\left|\mathcal{M}_{n \rightarrow 2}\right|^{2}} \\
& =\frac{1}{2^{n} \prod_{j=1}^{n} E_{j}} \frac{\Delta t}{\left(\Delta^{3} x\right)^{n-1}} \frac{1}{S_{12}^{\prime}} \Phi_{2}^{\prime} \overline{\left|\mathcal{M}_{n \rightarrow 2}\right|^{2}} \\
& =\frac{1}{2^{n} \prod_{j=1}^{n} E_{j}} \frac{\Delta t}{\left(\Delta^{3} x\right)^{n-1}} \frac{1}{S_{12}^{\prime}} \frac{\sqrt{\lambda\left(s, m_{1}^{\prime 2}, m_{2}^{\prime 2}\right)}}{8 \pi s}\left|\mathcal{M}_{n \rightarrow 2}\right|^{2},
\end{aligned}
$$

where the matrix element is again assumed to be constant for this section and the definition of $\Phi_{2}$ (Eq. 4.37) is used in the last step. The general definition for the cross-section of a 2 -to-n process is

$$
\sigma_{2 \rightarrow n}=\frac{1}{S_{n}} \frac{1}{4 v_{r e l} E_{1}^{\prime} E_{2}^{\prime}} \Phi_{n} \overline{\left|\mathcal{M}_{2 \rightarrow n}\right|^{2}}=\frac{1}{S_{n}} \frac{1}{2 \sqrt{\lambda\left(s, m_{1}^{\prime 2}, m_{2}^{\prime 2}\right)}} \Phi_{n} \overline{\left|\mathcal{M}_{2 \rightarrow n}\right|^{2}},
$$


where Eq. 4.21 for $v_{r e l}$ is used in the second step. With the above two equations and Eq. 4.81 the probability for an arbitrary n-to-2 process is given as

$$
P_{n \rightarrow 2}=\frac{g_{1}^{\prime} g_{2}^{\prime}}{\prod_{i=1}^{n} g_{i}} \frac{S_{n}}{S_{12}^{\prime}} \frac{1}{2^{n} \prod_{j=1}^{n} E_{j}} \frac{\Delta t}{\left(\Delta^{3} x\right)^{n-1}} \frac{\lambda\left(s, m_{1}^{\prime 2}, m_{2}^{\prime 2}\right)}{\Phi_{n}} \frac{1}{4 \pi s} \sigma_{2 \rightarrow n}
$$

Equations 4.94 and 4.99 are special cases of this equation.

\section{Extension for n-to-2 reactions involving resonances}

The derived formulas for n-to-2 reactions are valid for all cases where the outgoing particles are stable i.e. have a fixed mass. All relevant n-to-2 cases for this work (Section 4.3) have stable outgoing particles. The generalization to include resonances in the final state for 2 or more particles is nevertheless noted in this section.

Reactions with resonances in the initial state can be treated with the presented equations if they are assigned a fixed mass when they scatter. This is, for example, the case in hadronic transport approaches that represent the spectral function by many (test) particles with $\delta$-like spectral functions at specific masses. The complicating factor for resonances in the final state is the integration over the final phase space that need to be done over the spectral functions. Recalling Eq. 4.1.2 one has to replace $\frac{d^{3} p}{(2 \pi)^{3} 2 E} \rightarrow \frac{d^{4} p}{(2 \pi)^{4}} \frac{\pi}{m} \mathcal{A}(m)$ in those integration. The two-body phase space then reads (assuming both particles to be resonances)

$$
\begin{aligned}
\Phi_{\tilde{2}} & =\int d \Gamma_{1} d \Gamma_{2}(2 \pi)^{4} \delta^{(4)}\left(P_{1}+P_{2}-P_{1}^{\prime}-P_{2}^{\prime}\right) \\
& =\int \frac{d^{4} p_{1}^{\prime}}{(2 \pi)^{4}} \frac{d^{4} p^{\prime}}{(2 \pi)^{4}} \pi \frac{\mathcal{A}_{1}\left(m_{1}\right)}{m_{1}} \pi \frac{\mathcal{A}_{2}\left(m_{2}\right)}{m_{2}}(2 \pi)^{4} \delta^{(4)}\left(P_{1}+P_{2}-P_{1}^{\prime}-P_{2}^{\prime}\right) \\
& =\int \frac{d^{3} p_{1}^{\prime}}{(2 \pi)^{3}} \int d p_{1}^{\prime 0} \frac{\mathcal{A}_{1}\left(m_{1}\right)}{2 m_{1}} \\
& \times \int \frac{d^{3} p_{2}^{\prime}}{(2 \pi)^{3}} \int d p_{2}^{\prime 0} \frac{\mathcal{A}_{2}\left(m_{2}\right)}{2 m_{2}}(2 \pi)^{4} \delta^{(4)}\left(P_{1}+P_{2}-P_{1}^{\prime}-P_{2}^{\prime}\right)
\end{aligned}
$$

with $d p^{0}=d m \frac{m}{\sqrt{\left(m^{2}+p^{2}\right)}}$ following from $m^{2}=p_{0}^{2}-p^{2}$. The $\tilde{2}$ notation signifies a resonance in the final state here. If not all particles are resonances, the respective $d \Gamma$ integration, of course, still simplifies to $\frac{d^{3} p}{(2 \pi)^{3} 2 E}$. The formula in Eq. 4.37 does not apply anymore since it includes additional integrations over the spectral function, which need to be calculated for the given resonance (spectral function). Therefore it also cannot be used to simplify $\Phi_{2}$ in the probability definition as done for the 3-to- 2 and 5-to- 2 case above. The discussion in this section is meant as a starting point to derive the full equations for the case of resonances in the final state to be studied in the future.

Note that for the reverse 2-to-n case, the probability as defined in Eq. 4.27 is valid even for the case of resonances in the final state, since the complexity of the additional integrations over the spectral function is included in the $\sigma_{2 \rightarrow n}$ cross-section. 


\section{Summary}

This concludes the comprehensive presentation of the necessary theoretical background of the stochastic rates approach. The collision probabilities are derived from the Boltzmann equation. The probability is defined as the fraction of a number of collisions over all possible particle pairings in a given phase-space cell. The main assumption of the derivations is to consider the matrix element to be independent of the final state momenta (i.e. constant). For this case, the collision probabilities can be expressed in terms of the known decay width or cross-section of the reverse process. The main results are collision probabilities derived for binary, 3-body and 5-body collisions, which are displayed in Eq. 4.27, Eq. 4.85, Eq. 4.94 and Eq. 4.99. Furthermore, resonance formation as a special case of binary collisions (Section 4.1.9), the decay treatment (Section 4.1.8) and the generalization to arbitrary $n \rightarrow\{1,2\}$ reactions (Section 4.1.10) are discussed. The generalizations of the approach with a constant matrix element and its application to 5body collisions are new contributions of this thesis. Overall, the theoretical prerequisite to realize a stochastic collision criterion and multi-particle reactions in a transport approach are thereby described. 


\subsection{Stochastic collision criterion in transport approaches}

The realization of a stochastic collision criterion for binary scattering on the example of the hadronic approach SMASH is discussed in this section. The criterion of whether particles interact in a transport approach is based on the above-derived collision probabilities. They are used for a random decision of whether particles scatter. A stochastic collision criterion is already applied in serval other transport approaches, where it is often referred to as the local-ensemble method $[134,126,128,111,109,129,130]$ (see Section 1.2.6 and the introduction of this chapter for more details). A stochastic treatment employed for binary interactions is in principle ${ }^{3}$ equivalent to a geometric collision criterion (as described in Section 2.2.2). The extension to include multi-particle reactions is discussed in the following section. The final probability equations that are employed in SMASH are repeated in the following.

The employed collision probability for binary reactions is defined in terms of the crosssection as

$$
P_{2 \rightarrow m}=\frac{\Delta t}{\Delta^{3} x} v_{\mathrm{rel}} \sigma_{2 \rightarrow m},
$$

with the relative velocity $v_{\text {rel }}$ given by Eq. 4.21. This equation is derived in Section 4.1.4 and a special case $(n=2)$ of the general equation for the $n \rightarrow m$ collision probability given in Eq. 4.32. The probability is employed for all two-body scatterings, including string processes $(m \leq 2)$ and resonance formation. For the latter, it is shown in Section 4.1.9 that, for the case of resonance formation $(n=2, m=1)$ the cross-section coincides with $\sigma_{2 \rightarrow 1}$ used in SMASH as given in Eq. 2.24. Note that the probability for a decay process $(n=1)$ is defined as

$$
P_{1 \rightarrow m}=\Delta t \frac{M}{E_{1}} \Gamma_{1 \rightarrow m},
$$

where $m$ is the number of final particles. As discussed in Section 4.1.8, this decay probability is the same already used in SMASH (Eq. 2.18), therefore the decay treatment does not have to be altered for the stochastic criterion. As explained in Section 4.1.6, when employing test particles, the probability needs to be scaled:

$$
P_{n \rightarrow m}^{\prime}=\frac{P_{n \rightarrow m}}{N_{\text {test }}^{n-1}} .
$$

The numerical realization of the stochastic criterion is the same for all (stochastic) scatterings, including the new multi-particle reactions. The space is divided into equally sized cells $\left(\Delta^{3} x\right)$. The probability is calculated for all n-particle combinations within each timestep, so only particles within a cell can interact. $n$ is chosen according to the number of particles that can interact (so $n=2$ is the default for binary reactions and if multiparticle reactions are used, then $n>2)$. The calculated probability $(0 \leq P \leq 1)$ is used for a Monte-Carlo decision i.e. if a generated random number between 0 and 1 is smaller or equal to the probability, the reaction is accepted. The collision time is randomly chosen within the given timestep $\Delta t$. In case multiple reactions for the same particle are accepted, only the first reaction is performed. Newly created particles in inelastic collisions are placed at the position of a random incoming particle to avoid density artifacts. In contrast to the geometric criterion, the stochastic criterion is a strictly timestep-based method. The timesteps, therefore, have to be chosen small enough that the assumption

\footnotetext{
${ }^{3}$ Both criteria are applicable within certain limits related e.g. to boost invariance or calculation parameters that might lead to differences. See Section 5.1 for examples.
} 
that each particle only interacts once per timestep is justified and the defined probability is not exceeding 1 for a given $\Delta^{3} x$. Also, the cells $\Delta^{3} x$ have to be chosen sufficiently small, since only in the limit of $\Delta^{3} x \rightarrow 0$ (and $\Delta t \rightarrow 0$ ) the numerical solution matches the exact solution of the Boltzmann equation. At the same time, the cells still have to be sufficiently filled with particles. Choosing an appropriate pair of $\Delta^{3} x$ and $\Delta t$ and the sensitivity of the results to this choice is discussed in detail in Section 5.1.5. The stochastic collision criterion is introduced as an option with the version SMASH-1.7 [212]. Further details of the implementation and numerical realization are given in Appendix A.

\subsection{Multi-particle reactions in SMASH}

The difficulty of describing multi-particle reactions generally arises from the collision criterion. In transport approaches, the most common criterion whether particles collide is the geometric criterion (Section 2.2.2). So far, no generalization of this criterion to multiparticle reactions is available. The stochastic collision criterion, on the other hand, is easily generalized to multi-particle reactions.

The main purpose of introducing the stochastic criterion in SMASH is to treat multiparticle reactions while adhering to detailed balance. It is applied to individual reactions that are known to be relevant and interesting (Section 1.3): the mesonic Dalitz decay back-reaction (3-to-1), the deuteron catalysis reactions (3-to-2) and the proton-antiproton annihilation back-reaction (5-to-2). Note that detailed balance is not globally conserved for all calculations due to string fragmentation, which emits multiple particles. back-reactions are not introduced for such reactions in this work.

With a collision criterion that only is able to perform binary collisions (like the geometric one in SMASH), an alternative approach to model multi-particle reaction is to employ a chain of reaction of binary collisions (e.g. $\pi \pi \pi \rightarrow \rho \pi \rightarrow \omega$ for $\pi \pi \pi \rightarrow \omega$ ). Detailed balance is conserved for every step and therefore also for the overall reaction. This multi-step construct with intermediate resonances is employed in SMASH in several places to adhere to detailed balance with a geometric criterion. The direct, stochastic multi-particle reactions allow to constrain the effect that this helper construct has on the results, which is done throughout the following results. Note that such a reaction chain approach is only wellmotivated in instances where fitting intermediate reactions are known. On the contrary, the stochastic treatment allows treating multi-particle reactions directly for arbitrary reactions. Additionally, it is more rigoros since it is directly derived from the collision integral of the Boltzmann equation and therefore more closely follows kinetic theory expectations (Section 5.1.2).

In the following sections, the different multi-particle reactions in SMASH are introduced ${ }^{4}$. They are based on the derived probability equations in Section 4.1. The matrix element is assumed to be independent of the final momenta here to express the probabilities in terms of decay width or cross-section of the reverse process and avoid needing to employ a generally unknown matrix element. More details on implementation and practical information for SMASH calculations with multi-particle reactions are given in Appendix A. In Appendix A.4, also a discussion of the increased computing time that is required to account for the larger combinatorics of multi-particle reactions is included.

\footnotetext{
${ }^{4}$ Three-body reactions are available since SMASH-2.0 [213] and the 5-to-2 action will be part of the next tagged version of SMASH (probably SMASH-2.1).
} 


\subsubsection{Meson 3-to-1 reactions}

The $3 \rightarrow 1$ probability is applied in SMASH for a set of known three-body decay (back-) reactions of mesonic resonances: $\pi \pi \pi \rightarrow \omega, \pi \pi \pi \rightarrow \phi$ and $\pi \pi \eta \rightarrow \eta^{\prime}$. In particular, for the $\omega$ and $\eta^{\prime}$, the three-body decay is the dominant decay channel. With the stochastic criterion, it is now possible to treat the multi-particle back-reaction directly, while fulfilling detailed balance. The back-reaction had to be treated in two steps with a resonance in the intermediate state with the geometric criterion. For the $\omega$ the reaction chain reads $\pi \pi \pi \rightarrow \rho \pi \rightarrow \omega$. The same chain is also used for the $\phi$. In the case of the $\eta^{\prime}$, the $\sigma$ is needed as an intermediate step; $\pi \pi \eta \rightarrow \sigma \eta \rightarrow \eta^{\prime}$. Note that the $\omega$ is a special case, since it might actually decay (in part) through $\omega \rightarrow \rho \pi$ [247]. Whereas, in the other cases, including the multi-step treatments of 3-to- 2 and 5 -to-2, are mainly motivated by modeling a multi-particle reaction with detailed balance in a binary collision approach.

The formula for the probability is

$$
P_{3 \rightarrow 1}=\frac{g_{R}}{g_{1} g_{2} g_{3}} S_{123} \frac{\Delta t}{\left(\Delta^{3} x\right)^{2}} \frac{\pi}{4 E_{1} E_{2} E_{3}} \frac{\Gamma_{1 \rightarrow 3}}{\Phi_{3}} \mathcal{A}(\sqrt{S})
$$

as derived in Section 4.1.10 (same as Eq. 4.85) with assumption of matrix element that is independent of the final momenta. The 3-body phase space is defined following the convention in the PDG Book [256]

$$
\Phi_{3}=\frac{1}{(2 \pi)^{3}} \frac{1}{16 M^{2}} I_{3},
$$

with the integral $I_{3}$ defined as

$$
I_{3} \equiv \int d m_{12}^{2} d m_{23}^{2}=\int_{\left(m_{1}+m_{2}\right)^{2}}^{\left(M-m_{3}\right)^{2}}\left[\left(m_{23, \max }^{2}-m_{23, \min }^{2}\right)\right] d m_{12}^{2} .
$$

The min and $\max$ values are defined as in the PDG book (Eq. 4.42). See Section 4.1.7 for more possible definitions and details of $\Phi_{3}$. The 3-body phase space is integrated explicitly for all possible reaction pairs. For an accepted reaction, the outgoing particles are sampled isotropically. The treatment of the $1 \rightarrow 3$ is the same as for all other decays in SMASH, where for three-body decays the width is not mass-dependent but assumed to be constant.

The needed symmetry and degeneracy factors for $\pi^{+} \pi^{-} \pi^{0} \rightarrow \omega, \pi^{+} \pi^{-} \pi^{0} \rightarrow \phi, \pi^{+} \pi^{-} \eta \rightarrow$ $\eta^{\prime}$ and $\pi^{+} \pi^{-} \eta \rightarrow \eta^{\prime}$ reactions are $g_{\omega}=g_{\phi}=3, g_{\pi}=g_{\eta}=g_{\eta^{\prime}}=q, S_{\pi^{+} \pi^{-} \pi^{0}}=S_{\pi^{+} \pi^{-} \eta}=1$ ! and $S_{\pi^{0} \pi^{0} \eta}=2$ !.

\subsubsection{Deuteron 3-to-2 reactions}

The next class of multi-particle reactions realized with the stochastic collision criterion are $3 \leftrightarrow 2$ reactions. These are needed for the microscopic description of creation and destruction of deuterons in the hadron gas. The expression for $P_{3 \rightarrow 2}$ is derived in Section 4.1.10 with the assumption of the matrix element being independent of the final momenta. The probability for the 2-to-3 process is given by Eq. 4.104 as for all binary scatterings.

The $P_{3 \rightarrow 2}$ probability is applied to the deuteron formation catalysis reactions $\pi p n \leftrightarrow \pi d$, $N p n \leftrightarrow N d$ and $\bar{N} p n \leftrightarrow \bar{N} d$. The probability for a 3 -to-2 process is given as

$$
P_{3 \rightarrow 2}=\frac{g_{1}^{\prime} g_{2}^{\prime}}{g_{1} g_{2} g_{3}} \frac{S_{123}}{S_{12}^{\prime}} \frac{1}{4 E_{1} E_{2} E_{3}} \frac{\Delta t}{\left(\Delta^{3} x\right)^{2}} \frac{\lambda}{\Phi_{3} 8 \pi s} \sigma_{2 \rightarrow 3}
$$


(same as Eq. 4.94). The 3-body phase space $\left(\Phi_{3}\right)$ treatment is the same as for 3-to-1 reactions. $\sigma_{2 \rightarrow 3}$ (i.e. $\sigma_{\pi d}$ and $\sigma_{\pi N}$ ) is fitted to available experimental data in [183]. The symmetry factors for the reactions $\pi p n \rightarrow \pi d, \bar{N} p n \rightarrow \bar{N} d$ and their charge conjugated are $\frac{S_{\pi p n}}{S_{\pi d}^{\prime}}=1$, while for $N p n \rightarrow N d$ and its charge conjugate, as there are 2 identical particles in the initial state, $\frac{S_{N p n}}{S_{N d}^{\prime}}=2$. The spin degeneracies read $g_{d} / 3=g_{N, p, n} / 2=g_{\pi}=1$.

Previous to this work, the deuteron catalysis reaction had to be treated via a binary reaction chain involving a fake resonance. The $3 \leftrightarrow 2$ reaction is broken down into two steps with a fake decaying resonance (called $d^{\prime}$ with the decay mode $d^{\prime} \leftrightarrow n p$ ) in the intermediate step, so that the reaction (chain) only contains 2-body collisions: $X d \leftrightarrow X d^{\prime} \leftrightarrow X n p$. The properties of the $d^{\prime}$ are chosen to reproduce the $\pi d, N d$ and $\bar{N} d$ cross-sections and to achieve a lifetime that lasts the time $n$ and $p$ spend flying by each other. This treatment of the deuteron reactions is introduced in detail in [183] and is labeled in the results as the 2-to-2 stochastic or 2-to-2 geometric treatment depending on the employed collision criterion. The direct 3-to-2 treatment is labeled as 3-to-2 stochastic. All treatments are employed and compared for the results (Section 5.3).

\subsubsection{Proton-antiproton 5-to-2 reactions}

The third multi-particle treatment in SMASH concerns $5 \leftrightarrow 2$ reactions. The specific reaction of interest is $5 \pi \rightarrow N \bar{N}$, which is the detailed balance restoring back-reaction of $N \bar{N}$ annihilation. While the $5 \pi$ reaction is desired equivalently for $p$ and $n$, the $5 \pi \rightarrow \mathrm{p} \overline{\mathrm{p}}$ reaction is the main interest since protons are experimentally more accessible. The results (Section 5.4) therefore focus on discussing the proton reaction.

The collision probability for a 5 -to- 2 reaction under the assumption that the matrix element only depends on the initial center-of-mass energy is

$$
P_{5 \rightarrow 2}=\frac{g_{1}^{\prime} g_{2}^{\prime}}{g_{1} g_{2} g_{3} g_{4} g_{5}} \frac{S_{12345}}{S_{12}^{\prime}} \frac{1}{32 E_{1} E_{2} E_{3} E_{4} E_{5}} \frac{\Delta t}{\left(\Delta^{3} x\right)^{4}} \frac{\lambda}{\Phi_{5}} \frac{1}{4 \pi s} \sigma_{2 \rightarrow 5}
$$

as derived in Section 4.1.10 (same as Eq. 4.99). For the annihilation reaction, $S_{\mathrm{p} \overline{\mathrm{p}}}=1$ and $S_{\pi^{+} \pi^{+} \pi^{-} \pi^{-} \pi^{0}}=2 ! 2 !=4$ as well as $g_{p / \bar{p}}=2$ and $g_{\pi}=1$. As this collision probability is calculated for all possible 5- (and 2-) particle combinations, calculations including 5-to-2 reactions are computationally expensive due to involved combinatorics.

The 5-body phase space is parametrized, when it is employed for the collision probability above, which saves part of the computational expenses. The general form of the parametrization chosen to fit the numerically sampled 5-body phase space (according to Eq. 4.52) for the case of 5 pions $\left(m_{i}=m_{\pi}\right)$ is

$$
f_{n}(s)=A\left(s-s_{0}\right)^{5}\left(1+\left(\frac{s}{s_{0}}\right)^{n}\right)^{\frac{-\alpha}{n}}
$$

where $s_{0}=25 m_{\pi}^{2}$. The order $\mathrm{n}=1$ already fits the phase space sufficiently, so the final form for parametrization is

$$
f_{1}(s)=A\left(s-s_{0}\right)^{5}\left(1+\frac{s}{s_{0}}\right)^{-\alpha}
$$

with the parameter values $A=2.1018 \cdot 10^{-10}$ and $\alpha=1.92802$. Figure 4.3 shows the comparison of the numeric result with the parameterization and the analytic form from Eq. 4.53. The plot verifies first that the numeric sampling of Eq. 4.52 is correct, since for 


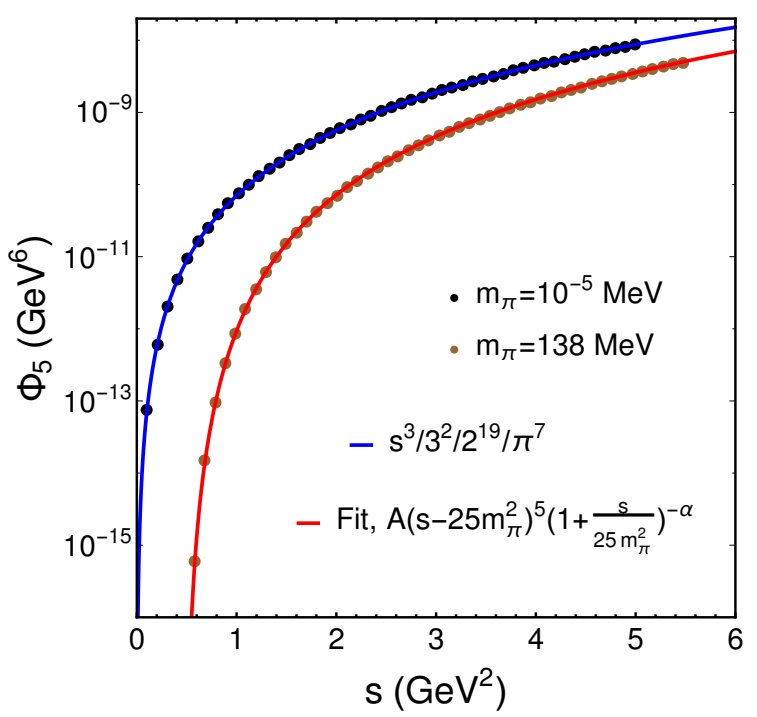

Figure 4.3: Numeric result for $\Phi_{5}$ in the case of five pions $\left(m_{i}=m_{\pi}\right)$ compared with the parameterization from Eq. 4.113 and the numeric result for the almost massless case $\left(m_{i}=10^{-5} \mathrm{MeV}\right)$ compared with analytic expression for the massless case (Eq. 4.53) [302].

the almost massless case of $m_{i}=10^{-5} \mathrm{MeV}$, it matches the analytic form. Furthermore, the parametrization also shows an excellent agreement for $m_{i}=m_{\pi}$, verifying the fitting procedure. Note that for the 2 -to-5 process, the 5 -body final state is (also) sampled isotropically (details are found in Appendix A).

An alternative treatment to fulfill detailed balance for $\mathrm{N} \bar{N}$ annihilations is a chain of two-body reactions via intermediate resonances (referred to as the resonance treatment in the following), which is possible to employ with the geometric criterion. As such, the annihilation of $\mathrm{N}$ and $\overline{\mathrm{N}}$ is performed via $\mathrm{N} \overline{\mathrm{N}} \rightarrow \mathrm{h}_{1} \rho$, which subsequently decays to $5 \pi$ (via $\mathrm{N} \overline{\mathrm{N}} \rightarrow \mathrm{h}_{1} \rho \rightarrow \rho \pi \pi \pi \rightarrow 5 \pi$ ). This multi-step process, in turn, provides the backreaction $5 \pi \rightarrow \mathrm{N} \overline{\mathrm{N}}$, needed for the restoration of $\mathrm{NN}$ pairs. Detailed balance is conserved for all intermediate reactions and therefore for the 5-to-2 reaction overall. Note that the $\mathrm{N} \overline{\mathrm{N}} \rightarrow \mathrm{h}_{1} \rho$ is experimentally not constrained and this reaction chain construct only allows to treat a 5-body final state. Also, the reaction is slowed down by introducing the intermediate resonances, which exist a certain time before their decay. The effect of this on the final result will be gauged by the comparison to the direct stochastic treatment. When proton-antiproton annihilations are realized via string fragmentation, the back-reaction is unaccounted for and detailed balance is broken.

The treatment of $N \bar{N}$ annihilation in this work makes two approximations:

First, the cross-section of the annihilation reaction is defined as the remainder of the parametrized total $\bar{p} \bar{p}$ cross-section after subtracting its elastic contribution. This approximation assumes that the inelastic cross-section is saturated with annihilation reactions and neglects the remaining sub-leading inelastic contributions [303, 304, 128] (while still matching the total cross-section). The cross-section for the annihilation reaction for both

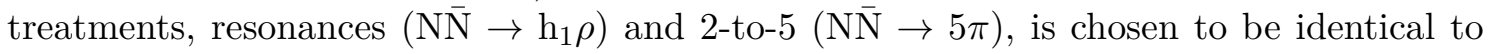
ensure comparability. The $n \bar{n}$ cross-section is treated analogously. As depicted in Figure 4.4, defining the annihilation cross-section as the full inelastic (i.e. total minus elastic) cross-section naturally lead to an agreement of the total cross-sections of resonance and 2-to-5 treatment with the total parametrization. In the meantime, this overestimates the 


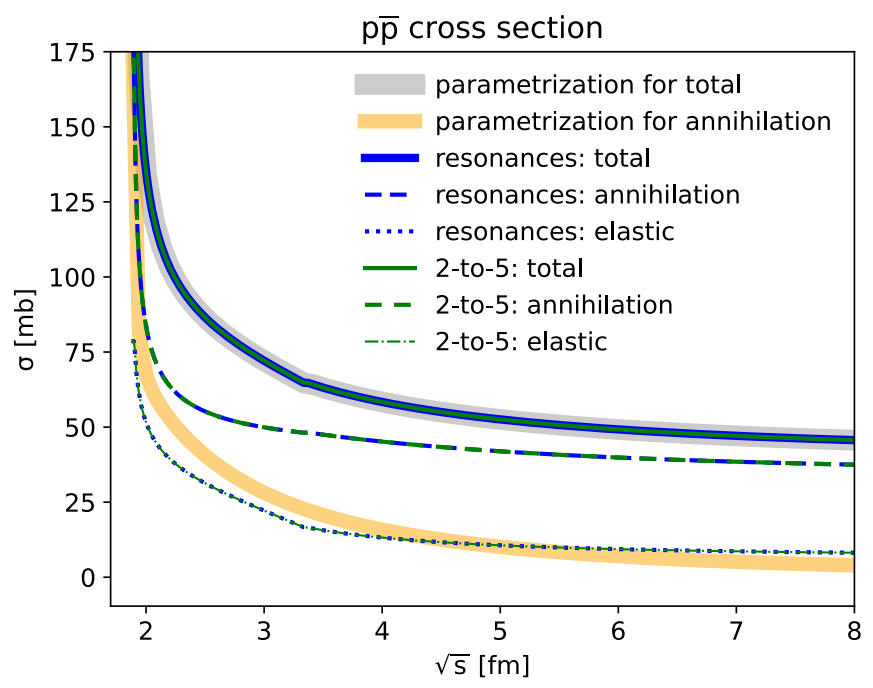

Figure 4.4: $p \bar{p}$ cross-sections a function $\sqrt{s_{N N}}$.

parametrization of the annihilation cross-section data as a compromise. The parameterizations are tuned to match the available experimental data and are taken from [106].

Second, only the most likely $N \bar{N}$ annihilation final state is considered. In principle, the whole set of processes $N \bar{N} \rightarrow m \pi$, with $m=2,3 \ldots$ pions [304] is possible. However, the most likely reaction is $m=5$ [304] for the typical energies of the $\mathrm{N}(\bar{N})$ in the employed afterburner calculation ( $\sqrt{s}$ of $N \bar{N}$ is close to the two-nucleon mass threshold). Therefore, the set of annihilation processes is effectively modeled by $N \bar{N} \rightarrow 5 \pi$ as an average. 



\section{Chapter 5}

\section{Results with multi-particle collisions}

The primary goal of this thesis is to study a gas of hadrons, as it is produced in heavyion collisions when including the reaction of multiple particles. The employed reactions are the back-reaction of mesonic Dalitz decays (3-to-1), the deuteron catalysis reaction (3-to-2) and the 5-pion back-reaction of nucleon-antinucleon annihilations (5-to-2). They are realized in the hadronic transport approach SMASH, as introduced in the previous chapter together with the underlying theory and the stochastic collision criterion.

The main results for realistic heavy-ion collision scenarios are found in the last three sections. They include an investigation of the effects of 3-to- 2 and 5-to- 2 reactions on the deuteron and proton abundances in the dilute non-equilibrium stages of a heavy-ion collision (Section 5.3 and Section 5.4). Thereby, the results address topical research questions (Section 1.3) about the general relevance of non-equilibrium rescatterings, the "snowballs in hell"-paradox i.e. the time of $d$ formation and the significance of $\bar{p} \bar{p}$ regeneration by $5 \pi$. Furthermore, the relevance of mesonic Dalitz decays over the whole collision evolution is explored (Section 5.2).

Before these topical questions are addressed, the extensions to the hadronic transport approach are carefully and extensively verified (Section 5.1). For this, the new stochastic collision criterion is mainly employed in infinite matter calculations to test the equilibrium properties of the different stochastic reactions. The stochastic criterion is theoretically only a complete solution of the Boltzmann equation for infinite and infinitesimal values of timestep, cell size and test particle number. Since this requirement is numerically only approximated, also the numerical stability when varying these calculation parameters is thoroughly explored. 


\subsection{Stochastic rates in and out-of equilibrium}

The stochastic rates extension to SMASH, introduced in Chapter 4, is verified in this section by demonstrating an agreement of the stochastic rates with analytic results or the geometric criterion. The stochastic rates are tested for simplified systems, where analytic expectations for the scattering rate, the equilibrated yield or the equilibration process are available. First, equilibrium systems in a box are studied with only elastic (Section 5.1.1) or a limited set of inelastic reactions (Section 5.1.2). A particular focus is placed on the observation of detailed balance. In addition, the equilibration process for direct multi-particle reactions is compared with binary reaction chains that model the same multi-particle reaction effectively. Then, more realistic hadron gases are studied in a sphere or collider scenario, which include out-of-equilibrium phases (Section 5.1.3 and Section 5.1.4). The scattering rate and multiplicity of stochastic and geometric criterion are compared to validate their equivalence for binary scatterings in a realistic medium. Also, the numerical stability for different calculation parameters is verified (Section 5.1.5). Albeit a rather numerical-technical aspect, understanding the dependence of the results on calculation parameters is an important prerequisite before addressing more exciting physical questions. A brief summary of the most important findings is given at the end of the section.

Even though only few physically relevant results are presented in this section, the presented detailed verification helps to establish the new framework for stochastic rates. This is one of the main achievements of this work since it provides not only the basis for the later presented studies, but also ample opportunities for future multi-particle studies.

\subsubsection{Elastic scattering rate}

To test if the collision number adheres to the kinematic theory prediction $\left(\mathrm{N}_{\text {coll }}^{\text {theory }}\right)$, a basic box scenario (as explained in Section 2.3) is chosen. The stochastic criterion has to match the theoretical value since both are directly based on the same theory. The box is filled with neutral pions that only scatter elastically with a fixed elastic cross-section. To verify the stochastic criterion for 2-to-2 elastic reactions, six different calculation parameters are varied while keeping the others fixed. The different parameters are noted in the figures. Shown is always the ratio of the number of collisions observed over the theoretical prediction.

The results are seen in Figure 5.1. No dependency from the test particle number or temperature is observed. While this is the ideal case, the other results are also important since they outline the limits of the new stochastic collision approach. Deviations in the form of a too low scattering rate are observed for large timestep sizes, for small box volumes and large particle numbers (at small $N$ there is no deviation since the error bars are compatible with 1). Also, towards large elastic cross-sections, the scattering rate slightly drops. The deviations that show are not caused by the stochastic criterion per se, but by strictly using timesteps for the system propagation (see also Section 5.1). This implies the assumption that only one collision per timestep per particle happens, which breaks down if the timesteps become too large. Whether a timestep is too large depends on the density since the denser the system is, the more collisions should happen. This is nicely seen for the different plots in Figure 5.1. If the density is increased (by varying $N$ or $V$ ) the timestep becomes too large, leading to the observed deviations in the results. Along the same line, if the cross-section is increased, too many (more than one) collisions are supposed to happen inside the timestep; therefore, the scattering rate drops. It was 


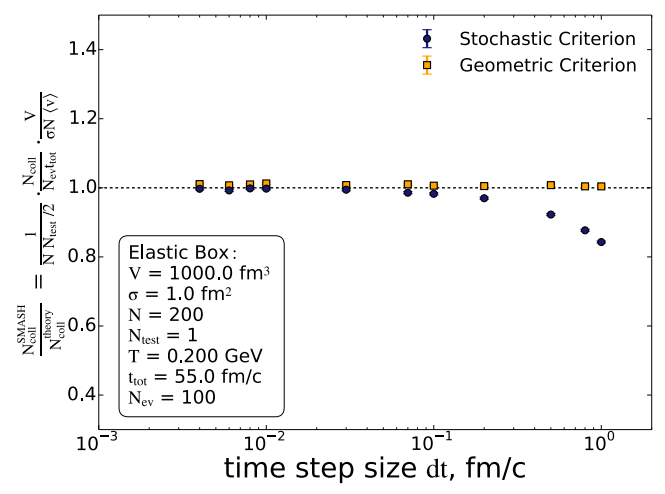

(a)

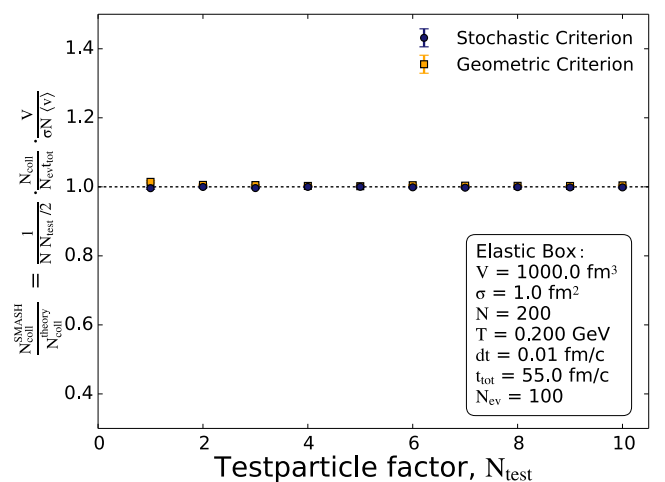

(c)

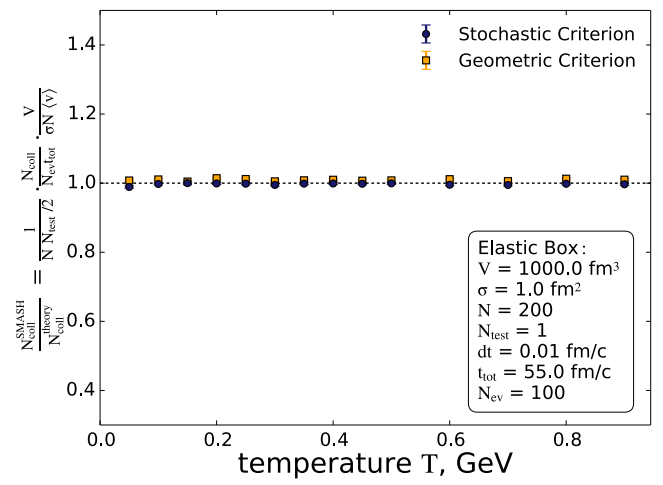

(e)

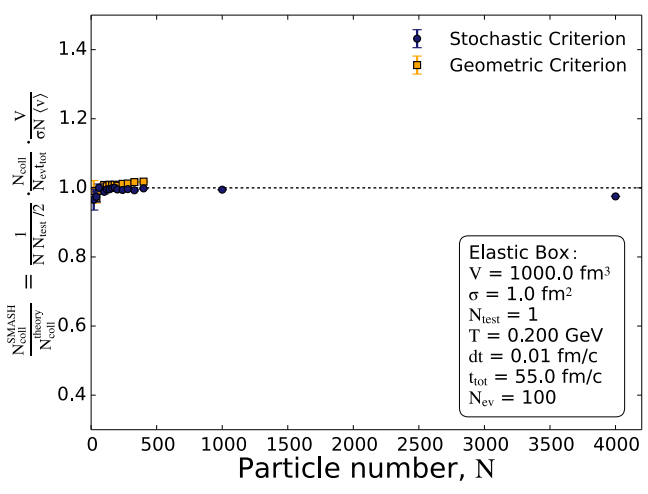

(b)

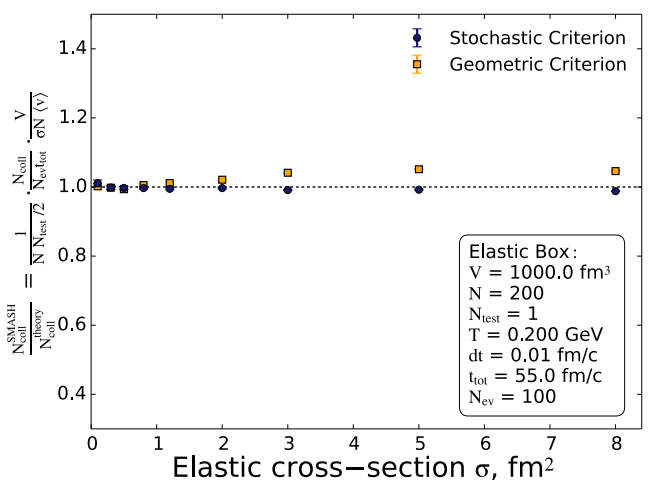

(d)

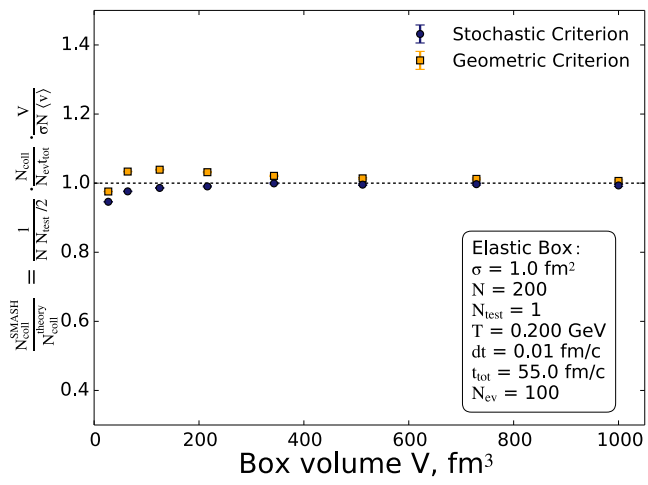

(f)

Figure 5.1: Ratio of the calculated over the theoretical expected scattering rate in an elastic box. 
checked that with a smaller timestep size of $d t=0.001$, the cross-section plots also meet the analytical expectation for the largest cross-section values.

Overall, it is seen that the stochastic criterion, apart from the discussed deviations in extreme parameter regions, exactly matches the theoretical expectation for the two-body elastic scattering rate. The agreement is generally better than for the geometric criterion.

\subsubsection{Equilibration and detailed balance of the stochastic criterion}

Conserving detailed balance for reactions with more than two outgoing particles and therefore being able to employ processes with more than 2 particles one of the main motivations of employing the stochastic criterion. In the following, it is verified that detailed balance is conserved for the newly introduced multi-particle reactions. These results are the first results for multi-particle reactions in the transport approach SMASH. As the test system, different homogenous, infinite matter (i.e. box) scenarios (as explained in Section 2.3) are chosen that only include a simplified set of relevant reactions and degrees of freedom. The results extend the test of the scattering rate for elastic collisions in the previous section by probing (different) inelastic reactions and the equilibration properties of the system in more detail. These systematic tests form the basis for studying dynamic non-equilibrium systems like nucleus-nucleus collisions that are measured in experiment.

For simple systems, it is possible to test detailed balance and straightforward to calculate analytic expectations for the equilibrated multiplicities and the equilibration process. Comparisons to these analytic expectations allow to further test and validate the approach for stochastic rates. This includes the verification of the derived and implemented formulas for the probability. Any errors in the symmetry and degeneracy factors would be immediately apparent by a disagreement with the analytic results. The discussed (thermal) analytic expectations have to be matched since the stochastic rates are rigorously based on the Boltzmann equation (as shown in Section 4.1).

This section is structured as follows: After some general remarks on how the transport and analytical results are obtained, results for simple mesonic boxes, testing the stochastic (and geometric) criterion for inelastic 2-body reactions, are shown. Afterward, the equilibration and detailed balance for all in this work newly-introduced multi-particle reactions, $3 \leftrightarrow 1$, $3 \leftrightarrow 2$ and $5 \leftrightarrow 2$, is verified.

\section{Analytic calculations}

A few theoretical and technical remarks are necessary to explain before presenting the box calculation results.

To compare with the thermal multiplicity expectation for the ideal gas, it is necessary to extract the temperate of the equilibrated system, which can differ from the initial temperature due to the inelastic reactions between particles. The temperature is measured by fitting the energy spectrum of the particles inside the box, which equilibrium form is assumed to be thermal i.e. proportional to $T$ as follows

$$
\frac{1}{E \sqrt{E^{2}-m^{2}}} \frac{d N}{d E} \propto e^{\frac{-E+\mu}{T}} .
$$

Since $\pi$ are an abundant species for all systems, the energy spectrum of one $\pi$ species $\left(\pi^{+}\right)$ is fitted to ensure the most accurate temperature. 
The thermal particle density $n$ for the extracted $T$ and particle with mass $m$ is given by the grand-canonical ideal gas expectation

$$
n=\frac{g m^{2} T}{2 \pi^{2}(\hbar c)^{3}} e^{\left(\frac{\mu_{i}}{T}\right)} K_{2}\left(\frac{m}{T}\right)
$$

where $g$ is the spin degeneracy, $\mu_{i}$ the relevant chemical potentials like baryon and strangeness chemical potential (normally 0 for the following results) and second-order Bessel function $K_{2}$. The thermal particle multiplicity $N^{t h}$ i.e. number of particles in the box is obtained by multiplying with the box volume: $N^{t h}=n V$. Unfortunately, the thermal multiplicity cannot be compared directly with the following results, since the degrees of freedom and interactions are simplified. A comparison would only be valid for the full hadron gas, where the densities are independent from each other. In the case of only employing specific particles and reactions, the chemical potential $\mu$ would need be calculated specifically, since the expected multiplicity is modified: $N_{i}=N_{i}^{t h} e^{\mu_{i} / T}$.

It is however possible to compare with a ratio of $N^{t h}$ in order to avoid the extra step of computing the specific chemical potential. Assume as an example a box with just one $2 \leftrightarrow 1$ reaction $(A \leftrightarrow B B)$. In this case $2 N_{A}+N_{B}=$ const., and therefore the chemical potentials are assigned as $\mu_{A}=2 \mu$ and $\mu_{B}=\mu$. The chemical potential then could be obtained by solving the equation $2 N_{A}^{\text {init }}+N_{B}^{\text {init }}=$ const. $=N_{A}^{t h} e^{2 \mu / T}+N_{B}^{t h} e^{\mu / T}\left(N_{i}^{\text {init }}\right.$ are the multiplicity values at initialization). But it is also possible to take the ratio $R=\frac{N_{A}}{N_{B}^{2}}$, where the chemical potential cancels and the observed ratio equals the ratio of thermal multiplicities:

$$
R=\frac{N_{A}}{N_{B}^{2}}=\frac{N_{A}^{t h} e^{2 \mu / T}}{N_{B}^{t h} e^{2 \mu / T}}=\frac{N_{A}^{t h}}{N_{B}^{t h}}
$$

The ratio of the equilibrated number of particles inside the boxes are therefore directly comparable to the ratios calculated with Equation 5.2. The equilibrated multiplicity thereby are verified analytical. Since the stochastic criterion is directly rooted in kinetic theory, an approach employing the stochastic criterion needs to agree with these analytic values.

The second analytic comparison performed in this section is based on so called rate equations. While more involved to calculate, the analytic result are time-dependent multiplicities, from initialization to their equilibrium values. The comparison to those provide an even more thorough examination of the presented stochastic rate approach. The needed equations are also based on thermodynamics with the idea introduce in [194]. It is essentially an extension of the arguments made above for the multiplicity ratios. Solving the couple system of rate equations results in the yield over time relaxing to its equilibrium values. A difference to [194] here is that the considered systems are not expanding, but static boxes. For this, the system is assumed to be in kinetic equilibrium with a constant temperature. The equilibrium yield is again described by a grand-canonical ensemble. But since the particle species are not assumed to be chemically equilibrated, every species is assigned a fugacity $\lambda_{i}$, which time dependence needs to be solved for in the end. The time dependent particle multiplicity is therefore defined as

$$
\begin{aligned}
N_{i} & =V n_{i}^{t h}(T) \lambda_{i}, \\
n_{i}^{t h}(T) & \equiv \frac{g_{i}}{(2 \pi \hbar)^{3}} \int d M d^{3} p e^{-E_{M} / T} \mathcal{A}(M) \\
& =\frac{g_{i} T}{2 \pi^{2} \hbar^{3}} \int d M M^{2} K_{2}(M / T) \mathcal{A}(M)
\end{aligned}
$$


with the box volume $V$, the degeneracy of the species $g_{i}$ and $\mathcal{A}(M)$ as the spectral function. $K_{2}(x)$ is the modified Bessel function (second kind) and $E_{M}^{2}=p^{2}+M^{2}$. The reaction rates i.e. number of reactions per unit volume per unit time for for $2 \leftrightarrow n$ is given by

$$
\begin{aligned}
& \frac{d N_{2 \rightarrow n}}{d^{4} x}=A \lambda_{1} \lambda_{2}, \\
& \frac{d N_{n \rightarrow 2}}{d^{4} x}=A \prod_{j=1}^{n} \lambda_{j},
\end{aligned}
$$

with

$$
\begin{aligned}
A & \equiv \int \frac{g_{1} d^{3} p_{1}}{(2 \pi \hbar)^{3}} \frac{g_{2} d^{3} p_{2}}{(2 \pi \hbar)^{3}} \sigma_{2 \rightarrow n} v_{\text {rel }} e^{-\left(E_{1}+E_{2}\right) / T} \\
& =\left\langle\sigma_{2 \rightarrow n} v_{\text {rel }}\right\rangle n_{1}^{t h}(T) n_{2}^{t h}(T)
\end{aligned}
$$

And, the rates for decay and formation of a $1 \leftrightarrow n$ reaction are

$$
\begin{aligned}
& \frac{d N_{1 \rightarrow n}}{d^{4} x}=A \lambda_{1}, \\
& \frac{d N_{n \rightarrow 1}}{d^{4} x}=A \prod_{j=1}^{n} \lambda_{j},
\end{aligned}
$$

with

$$
\begin{aligned}
A & \equiv \int d M \frac{d^{3} p}{(2 \pi \hbar)^{3}} \frac{M}{E_{M}} \Gamma(M) e^{-E_{M} / T} \mathcal{A}(M) \\
& =\langle\Gamma\rangle n_{1}^{t h}(T)
\end{aligned}
$$

Expressions for $\left\langle\sigma_{2 \rightarrow n} v_{\text {rel }}\right\rangle$ and $\langle\Gamma\rangle$ are found in [4]. Using the equations above, a system of equation can now be deducted for arbitrary systems of degrees of freedom and reactions between them. In the following, the results of [305] and [4] are used. The full sets needed to obtain the analytic results used for comparison are therefore found in the cited references [305] and [4]. One example is given as an illustration: Considering a system of $d, \pi, N$ with two possible $3 \leftrightarrow 2$ reactions: $\pi p n \leftrightarrow \pi d$ and $N p n \leftrightarrow N d$ (the in this work employed $d$ catalysis reactions), the system of rate equation reads as follows.

$$
\begin{cases}n_{d}^{t h} \dot{\lambda_{d}} & =\left(R_{\pi d}+R_{N d}\right)\left(\lambda_{p}^{2}-\lambda_{d}\right) \\ n_{p}^{t h} \dot{\lambda_{N}} & =-\left(R_{\pi d}+R_{N d}\right)\left(\lambda_{p}^{2}-\lambda_{d}\right) \\ \dot{\lambda_{\pi}} & =0 \\ R_{\pi d} & =\left\langle\sigma v_{r e l}\right\rangle_{\pi d} n_{\pi}^{t h} n_{d}^{t h} \lambda_{\pi} \\ R_{N d} & =\left\langle\sigma v_{r e l}\right\rangle_{N d} 2 n_{p}^{t h} n_{d}^{t h} \lambda_{N}\end{cases}
$$

where the $\frac{d \lambda}{d t}$ derivative is abbreviated by $\dot{\lambda}$. The number of $\mathrm{p}$ and $n$ is assumed to be equal and the initial yield needed to solve the system are determined by the box setup. The resulting yields of this example system are found as the dashed line in Figure 5.9.

\section{Binary reactions}

As the first result, Figure 5.2 shows a box calculation with only $\pi$ and $\rho$ mesons. The only included reaction in the calculation is $\rho \leftrightarrow \pi \pi$. 

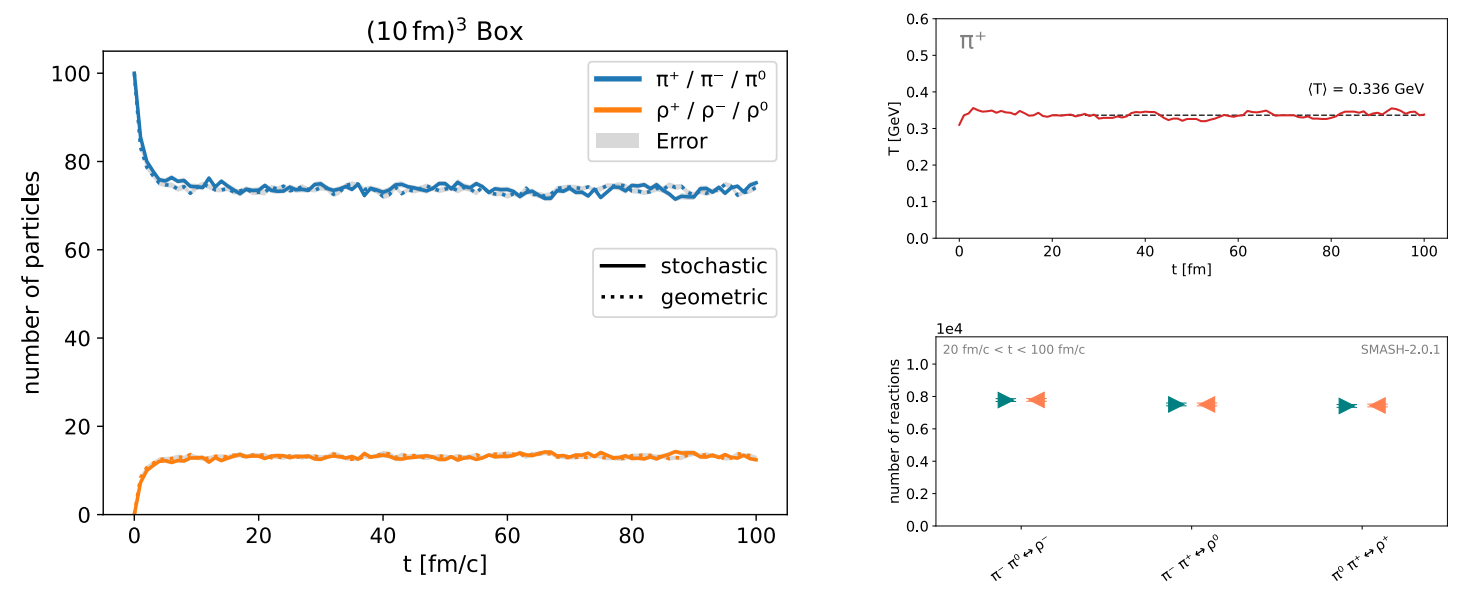

Figure 5.2: Box of $\rho$ and $\pi$ mesons. Left: Time evolution of particle numbers. Upper right: Temperature time evolution. Lower right: Number of forward and backward reactions after equilibration.

Since in the following the results are presented in a similar fashion, first a few general explanations on Figure 5.2 are given: On the left-hand side, the number of particles over time is shown. The lines being averages of the different isospin types if listed together in the legend. Generally, the results employ the stochastic criterion. Only on the multiplicity plot, the same calculation employing the geometric criterion is shown for comparison as the dotted line. Error bands are plotted in grey behind the lines. On the upper right, the time evolution of the temperature is shown, which is obtained, as described above, by fitting the $\pi^{+}$energy spectra. The dotted line illustrates the time over which the temperature average is obtained, which is also noted in the plot. The lower right plot lists the number of forward and backward reactions for the different reactions on the x-axis. One triangle shows the number of forward and the one facing in the other direction the number of backward reactions. Forward and backward reactions are counted for the time interval noted in the top left of the plot. In general, the counting of the reactions and the averaging of the temperature is done from a time, where the system can be considered in chemical equilibrium i.e. the number of particles is constant, which can be verified from the multiplicity plot on the left. Note that counting forward and backward reactions is necessary to verify that detailed balance is conserved. Chemical equilibrium alone is not sufficient since it is also possible with unbalance, cyclic reactions. Together this set of plots allows to check if and how the system equilibrates and whether detailed balance is conserved for all present reactions.

Coming back to the results from Figure 5.2, the number of particles quickly equilibrates with around $15 \rho$ being formed by $\pi \pi$ reactions and is in chemical equilibrium at about 10 $\mathrm{fm}$. The temperature average and reaction counting starts at $20 \mathrm{fm}$, where the system can be safely considered in chemical equilibrium. The temperature in equilibrium is $T=0.336$ $\mathrm{GeV}$ and from the lower right plot it is clear that detailed balance is conserved since all possible isospin combinations of $\rho \rightarrow \pi \pi$ and $\pi \pi \rightarrow \rho$ respectively occur equally often.

The stochastic and the geometric criterion results agree for this box calculation, which presents a first verification that the new treatment of the stochastic criterion is also correct for inelastic 2-body reactions. 

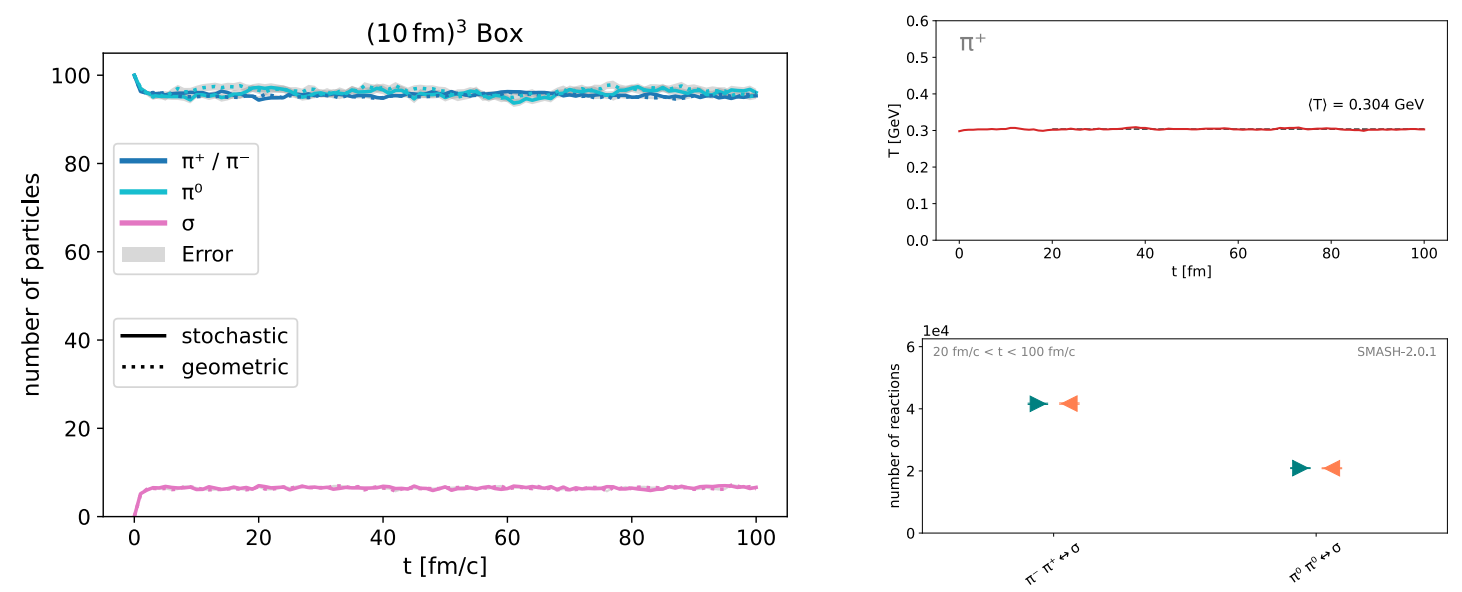

Figure 5.3: Box of $\sigma$ and $\pi$ mesons. Left: Time evolution of particle numbers. Upper right: Temperature time evolution. Lower right: Number of forward and backward reactions after equilibration.

A similar box result in this case with $\sigma \leftrightarrow \pi \pi$ is found in Figure 5.3. Again only one $2 \leftrightarrow 1$ reaction is probed. As seen in the left plot, the system even faster equilibrates since the number of initialized $\pi$ is closer to the equilibrated number and so also the temperature is nearly constant (upper right plot). The number of forward and backward reactions is again equal for the respective isospin combinations, with the total number of $\sigma \leftrightarrow \pi^{0} \pi^{0}$ being half of $\sigma \leftrightarrow \pi^{+} \pi^{0}-$ due to the identical particles $\left(\pi^{0}\right)$ in the 2-body state (causing a symmetry factor of 2 !). The result again confirms that for such simple, one inelastic reaction, systems the results are independent of the chosen collision criterion as the multiplicity evolution in the box matches.

\begin{tabular}{l|cccccc} 
Criterion & $\langle T\rangle[\mathrm{GeV}]$ & $\left\langle N_{\rho}\right\rangle$ & $\left\langle N_{\pi}\right\rangle$ & $R_{\rho \pi}$ & $R_{\rho \pi}^{t h}$ & $\frac{R_{\rho \pi}-R_{\rho \pi}^{t h}}{R_{\rho \pi}^{t h}}$ \\
\hline Stochastic & 0.336 & 13.2 & 73.9 & $2.438 \mathrm{E}-03$ & $2.337 \mathrm{E}-03$ & $\mathbf{0 . 0 4 3}$ \\
Geometric & 0.317 & 13.3 & 73.4 & $2.466 \mathrm{E}-03$ & $2.579 \mathrm{E}-03$ & $\mathbf{0 . 0 4 4}$
\end{tabular}

Table 5.1: For box of $\rho$ and $\pi$ mesons in Figure 5.2. $R_{\rho \pi}=\frac{N_{\rho}}{N_{\pi}^{2}}$.

\begin{tabular}{l|cccccc} 
Criterion & $\langle T\rangle[\mathrm{GeV}]$ & $\left\langle N_{\sigma}\right\rangle$ & $\left\langle N_{\pi}\right\rangle$ & $R_{\sigma \pi}$ & $R_{\sigma \pi}^{t h}$ & $\frac{R_{\sigma \pi}-R_{\sigma \pi}^{t h}}{R_{\sigma \pi}^{t h}}$ \\
\hline Stochastic & 0.304 & 6.5 & 95.5 & $7.136 \mathrm{E}-04$ & $7.650 \mathrm{E}-04$ & $\mathbf{0 . 0 6 7}$ \\
Geometric & 0.303 & 6.5 & 95.3 & $7.141 \mathrm{E}-04$ & $7.690 \mathrm{E}-04$ & $\mathbf{0 . 0 7 1}$
\end{tabular}

Table 5.2: For box of $\sigma$ and $\pi$ mesons in Figure 5.3. $R_{\sigma \pi}=\frac{N_{\sigma}}{N_{\pi}^{2}}$.

Even though both approaches for the collision criterion agree, it is still not clear if the transport approach agrees with the thermal (analytic) expectation. As explained above, one possible verification is to compare with the kinetic theory expectation for the equilibrated multiplicities, where a ratio is taken to cancel the chemical potential specific to the reaction. The relevant values for the $\sigma \leftrightarrow \pi \pi$ and $\rho \leftrightarrow \pi \pi$ boxes are displayed in Table 5.1 and Table 5.2. The multiplicity ratio that is taken is given in the caption. The equilibrated multiplicities are averages fitted over the same range as the temperature, so in the both cases for $20-100 \mathrm{fm}$. $R^{\text {th }}$ is the same ratio with the calculated analytic grand-canonical 
ideal gas expectation according to Equation 5.2 (multiplied by the volume of the box). The comparison is performed in the last column, where the absolute value of the relative difference is displayed. For the precision of the temperature and multiplicity fit that is limited by statistics, a relative difference of less than $10 \%$ can be seen as agreement. Table 5.1 and Table 5.2 therefore show that the equilibrated multiplicities match the analytic expectation.
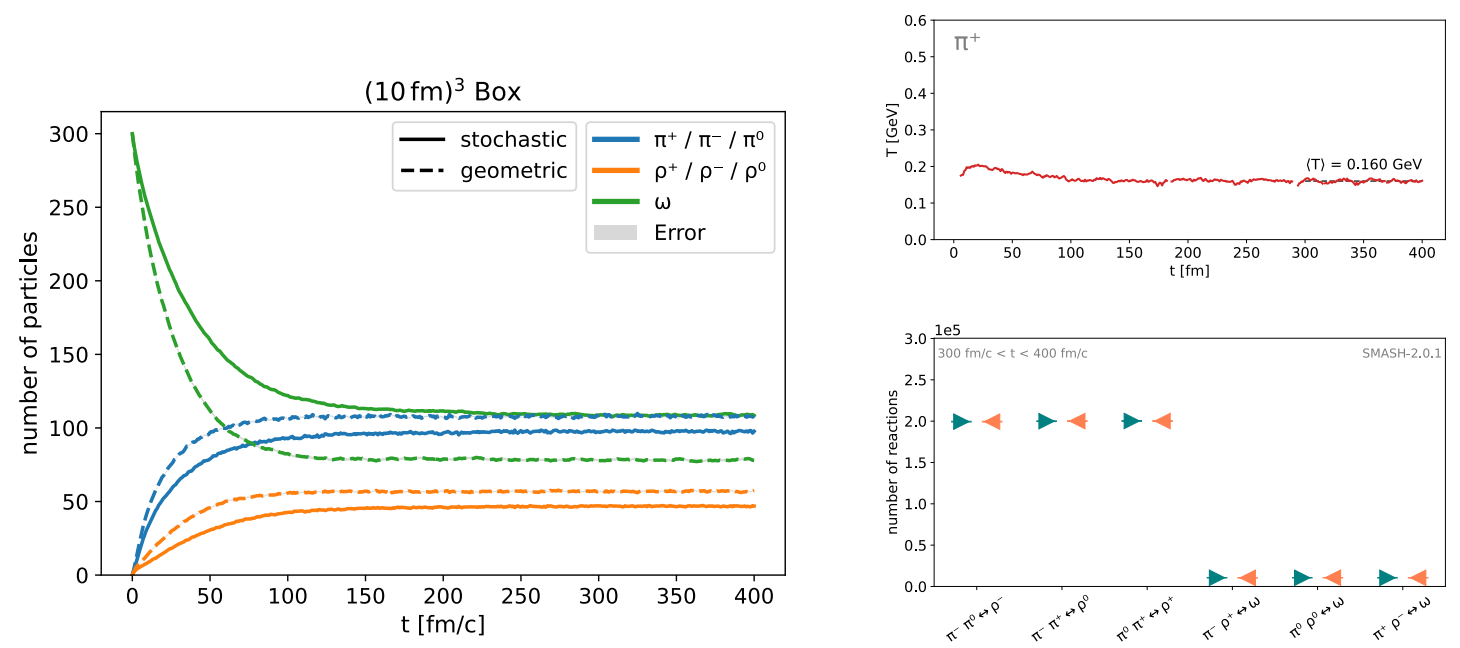

Figure 5.4: Box of $\rho, \omega$ and $\pi$ mesons (without $\omega \rightarrow 2 \pi$ reaction). Left: Time evolution of particle numbers. Upper right: Temperature time evolution. Lower right: Number of forward and backward reactions after equilibration.

The second scenario are two mesonic infinite matter calculations with two reactions included for each. Surprisingly, already for this still simple calculation a disagreement between both collision criterion is observed.

The first box includes $\pi, \rho$ and $\omega$ mesons with two reactions possible between them: $\rho \leftrightarrow \pi \pi$ and $\omega \leftrightarrow \rho \pi$. Note that the usually included $\omega \leftrightarrow \pi \pi$ reaction is neglected for simplicity. The multiplicity and temperature evolution as well as the reaction number comparison for this setup are presented in Figure 5.4. First of all, the results for the stochastic criterion show the system chemical and thermally equilibrating with detailed balance being conserved for all isospin possibilities of both possible reactions. $\rho \leftrightarrow \pi \pi$ being much more frequent than $\omega \leftrightarrow \rho \pi$, since the latter is suppressed by needing a $\rho$ being formed and colliding with another $\pi$ before decaying. Interestingly, the multiplicity time evolution differs for the geometric criterion.

\begin{tabular}{l|ccccccc} 
Criterion & $\langle T\rangle[\mathrm{GeV}]$ & $\left\langle N_{\rho^{0}}\right\rangle$ & $\left\langle N_{\omega}\right\rangle$ & $\left\langle N_{\pi^{+}}\right\rangle$ & $R_{\omega \pi}$ & $R_{\omega \pi}^{t h}$ & $\left|\frac{R_{\omega \pi}-R_{\omega \pi}^{t h}}{R_{\omega \pi}^{t h}}\right|$ \\
\hline Stochastic & 0.160 & 46.8 & 108.7 & 97.6 & $1.170 \mathrm{E}-04$ & $1.140 \mathrm{E}-04$ & $\mathbf{0 . 0 2 6}$ \\
Geometric & 0.148 & 57.0 & 78.2 & 107.9 & $6.222 \mathrm{E}-05$ & $1.441 \mathrm{E}-04$ & $\mathbf{0 . 5 6 8}$
\end{tabular}

Table 5.3: For box of $\rho, \omega$ and $\pi$ mesons in Figure 5.4. $R_{\omega \pi}=\frac{N_{\omega}}{N_{\pi}^{3}}$.

The difference is not only seen in the multiplicity values, but also for the equilibrated multiplicity values as seen on the left plot and confirmed by the averaged values in Table 5.3. The difference being the strongest for the $\omega$. The difference also leads to different temperatures for both boxes. The table shows one of the possible multiplicity ratios $R_{\omega \pi}=\frac{N_{\omega}}{N_{\pi}^{3}}$, 
where the chemical ratio specific to the present reactions cancels. It is clear that the equilibrated multiplicity ratio for the geometric criterion differs from the analytic expectation, even though the different temperature does mean a different expected ratio. The stochastic criterion meanwhile matches the analytic expectation perfectly.
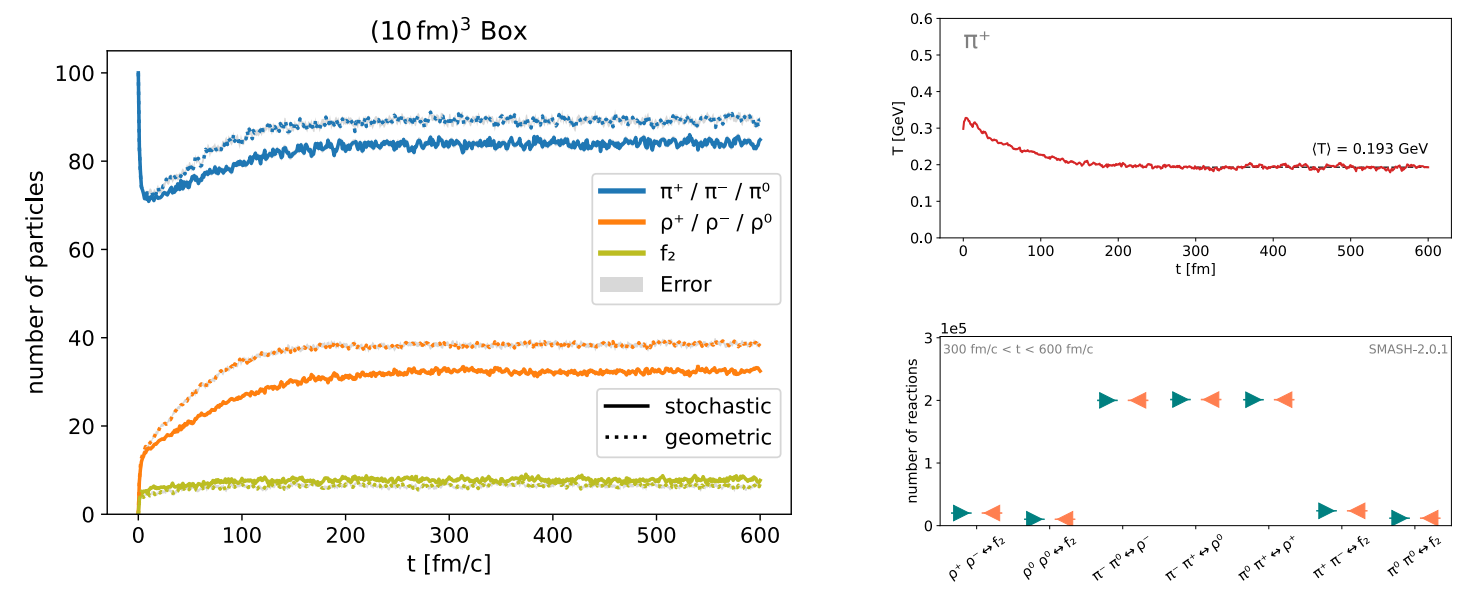

Figure 5.5: Box of $\rho, f_{2}$ and $\pi$ mesons. Left: Time evolution of particle numbers. Upper right: Temperature time evolution. Lower right: Number of forward and backward reactions after equilibration.

These findings are confirmed by the second two-reaction box calculation, which includes the hadrons $\pi, \rho$ and $f_{2}$, which are interacting via $\rho \leftrightarrow \pi \pi$ and $f_{2} \leftrightarrow \rho \rho$. The results in Figure 5.5 show again the system equilibrating with detailed balance being conserved for all reactions for the stochastic criterion. While detailed balance being also conserved for the geometric criterion (not shown), again the multiplicity evolution differs. Similar as for the other 3-particle box, 5 he values for the ratio $R_{f_{2} \rho}=\frac{N_{f_{2}}}{N_{\rho}^{2}}$ of the equilibrated multiplicities in Table 5.4 differ again for the geometric criterion from the analytic expectation. Whereas, the stochastic criterion results agree with it.

\begin{tabular}{l|ccccccc} 
Criterion & $\langle T\rangle[\mathrm{GeV}]$ & $\left\langle N_{\rho^{0}}\right\rangle$ & $\left\langle N_{f_{2}}\right\rangle$ & $\left\langle N_{\pi^{+}}\right\rangle$ & $R_{f_{2} \rho}$ & $R_{f_{2} \rho}^{t h}$ & $\frac{R_{f_{2} \rho}-R_{f_{2} \rho}^{t h}}{R_{f_{2} \rho}^{t h}}$ \\
\hline Stochastic & 0.193 & 32.5 & 7.8 & 83.8 & $7.404 \mathrm{E}-03$ & $7.187 \mathrm{E}-03$ & $\mathbf{0 . 0 3 0}$ \\
Geometric & 0.168 & 38.4 & 6.5 & 89.1 & $4.403 \mathrm{E}-03$ & $1.181 \mathrm{E}-02$ & $\mathbf{0 . 6 2 7}$
\end{tabular}

Table 5.4: For box of $\rho, f_{2}$ and $\pi$ mesons in Figure 5.5. $R_{f_{2} \rho}=\frac{N_{f_{2}}}{N_{\rho}^{2}}$.

Since the above results for all mesonic boxes match the analytic expectation for the ratios of multiplicities, the verification for the inelastic two-body reactions is successful. The stochastic criterion is generally an improvement compared to the geometric criterion result regarding the agreement with the analytic results. This is expected since the theoretical foundation of the geometric criterion is not as rigorous. Even though slightly surprising that a difference between both criteria is already apparent for such simple systems, the studied systems are specific test scenarios. It is unclear how relevant the differences are in more realistic, dynamic scenarios like a heavy-ion collision. A more detailed study on why the geometric criterion differs from the correct benchmark results obtained with the stochastic criterion is left for future studies and beyond the scope of the verification of the introduced stochastic criterion. 


\section{3-to-1 reactions}

The first result for the more interesting, newly-introduced multi-particle reactions is presented in Figure 5.6. Here and in the following, no direct comparison to the geometric

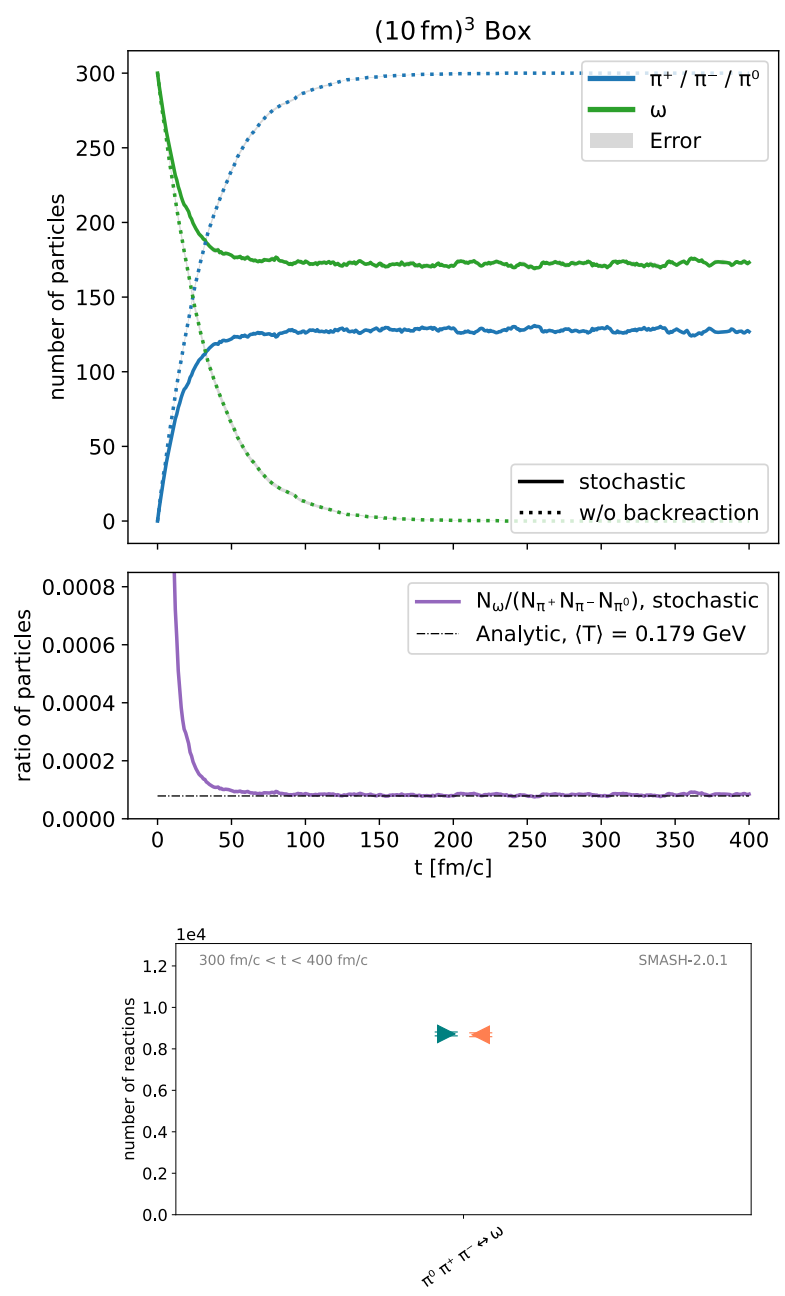

Figure 5.6: Box of $\omega$ and $\pi$ mesons interacting via $\omega \leftrightarrow \pi^{0} \pi^{+} \pi^{-}$. Top: Time evolution of multiplicities. Middle: Time evolution of multiplicity ratio. Bottom: Number of forward and backward reactions after equilibration.

criterion is possible anymore since it cannot be employed for multi-particle reactions. The box calculation only includes the $\omega \leftrightarrow \pi^{0} \pi^{+} \pi^{-}$reaction and $\omega$ and $\pi$ as the only two particle species. Previously SMASH only could describe the $1 \rightarrow 3$ decay. If no back-reaction is included (dotted line), which was so far the only option, the $\omega$ mesons naturally are observed to only dissipate over time without reaching an equilibrium state. With the stochastic reaction, it is possible to account for the 3-body back-reaction to reach equilibrium and detailed balance for $\omega \leftrightarrow \pi^{0} \pi^{+} \pi^{-}$. Figure 5.6 shows on the top plot that chemical equilibration of the system of $\omega$ and $\pi$ is reached in this case. The middle plot summarizes the information previously displayed in tables by showing the time evolution of the relevant ratio compared to the analytic result for the fitted equilibration temperature (here $\langle T\rangle=0.179 \mathrm{GeV}$ ). Since the ratio matches the analytic value over time, also the equilibrated multiplicity produced by the $\omega \leftrightarrow \pi^{0} \pi^{+} \pi^{-}$reaction is verified. Most importantly, the bottom plot shows that detailed balance is conserved for the first time 
for a multi-particle reaction directly in SMASH.
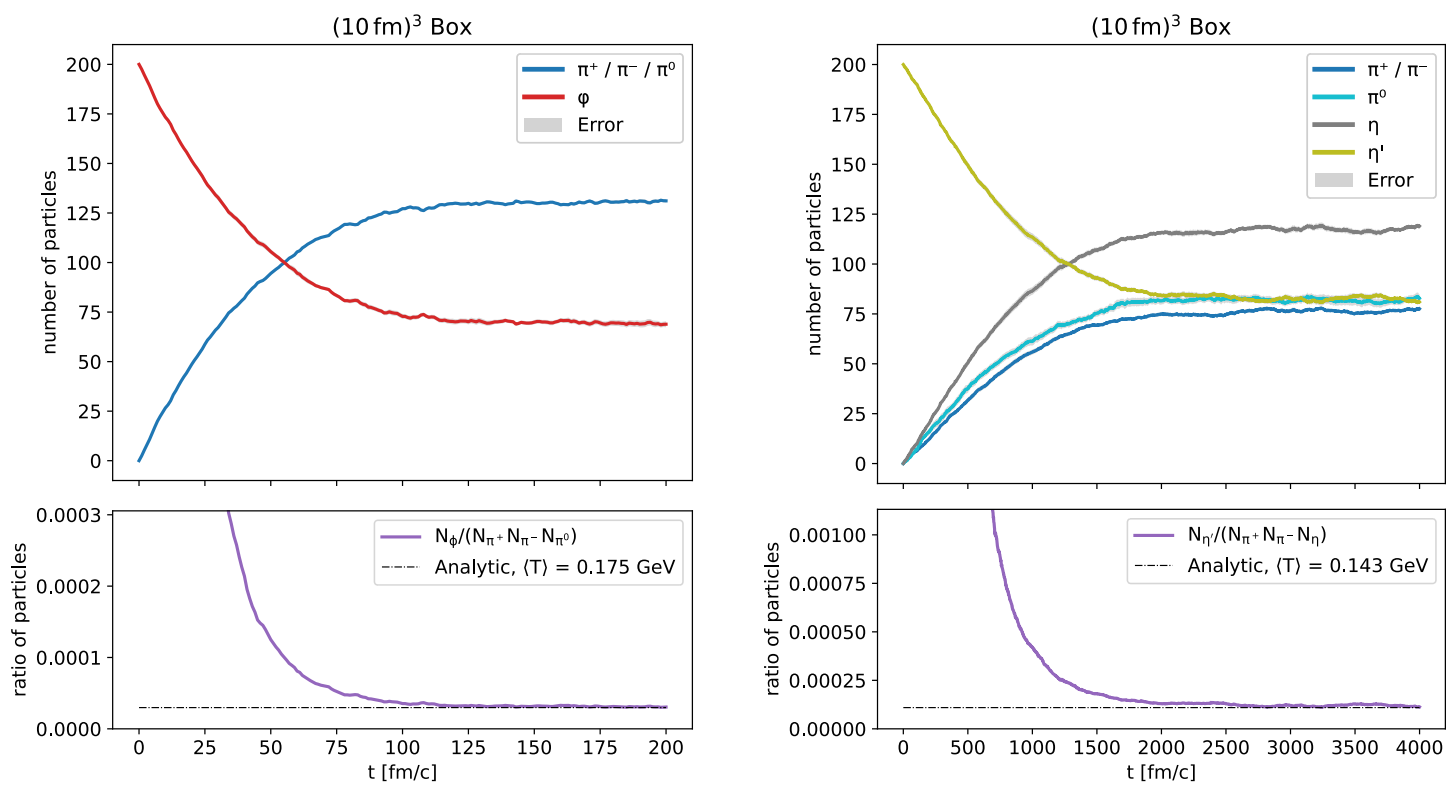

Figure 5.7: Left: Box of $\phi$ and $\pi$ mesons interacting via $\phi \leftrightarrow \pi^{0} \pi^{+} \pi^{-}$. Right: Box of $\eta^{\prime}, \eta$ and $\pi$ mesons interacting via $\eta^{\prime} \leftrightarrow \eta \pi \pi$. For both: Time evolution of multiplicities (top) and of multiplicity ratios (bottom).

The results for 3-to-1 reactions are complemented with Figure 5.7, which shows the box calculation results for the other 3 -to-1 reactions discussed in this work. On the left, the result for $\phi \leftrightarrow \pi^{0} \pi^{+} \pi^{-}$is displayed for a box with only $\phi$ and $\pi$. On the right, a box with $\eta, \eta^{\prime}$ and $\pi$ for the reactions $\eta^{\prime} \rightarrow \eta \pi \pi$. Note that the isospin asymmetry for the $\pi$ is caused by having $\eta \pi^{+} \pi^{-}$and $\eta \pi^{0} \pi^{0}$ as the two possible 3-body states, with the latter having two identical particles. For both decay back-reactions of $\phi$ and $\eta^{\prime}$, the system equilibrates and detailed balance is conserved for all $3 \leftrightarrow 1$ reactions (plot for the latter not shown). The ratios of the equilibrated multiplicities again match the analytic expectation for the temperature of the box.

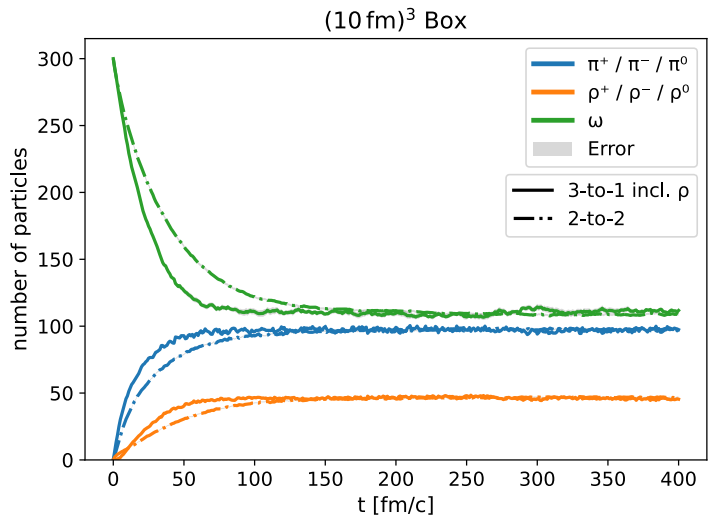

Figure 5.8: Time evolution of multiplicities for a box of $\rho, \omega$ and $\pi$ mesons. Comparison between direct 3-to-1 and two-step 2-body reaction chain treatment for $\omega \leftrightarrow \pi \pi \pi$. Both results employ the stochastic criterion. 
One interesting comparison already possible with the so-far presented, is to compare the result for the direct $\omega 3$-to- 1 reaction (Figure 5.6) with the result modeling same reaction with a 2-body reaction chain (Figure 5.4). Because of the limitation of the geometric criterion of only being applicable for 2-body reaction, it is common to emulate multi-particle reaction with 2-body reaction chains where each step still conserves detailed balance. One of the main advantages of the stochastic criterion is that this emulation is not necessary. It, therefore, enables to constrain the effect of these 2-body reaction chains on the results, which will be done in this work on multiple occasions.

The first comparison of this kind is shown in Figure 5.8. The direct 3-to-1 reaction $\omega \leftrightarrow \pi \pi \pi$ is compared with the two-step 2-body reaction chain $\omega \leftrightarrow \rho \pi \leftrightarrow \pi \pi \pi$ emulating the same reaction. Note that for the $\omega$ reaction specifically, it might be physical that both the direct and the two-step reaction are possible. Still, for this case, both are strictly distinguished to study the maximum effect of employing either treatment. Both results are obtained with the stochastic criterion, but only the solid line includes the $\omega \leftrightarrow \pi \pi \pi$ reaction. The result is similar to the setup in Figure 5.6, but includes the $\rho \leftrightarrow \pi \pi$ reaction to allow for a more direct comparison to the reaction chain approach, which necessarily includes it. Interestingly, the multiplicity evolution is not matching. The multiplicities equilibrate faster in the direct 3-to-1 case. This is explained by the fact that for multiparticle reactions, more particles are reacting at once. In addition, a number of $\rho$ mesons decay before they react with $\pi$ to an $\omega$, which effectively slows down the equilibration further in the 2-to-2 case. This difference in equilibration time is one of the main differences observed in this work between multi-particle reactions and 2-body reaction chains and is also by the rate equations as seen below.

Two more notes on the result shown in Figure 5.8: First, the extracted temperature is the same for both treatments and also the equilibrated multiplicities matches the analytic expectation for both cases, which underlines the need to probe the time evolution analytically as done with rate equations in the following. Second, comparing the equilibration time for the system with just the $\omega \leftrightarrow \pi \pi \pi$ reaction in Figure 5.6 (around $50 \mathrm{fm}$ ) with the equilibration time in Figure 5.8 or Figure 5.4 (around $150 \mathrm{fm}$ ), it is clear that just equilibrating the one 3 -to- 1 reaction is much faster. This is a trivial finding, since more reactions need more time to equilibrate. But for the 3 -to- 1 case, the $\rho \leftrightarrow \pi \pi$ reaction can just be neglected since it was only added for the sake of the comparison and, in a sense, artificially slowed the equilibration down. In contrast, $\rho \leftrightarrow \pi \pi$ is essential in mediating the same reaction in the 2-to- 2 case. Therefore, the equilibration time when just considering the $\omega \leftrightarrow \pi \pi \pi$ reaction in a box is by construction even large between the two approaches then observed in Figure 5.8.

\section{3-to-2 reactions}

The next class of multi-particle reactions discussed are 3-to-2 reactions for deuterons. The two reactions are $\pi n p \leftrightarrow \pi d$ and $N n p \leftrightarrow N d$, which are known to be relevant for $d$ formation and break-up. Figure 5.9 displays the evolution of a box scenario initialized with $\pi$ and $N$ including both reactions and shows deuterons forming over time. The included $\pi d \leftrightarrow N N$ reactions are neglected for the presented calculation since it equilibrates much slower due to its small cross-section, making it less relevant. The figure includes five different time evolutions. The colored lines show the time evolution of the transport approach. All multiplicity evolutions show that the system equilibrates (with the $\pi$ multiplicity constant since the $\pi$ is always the same in the incoming and outgoing state). That detailed balance is conserved after equilibration was checked separately. Three different treatments 


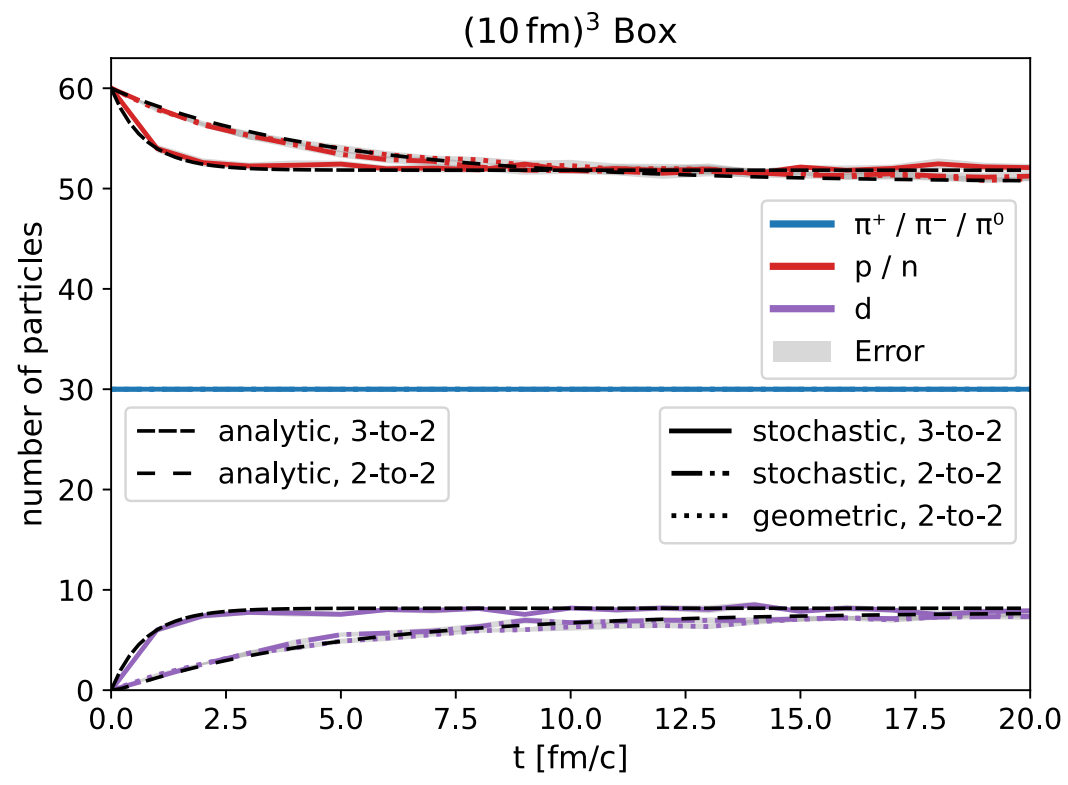

Figure 5.9: Time evolution of multiplicities for a box of $d, N$ and $\pi$. Comparison between different treatments of $\pi n p \leftrightarrow \pi d$ and $N n p \leftrightarrow N d$. Analytic result obtained with rate equations [4].

of $\pi n p \leftrightarrow \pi d$ and $N n p \leftrightarrow N d$ are distinguished in the plot. The stochastic 3-to-2 case that directly performs the reactions via the stochastic criterion is most relevant to this work. The geometric criterion relies on a 2-body reaction chain via a fake $d^{\prime}$ resonance $\left(\{\pi, N\} n p \leftrightarrow\{\pi, N\} d^{\prime} \leftrightarrow\{\pi, N\} d\right)$ as already explained before (Section 4.3.2). The result for the 2-to-2 treatment is shown for the stochastic and geometric criterion. The 2-to-2 result is invariant under a change of the criterion.

However, interestingly there is a difference between the 3-to-2 and 2-to-2 treatment. The multi-particle reaction clearly equilibrates the medium faster, which is the same finding as in Figure 5.8. Moreover, Figure 5.9 also shows that this is analytically expected. The above-described rate equations (thinner black lines ) also show the medium faster equilibrating in the 3-to- 2 case. The analytic result agrees perfectly with the transport calculation validating the implementation of the stochastic multi-particle reactions again, in this case for the deuteron 3 -to- 2 reactions. The validation is even more explicit since, if available, the rate equation also validates the dynamical equilibration process over time as well as the found difference between the different treatments. The earlier comparison, in contrast, only checked the static number of particles after equilibration.

\section{5-to-2 reactions}

5-to-2 reactions are the multi-particle reaction class remaining to be discussed. This is the first time that 5-body reactions are treated directly in transport approaches. The application for this reaction class are $N \bar{N}$ annihilation reactions that are known to produce $5 \pi$ on average. The relevance of the $5 \pi$ annihilation back-reaction is a long-standing open question addressed in the last section of this chapter. Here, it is first verified that the equilibration properties of the 5-to- 2 reaction are correctly described. Figure 5.10a shows the multiplicities of $p$ and $\pi$ rapidly equilibrating due to them interacting directly via 


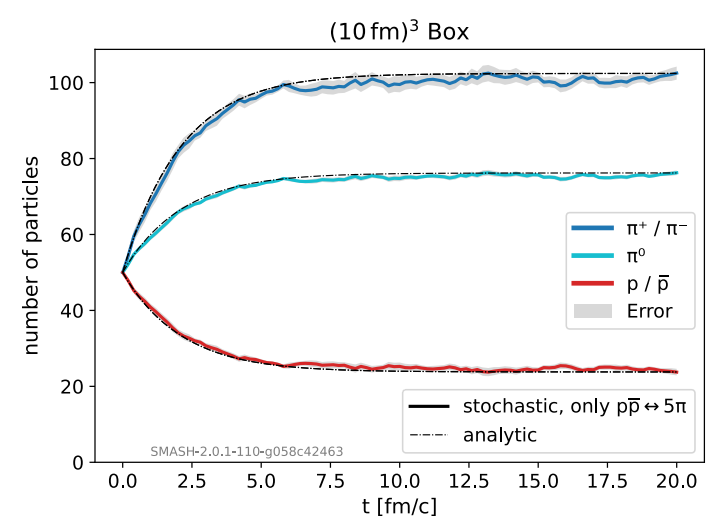

(a) Stochastic 5-to-2 approach (only $p \bar{p} \leftrightarrow 5 \pi$ )

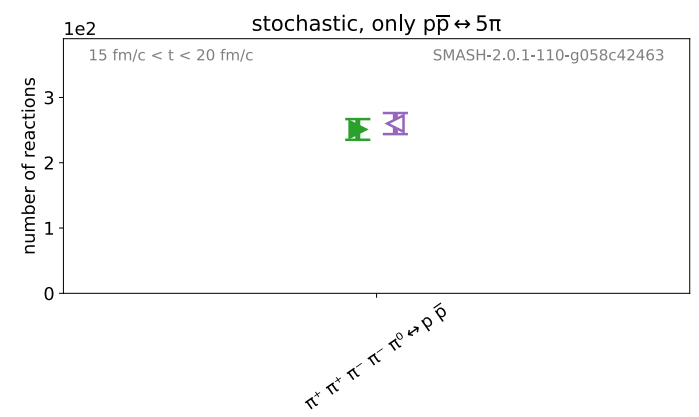

(c) Reaction numbers (Stochastic 5-to-2 approach (only $p \bar{p} \leftrightarrow 5 \pi$ ))

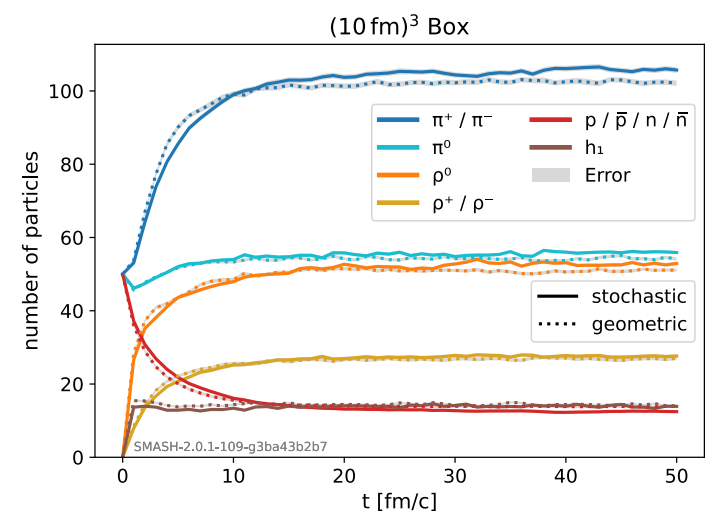

(b) Resonance approach

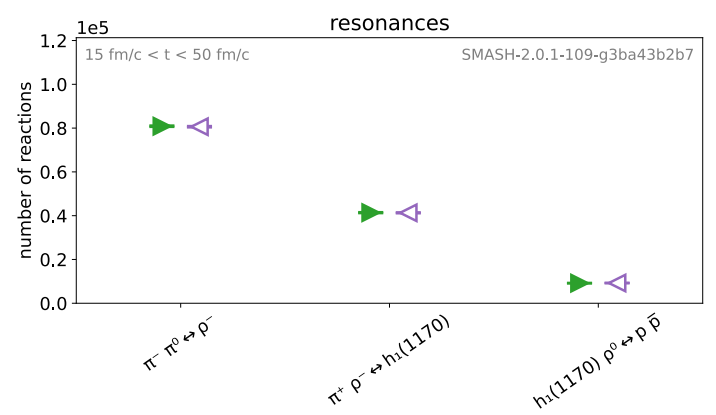

(d) Reaction numbers (Resonance approach, geometric)

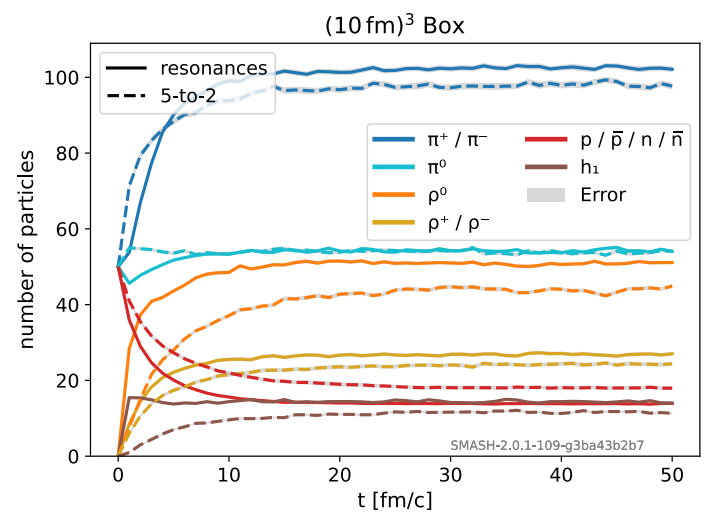

(e) Stochastic 5-to-2 (incl. $\rho$ and $h_{1}$ in box) vs. resonance approach

Figure 5.10: Time evolution of multiplicities and reaction numbers for box calculations including different approaches for $N \bar{N} \leftrightarrow 5 \pi$ reactions. Analytic rate equation result from [305]. 
$p \bar{p} \leftrightarrow 5 \pi$. This result is naturally obtained with the stochastic criterion as the only criterion able to employ multi-particle reactions (stochastic 5-to-2 approach). The $n \bar{n} \leftrightarrow$ $5 \pi$ reactions is neglected for this calculation, in order to compare to the analytic calculation from [305], which only includes the $p$ reaction. The analytic calculation in Figure 5.10a is again obtained with rate equations and agrees with the 5-to-2 transport calculation, thereby verifying the equilibration properties of the stochastic 5-to-2 treatment. More importantly, then the observed chemical equilibration is the fact that detailed balance is fulfilled. As observed in Figure 5.10c, the number of forward and backward reactions are indeed the same.

Since the transport approach with the geometric criterion is limited to 2-body reactions, a different construct to model $N \bar{N} \leftrightarrow 5 \pi$ is also employed (Section 4.3.3). The 5 -to- 2 reaction is modeled as a chain of 2 -body reactions, in this case involving the two mesonic resonances $\rho$ and $h_{1}$ (hence this approach is referred to as resonance approach): $N \bar{N} \leftrightarrow \rho h_{1} \leftrightarrow 3 \pi \rho \leftrightarrow 5 \pi$. The geometric (and stochastic) criterion is able to conserve detailed balance for every 2-body reaction in this chain, and therefore, detailed balance is also conserved overall. Figure 5.10b shows that employing the stochastic and geometric criterion leads to a similar multiplicity evolution. The system to realize the reaction chain is more complex in comparison to the simpler system in Figure 5.10a for the stochastic 5to-2 approach. Also, the time to equilibrate the simpler system with the stochastic 5 -to-2 approach in Figure 5.10a is much shorter than the system for the resonance approach in Figure 5.10b. Note that a comparison between both results in terms of multiplicity is not possible, since one cannot decide how the intermediate states $\rho$ and $h_{1}$ contribute to either the $\pi$ yield via decays or the $N$ yield via annihilation. Figure 5.10d shows that all three participating reactions are also fulfilling detailed balance once the system is in chemical equilibrium. Only one isospin combination example with the geometric criterion is shown for legibility. Detailed balance is also fulfilled for the other isospin combinations and when the stochastic criterion is employed for the resonance approach (not shown).

To further compare the the two approaches, Figure 5.10e presents a direct comparison between the resonance approach (same as in Figure 5.10b) and the 5-to-2 approach including $\rho$ and $h_{1}$ in the box calculation additionally. This comparison has the same degrees of freedom, but different interactions. With the interactions $\rho \leftrightarrow \pi \pi$ and $h_{1} \leftrightarrow \pi \rho$ being part the same for both results and the annihilation being different: either via $N \bar{N} \leftrightarrow 5 \pi$ or via $N \bar{N} \leftrightarrow h_{1} \rho$. The idea is to produce a fairer comparison between the two approaches in terms of the degrees of freedom than comparing Figure 5.10a and Figure 5.10b. The result is that the time to equilibrate in Figure 5.10e is not as clearly different for the two approaches in this more complex system. For the stochastic 5-to-2 approach, the $\pi$ multiplicity is faster to equilibrate, while the other multiplicities equilibrate at the same rate. In particular, the $h_{1}$ appears to hinder the equilibration of the system. Overall, both comparisons (in Figure 5.10e or between Figure 5.10c and Figure 5.10b) are valid and highlight different aspects: The $N \bar{N} \leftrightarrow 5 \pi$ reaction in the 5 -to- 2 approach does equilibrate much faster than employing a 2-body reaction chain, but this difference in a more complex system with more reactions it seems to be weakened. How the difference in equilibration finding is reflected in a dynamic heavy-ion collision is studied in Section 5.4.

The main difference between the 5-to-2 and the resonance approach is the presence of intermediate states that propagate the medium for a certain (life) time. In a previous study [204], interesting results were achieved by scaling the lifetime, therefore removing the propagation of resonances through the medium and making the (decay) reaction happening instantaneously. In [204], the lifetime of intermediate resonance and with this the reaction 


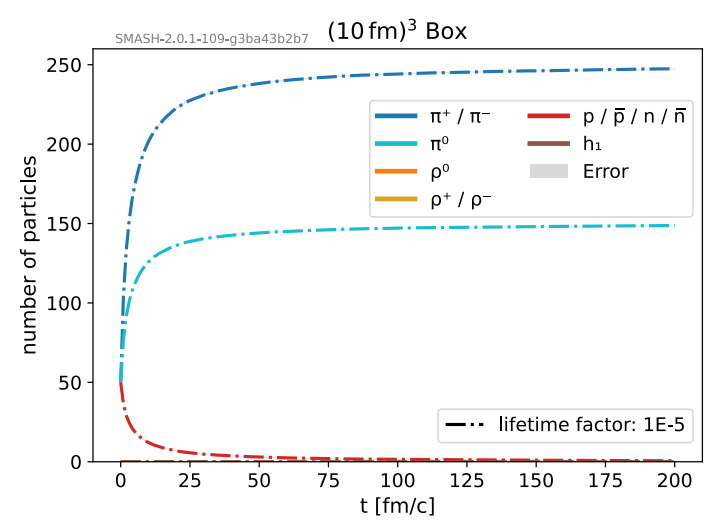

(a) Lifetime scaled with a factor of $10^{-5}$.

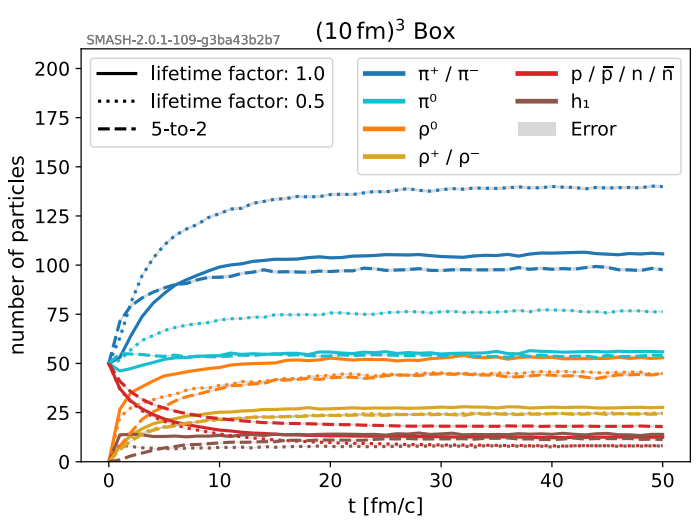

(b) Comparison to halved lifetime.

Figure 5.11: Time evolution of multiplicities for box including $N \bar{N} \leftrightarrow 5 \pi$ reaction via resonances with scaled lifetimes.

timescale is found to have a significant effect on transport coefficients, namely the shear viscosity. Previously disagreeing results on transport coefficients are compatible when the reaction timescale is scaled to be the same. A similar idea is explored in Figure 5.11, where the lifetimes are scaled to explore the effect on the difference between the 5-to-2 and resonance approach. The lifetime is practically set to zero by scaling it with $10^{-5}$ (left plot) and halved by scaling it with 0.5 (right plot, dotted line). However, it appears that achieving a better agreement between the direct 5-to-2 approach and the resonance approach with instantaneous reaction $(\approx 0$ lifetime $)$ is not possible. Since resonances directly decay, no secondary reaction between resonances can occur (including $\rho h_{1}$ ). So, as the left of Figure 5.11 reveals, no new $N \bar{N}$ pairs are formed and they annihilate until none are left. The agreement also is worse for the results with halved lifetimes, displayed in the right plot. Even though an equilibrium for the $N, \bar{N}$ yield is reached for this result, this equilibrated yield (compared to the resonance result with normal lifetimes) is not closer to the yield obtained by the 5-to-2 approach. Therefore, the lifetime shortening of the intermediate resonances in the reaction chain has not the same effect as the multiparticle reactions here. The reaction timescale is inherently different. This makes a comparison of the effect of both treatments, especially the multi-particle reactions, on transport coefficients like the shear viscosity (extending previous studies like [207, 205] and [204]) in the future, particularly interesting, because of the found effect of reaction timescale on them.

The main purpose of the box results above ${ }^{1}$ is to verify the implementation of the stochastic criterion for inelastic two-body and multi-particle reactions in the employed transport approach. The results of this section are summarized as follows:

The treatment of the stochastic criterion in general and the newly introduced multi-particle reactions, in particular, are validated by the results. As mentioned, due to the stochastic rates being rigorously based on the Boltzmann equation, the described setups provide a simple testing ground for the criterion since analytic expectations for the multiplicities (ratios) can be obtained. The analytic expectation for the equilibrated multiplicity ratios is met for all cases with the stochastic criterion. Also, the dynamic equilibration

\footnotetext{
${ }^{1}$ Note that most of the results presented in this section can directly be reproduced by detailed balance tests included in the SMASH analysis suite [225].
} 
process expectation is perfectly matched, where rate equations are available. As shown, the stochastic criterion results are an improvement compared to the geometric criterion for binary reactions, regarding the agreement with the analytic results. Multi-particle back-reactions for decay and scattering processes with 3 or 5 outgoing particles conserve detailed balance in equilibrium. The agreement with the analytic expectation also verifies the derived and implemented probability formulas, including the employed symmetry and degeneracy factors. Furthermore, it is shown that multi-particle reactions equilibrate the system faster than 2-body reaction chains used to model the same reaction. It is interesting to study the implication of this difference for dynamic non-equilibrium nucleus-nucleus systems, also to constrain the effect of a reaction chain on results obtained so far. In general, based on the verification of the equilibrium properties of stochastic criterion and multi-particle reactions in this section, it is possible to explore physically more interesting and realistic non-equilibrium scenarios in the following.

\subsubsection{Collision rate for an expanding sphere}

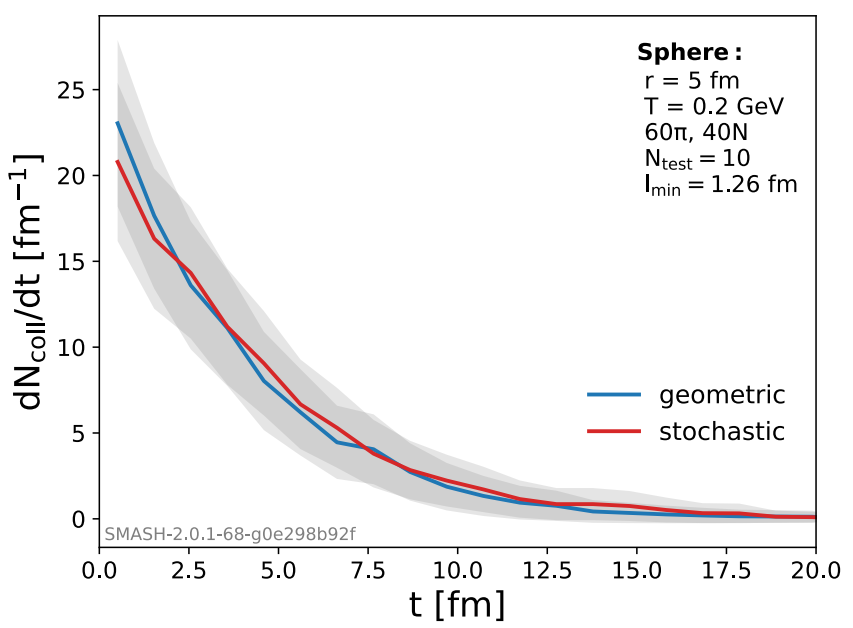

Figure 5.12: Collision rate for the stochastic and geometric collision criterion in an expanding sphere scenario.

After studying static infinite matter calculations, this section starts to explore the more dynamic situation of an expanding sphere (as detailed in Section 2.3). This system is evolving out of equilibrium since it is rapidly diluting due to its expansion. Therefore, the calculation is physically closer to the medium created in a heavy-ion collision but still simple enough to serve as a testing ground for the stochastic approach.

The sphere is initialized ${ }^{2}$ with 100 particles, $60 \pi$ and $40 N$ with the same number of particles for each isospin state. It has a radius of $r=5 \mathrm{fm}$ and expands due to the set temperature of $T=200 \mathrm{MeV}$.

Figure 5.12 shows the result for the collision rate in the case of the geometric (blue line) and stochastic (red line) criterion for the described initial condition. In this case, no multi-particle reactions were employed to only compare the two different approaches for 2-body collisions. As seen in the figure, the collision rate agrees for both collision criteria. This result further validates the stochastic approach for finding collision since no effect of

\footnotetext{
${ }^{2}$ see Section 2.3 for more details on the initial condition of the sphere.
} 
the employed collision criterion is expected in the simple sphere system. Note, however, that the collision rate for the stochastic criterion does depend on the (proper) choice of cell size and test particle number (here $l_{\min }=1.26 \mathrm{fm}$ and $N_{\text {test }}=10$ ) as discussed in Section 5.1.5 below (cf. Figure 5.23).

\subsubsection{Particle yields and $\left\langle p_{T}\right\rangle$ in nucleus-nucleus collisions}

After all, the aim of hadronic transport approaches is the description of heavy-ion collisions. Even though the collision criterion is fundamental to the microscopic of the approach, and therefore it is expected that observables exhibit some sensitivity to its choice, basal observables should ideally not be sensitive to the choice of criterion. Thus, the particle production in AuAu collisions at different $\sqrt{s}$ energies is probed in this section. Again, only two-body reactions are included in the calculations to ensure comparability to study the choice of criteria.

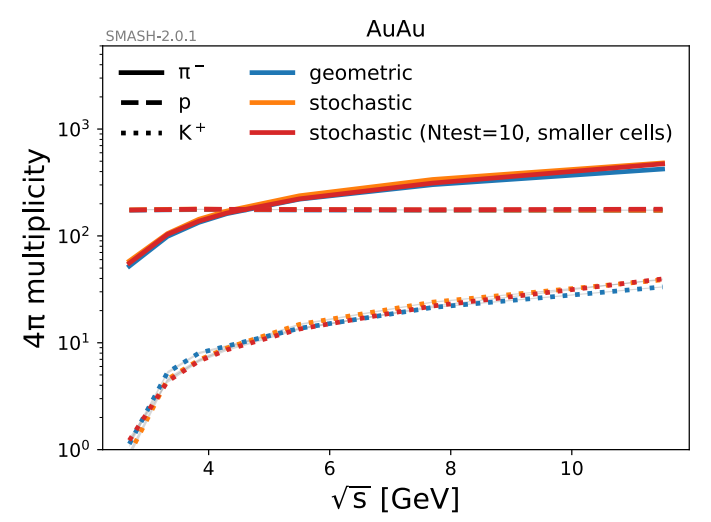

(a)

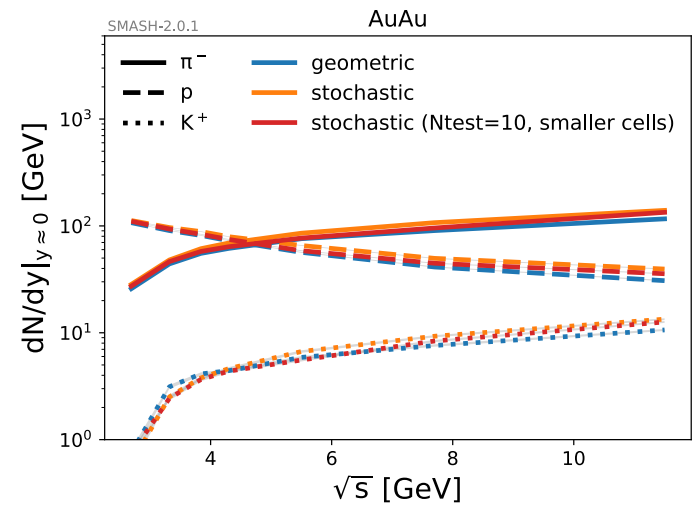

(b)

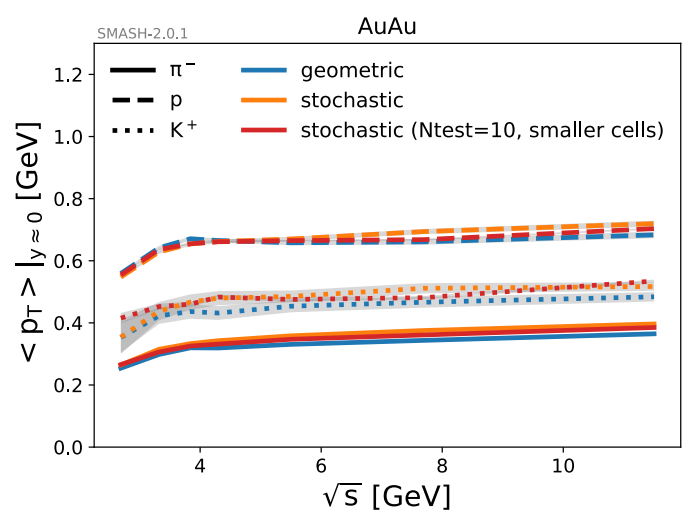

(c)

Figure 5.13: Multiplicity (with and without mid-rapidity cut) and mean transverse momentum for AuAu collisions at different $\sqrt{s}$ energies for stochastic and geometric criterion. No multi-body reactions included.

Figure 5.13 shows the comparison of calculating the multiplicity and mean transverse momentum, $\left\langle p_{T}\right\rangle$, for three abundant, stable particles $\pi, K, N$ for the geometric (cf. Figure 3.1) and stochastic criterion. The multiplicity is the main observable for particle production and $\left\langle p_{T}\right\rangle$ the most basic observable of measuring the collision dynamics in the plane transverse to the beam axis. Stochastic and geometric criteria are found to be 
compatible for the probed low to intermediate beam energy range. Small differences are only observed for the largest energies. Differences in multiplicity are slightly enhanced at mid-rapidity, which is expected since there most collisions take place. All differences are found to minimize by choosing a more appropriate set of simulation parameters for the stochastic criterion (red lines), namely more test particles and smaller grid cells (see the following Section 5.1.5 for details). The here found independence from the choice of criteria for basal observables also further validates the treatment of stochastic rates in SMASH.

Note that the stochastic criterion is not applicable for describing the very dilute system in elementary collisions ${ }^{3}$. Therefore, no such result is presented.

\subsubsection{Numerical stability of results with the stochastic criterion}

Recalling the different probability formulas in Section 4.1 and especially the most general probability formulation in Eq. 4.32

$$
P_{12 \ldots n \rightarrow 1^{\prime} 2^{\prime} \ldots m^{\prime}}=\frac{1}{2^{n} \prod_{j=1}^{n} E_{j}} I_{n m} \frac{\Delta t}{\left(\Delta^{3} x\right)^{n-1}},
$$

it is apparent that the choice of $\Delta t$ and $\Delta^{3} x$ affects the calculated collision probability. In the numeric application, $\Delta t$ represents the timestep of the evolution and $\Delta^{3} x$ the subvolume, where particles might interact, which is the cell volume of a spacial grid. Numerically, both are essentially free parameters. To use $P_{12 \ldots n \rightarrow 1^{\prime} 2^{\prime} \ldots m^{\prime}}$ as the probability for a collision decision in a transport approach, the pair of $\Delta t$ and $\Delta^{3} x$ has to be chosen appropriately such that $P \leq 1$. So already from the equation for the collision probability, it is apparent that the combination of timestep $\Delta t$ and subvolume $\Delta^{3} x$, and the stability of the results under changes of both, need to be considered for the stochastic collision criterion.

Even though the following results are predominantly technical, they are essential to understand as prerequisites to probe physically more interesting systems, particularly since realizing suitable numerical conditions for stochastic rates is known to be challenging. The following dependencies related to the subvolume $\Delta^{3} x$ i.e. the cell size of the grid and the timestep size $\Delta t$ are numerical artifacts of the implementation of the stochastic criterion and not of the underlying equations. After all, these artifacts arise, since the theoretical equations only provide the exact solution of the Boltzmann equation for infinitesimal quantities, i.e. for the limits $\Delta^{3} x \rightarrow 0$ and $\Delta t \rightarrow 0$ (as well as for the number of test particles $N_{\text {test }} \rightarrow \infty$ ). These limits can only be approximated numerically. In other words, the cell volume and timestep should be as small as possible and test particle numbers as large as possible from a purely theoretical point of view. But, this theoretical requirement has to be balanced with the simulation runtime simultaneously, which increases for smaller (and therefore more) cells or timesteps and more test particles.

\section{Timesteps}

As mentioned, employing the formula for the collision probability recited in Equation 5.13 only leads to an exact solution of the Boltzmann equation for infinitesimal $\Delta t$. Numerically this limit is only approximated for a transport approach, where the evolution is split into

\footnotetext{
${ }^{3}$ In fact, the stochastic criterion in SMASH is not at all possible to employ for elementary collisions (technically the grid and all cells have no volume).
} 


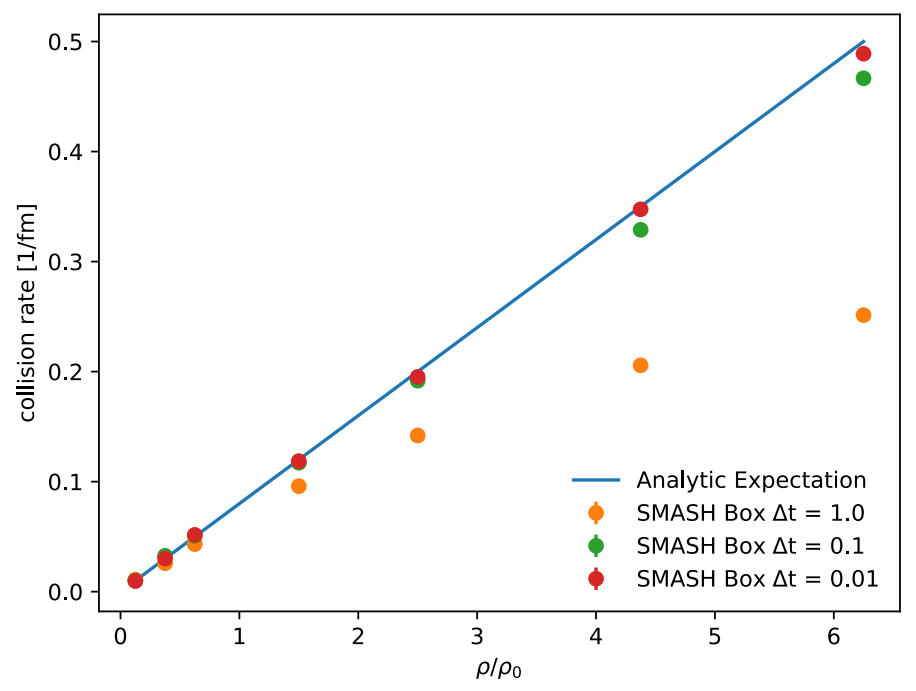

Figure 5.14: Collision rate in a box with $\pi^{0}$ mesons interacting with a constant elastic crosssection of $\sigma=10 \mathrm{mb}$ for different densities and time-step sizes compared with the analytic expectation of $n \sigma$. Density given in units of nuclear ground-state density $\left(\rho_{0}=0.16 \mathrm{fm}^{-3}\right)$. Calculation with the stochastic criterion.

$\Delta t$-sized timesteps. Note that for the geometric criterion, the evolution is often not done in timesteps, but the system is propagated from collision to collision (Section 2.2.1). This is not possible for the stochastic criterion since the collision probability is defined over an a priori known time-interval $\Delta t$.

The main limitation of a timestep-based evolution is given by the assumption that only one interaction per particle per timestep is possible. Therefore, the collision rate is underestimated if more than one collision per particle would be expected for the given timestep. This effect is illustrated by the result in Figure 5.14, which shows the collision rate for a $\pi^{0}$ gas in a box (see Section 2.3) at different densities. The analytic expectation (here simply $n \sigma$ ) for the collision rate is matched for the differently sized timesteps only for low-densities. The collision rate is underestimated for large densities if the timestep is (too) large since more than one collision per timestep would be necessary to match it. Therefore for all results produced with the stochastic criterion, the timestep needs to be small enough that secondary interactions within a timestep are avoided (see also results in Section 5.1.1). The drop in collision rate is not necessarily an effect of a rising density but is applicable to all cases, where more than one collision per timestep would become likely. A similar result, for example, is observed for an increase of the cross-section in Figure 5.1.

In terms of the collision probability, these results illustrate that the probability has to be small, such that numerically not more than one collision is accepted per timestep. Since the probability directly scales with the timestep $P \propto \Delta t$, a small $\Delta t$ ensures that this is the case. In addition, a small $\Delta t$ ensures that $P \leq 1$. Note that while $P \leq 1$ is necessary for $P$ to be used in a Monte-Carlo decision, it is not sufficient to avoid underestimating the collision rate. If the probability is large (while still $\leq 1$ ), it can still be likely that the Monte-Carlo decision accepts more than one interaction per particle per timestep. While this is theoretically a correct result, since the derived probability formula predicts it, all secondary interactions within a timestep are discarded in SMASH. In testing, a fraction of 
less than $1 \%$ of discarded interactions relative to the total number of interactions showed no effect on the scattering rate. Above this percentage, SMASH, therefore, emits a warning since the effect of a smaller timestep on the results has to be investigated specifically.

\section{Cell size}

Ideally, results do not depend on the chosen cell size since it is a free parameter of the approach. However, from the comment at the beginning of this section (about the need to choose an appropriate pair of $\Delta t$ and $\Delta^{3} x$ to limit the probability to 1 ), it is already clear that this is not the case from considering the probability equation alone. Therefore, the aim is to find a sensible interval for the cell size, in which results do not depend on the choice of $\Delta^{3} x$.

Studying the results for different cell sizes ${ }^{4}$ reveals that this interval is limited by two extreme cases, where the results start to exhibit a cell size dependence. On the one side, such a dependence appears for small cells if the cells are filled very sparsely. On the other side, the cells are too large when they overestimate the size of the medium itself. In the following, results illustrating both effects for small and large cells are shown and explained. In contrast to the effect of differently sized timesteps, the effect of the cell size is also different for reaction classes i.e. different numbers of incoming particles $n$, since the probability depends on $\frac{1}{\left(\Delta^{3} x\right)^{n-1}}$ (cf. Equation 5.13). So generally multi-particle reactions $(n>2)$ are more sensitive than 2-body reactions.

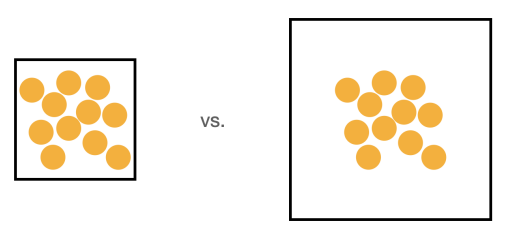

Figure 5.15: Illustration of cell-size overestimation. Black square displays the grid cell and yellow dots the particles inside the cell.

The cell size is too large if a single cell is large than the relevant medium itself. Consider the situation in Figure 5.15 as an example. Physically, the situation is identical in both scenarios, but the calculated scattering probability and with this the resulting scattering rate will be smaller on the right-hand side. Would the medium still be the same size or larger than the cell on the right-hand side, the smaller scattering probability would be compensated by the larger number of possible particle parings (note that in this case also more than one cell would be necessary on the left). The result would be a constant scattering rate. In other words, the cell size is overestimated if the medium inside each cell is highly inhomogeneous. The cells need to be small enough to resolve the particle distribution of the system.

Figure 5.16 illustrates how this overestimation is reflected in the total number of scatterings (= interactions with 2 or more particles incoming) in a sphere (see Section 2.3) of $\pi, N$ and $\bar{N}$ as a test setup $^{5}$ For this and the following results, the size of the grid cells is controlled

\footnotetext{
${ }^{4}$ How the cell sizes can be varied is detailed in Appendix A.2.

${ }^{5}$ Note that the grid for this result was modified to have a fixed size that is larger than the sphere to allow for cells to be larger than the sphere. This reflects the situation in a hadronic afterburner calculation,
} 


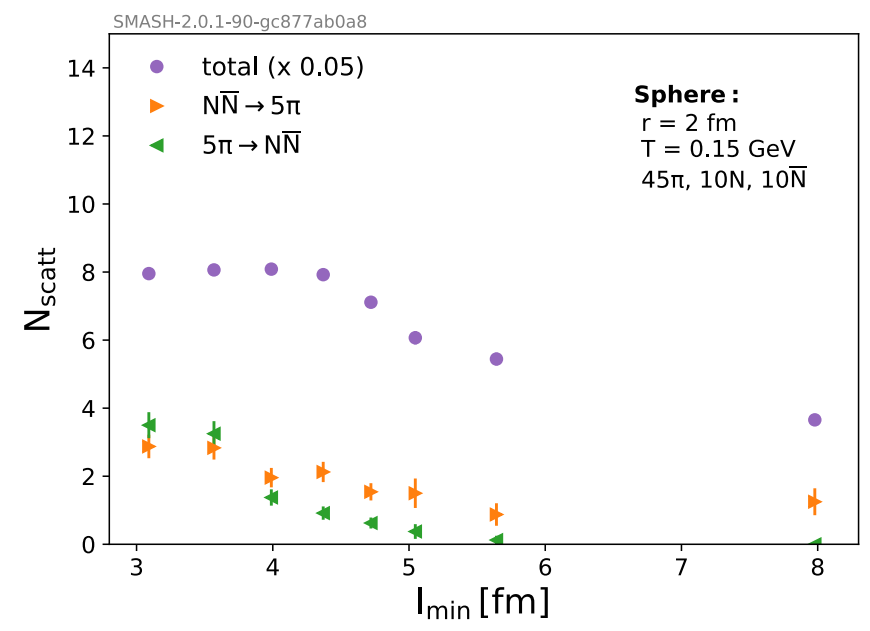

Figure 5.16: Number of scatterings in sphere of $\pi, N$ and $\bar{N}$ for different cell sizes (minimum cell lengths $\left.l_{\min }\right)$.

by giving a minimum value for the cell length $\left(l_{\min }\right)^{6}$. Only a minimal value is given since the equally-sized cells are scaled up to fill the whole grid size. Usually, the size of the cells is not significantly larger than the minimal value (especially if the number of cells is large). For the interpretation of the following results, this difference is also not relevant.

Figure 5.16 shows the (scaled down) total number of scatterings as well as the number of $N \bar{N} \rightarrow 5 \pi$ and $5 \pi \rightarrow N \bar{N}$ reactions. Clearly visible is the drop in the number of scatterings for larger cell sizes due to them being too large and the collision probability underestimated. However, it is also visible that below a certain cell size, the scattering number is constant and not dependent on the cell size as it should be. Furthermore, it is seen that the multi-particle reaction is more sensitive to the cell size and the number of $5 \pi \rightarrow N \bar{N}$ reactions start to decline already for smaller cells, also dragging the $N \bar{N} \rightarrow$ $5 \pi$ reaction down. This confirms that multi-particle reactions are more sensitive to the effect of a large cell size and has two reasons: As noted, the cell volume goes into the 5 -body reaction probability to the power 4 (and not 1 as for binary reactions). And, most $5 \pi \rightarrow N \bar{N}$ reactions happen early when the system is still dense and small and the cell size is too large for the spherical medium. In comparison, 2-body reactions are still happening later when the medium expands and the cell size is not overestimating the medium anymore. That the reaction number drops starting with (minimum) cell lengths of $l_{\min }=4 \mathrm{fm}$ is also clear when considering the size of the sphere $(r=2 \mathrm{fm})$. The cuboid gird cells overestimate the spherical medium when the diameter is matching the cell length. Note that the described effect for too large cells cannot be observed in a box since the grid is always as large as the box and, therefore, the homogenous medium cannot be overestimated.

While the overestimation effect is obvious when looking at the number or rate of reactions, how e.g. observables like the multiplicity are influenced might not be as trivial due to the

where the grid is much larger than the fireball due to the spectators at the grid borders. Normally, the grid size is determined by the minimum and maximum position of the particles, preventing an overestimation in the sphere setup.

${ }^{6}$ Technically, this is achieved by changing the maximum cross-section from the configuration file, as explained in Appendix A.2. 
different sensitivity of forward (multi-particle) compared to backward (2-body) reactions.
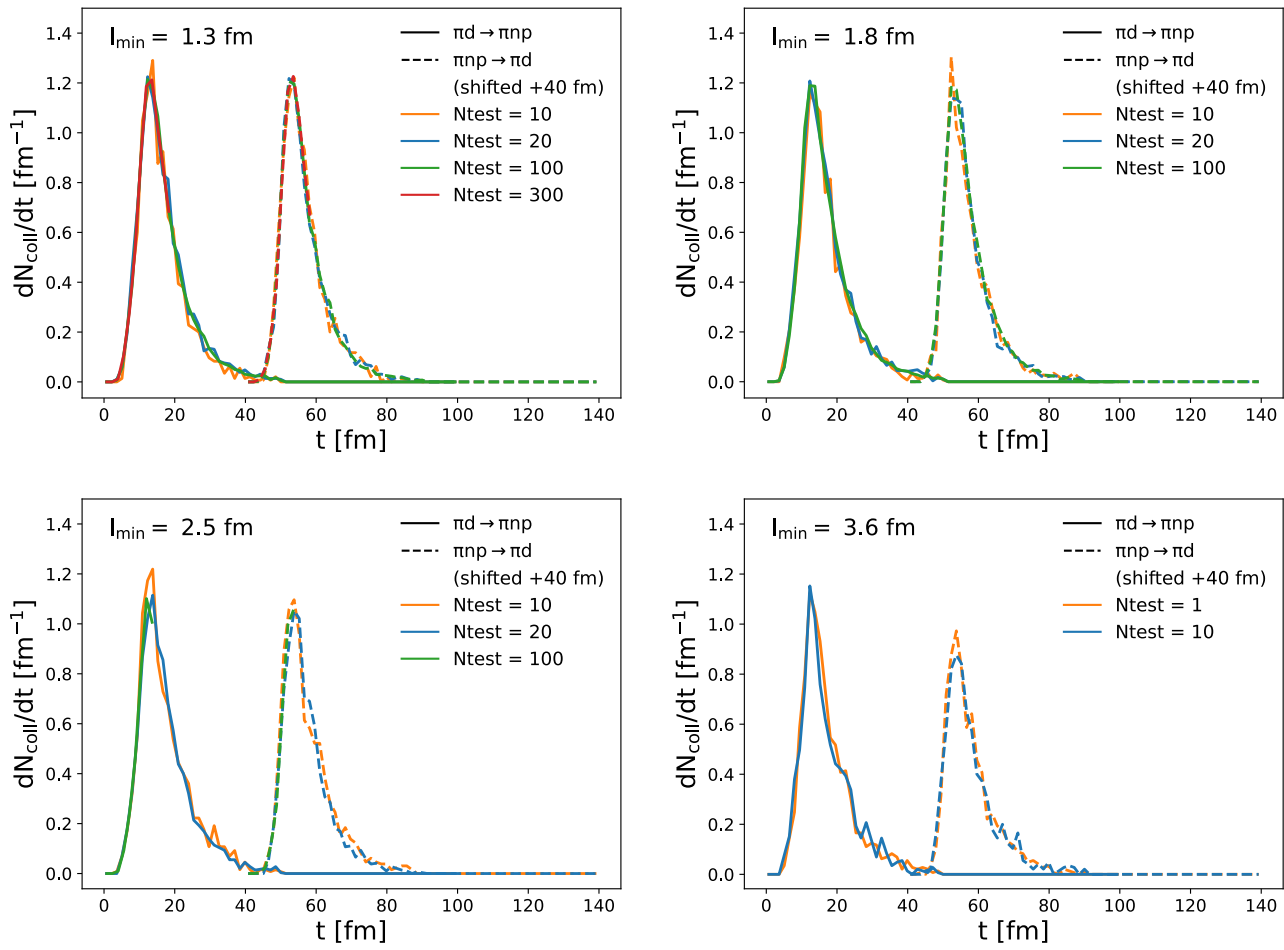

Figure 5.17: Collision rate of $\pi d \rightarrow \pi n p$ and $\pi n p \rightarrow \pi d$ in afterburner calculations for $\mathrm{AuAu}$ collisions at $\sqrt{s}=7.7 \mathrm{GeV}$ for different (minimum) cell sizes. The 3 -to-2 reaction rate (dashed lines) is shifted by $40 \mathrm{fm}$ for readability.

That the dependence for too large cell sizes is also observed in a more realistic scenario of an afterburner calculation for AuAu collision at $7.7 \mathrm{GeV}$ incident energy is seen in Figure 5.17. With the collision rate for the deuteron reactions remaining constant for the two upper plots with a $1_{\min }$ of $1.3 \mathrm{fm}$ and $1.8 \mathrm{fm}$, the rate starts to decline with a minimum cell length of $l_{\min }=2.5 \mathrm{fm}$, as seen for the more sensitive multi-particle reaction $\pi n p \rightarrow \pi d$. Nevertheless, also the $\pi d \rightarrow \pi n p$ two-reaction is affected. The change of the balance of forward and backward reactions in turn changes the multiplicity of the $d$ as seen in Figure 5.18. The overestimation of the cell size more severely affects the deuteron producing multi-particle reaction and, therefore, the yield is suppressed. Note that the afterburner results for deuteron reaction rate and multiplicity are obtained with a modified version of SMASH that blocks immediate back-reactions to prevent the below-described issue for too small cells.

To additionally visualize the overestimation of the cell size, Figure 5.19 shows the particle distribution of $d$, as the here relevant particle species, in the afterburner calculation at different times in the $\mathrm{x}$-z-plane. Also plotted is a cell equivalent to a cell length of $l_{\min }=2.5$ $\mathrm{fm}$, which is the first cell size that shows a decline in the collision rate in Figure 5.17. The figure illustrates that the overestimation in an afterburner calculation is along the beam (z) axis, where the nuclei are Lorentz-contracted. Comparing the medium size in the Lorentz-contracted nuclei region and the also shown example cell, it is plausible that such cells are too large and would therefore explain the declining number of (multi-particle) reactions and declining multiplicities for this size in the previous result. 


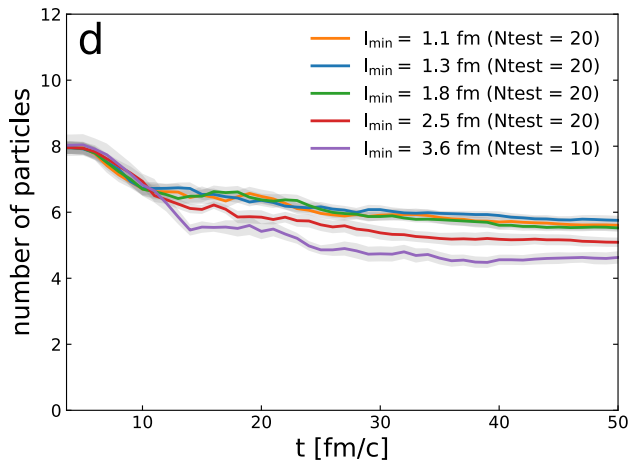

Figure 5.18: Multiplicity evolution of deuterons in afterburner calculations for $\mathrm{AuAu}$ collisions at $\sqrt{s}=7.7 \mathrm{GeV}$ for $\mathrm{AuAu}$ collisions at $\sqrt{s}=7.7 \mathrm{GeV}$ for different (minimum) cell sizes.
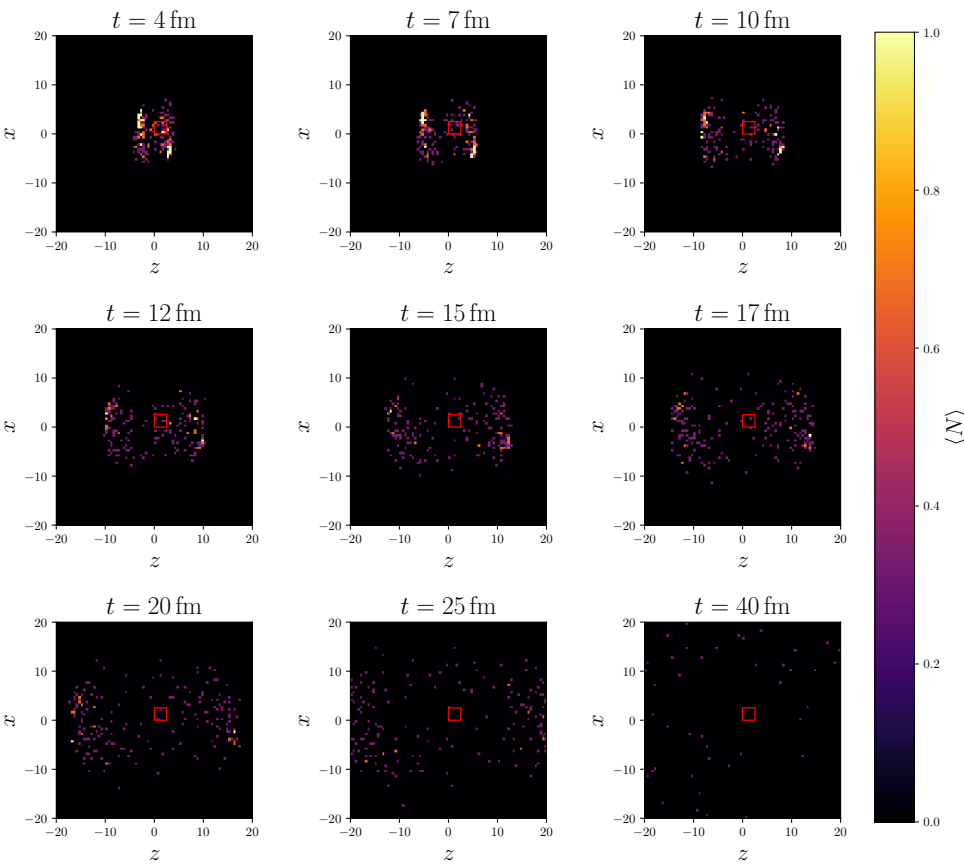

Figure 5.19: Distribution of deuterons in the x-z-plane in afterburner calculations for $\mathrm{AuAu}$ collisions at $\sqrt{s}=7.7 \mathrm{GeV}\left(N_{\text {test }}=20\right)$ including an example cell of size $l=2.5 \mathrm{fm}$ for scale. 
On the other hand, the cells can also be too small. Since only particles inside a cell are interacting, the scattering rate is expected to drop and stall eventually, when on average only one or fewer particles are inside a cell.

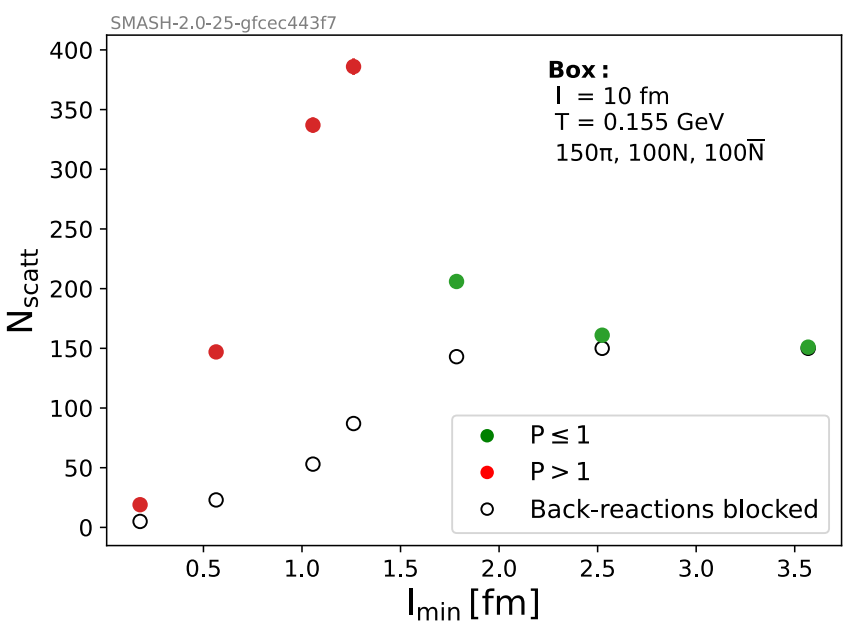

Figure 5.20: Number of scatterings for the first $30 \mathrm{fm}$ in a box calculation. Default calculation (filled symbols) and back-reactions artificially blocked (unfilled symbols).

Figure 5.20 shows the number of scattering for different cell sizes in a box. Surprisingly, even though the expected decline of scatterings is seen for tiny cells, the scattering number first rises when reducing the cell size. The calculations where the probability extends values of 1 are marked in red. Such calculations should normally be ignored and redone with adjusted simulation parameters. However, in this case, they are still interesting since the same issue of the rising scattering rate affects the $P \leq 1$ calculations (green markers), even though not as severe. In addition, the probability larger 1 , as mentioned in the discussion of the timestep size above, can be interpreted as the prediction of more than 1 scattering per particle. So, the expected effect of $P>1$ would be a decline of the scattering number, not a rise. Note that due to employing a box, the issue of overestimating the cell size is avoided.

The scattering rate is exploding for smaller cells because of immediate back-reactions of just performed scatterings. The outgoing particles are placed inside the cell of the incoming particles. If they do not propagate outside of the cell within the remaining timestep, a scattering of the previous outgoing particles might occur again since only outgoing particles of inelastic reactions are newly created and have to be placed. Conceptually, such back-reactions are valid and should be possible. But, with smaller cell sizes $\left(\Delta^{3} x\right)$, the probability is rising $\left(P \propto \frac{1}{\left(\Delta^{3} x\right)^{n-1}}\right)$ and if the cells are only filled sparsely, the back-reaction will at some point almost certainly happen, since there are no possible other processes between the particles inside the cell. The additional result displayed in Figure 5.20 proves that back-reactions explain the observed rising number of scatterings (empty black markers). That is, since the expected dropping dependence for small cell sizes is recovered if back-reactions are artificially blocked. As mentioned, even though back-reactions are a numerical artifact, conceptually, they should be possible. So, artificially blocking them is not a permanent solution but helps to illustrate the effect. The issue appears, after all, due to being in a parameter region, where the stochastic criterion is not expected to work: high probability (close to 1) and only few particles inside a cell. In particular, the latter 
makes a stochastic interpretation of the collision process difficult.
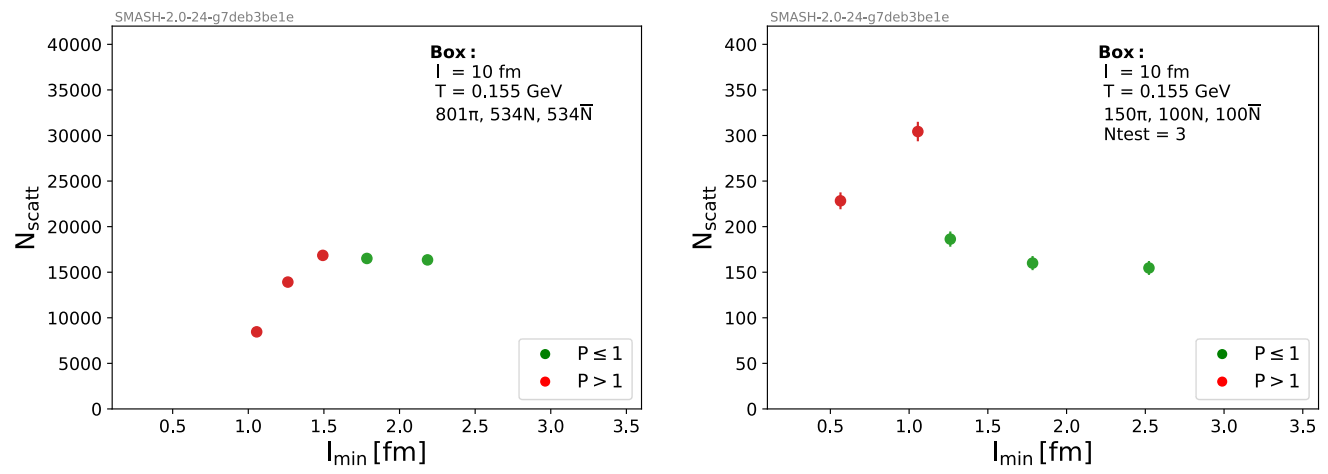

Figure 5.21: Number of scatterings for the first $30 \mathrm{fm}$ for different (minimum) cell sizes in a box calculation. Left: More particles. Right: More test particles.

In Figure 5.21 the number of scatterings is seen for more (test) particles inside the box. This resolves the factor of too few particles inside a cell or shifts the issue to even smaller cell sizes. Indeed, on the left, in a more dense system with more particles inside the box, the rise in the number of scattering vanishes. Only calculations with $P>1$ exhibit a change of scattering number. Of course, the density is a property of the physical system and cannot be changed outside test scenarios. Therefore, on the right plot, the more practical solution of employing test particles is shown. Here, the rising scattering rate is shifted since the smallest cell sizes still suffer from too few particles inside each cell. Still, the issue is resolved for cell sizes, where without test particles, the scattering number already starts to rise. The advantage of test particles is that also the probability is scaleddown and that they can be added as a calculation parameter at all times. As seen on the figure, the issue of $P>1$ is also shifted (cf. Figure 5.20).

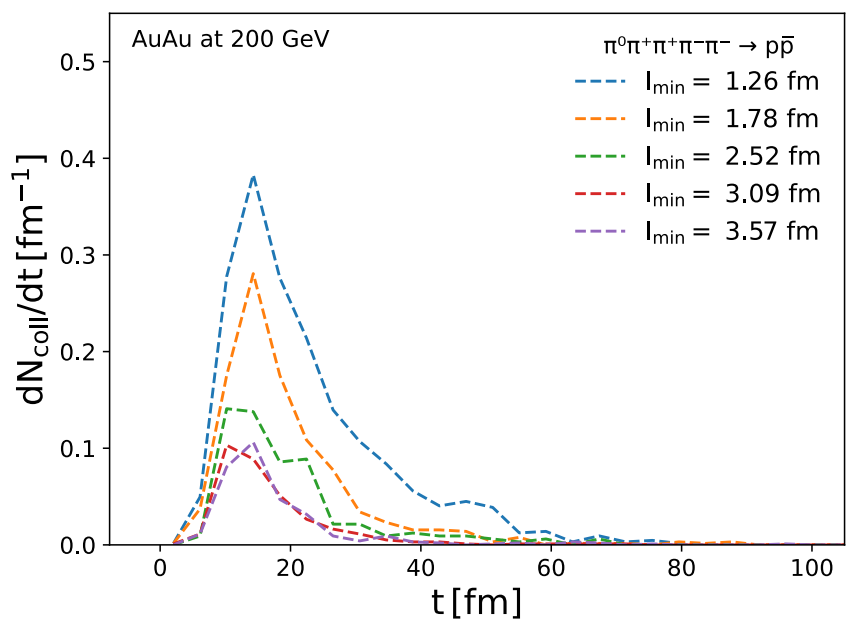

Figure 5.22: Collision rate of $5 \pi \rightarrow p \bar{p}$ in afterburner calculations for $\mathrm{AuAu}$ collisions at $\sqrt{s}=200 \mathrm{GeV}$ for different (minimum) cell sizes.

The issue of the cells being too small and a connected (counter-intuitive) rise of the scattering number can, of course, also be avoided by employing large enough cells. Figure 5.22 shows the scattering rate of $5 \pi \rightarrow p \bar{p}$ in an afterburner calculation for $\mathrm{AuAu}$ collision at 
a beam energy of $200 \mathrm{GeV}$. Also, here, in this physically more realistic calculation, the rise of the scattering rate is observed for small cells, with the 5-body scattering rate being particularly sensitive. But for the two largest (minimum) cell lengths, the scattering saturates. As long as the issues for small and large cells do not overlap, which is the case for the large medium produced at the high beam energies, it is sufficient just to employ cells of these sizes.

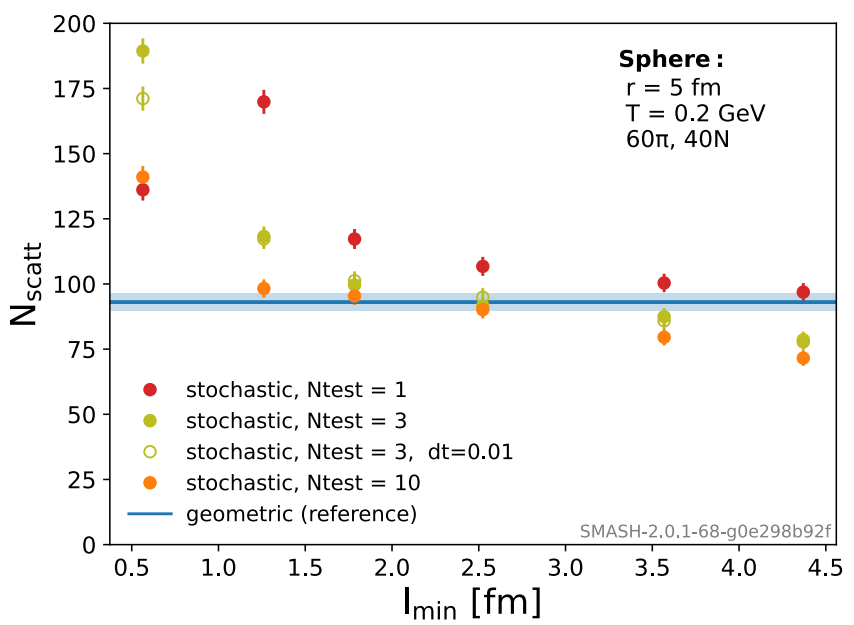

Figure 5.23: Number of scatterings for different (minimum) cell sizes with the stochastic criterion in an expanding sphere scenario. Reference calculation with the geometric criterion done for default $l_{\min }$ (corresponding to default of $\sigma_{\max }=200 \mathrm{mb}$ ) and $N_{\text {test }}=1$.

Figure 5.23 illustrates both observed cell size dependencies in a sphere and thereby summarizes the discussion of the cell size. The rise and drop of scatterings are seen for small and large cells, respectively. In case of $N_{\text {test }}=1$ both effects are even overlapping. Since the issue for small cells is improved upon with test particles, the lessons ${ }^{7}$ to avoid a cell size dependence could be summarized as follows: The cells should be small, but at the same time situations, where back-reaction become artificially enhanced need to be avoided. Therefore, the cells in the medium need to be sufficiently filled and the calculated probabilities should be small. Both are achieved by employing (more) test particles ${ }^{8}$.

\footnotetext{
${ }^{7}$ with the current implementation of the grid

${ }^{8}$ The number of necessary test particles is depending on the concrete scenario and has to be explored on a case by case basis.
} 


\section{Summary}

The extensive verification provided in this section establishes the new framework for stochastic rates. It justifies their application for the microscopic dynamics of heavy-ion collisions for the remainder of this chapter and future studies. The results obtained with the stochastic criterion agree perfectly with the available analytic calculations for all cases studied, in contrast to the geometric criterion. They mark the first demonstration of detailed balance for multi-particle reactions in SMASH. 5-body reactions are described for the first time in any transport approach. The numerical stability under the variation of different calculation parameters is shown and its limits are determined.

Furthermore, it is observed that the system equilibrates faster when employing multiparticle reactions in comparison to an equivalent binary reaction treatment. This observation is made consistently for all described multi-particle reactions (3-to-1, 3-to-2 and 5 -to-2). The effect of this faster equilibration in realistic systems on experimental observables is studied in the following. 


\subsection{Three-body decay back-reactions in nucleus-nucleus col- lision}

The introduction of the 3-to-1 (back-) reactions $\pi \pi \pi \rightarrow \omega, \pi \pi \pi \rightarrow \phi$ and $\pi \pi \eta \rightarrow \eta^{\prime}$ (Section 4.3.1) enables the study the respective Dalitz decays of $\omega, \phi$ and $\eta^{\prime}$, while fulfilling detailed balance. The presented calculations in this section are the first multi-particle reaction results in realistic heavy-ion collisions as they are measured by experiments. They are based on the verification presented in the previous Section 5.1. The beam energies studied are lower as e.g. accessible by HADES or the upcoming CBM experiment. While the 3-to-2 deuteron and 5-to-2 $p \bar{p}$ reaction address the more topical research questions, the results for 3-to- 1 complement the multi-particle studies in this work and provide first insights into their relevance. While it is found that their relevance for the medium overall is minimal, the following results nevertheless quantify the number of reactions and are a helpful first test case in a realistic hadronic medium. In particular, because of the similarity of the different approaches for $d$ or $p \bar{p}$ reactions and the $\omega$ Dalitz decay. The multi-particle reaction in both cases is either treated directly (via the introduced stochastic criterion) or via a two-body reaction chain (also possible with the geometric criterion).

The collision system for all results (except for the system size investigation) is AuAu. The collisions are always central $(b=0)$. The idea is to maximize the density of the medium and thereby the effect of multi-particle reactions. The results, therefore, are an upper limit of the 3 -to- 1 scattering numbers.
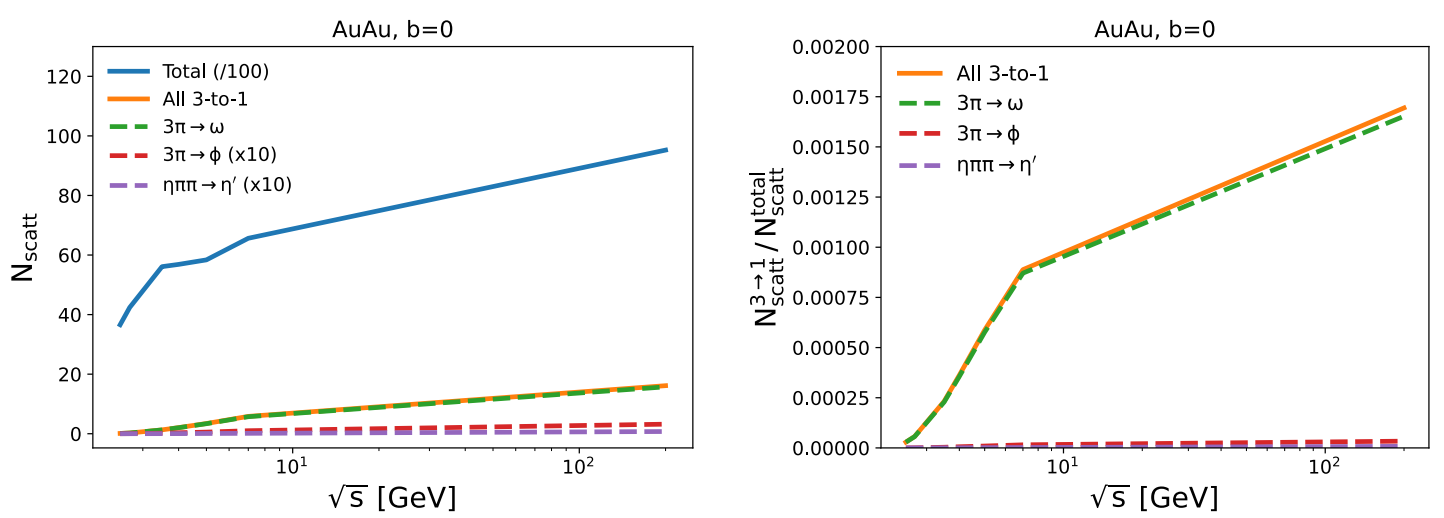

Figure 5.24: Number and ratio of 3-to-1 scatterings compared to the total number of scatterings (per event) in AuAu collisions for different beam energies (impact parameter $b=0 \mathrm{fm}$ ). Some scattering numbers scaled down (up) for readability.

First, the energy dependence of the total number of 3-to-1 scatterings in AuAu events is investigated. Figure 5.24 reveals that the number of 3 -to- 1 reactions is around 5 orders of magnitude smaller than the total number of scatterings. Here the total includes all reactions, also decays. 3-to-1 reactions for mesonic resonances are therefore irrelevant for the overall bulk dynamic and evolution of the system. An effect will only be observed for specific observables.

However, if one compares the number of 3-to-1 reactions to the number of the reverse Dalitz decays, a sizable regeneration of the resonances by the multi-particle back-reactions is seen (Figure 5.25). A ratio of around 0.25 is found with a slight decline towards higher energies. This means, on average, every fourth decay has a back-reaction. 

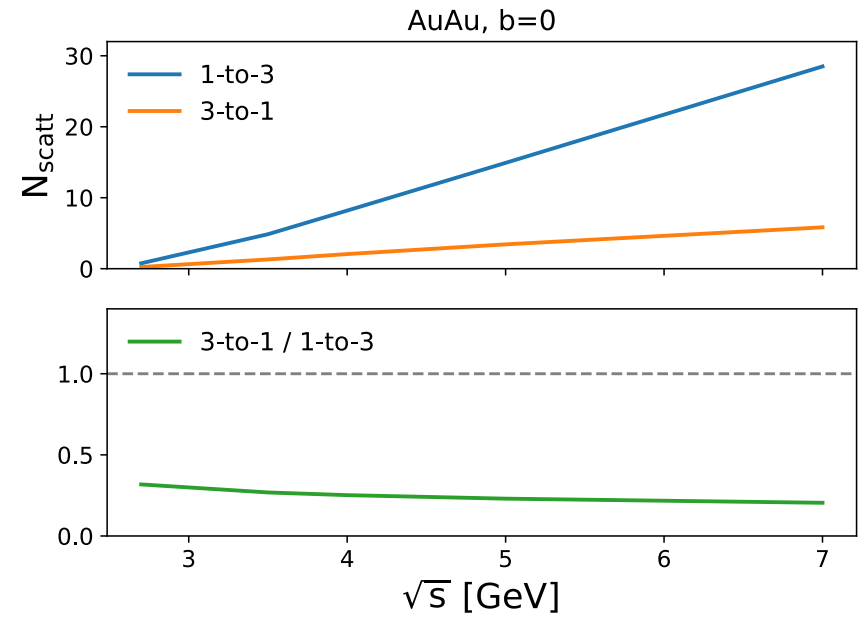

Figure 5.25: Number and ratio of 3-to-1 scatterings (per event) and 1-to-3 decays in AuAu collisions for different beam energies (impact parameter $b=0 \mathrm{fm}$ ).

The two approaches for fulfilling detailed balance for the $\omega$ Dalitz (direct $\omega \leftrightarrow \pi \pi \pi$ or $\rho \leftrightarrow \rho \pi \leftrightarrow \pi \pi \pi$ ) are found to be overall comparable, similar as for the reported for box calculations in Section 5.1.2. The ratio for $3 \pi \rightarrow \omega$ over $\rho \pi \rightarrow \omega$ found in testing at low beam energies is between $0.8\left(\mathrm{AgAg}\right.$ at $\left.E_{\mathrm{Kin}}=1.58 \mathrm{GeV}\right)$ and $0.85(\mathrm{AuAu}$ at $\left.E_{\text {Kin }}=1.23 \mathrm{GeV}\right)$ i.e. the 3-particle reaction appears to be (15-20\%) less likely. Note, however, that the reported numbers are upper limits for the difference since the $\pi \rho$ pair reacting to form a $\omega$ meson does not necessarily have to be produced by $3 \pi$. Therefore, other processes might feed the counted $\rho \pi \rightarrow \omega$ reactions. Two results discussed in this work would be in principle sensitive to a change in $\omega$ number caused by a difference in the 3-to-1 back-reaction treatment: the $\omega$ pp cross-section (Figure 3.3) and $\omega$ pole mass region of the different dielectron invariant mass spectra presented in Section 3.1. Unfortunately, neither of the results is experimentally constrained precise enough to differentiate an effect of the order of $20 \%$.
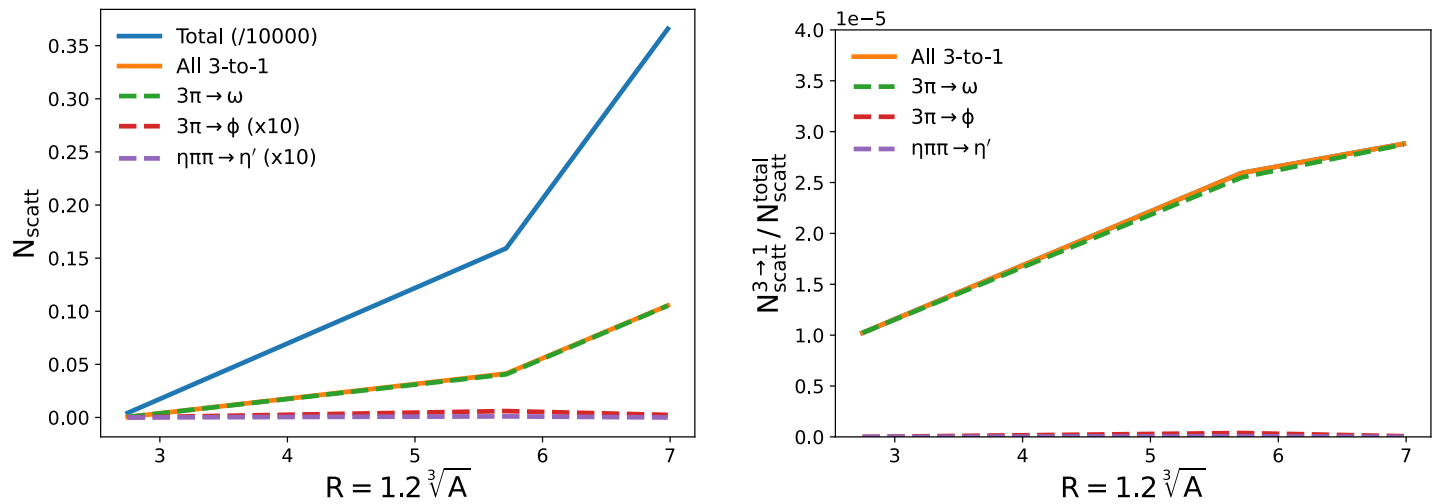

Figure 5.26: Number and ratio of 3-to-1 compared to the total number of scatterings (per event) for different system sizes at a kinetic beam energy of $1.5 A \mathrm{GeV}$ and impact parameter $b=0 \mathrm{fm}$. System size characterized by radius of nucleus given by $R=1.2 \sqrt[3]{A}$, where $A$ is the number of nucleons in the nuclei. Studied systems: CC, AgAg, AuAu. Some scattering numbers scaled down (up) for readability. 
In Figure 5.26 the effect of the system size on the 3-to- 1 reaction number is shown. Three systems $^{9}$ are probed, $\mathrm{CC}, \mathrm{AgAg}$ and $\mathrm{AuAu}$, all at a kinetic beam energy of $1.5 \mathrm{~A} \mathrm{GeV}$ and impact parameter $b=0 \mathrm{fm}$. As a proxy for the system size, the radius of a nucleus is calculated with $R=1.2 \sqrt[3]{A}$ ( $A$ is the number of nucleons in the nuclei). As expected, the number of 3-to-1 increases with the system size since the density of the created medium is higher. The same is also true relative to the number of total scatterings, where the ratio triples from $\mathrm{CC}$ to $\mathrm{AuAu}$, even though it remains small in absolute terms.

Comparing the different 3-to-1 reactions in Figure 5.26 as well as Figure 5.24, it appears that only the $3 \pi \rightarrow \omega$ reaction is of relevance and essentially equals the total 3 -to- 1 reactions. The reason is a combination of kinematics and decay width of the resonance states, which suppress the $\phi$ and $\eta^{\prime} 3$-to- 1 channel compared to $\omega$. All three, $\omega, \phi$ and $\eta^{\prime}$, resonances are narrow i.e. have relatively small decay widths, which already suppressed the back-reaction in general. But, the $\eta^{\prime}$ width is another order of magnitude smaller than the one of $\omega$ and $\phi$. In addition, the mass of $\phi$ is $30 \%$ larger; its width is half the $\omega$ meson width, and the Dalitz $3 \pi$ decay only has a branching ratio of $20 \%$ for $\phi$ (in comparison to $89 \%$ for the $\omega)$.
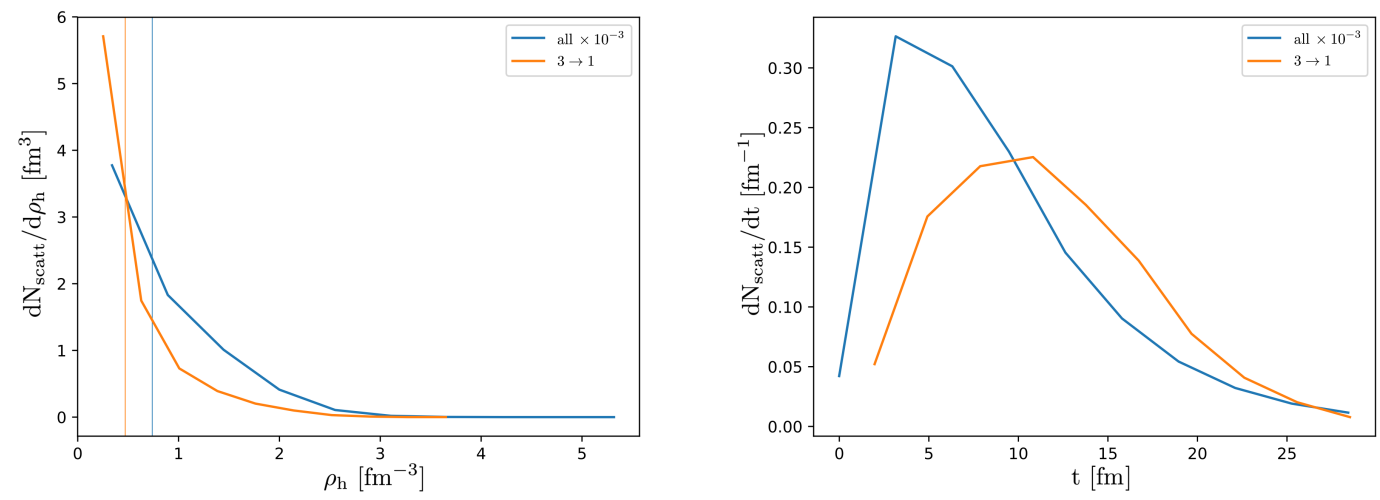

Figure 5.27: The number of 3-to-1 and total scatterings for different hadron densities (left) and times (right) in AuAu collision at $\sqrt{s_{N N}}=5.0 \mathrm{GeV}$. Vertical lines in the left panel are the mean densities. Total number scaled down for readability.

Complementing the results for the number of scatterings are first calculations that show the scattering numbers differentially in density and time in Figure 5.27. Interestingly, the (mean) density at which 3-to-1 reactions are happening is observed to be lower than for all reactions, which is opposite to a first expectation. Since more particles are necessary, one would expect that the density, on average, needs to be higher for multi-particle reactions. The right plot in Figure 5.27 for the scattering rate partly explains this contradiction. The 3 -to- 1 reaction rate peaks later in time than the total reaction rate. The total reaction here may be dominated by the initial scatterings and subsequent decay chains, which would explain an earlier peak. Or, the 3-to- 1 reaction rate is potentially delayed because the necessary pion abundance first needs to be produced. The different processes that contribute to the total reaction rate need to be differentiated to fully understand this effect, which is left for future work. In any case, the shifted 3-body reaction rate explains the difference in (average) density since, in general, as the system expands, also the density drops over time.

\footnotetext{
${ }^{9}$ Note that since the stochastic criterion (in SMASH) is not applicable, no elementary systems are included.
} 
Overall, the results for 3-to- 1 reactions in this section are a constructive first step to explore multi-particle reactions in heavy-ion reactions. 3-to-1 reactions are not relevant for the overall dynamic of the system, with their reaction rate being sub-leading in comparison to the total scattering rate. Therefore, to observe an effect, sensitive observables for $\omega, \phi$ and $\eta^{\prime}$ need to be considered to resolve that $25 \%$ of all studied Dalitz decays have a backward reaction. The most promising resonances being $\omega$, which back-reaction dominates the 3 -to-1 reaction rate. 


\subsection{Deuteron production in afterburner calculations}

This section is based on [4].

In this section, the dynamic deuteron production employing the microscopic three-body deuteron catalysis reactions is explored on the example of AuAu collisions at $\sqrt{s_{\mathrm{NN}}}=7.7$ $\mathrm{GeV}$. The reactions include $\pi p n \leftrightarrow \pi d, N p n \leftrightarrow N d$ and $\pi d \leftrightarrow N N$. The most important of these reactions is the pion catalysis $(\pi p n \leftrightarrow \pi d)$, since at energies above $\sqrt{s_{\mathrm{NN}}} \approx 5$ $\mathrm{GeV}$, it starts to dominate, as illustrated by the following results. At lower collision energies, baryons dominate at mid-rapidity; therefore, nucleons are the most frequent catalysts [126]. The $\pi d \leftrightarrow N N$ reaction is sub-leading in all cases due to its smaller cross-section.

Deuteron observables in heavy-ion collisions are usually not computed by a dynamical approach, but, as explained in Section 1.3.3, either by final-state coalescence from nucleons or by a thermal approach assuming chemical equilibrium of deuterons with hadrons. Such approaches neglect any $d$ reaction dynamic. Both model types reproduce experimental data with radically different assumptions about the deuteron formation ("snowballs in hell" paradox). Thermal models assume early and coalescence models late formation of deuterons. The dynamical transport approach employed here allows contrasting both deuteron production pictures by probing the deuteron evolution in the late (afterburner) stages of the AuAu collisions. For this, two scenarios at particlization ${ }^{10}$ are distinguished. In one case, deuterons are assumed to be produced in the hydrodynamic stage of the collision ( $w / d$ at particlization); in the other case, no deuterons are present at the start of the afterburner calculation ( $w / o$ d at particlization). The distinction allows to compare the two different pictures for deuteron production: the thermal model-like picture, where $d$ are produced early at high temperatures and the coalescence-like picture, where $d$ are assumed to be formed at later times from nucleons close in phase space.

Studying the microscopic deuteron production by pion catalysis is an idea introduced in [183] for central $\mathrm{Pb}+\mathrm{Pb}$ collisions at LHC energies and further tested for non-central collisions and at lower energies down to $\sqrt{s_{\mathrm{NN}}}=7.7 \mathrm{GeV}[135,184]$. However, these earlier works received criticism [306], because the approach for the $\pi p n \leftrightarrow \pi d$ reaction involves the non-existent intermediate resonance $d^{\prime}$ (Section 2.1.2). The results in this section build on those studies and reassess their conclusions on a more solid theoretical basis with the new (direct) multi-particle reactions. They also allow to compare both approaches for deuteron catalysis reactions and estimate the effect of the multi-step 2-to-2 $d^{\prime}$ treatment on the results.

Note that the validation of the equilibrium properties for both treatments presented in Section 5.1.2, i.e. exhibiting the correct chemical equilibration and detailed balance, is essential for the following investigations of AuAu collisions. The two treatments are verified by the agreement with the analytic rate equation solution in a box, as seen in Figure 5.9, and the more general validation of the stochastic criterion discussed throughout Section 5.1. The box results for the deuteron reactions in Figure 5.9 already reveal (in accordance with the rate equations) a faster equilibration for the direct 3 -to- 2 reactions compared to the 2-to-2 approach - a finding also observed for the 3-to- 1 and 5-to- 2 multi-particle reactions (cf. Figure 5.8 and 5.10). This leads to the interesting question of whether and how this

\footnotetext{
${ }^{10}$ Particlization means the switching from the hydrodynamical approach to the transport approach by (Cooper-Frye) sampling the different hadron species on the hydro hyper-surface. The sampled hadrons provide the initial conditions for the transport approach.
} 
difference manifests itself in a collider (afterburner) setup.

A hybrid approach is employed as the state-of-the-art for central $\mathrm{AuAu}$ collisions at $\sqrt{s_{\mathrm{NN}}}=7.7 \mathrm{GeV}$. All presented results only display the deuteron evolution in the afterburner stage of the hybrid approach. The hydro approach is MUSIC v3.0 [92, 93, 307, 308] with a smooth parametrized initial energy density [309] that is tuned to reproduce charged particle spectra and yields. As the equation of state, the lattice QCD based "NEOSBSQ" [310] is chosen. Shear viscous corrections are included, while bulk viscous corrections and baryon number diffusion are neglected. Particlization is performed at a constant energy-density hypersurface, $\epsilon\left(\tau, x, y, \eta_{s}\right)=0.26 \mathrm{GeV} / \mathrm{fm}^{3}$. This condition corresponds to almost the same temperature but slightly lower energy density and baryon chemical potential as the thermal model fit of the hadron yield at chemical freeze-out for the same collision system [311]. The particlization is performed with a standard grand-canonical Cooper-Frye procedure including shear viscous corrections [92, 93]. As mentioned, whether deuterons are sampled depends on the calculation. The $d^{\prime}$ resonance is never sampled. Most importantly, the hydrodynamic calculation produces a sensible distribution of pions and nucleons as an initial condition for the afterburner. As shown in [184], the experimental rapidity or transverse mass spectra of $\pi$ and $N$ are well reproduced by the provided distributions. On this basis, the $d$ production employing the two catalysis reactions is studied.

\section{Results}

All following results are for the deuteron production in the late stages of $\mathrm{AuAu}$ collisions at $\sqrt{s_{\mathrm{NN}}}=7.7 \mathrm{GeV}$ employing the mentioned catalysis reaction, $\pi d \leftrightarrow N N$ and elastic collisions between $d$ and $N$ or $\pi$. The 3-to-2 reactions are either direct multi-particle reactions (3-to-2 treatment) or they are modeled with a 2 -to-2 reaction chain including the $d^{\prime}$ resonance (2-to-2 treatment). The latter approach is either done with the geometric (geometric 2-to-2) or the stochastic (stochastic 2-to-2) criterion to also probe differences stemming from the criterion itself.

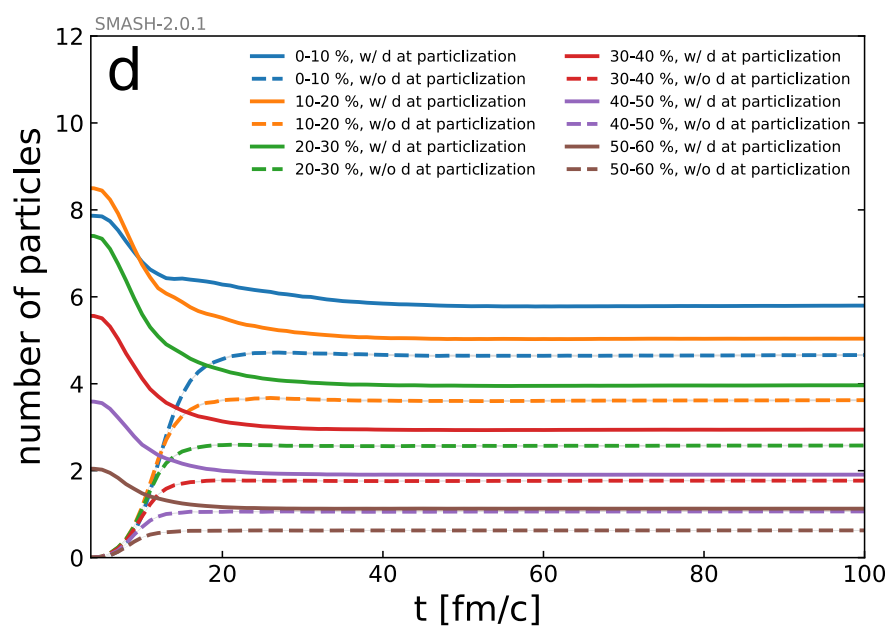

Figure 5.28: Evolution of deuteron yields in AuAu afterburner stage for all centrality class (3-to-2 approach).

Figure 5.28 shows the number of all deuterons propagated in the afterburner stage over time for all considered multiplicity classes from central $0-10 \%$ up to peripheral $50-$ 
$60 \%$ when employing direct stochastic 3-to-2 reactions. Apart from the initial $d$ number calculated from the hydro hyper-surface at $0-10 \%$ being initially lower than $10-20 \%$, the multiplicities are clearly and consistently ordered by centrality for both particlization cases.
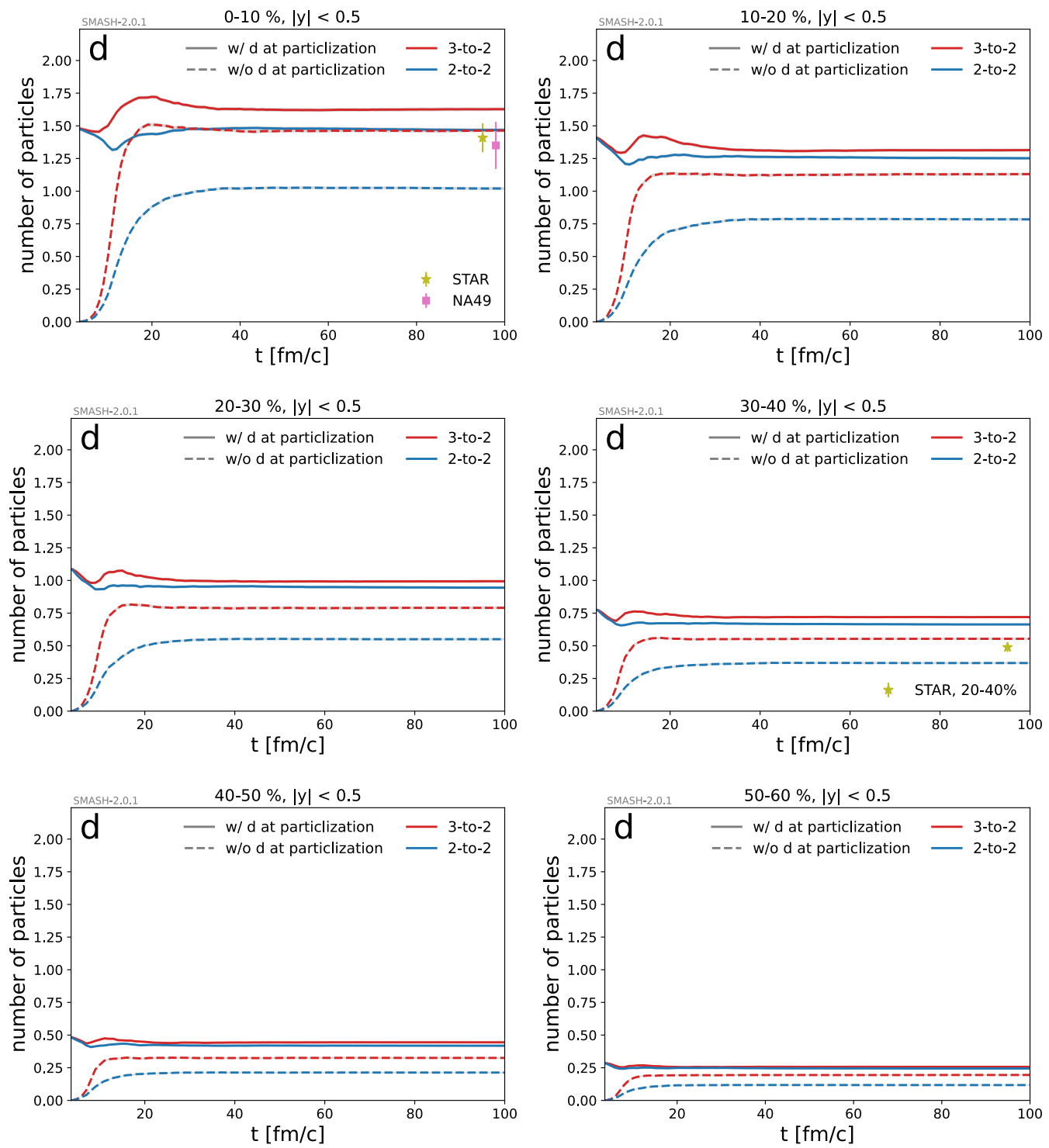

Figure 5.29: Evolution of mid-rapidity deuteron yields in AuAu afterburner stage for different centrality classes. Experimental data from [312, 313].

In Figure 5.29 the different approaches (3-to-2 and 2-to-2) are compared at mid-rapidity as the most interesting, since experimental accessible, rapidity region. The $d$ production is enhanced when employing direct 3-to-2 reactions. Especially in the case with no $d$ at particlization, a more rapid increase of the $d$ number is observed, which drives the number close to the case with $d$ at particlization. The final number of deuterons is almost identical for the two particlization scenarios. The remaining difference is on the order of experimental errors.

This result sheds light on the apparent contradiction of thermal and coalescence models and why both match experimental data. The $d$ yield stays approximately constant if $d$ are 


\begin{tabular}{c|c} 
Centrality & $\mathrm{N}_{\mathrm{d}, \text { equil }}^{\mathrm{w} / \mathrm{o}} / \mathrm{N}_{\mathrm{d}, \text { equil }}^{\mathrm{w} /}$ \\
\hline $0-10 \%$ & 0.900 \\
$10-20 \%$ & 0.860 \\
$20-30 \%$ & 0.795 \\
$30-40 \%$ & 0.769 \\
$40-50 \%$ & 0.731 \\
$50-60 \%$ & 0.759
\end{tabular}

Table 5.5: Ratio of the equilibrated $d$ yields for the two particlization scenarios.

initialized at the beginning of the afterburner (thermal model picture). The same yield is also generated with a later production of $d$ from nucleons (coalescence model picture) with no initial $d$.

The difference between yields is smaller for the calculation with the 3 -to- 2 reactions in comparison to the 2-to-2 approach. Those findings are understandable considering the above observed faster equilibration when employing multi-particle reactions. The 3 -to- 2 reactions drive the system faster to statistical equilibrium before it freezes out due to its expansion. The even smaller difference with 3 -to- 2 reactions subsequently validates and confirms the conclusions drawn in [135] with 2-to-2 reactions. The expansion is also why the yields without $d$ at particlization are not in full agreement with $d$ at particlization. The $\mathrm{d}$ reactions seize too quickly due to the cooling before enough $d$ can be produced (cf. Figure 5.31 and 5.32). Both particlization scenarios also agree with the experimental values for $0-10 \%$ centrality, which shows that it is possible to reproduce the $d$ yield with the assumption of multi-particle catalysis reaction as the main production mechanism. Comparing the presented centrality classes in Figure 5.29, the more peripheral collisions naturally produce less $d$ in general. The two deuteron reaction treatments also produce more similar yields in the case with $d$ at particlization.

Going from central to more peripheral collisions, the equilibrated yield without $d$ at particlization $\left(\mathrm{N}_{\mathrm{d}}^{\mathrm{w} / \mathrm{o}}\right.$ equil $)$ in comparison to the final yield with $d$ at particlization $\left(\mathrm{N}_{\mathrm{d}}^{\mathrm{w} / \text { equil }}\right)$ is less in agreement as Table 5.5 shows. The smaller medium created in peripheral collisions seems to suppress the full statistical equilibration of the system before freeze-out when all $d$ are produced in the late (afterburner) stages.
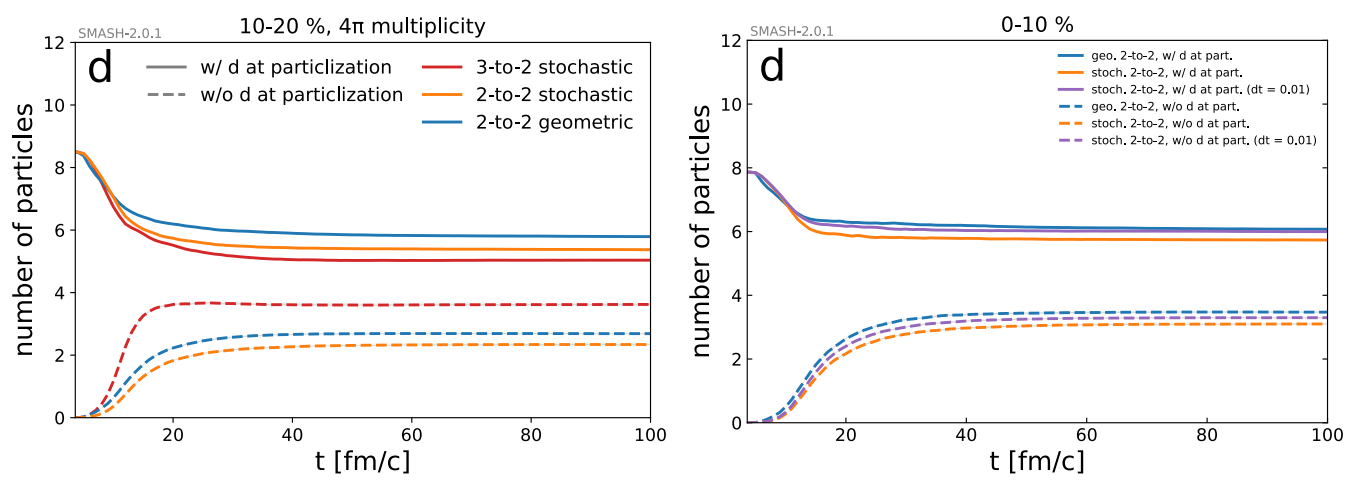

Figure 5.30: Evolution of $3 \leftrightarrow 2$ reaction rates in AuAu afterburner with d at particlization. Left: $0-10 \%$ centrality class. Right: $4 \pi$ multiplicity for $0-10 \%$ including stochastic 2 -to-2 reactions with smaller $\Delta t=0.01 \mathrm{fm}$. 
The yield comparison for all three approaches for the catalysis reactions is shown for the highest initial $d(4 \pi)$ multiplicity (10-20\% centrality) in Figure 5.30. Most importantly, the results illustrate that employing the stochastic criterion for the 2 -to- 2 reaction chain yields similar results as reported above, obtained with the geometric criterion. The small remaining difference between the two 2-to-2 treatments is minimized when the timestep is chosen smaller, as seen on the right plot in Figure 5.30. The number of test particles has been chosen to be $N_{\text {test }}=20$ for all results. It was separately verified that higher numbers of test particles and different cell sizes match the displayed results. The timestep for the below reported stochastic 2-to-2 results is chosen sufficiently small in the following as a consequence of the finding in Figure 5.30.
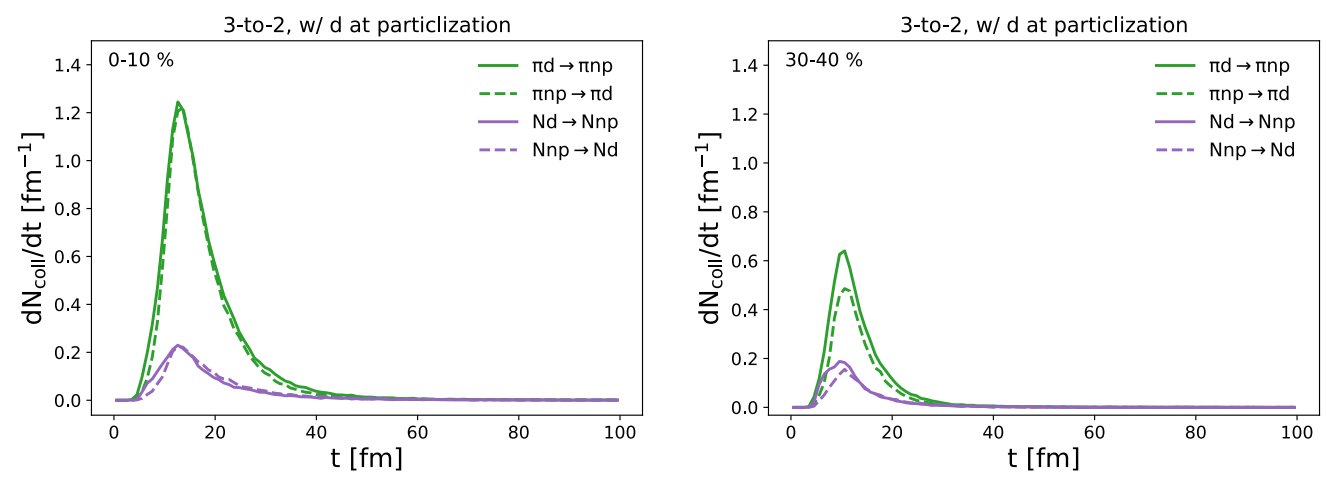

Figure 5.31: Evolution of $3 \leftrightarrow 2$ reaction rates in AuAu afterburner with d at particlization. Left: $0-10 \%$ centrality class. Right: $30-40 \%$ centrality class.

The evolution of the $d$ yield is the result of the competing $3 \rightarrow 2$ formation and $2 \rightarrow 3$ break-up reaction rates shown in Figure 5.31 for the case with $d$ at particlization and in Figure 5.32 for without $d$ at particlization. Forward and backward (direct) 3-to-2 reactions rates are close for central collisions in Figure 5.31, but as also seen for the yield, some time is necessary before they are close to being equilibrated. The catalysis reactions proceed rapidly enough to keep deuterons in relative equilibrium with nucleons. This explains the "snowballs in hell" paradox i.e. the light nuclei do not survive. Instead, they are destroyed and created at similar rates, keeping the overall yield constant.

The collision rates also clearly indicate the dominance of the $\pi$ catalysis reactions. This underlines the necessity to include the $\pi$ in addition to the $N$ reactions for this beam energy, which is the main extension compared to [126]. The $\pi$ reactions are also closer to being equilibrated than the $N$ reactions. For more peripheral reactions and hence a smaller medium, the $2 \rightarrow 3$ rate dominates over the formation reactions. This relatively lower $3-$ to-2 reaction rate again hints at incomplete statistical equilibration in the smaller system. Considering the calculation without $d$ in Figure 5.32, the $3 \rightarrow 2$ reaction dominates for all rates, as expected by the rapid rise of $d$ numbers at the beginning of the evolution. The reaction rate figures also allow to pinpoint a chemical freeze-out at least for the shown $3 \leftrightarrow 2 \mathrm{~d}$ reactions at around $50 \mathrm{fm}$ for central collisions (0-10\%). Since the system is smaller for more peripheral collisions (30-40\%), the freeze-out is earlier at around $30 \mathrm{fm}$. Note that while the $N N \leftrightarrow \pi d$ reaction is also included in the calculation, its contribution is only sub-leading, even compared to the $N$ catalysis reaction, and therefore the rate is not shown.

In addition to the yields, first, the average transverse momentum is presented for protons 

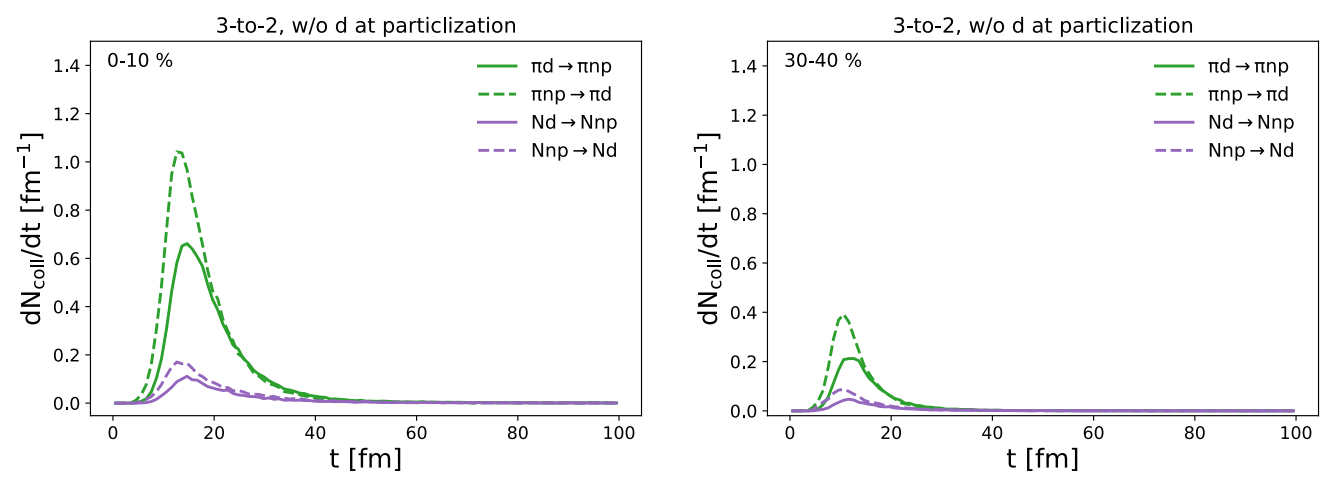

Figure 5.32: Evolution of $3 \leftrightarrow 2$ reaction rates in $\mathrm{AuAu}$ afterburner without d at particlization. left: $0-10 \%$ centrality class. Right: $30-40 \%$ centrality class.
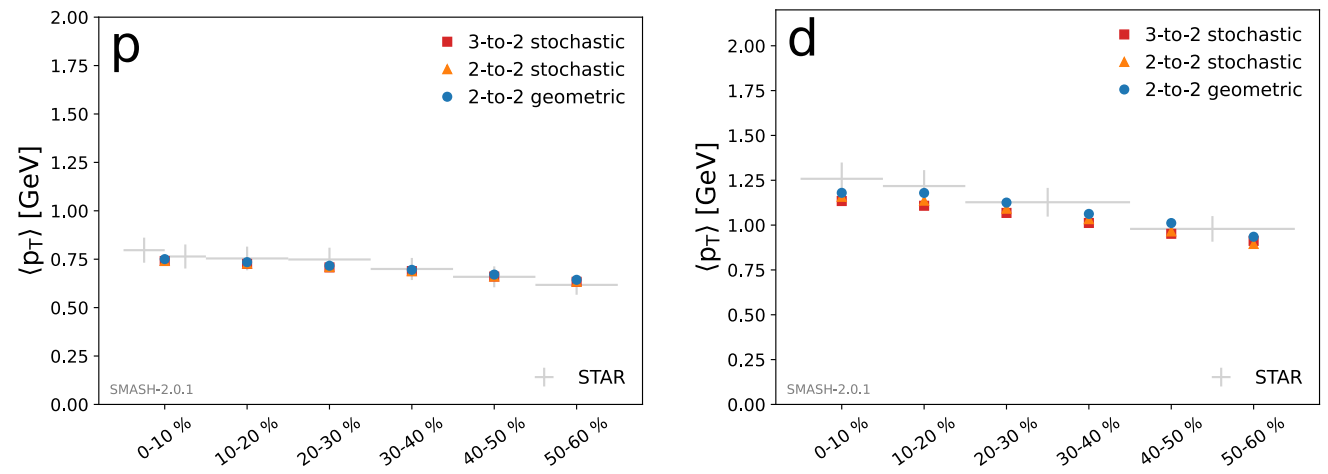

Figure 5.33: Average transverse momentum for protons (top) and deuterons (bottom). Experimental data from $[311,314]$.

and deuterons in Figure 5.33 for six centrality classes. The mean- $p_{T}$ slightly declines towards more peripheral collisions. Here and in the following, three treatments for the deuteron catalysis reactions are compared: calculating with the 2-to-2 reaction chain for the geometric (blue round points) and the stochastic (orange triangles) criterion as well as direct 3-to-2 reactions (red squares) that are only possible with the stochastic criterion. The mean- $p_{T}$ results are unaffected by the different approaches and all agree with the available experimental data $[312,313]$ within errors for both $p$ and $d$, validating the transverse dynamics of the calculations. Note that this confirms the previous findings in [184], where the hybrid approach employed was carefully constrained by an extensive experimental data set.

Figure 5.34 presents the results for the elliptic flow $\left(v_{2}\right)$ for $p$ and $d$ for the same six centrality classes, as a more sensitive probe of the momentum distribution. Due to limited statistics of the calculation, only the integrated $v_{2}$ is presented, which still allows to contrast the different approach for the $d$ reactions. Even though no experimental data is available for this observable, the order of magnitude of around 0.1 is comparable to the $p_{T}$ dependent $v_{2}$ reported in [314]. Regarding the integrated $v_{2}$ of $d$ in the bottom panel of the figure, the different reaction treatments are found to have an effect. While for central collisions, they agree, the elliptic flow is decreased for the 3-to-2 reactions and by employing the stochastic criterion for more peripheral collisions. The latter is also found for the protons in the upper panel. The additional effect for 2-to-2 reactions for the two 

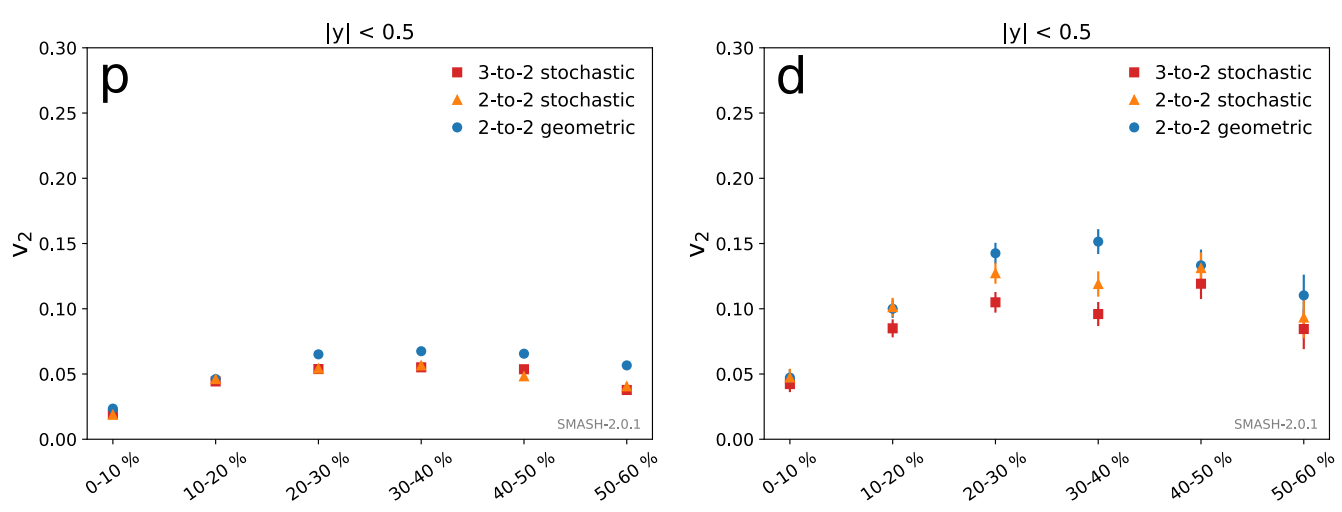

Figure 5.34: Integrated elliptic flow for protons (top) and deuterons (bottom).

different collision criteria might hint at limitations of the stochastic criterion for the small systems in peripheral collisions. However, a more uniform, thermalized medium and a subsequent lower $v_{2}$ might also be expected when employing multi-particle reactions. In

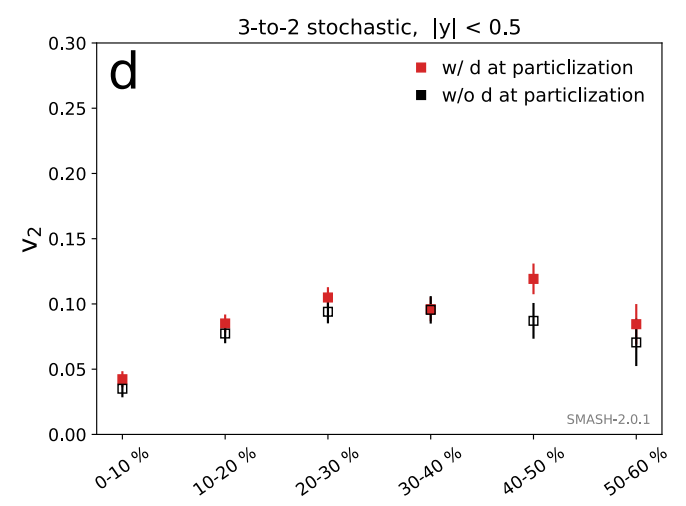

Figure 5.35: Integrated elliptic flow for deuterons with and without d at particlization.

Figure 5.35 the impact of $d$ solely being produced in the afterburner on the elliptic flow is studied. Observing a clear difference here would potentially allow disentangling the two pictures for $d$ production times. Even though a clear difference is not found, a small decrease is seen for the case without $d$ at particlization. The difference is not significant for all centrality classes; the effect for the average over all centralities, however, is significant.

\section{Summary}

This study represents the second application of multi-particle reactions in the hadronic transport approach SMASH and the first results that show the significance of multi-particle reactions for current research questions. For the deuterons, multi-particle reactions are of major importance since they treat the catalysis reactions while fulfilling detailed balance. The stochastic collision criterion avoids introducing an artificial resonance by treating the deuteron $3 \rightarrow 2$ catalysis reactions in one step.

The deuteron production in gold-gold collisions at a beam energy of $7.7 \mathrm{GeV}$ is studied in a hybrid approach, where the deuteron yield agrees with the experimental data whether the nuclei are produced at the time of particlization or not. This explains the apparent contradiction that thermal and coalescence models reproduce the $d$ yield. The "snowballs in hell" do not survive but are constantly destroyed and (re-)formed, keeping the $d$ yield 
constant. And, if no $d$ are initialized early, they are generated at later times, like assumed in coalescence approaches. A yield similar to the thermal model yield is generated in this case. These findings confirm the previous studies of employing the slower equilibrating two-body reaction chain involving the fake $d^{\prime}$ resonance $[183,184]$.

Even though the interpretation of the results is the same for the 3-to-2 and 2-to-2 treatment, some differences are observed. The faster equilibration process of the multi-particle reactions leads to a more rapid increase in the deuteron yield before the system freezes out chemically due to the system's expansion. The $d$ yield is consequently enhanced. The difference in the final number of $d$, when comparing the scenarios of $d$ being produced in the hydrodynamic stage or just in the hadronic afterburner, is thereby greatly reduced when employing multi-particle reactions due to three-body reactions driving the $d$ faster to statistical equilibrium. The reduced difference when employing 3-to-2 in comparison to 2 -to- 2 reactions is attributed to the different times required to equilibrate already found in box calculations (Figure 5.9), which is significantly reduced for the 3 -to- 2 treatment, as predicted by rate equations.

As an additional result, a decrease in the elliptic flow is found when employing the stochastic criterion and multi-particle reactions for more peripheral collisions. Similarly, a small decline in flow for all centralities is reported if all $d$ are produced during the collision's late (afterburner) stages. No dependency is found for mean transverse momentum, where an agreement with experimental data for all centrality classes is seen. 


\subsection{Proton-antiproton regeneration in the late collision stages}

This section is based on [5].

The effect of proton-antiproton annihilation $(\mathrm{p} \overline{\mathrm{p}} \rightarrow 5 \pi)$ on the (anti-) proton yield in the late stages of heavy-ion collisions, while respecting detailed balance for the annihilation reaction, is investigated in this section. Stochastic multi-particle reactions are utilized to include the 5-body back-reactions for the first time in a full microscopic transport description. This allows to simultaneously quantify the effect of (anti-) protons lost due to annihilation, the amount of (anti-) protons regenerated by the back-reaction and the result of this interplay on the final yields. The long-standing question about the p anomaly (Section 1.3.4) is addressed. The presented study builds on different previous works, which showed the significance of baryon-anti-baryon annihilation reactions in the late collision stages $[66,67,192,193]$. Specifically clarifying the role of the (5-body) backreaction is a motivation, since, as discussed in Section 1.3.4, the few available theoretical calculations disagree on the factor and, therefore, significance of regeneration. The number of regeneration reactions relative to annihilation ranges from $20 \%$ to over $100 \%$ [194, 131, 129, 130]. A full regeneration would mean that no $\mathrm{p}$ are depleted by proton-antiproton annihilations in the late collisions stages and rule out the reaction as a possible explanation for the p anomaly.

The heavy-ion collision systems studied to quantify the effect of annihilations, are $\mathrm{PbPb}$ and $\mathrm{AuAu}$ at an energy of $\sqrt{s_{N N}}=17.3 \mathrm{GeV}, \sqrt{s_{N N}}=200 \mathrm{GeV}$ and $\sqrt{s_{N N}}=5.02$ $\mathrm{TeV}$, as measured at RHIC and LHC. The theoretical approach is a hybrid model. The focus of the displayed results is on the transport afterburner calculation for the late nonequilibrium stages, which includes the $\mathrm{p} \overline{\mathrm{p}} \leftrightarrow 5 \pi$ reactions. The employed hybrid approach is the SMASH-vHLLE-Hybrid [237], which embeds the viscous 3+1D viscous hydrodynamic evolution of vHLLE [91] into the SMASH transport approach. The initial conditions and the afterburner phase are calculated using SMASH [98, 213]. Particlization as the initial conditions for the afterburner is achieved with the SMASH-hadron-sampler $[124,236]$. For the initial conditions, the SMASH evolution is performed until nuclear overlap signified by a constant proper time $\left(\tau=0.5 \mathrm{fm}\right.$ for $\left.\sqrt{s_{N N}} \geq 200 \mathrm{GeV}\right)$. When particles cross the constant proper time hypersurface, gaussian smearing is applied. The initial conditions are averaged over 100 events for three centrality classes (0-5\%, 20-30\% and 30-40\%). Averaged initial conditions are sufficient to study particle yields that are not dependent on event-by-event fluctuations. The $3+1 \mathrm{D}$ hydro calculation starting from the initial conditions employs a chiral model equation of state [315]. The shear viscosity is set to $\eta / s=0.1$ and the bulk viscosity to $\zeta / s=0.05$ for all collision energies. As the switching condition for particlization, an energy density of $\epsilon_{\text {crit }}=0.5 \mathrm{GeV} / \mathrm{fm}^{3}$ is chosen [316]. The SMASH-hadron-sampler generates 2000 events from the resulting $\epsilon_{\text {crit }}$-hypersurface, particlizing the fluid elements for the afterburner calculation. Overall, the hybrid approach is not tuned to precisely describe experimental data but employs parameters that are known $[191,78]$ to reproduce bulk properties well.

In the following, the regeneration of (anti-) protons as the species of most interest is studied, which serve as a proxy for all $B \bar{B}$ annihilation reactions. Even though the $n \bar{n} \leftrightarrow$ $5 \pi$ reactions are part of the calculations, the $n$ yield is not discussed since it is difficult to measure. All $B \bar{B}$ annihilation (except $\mathrm{NN}$ ) are realized via string fragmentation, where the back-reaction is unaccounted for and detailed balance is broken. Note that for the results labeled as no back-reaction also NN is realized via string fragmentation, which resembles 
previous theoretical approaches $[66,67,192,193]$ that only account for the annihilation, but not the regeneration reaction. The two detailed balance fulfilling treatments for the $5 \leftrightarrow 2$ reactions are introduced in Section 4.3.3. The direct treatment of the forward and backward $5 \leftrightarrow 2$ reaction via the stochastic collision criterion is referred to as the stochastic treatment in the following, as it is only possible with the stochastic criterion. The other treatment handles the same overall reaction via intermediate resonances as a chain of twobody reactions ( $\mathrm{NN} \rightarrow \mathrm{h}_{1} \rho \rightarrow \rho \pi \pi \pi \rightarrow 5 \pi$ ). This is computationally less intensive and applicable with the geometric collision criterion, which only allows to conserve detailed balance for two-body reactions. The treatment is referred to as the resonance treatment in the following. The results for the resonance treatment are calculated using the geometric collision criterion ${ }^{11}$. The comparison of both $5 \leftrightarrow 2$ treatments, stochastic and resonance, allows gauging the effects and validity of employing multi-step reaction chains involving resonances with finite lifetimes for multi-particle reactions. The stochastic treatment builds on the previous work in [128], where proton-antiproton annihilations reactions are studied as well. However, only $3 \leftrightarrow 2$ reactions are used, where the $5 \pi$ final state is created by resonance decays from reactions like $\mathrm{p} \overline{\mathrm{p}} \rightarrow \rho \rho \pi$. This is comparable to the resonance treatment used here. Note that proton-antiproton annihilations in this study only produce a $5 \pi$ final state as an effective approach and average of the other possible $\mathrm{p} \overline{\mathrm{p}} \rightarrow m \pi$ with $m=2,3 \ldots$ reactions. In the typical energy range of the p $\overline{\mathrm{p}}$ scattering (close to the two-nucleon mass threshold) in the afterburner evolution, $m=5$ is known to be the most likely result of the annihilation reaction [304]. The limitation to $m=5$ also allows directly comparing the resonance with the stochastic approach since the former does not allow other final states.

The following results rely on the presented verification of the 5-to-2 treatments in Section 5.1.2. The box calculations verify the equilibrium properties. In particular, the comparison to the analytic evolution [305] gauges that the equilibrated yield and the equilibration process are correct for the stochastic treatment. It is also found that employing multi-particle (stochastic) reactions leads to a faster equilibration of the medium in comparison to the resonance treatment, which is compatible with findings for 3-to-2 and 3-to-1 reactions (Section 5.1.2). For the deuteron 3-to-2 reactions, it is furthermore shown in the previous result section (Section 5.3) that the differences of equilibration time in box calculations relate to different particle yields in the afterburner calculation. Whether a similar relation is also seen for the proton 5 -to-2 reaction is studied in the following.

\section{Results}

The first results show the reaction rate in the afterburner calculation for the two treatments accounting for the $5 \pi \rightarrow \mathrm{p} \overline{\mathrm{p}}$ annihilation back-reaction at different energies (Figure 5.36). This allows to gauge whether the longer reaction time or re-scatterings within the medium of the intermediate resonance of the resonance treatment affects the results. Note that in the case of the resonance treatment, only the $\mathrm{h}_{1} \rho \leftrightarrow \mathrm{p} \overline{\mathrm{p}}$ is tracked as the annihilation forward and backward reaction since subsequent reaction steps mix with the medium. Both treatments appear to be equivalent for forward and backward reaction rate $(\mathrm{p} \overline{\mathrm{p}}$ anni. and prod.), which is a surprising difference to the reported result of the box calculations in Section 5.1.2. The different equilibration of the two $5 \pi \leftrightarrow p \bar{p}$ treatments has a negligible effect on the studied expanding full hadron gas. In contrast, the $d$ catalysis reactions at lower beam energies revealed differences between multi-step and multi-particle treatments (previous Section 5.3). A possible explanation could be that the, compared to lower beam

\footnotetext{
${ }^{11}$ Using the stochastic criterion would be equivalent as the box calculation in Figure 5.10b shows.
} 

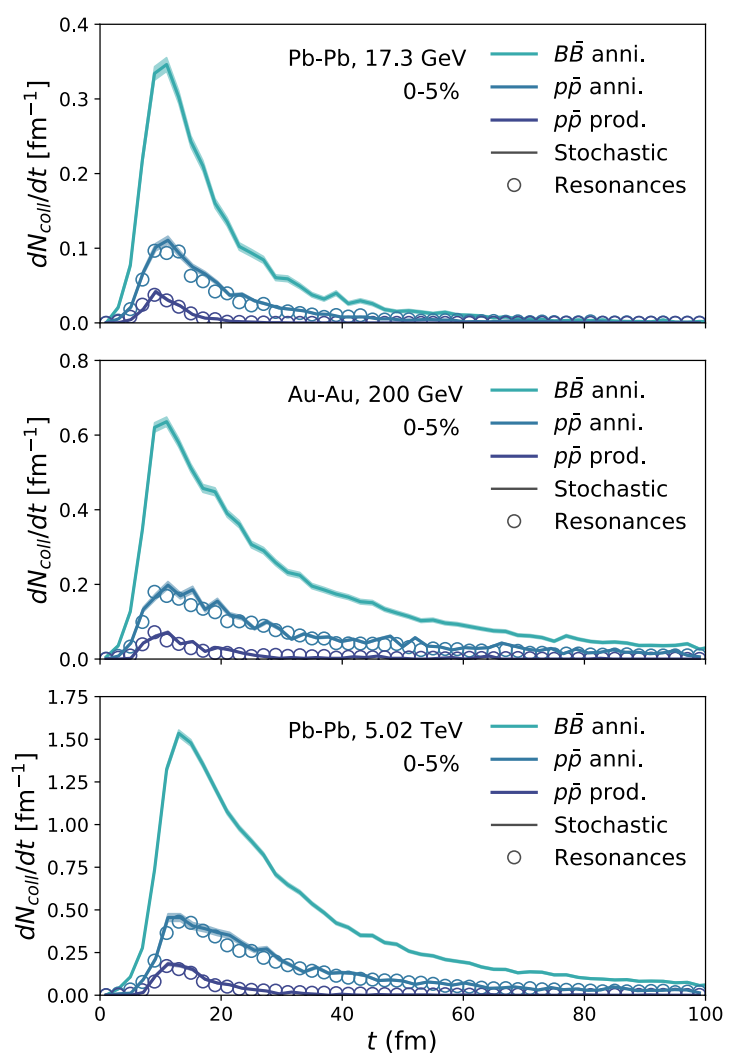

Figure 5.36: Reaction rates of annihilation (anni.) and back-reaction (prod.) for stochastic and resonance treatment for different collision systems and energies. Figure from [5].

energies, larger and longer-lived $\pi$-rich medium minimizes effects of a slower reaction. So, while the stochastic treatment is more flexible and rigorous and thereby lends credibility, it appears that the 5-to- 2 reaction can be modeled in multiple steps without affecting the reported afterburner results. Figure 5.36 also displays that the $\mathrm{B} \overline{\mathrm{B}}$ annihilation rate ${ }^{12}$ is not saturated by $\bar{p} \bar{p}$, which shows that also other baryon annihilation reactions are substantial for the reaction dynamics.

In order to quantify the abundance of the $\mathrm{p} \overline{\mathrm{p}}$ annihilation back-reaction (BR) in the full microscopic description of the late collision stages, the reaction rate is integrated to obtain the total reaction numbers $\left(\mathrm{N}_{\text {coll }}\right)$. A monotonic rise with incident energy $\sqrt{s_{N N}}$ is found (Figure 5.37, top). The p $\bar{p}$ back-reaction contribution is $15-20 \%$ relative to all $\mathrm{p} \overline{\mathrm{p}}$ annihilations (Figure 5.37, bottom). Remarkably, this fraction is constant over the full range of energies $\left(\sqrt{s_{N N}}=0.0175-5.02 \mathrm{TeV}\right)$, and centralities $(0-50 \%)$ that are investigated. Similarly, the ratio of $\mathrm{p} \overline{\mathrm{p}}$ relative to $\mathrm{B} \overline{\mathrm{B}}$ is constant and ranges between $25 \%$ and $30 \%$. This can be used to extrapolate the total amount of annihilation and regeneration of $\mathrm{B} \overline{\mathrm{B}}$ pairs by assuming $\mathrm{p} \overline{\mathrm{p}}$ as a proxy for $\mathrm{B} \overline{\mathrm{B}}$. As the rates match (cf. Figure 5.36), only results obtained with one of the reaction treatments is displayed in Figure 5.37 (in this case, the resonance approach).

The inclusion of back-reactions in the afterburner calculations finally enables to study of the effect of annihilation and regeneration on the particle yield itself. Three scenarios are

\footnotetext{
${ }^{12}$ No comparison between treatments is displayed for $\mathrm{B} \overline{\mathrm{B}}$ annihilations since they always proceed via string fragmentation.
} 


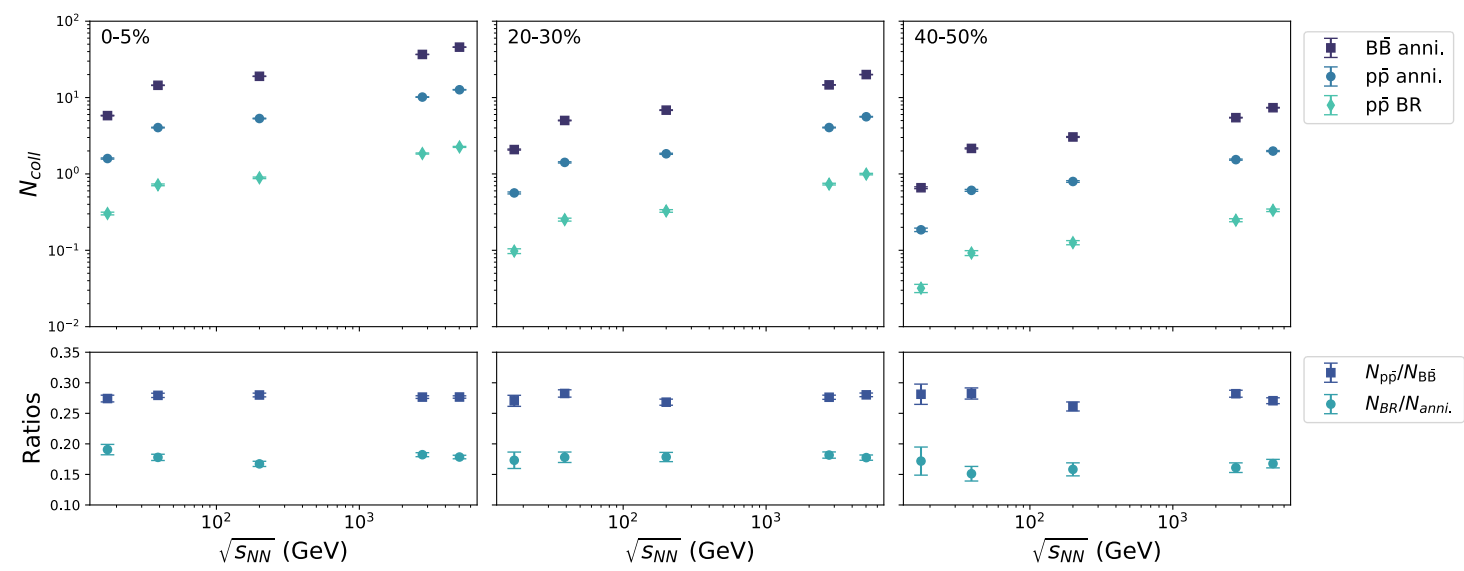

Figure 5.37: Top: Reaction numbers for $\mathrm{B} \overline{\mathrm{B}}$ and $\mathrm{p} \overline{\mathrm{p}}$ annihilation and back-reaction (BR). Bottom: Reaction number ratio of $p \bar{p}$ over $\mathrm{B} \overline{\mathrm{B}}$ annihilation and $\mathrm{p} \overline{\mathrm{p}}$ back-reaction over annihilation. Results for different systems $\left(\mathrm{Au}-\mathrm{Au}, \sqrt{s_{N N}}=39,200 \mathrm{GeV}, \mathrm{Pb}-\mathrm{Pb}, \sqrt{s_{N N}}=\right.$ $17.3 \mathrm{GeV}, 2.76$ and 5.02 TeV.) and different centrality classes (0-5, 20-30 and 40-50\%). Figure from [5].

distinguished in the calculations: (i) Performing only decays after particlization allows to account for the feed down from resonance decays but neglects any rescattering effect. This is the same picture of particle production as assumed by thermal models $[188,190]$. (ii) Including no back-reaction shows the maximal depletion effect of proton-antiproton annihilations on the $\mathrm{p}(\overline{\mathrm{p}})$ yield and is already studied with comparable approaches in $[67$, 317, 193]. (iii) The $5 \pi \rightarrow \mathrm{p} \overline{\mathrm{p}}$ back-reaction is taken into account to respect detailed balance for the annihilation reaction and quantify the regeneration effect. First, the $4 \pi$ yield ${ }^{13}$ is considered for the three scenarios (Figure 5.38). The maximum annihilation rescattering effect is found as the difference between the results with only decays and with no back-reaction (dotted and dashed lines). A clear energy dependence is observed. The significance of annihilations is larger at higher energies. Also, the p yield is more depleted than the $\overline{\mathrm{p}}$ yield. The regenerated yield (solid lines) is consistent with the found ratio between back- and annihilation reaction of $15-20 \%$, which is consequential since the reaction number are also counted for the full phase space $(4 \pi)$. Notably, the backreaction does not regenerate the full yield lost by annihilations (i.e. the solid line is not consistent with the dotted line). Finally, also for the yield, no significant effect of employing either the resonance or the stochastic reaction treatment is observed. The phase space region of most interest sits at mid-rapidity, as the created medium is centered in this region and is unaffected by spectators. Also, current high-energy experiments are collider setups, so most of their (proton) measurements are only available in this region. Therefore, the annihilation and regeneration effect of most interest is on the mid-rapidity yield (Figure 5.39). Including rescattering for (anti-) protons by annihilation (without back-reactions) lowers the mid-rapidity yield ${ }^{14}$ compared to only accounting for the decays.

\footnotetext{
${ }^{13}$ The yield without any kinematic restrictions on the phase space of the final particles is referred to as $4 \pi$ yield. The name stems from the integration over all angles.

${ }^{14}$ Note that while the difference between the $\mathrm{p}$ and $\overline{\mathrm{p}}$ yield decreases with the higher energies, a small difference remains even at $\sqrt{s_{N N}}=5.02 \mathrm{TeV}$. This is in contrast to experimental measurements that find $\mathrm{p}$ and $\overline{\mathrm{p}}$ yields compatible at these energies. The difference is caused by a small finite positive $\mu_{B}$ value at particlization, which can be improved upon by updated initial conditions. As the aim of this study is not to precisely describe experimental data, improving the initial condition is left for future work.
} 

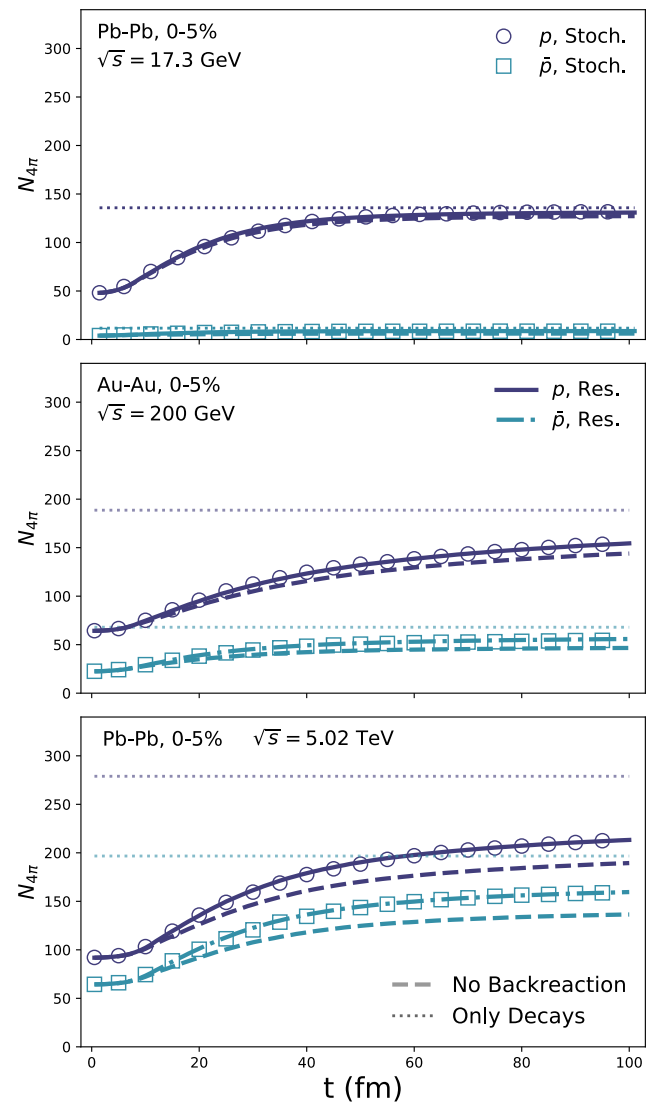

Figure 5.38: Time evolution of $\mathrm{p}$ and $\overline{\mathrm{p}}(4 \pi)$ yields. Figure from [5].

The agreement with experimental data $[318,319]$ is thereby improved. Including the backreactions regenerates about $50 \%$ of the $\mathrm{p}(\overline{\mathrm{p}})$ yield at mid-rapidity lost by annihilations for all investigated energies. Even though the effect of regeneration is sizable, still a netreduction of the $\mathrm{p}(\overline{\mathrm{p}})$ yield is found when fulfilling detailed balance for $5 \pi \leftrightarrow \mathrm{p} \overline{\mathrm{p}}$, which is favored by the available experimental data. The effect of the back-reactions is enhanced at mid-rapidity compared to $4 \pi$.

The reported findings agree with previous studies with the comparable UrQMD approach $[192,193,320]$, as a decrease of the (anti-) proton yield, which improves the agreement with experimental data, is observed even when treating the annihilation reaction with detailed balance. In particular, no full regeneration of the yield as reported in [131] is observed. The expansion appears to supersede the backward reaction probability. The results agree with reports employing rate equation in an expanding volume [194]. Remarkably, the authors also report a regeneration of $20 \%$ on the $(4 \pi)$ yield when including back-reactions, which is the same percentage reported in this work. Lastly, comparing with the results of the PHSD approach [130] that treat a more extensive set of $\mathrm{B} \overline{\mathrm{B}}$ reactions via detailed balance conserving 3-to-2 reactions, a similar net reduction of protons at $\sqrt{s_{N N}}=200 \mathrm{GeV}$ is found. Conversely, the net enhancement reported at $\sqrt{s_{N N}}=5.02$ $\mathrm{TeV}$ disagrees with the findings here. 

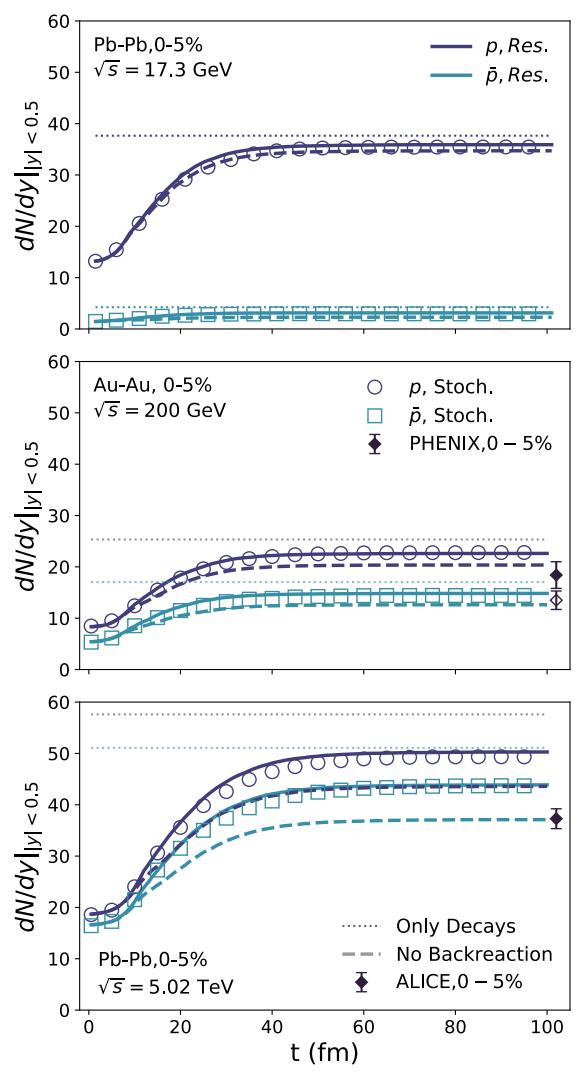

Figure 5.39: Time evolution of $\mathrm{p}$ and $\overline{\mathrm{p}}$ mid-rapidity yields. Experimental data from [318, 319]. Figure from [5].

\section{Summary}

This section reports the first results for 5-body annihilation back-reactions in a transport approach for the late non-equilibrium heavy-ion collision phase. $\mathrm{p} \overline{\mathrm{p}} \rightarrow 5 \pi$ annihilation reactions are included in the calculation in accordance with the principle of detailed balance by also treating the $5 \pi \rightarrow \mathrm{p} \overline{\mathrm{p}}$ back-reaction. This allows to address how significant the proton annihilation reaction depletes and regenerates the $\mathrm{p}(\overline{\mathrm{p}})$ yield. The fraction of back-reactions relative to annihilations is found to be $20 \%$ over all studied energies and centralities. Consequently, the total $(4 \pi)$ yield is $20 \%$ regenerated by the back-reaction. The back-reaction effect is enhanced at mid-rapidity, where about half of the $\mathrm{p}(\overline{\mathrm{p}})$ yield is regenerated. For the reported results, treating the back-reaction directly via stochastic 5 -to-2 reaction or in multiple binary reaction steps is equivalent. Note, however, that only the stochastic treatment can flexibly account for all reactions of the form $\mathrm{p} \overline{\mathrm{p}} \rightarrow m \pi$ and further baryon-anti-baryon annihilation reactions in future studies. Overall, both forward and backward annihilation reactions are found to be important non-equilibrium effects on the proton yield. In particular, the sizable regeneration is important for the extraction of transport coefficients [191, 78] or the switching temperature of hybrid approaches [133], which are sensitive to the final proton yield. The results, in general, also underline the significance of a non-equilibrium rescattering phase towards the end of a heavy-ion collision. The assumption of an early chemical freeze-out at a common temperature and chemical potential for all particle species is not supported, even when employing multi-particle reactions to fulfill detailed balance for the proton-antiproton annihilation. 



\section{Chapter 6}

\section{Conclusion and outlook}

The overall addressed question in this work is: How does the hadronic medium evolve in heavy-ion collisions i.e. what are the relevant microscopic reaction mechanisms and the properties of the involved degrees of freedom? The main goal is to address this question specifically for hadronic multi-particle interactions. For this goal, the hadronic transport approach SMASH is extended with stochastic rates, which allow to include detailed balance fulfilling multi-particle reactions in the approach. Three types of reactions are newlyaccounted for: 3 -to-1, 3-to-2 and 5-to-2 reactions. After extensive verifications of the stochastic rates approach, they are used to study the effect of multi-particle interactions, particularly in afterburner calculations. These studies follow complementary results for the dilepton and strangeness production of the hadronic transport approach employing only binary reactions. The overarching conclusions obtained in those two avenues of study, for binary and multi-particle reactions, are given together with an outlook for possible future studies in this chapter.

Hadronic transport approaches with binary reactions are found to be capable of describing observables when employed for the entire evolution of low-energy heavy-ion collisions. This is illustrated by the agreement of dilepton and strangeness production for smaller systems with SMASH calculations, which also specifically verifies the applied transport approach. It is, in particular, possible to match the measured strangeness production of $\phi$ and $\Xi$ hadrons via additional heavy $N^{*}$ decay channels, also in larger systems. The predicted production for upcoming measurements will allow to test this production mechanism.

For larger systems or higher energies, hadronic transport cascade calculations with vacuum resonance properties can point out additional medium effects. This is demonstrated extensively for the dilepton emission in comparisons to the full set of HADES dielectron data. The dilepton invariant mass spectra are sensitive to a medium modification of the vector meson spectral function for large collision systems already at low beam energies. Such a change of the spectral function is predicted for an onset of chiral symmetry restoration. The sensitivity to medium modifications is mapped out theoretically in detail by comparisons to a coarse-graining approach, which employs medium-modified spectral functions and is based on the same evolution.

The theoretical foundation of (multi-particle) stochastic rates are collision probabilities derived from the Boltzmann equation's collision term with the assumption of a constant matrix element. The derivation is presented in a comprehensive and pedagogical fashion and includes results for binary, 3- and 5-body reactions. The derived collision probabili- 
ties are employed for a stochastic collision criterion and various detailed-balance fulfilling multi-particle reactions: the mesonic Dalitz decay back-reaction (3-to-1), the deuteron catalysis (3-to-2 ) and the proton-antiproton annihilation back-reaction (5-to-2). The introduced stochastic rates approach is extensively verified by studies of the numerical stability and comparisons to previous results and analytic expectations. The stochastic rates results agree perfectly with the respective analytic results. This work represents the first successful application of a stochastic criterion and direct multi-particle reactions in SMASH.

Physically, multi-particle reactions are demonstrated to be significant for different observables, most notably the yield of the partaking particles, even in the late dilute stage of heavy-ion reactions. They lead to a faster equilibration of the system than equivalent binary multi-step treatments. The difference in equilibration consequently influences the yield in afterburner calculations. Interestingly, the interpretation of results is not dependent on employing multi-particle or multi-step treatments, which a posteriori validates the latter. Stochastic multi-particle reactions are still preferable, even though numerically more challenging since they allow treating the reactions directly on a theoretically rigorous and fully flexible basis, without the need to introduce, partly poorly motivated, multi-step helper constructs. As the first test case of multi-particle reactions in heavy-ion reactions, the mesonic 3-to-1 Dalitz decay is found to be dominated by the $3 \pi \rightarrow \omega$. While the effect on the medium is found to be negligible overall, the regeneration is found to be sizable: up to a quarter of Dalitz decays are regenerated.

Non-equilibrium rescattering effects are shown to be relevant for late collision stages for two-particle species: deuteron and protons. In both cases, the relevant rescatterings involve multiple particles. The deuteron $\pi$ and $N$ catalysis reactions equilibrate quickly in the afterburner stage at intermediate energies. The constant formation and destruction keeps the yield constant and microscopically explains the "snowballs in hell"-paradox. The yield is also generated with no $d$ present at early times, which explains why coalescence models can also match the multiplicity. The results with multi-particle reactions are therefore able to confirm previous studies with an artificial multi-step treatment. New is the study of the 5-body back-reaction of proton-antiproton annihilations. This work marks the first realization of microscopic 5-body reactions in a transport approach to fulfill detailed balance for $p \bar{p} \leftrightarrow 5 \pi$. A sizeable regeneration due to the back-reaction of up to half of the proton-antiproton pairs lost due to annihilations is found. Consequently, both annihilation and regeneration in the late non-equilibrium stage are shown to have a significant effect on the $p$ yield. 


\section{Outlook}

A significant achievement, besides the mentioned physical results, is the establishment of a new general framework for stochastic rates that is flexible to account for any $n \rightarrow m$ reactions, with $m=1,2$, in future studies. The presented general derivations, verifications, and explanations are given to facilitate such extensions. Of particular research interest are extensions of the $d$ and $p$ studies with more reactions that adhere to detailed balance. The $d$ study would be complemented by considering other light nuclei like (hyper-)triton and helium. While the conclusions employing the 2-to-2 reaction treatment involving the artificial $d^{\prime}$ are confirmed by this work, introducing additional fake resonances like $t^{\prime}$ would be tedious and enhance the found differences to the theoretically preferable direct 3 -to- 2 treatment. Also, the study of annihilation reactions could be extended by considering other $B \bar{B}$ (back-)reactions. Multi-particle reactions involving strangeness are known [129] as well and could be explored. For the multi-particle framework in this work, all reactions have to be introduced individually. Detailed balance is therefore not fulfilled globally in all calculations due to the string fragmentation processes. Following the principle of detailed balance globally would be theoretically desirable and requires a generic treatment of the multi-particle back-reactions. One of the major challenges is the non-deterministic forward direction of the string fragmentation. An idea related to a global back-reaction treatment is the statistical determination of the unknown forward cross-sections [321].

SMASH has been used extensively for the study of transport coefficients [204, 205, 207, 206]. Therefore, the effect of multi-particle interactions on the different transport coefficients, particularly the shear viscosity and baryon diffusion, would be interesting to investigate. Here, the comparison between instantaneous multi-particle and slower multistep approaches is interesting since it has been demonstrated in [204] that the lifetime affects the coefficients. Also, a detailed investigation of the Lorentz-invariance of the different criteria [223] is omitted and left for future work. Several of the mentioned (future) studies would benefit and maybe even require further optimization of the calculation runtime. Different options for performance optimizations are given for this purpose in Appendix A.4.

Regarding the results with binary reactions, comparing the calculated predictions for dileptons and double strange hadrons to upcoming experimental data will provide further inside into the sensitivity to medium modification of dilepton emission and the strangeness production mechanism. In the future, the dilepton production in intermediate and high beam energy collisions could be addressed based on the presented well-understood baseline at low beam energies. A hybrid dilepton approach would allow to explore high-beam energy reactions of RHIC or LHC. Here, the dilepton production of SMASH for the hadronic afterburner stage could be combined with the dilepton radiation from a hydrodynamic calculation. Since dilepton emission remains a key observable for high-density collisions at upcoming facilities, like CBM at FAIR, such a hybrid approach could also be applied to intermediate beam energy collisions.

Overall, the results of this work provide many conductive points of reference to continue the study of the hadron gas phases in heavy-ion collisions. 



\section{Bibliography}

[1] J. Staudenmaier, J. Weil, V. Steinberg, S. Endres, and H. Petersen, "Dilepton production and resonance properties within a new hadronic transport approach in the context of the GSI-HADES experimental data," Phys. Rev. C, vol. 98, no. 5, p. $054908,2018,1711.10297$.

[2] V. Steinberg, J. Staudenmaier, D. Oliinychenko, F. Li, Ö. Erkiner, and H. Elfner, "Strangeness production via resonances in heavy-ion collisions at energies available at the GSI Schwerionensynchrotron," Phys. Rev., vol. C99, no. 6, p. 064908, 2019, 1809.03828 .

[3] J. Staudenmaier, N. Kübler, and H. Elfner, "Particle production in AgAg collisions at $E_{\text {Kin }}=1.58 \mathrm{~A} \mathrm{GeV}$ within a hadronic transport approach," Phys. Rev. C, vol. 103, no. 4, p. 044904, 2021, 2008.05813.

[4] J. Staudenmaier, D. Oliinychenko, J. M. Torres-Rincon, and H. Elfner, ""Smashing more than two": Deuteron production in relativistic heavy ion collisions via stochastic multi-particle reactions," 6 2021, 2106.14287.

[5] O. Garcia-Montero, J. Staudenmaier, A. Schäfer, J. M. Torres-Rincon, and H. Elfner, "The role of proton-antiproton regeneration in the late stages of heavy-ion collisions," 2021, 2107.08812.

[6] C. MissMJ, "Elementary particles included in the standard model." https://en. wikipedia.org/wiki/Elementary_particle. [Online; accessed 28-Juli-2021].

[7] S. K. Choi et al., "Observation of a resonance-like structure in the $p i^{ \pm} \psi^{\prime}$ mass distribution in exclusive $B \rightarrow K \pi^{ \pm} \psi^{\prime}$ decays," Phys. Rev. Lett., vol. 100, p. 142001, 2008, 0708.1790.

[8] R. Aaij et al., "Observation of $J / \psi p$ Resonances Consistent with Pentaquark States in $\Lambda_{b}^{0} \rightarrow J / \psi K^{-} p$ Decays," Phys. Rev. Lett., vol. 115, p. 072001, 2015, 1507.03414.

[9] D. J. Gross and F. Wilczek, "Ultraviolet behavior of non-abelian gauge theories," Phys. Rev. Lett., vol. 30, pp. 1343-1346, Jun 1973.

[10] H. D. Politzer, "Reliable perturbative results for strong interactions?," Phys. Rev. Lett., vol. 30, pp. 1346-1349, Jun 1973.

[11] S. Bethke, " $\alpha_{s}$ at Zinnowitz 2004," Nucl. Phys. B Proc. Suppl., vol. 135, pp. 345-352, 2004, hep-ex/0407021.

[12] G. Aarts, "Introductory lectures on lattice QCD at nonzero baryon number," $J$. Phys. Conf. Ser., vol. 706, no. 2, p. 022004, 2016, 1512.05145. 
[13] A. M. Halasz, A. D. Jackson, R. E. Shrock, M. A. Stephanov, and J. J. M. Verbaarschot, "On the phase diagram of QCD," Phys. Rev. D, vol. 58, p. 096007, 1998, hep-ph/9804290.

[14] M. A. Stephanov, K. Rajagopal, and E. V. Shuryak, "Signatures of the tricritical point in QCD," Phys. Rev. Lett., vol. 81, pp. 4816-4819, 1998, hep-ph/9806219.

[15] J. Berges and K. Rajagopal, "Color superconductivity and chiral symmetry restoration at nonzero baryon density and temperature," Nucl. Phys. B, vol. 538, pp. 215232, 1999, hep-ph/9804233.

[16] R. Rapp, T. Schäfer, E. V. Shuryak, and M. Velkovsky, "High density QCD and instantons," Annals Phys., vol. 280, pp. 35-99, 2000, hep-ph/9904353.

[17] N. Armesto and E. Scomparin, "Heavy-ion collisions at the Large Hadron Collider: a review of the results from Run 1," Eur. Phys. J. Plus, vol. 131, no. 3, p. 52, 2016, 1511.02151.

[18] J. Adams et al., "Experimental and theoretical challenges in the search for the quark gluon plasma: The STAR Collaboration's critical assessment of the evidence from RHIC collisions," Nucl. Phys. A, vol. 757, pp. 102-183, 2005, nucl-ex/0501009.

[19] K. Adcox et al., "Formation of dense partonic matter in relativistic nucleus-nucleus collisions at RHIC: Experimental evaluation by the PHENIX collaboration," Nucl. Phys. A, vol. 757, pp. 184-283, 2005, nucl-ex/0410003.

[20] K. Aamodt et al., "The ALICE experiment at the CERN LHC," JINST, vol. 3, p. S08002, 2008.

[21] R. Arnaldi et al., "J/psi production in indium-indium collisions at 158GeV/nucleon," Phys. Rev. Lett., vol. 99, p. 132302, 2007.

[22] B. Friman, C. Hohne, J. Knoll, S. Leupold, J. Randrup, R. Rapp, and P. Senger, "The CBM physics book: Compressed baryonic matter in laboratory experiments," Lect. Notes Phys., vol. 814, pp. pp.1-980, 2011.

[23] G. Agakishiev et al., "The High-Acceptance Dielectron Spectrometer HADES," Eur. Phys. J. A, vol. 41, pp. 243-277, 2009, 0902.3478.

[24] P. Spiller and G. Franchetti, "The FAIR accelerator project at GSI," Nucl. Instrum. Meth. A, vol. 561, pp. 305-309, 2006.

[25] V. Kekelidze, R. Lednicky, V. Matveev, I. Meshkov, A. Sorin, and G. Trubnikov, "NICA project at JINR," Phys. Part. Nucl. Lett., vol. 9, pp. 313-316, 2012.

[26] H. Sako et al., "Towards the heavy-ion program at J-PARC," Nucl. Phys., vol. A931, pp. 1158-1162, 2014.

[27] L. Adamczyk et al., "Energy Dependence of Moments of Net-proton Multiplicity Distributions at RHIC," Phys. Rev. Lett., vol. 112, p. 032302, 2014, 1309.5681.

[28] I. Arsene et al., "Quark gluon plasma and color glass condensate at RHIC? The Perspective from the BRAHMS experiment," Nucl. Phys. A, vol. 757, pp. 1-27, 2005, nucl-ex/0410020.

[29] B. B. Back et al., "The PHOBOS perspective on discoveries at RHIC," Nucl. Phys. $A$, vol. 757, pp. 28-101, 2005, nucl-ex/0410022. 
[30] E. Shuryak, "Physics of Strongly coupled Quark-Gluon Plasma," Prog. Part. Nucl. Phys., vol. 62, pp. 48-101, 2009, 0807.3033.

[31] C. Adler et al., "Disappearance of back-to-back high $p_{T}$ hadron correlations in central $\mathrm{Au}+\mathrm{Au}$ collisions at $\sqrt{s_{N N}}=200-\mathrm{GeV}$," Phys. Rev. Lett., vol. 90, p. 082302, 2003, nucl-ex/0210033.

[32] B. Muller and J. L. Nagle, "Results from the relativistic heavy ion collider," Ann. Rev. Nucl. Part. Sci., vol. 56, pp. 93-135, 2006, nucl-th/0602029.

[33] S. Chatrchyan et al., "Observation of a New Boson at a Mass of $125 \mathrm{GeV}$ with the CMS Experiment at the LHC," Phys. Lett. B, vol. 716, pp. 30-61, 2012, 1207.7235.

[34] G. Aad et al., "Observation of a new particle in the search for the Standard Model Higgs boson with the ATLAS detector at the LHC," Phys. Lett. B, vol. 716, pp. 1-29, $2012,1207.7214$.

[35] P. W. Higgs, "Broken Symmetries and the Masses of Gauge Bosons," Phys. Rev. Lett., vol. 13, pp. 508-509, 1964.

[36] P. W. Higgs, "Broken symmetries, massless particles and gauge fields," Phys. Lett., vol. 12, pp. 132-133, 1964.

[37] F. Englert and R. Brout, "Broken Symmetry and the Mass of Gauge Vector Mesons," Phys. Rev. Lett., vol. 13, pp. 321-323, 1964.

[38] J. Schukraft, "QM2017: Status and Key open Questions in Ultra-Relativistic HeavyIon Physics," Nucl. Phys. A, vol. 967, pp. 1-10, 2017, 1705.02646.

[39] M. Gyulassy, I. Vitev, X.-N. Wang, and B.-W. Zhang, "Jet quenching and radiative energy loss in dense nuclear matter," 2 2003, nucl-th/0302077.

[40] C. Ratti, "Lattice QCD and heavy ion collisions: a review of recent progress," Rept. Prog. Phys., vol. 81, no. 8, p. 084301, 2018, 1804.07810.

[41] K. G. Wilson, "Confinement of Quarks," Phys. Rev., vol. D10, pp. 2445-2459, 1974.

[42] O. Philipsen, "Lattice calculations at non zero chemical potential ," PoS, vol. ConfinementVIII, p. 011, 2012.

[43] Y. Aoki, G. Endrodi, Z. Fodor, S. D. Katz, and K. K. Szabo, "The Order of the quantum chromodynamics transition predicted by the standard model of particle physics," Nature, vol. 443, pp. 675-678, 2006, hep-lat/0611014.

[44] A. Bazavov et al., "The chiral and deconfinement aspects of the QCD transition," Phys. Rev. D, vol. 85, p. 054503, 2012, 1111.1710.

[45] A. Bazavov et al., "Equation of state in ( 2+1 )-flavor QCD," Phys. Rev. D, vol. 90, p. 094503, 2014, 1407.6387.

[46] S. Borsanyi, G. Endrodi, Z. Fodor, A. Jakovac, S. D. Katz, S. Krieg, C. Ratti, and K. K. Szabo, "The QCD equation of state with dynamical quarks," JHEP, vol. 11, p. $077,2010,1007.2580$.

[47] S. Borsanyi, Z. Fodor, C. Hoelbling, S. D. Katz, S. Krieg, and K. K. Szabo, "Full result for the QCD equation of state with $2+1$ flavors," Phys. Lett. B, vol. 730, pp. 99-104, 2014, 1309.5258. 
[48] J. M. Maldacena, "The Large N limit of superconformal field theories and supergravity," Adv. Theor. Math. Phys., vol. 2, pp. 231-252, 1998, hep-th/9711200.

[49] P. Kovtun, D. T. Son, and A. O. Starinets, "Viscosity in strongly interacting quantum field theories from black hole physics," Phys. Rev. Lett., vol. 94, p. 111601, 2005, hep-th/0405231.

[50] J. Berges, N. Tetradis, and C. Wetterich, "Nonperturbative renormalization flow in quantum field theory and statistical physics," Phys. Rept., vol. 363, pp. 223-386, 2002, hep-ph/0005122.

[51] R. Alkofer and L. von Smekal, "The Infrared behavior of QCD Green's functions: Confinement dynamical symmetry breaking, and hadrons as relativistic bound states," Phys. Rept., vol. 353, p. 281, 2001, hep-ph/0007355.

[52] R. Machleidt and D. R. Entem, "Chiral effective field theory and nuclear forces," Phys. Rept., vol. 503, pp. 1-75, 2011, 1105.2919.

[53] Y. Nambu and G. Jona-Lasinio, "Dynamical model of elementary particles based on an analogy with superconductivity. i," Phys. Rev., vol. 122, pp. 345-358, Apr 1961.

[54] Y. Nambu and G. Jona-Lasinio, "Dynamical model of elementary particles based on an analogy with superconductivity. ii," Phys. Rev., vol. 124, pp. 246-254, Oct 1961.

[55] F. Gelis, E. Iancu, J. Jalilian-Marian, and R. Venugopalan, "The Color Glass Condensate," Ann. Rev. Nucl. Part. Sci., vol. 60, pp. 463-489, 2010, 1002.0333.

[56] P. Zyla et al., "Review of Particle Physics," PTEP, vol. 2020, no. 8, p. 083C01, 2020.

[57] P. Braun-Munzinger, K. Redlich, and J. Stachel, "Particle production in heavy ion collisions," in Quark-Gluon Plasma 3, pp. 491-599, World Scientifc, 2004.

[58] R. Hagedorn and K. Redlich, "Statistical Thermodynamics in Relativistic Particle and Ion Physics: Canonical or Grand Canonical?," Z. Phys. C, vol. 27, p. 541, 1985.

[59] J. Cleymans and H. Satz, "Thermal hadron production in high energy heavy ion collisions," Z. Phys. C - Particles and Fields, vol. 57, pp. 135-147, Mar. 1993.

[60] P. Braun-Munzinger, D. Magestro, K. Redlich, and J. Stachel, "Hadron production in $\mathrm{Au}-\mathrm{Au}$ collisions at RHIC," Physics Letters B, vol. 518, pp. 41-46, Oct. 2001.

[61] J. Stachel, A. Andronic, P. Braun-Munzinger, and K. Redlich, "Confronting LHC data with the statistical hadronization model," J. Phys.: Conf. Ser., vol. 509, p. 012019, May 2014.

[62] A. Andronic, P. Braun-Munzinger, and J. Stachel, "Hadron production in central nucleus-nucleus collisions at chemical freeze-out," Nuclear Physics A, vol. 772, pp. 167199, June 2006.

[63] A. Andronic, P. Braun-Munzinger, K. Redlich, and J. Stachel, "Decoding the phase structure of QCD via particle production at high energy," Nature, vol. 561, pp. 321330, Sept. 2018.

[64] P. J. Siemens and J. O. Rasmussen, "Evidence for a Blast Wave from Compressed Nuclear Matter," Phys. Rev. Lett., vol. 42, pp. 880-883, Apr. 1979.

[65] E. Schnedermann, J. Sollfrank, and U. Heinz, "Thermal phenomenology of hadrons from 200 A GeV S+S collisions," Phys. Rev. C, vol. 48, pp. 2462-2475, Nov. 1993. 
[66] K. Werner, I. Karpenko, T. Pierog, M. Bleicher, and K. Mikhailov, "Event-byEvent Simulation of the Three-Dimensional Hydrodynamic Evolution from Flux Tube Initial Conditions in Ultrarelativistic Heavy Ion Collisions," Phys. Rev. C, vol. 82, p. 044904, 2010, 1004.0805 .

[67] J. Steinheimer, J. Aichelin, and M. Bleicher, "Nonthermal p/ $\pi$ Ratio at LHC as a Consequence of Hadronic Final State Interactions," Phys. Rev. Lett., vol. 110, no. 4, p. 042501, 2013, 1203.5302.

[68] G. Agakishiev et al., "Deep sub-threshold $\mathrm{Xi}$ - production in $\mathrm{Ar}+\mathrm{KCl}$ reactions at 1.76A-GeV," Phys. Rev. Lett., vol. 103, p. 132301, 2009, 0907.3582.

[69] J. Adam et al., "Production of light nuclei and anti-nuclei in pp and $\mathrm{Pb}-\mathrm{Pb}$ collisions at energies available at the CERN Large Hadron Collider," Phys. Rev. C, vol. 93, no. 2 , p. $024917,2016,1506.08951$.

[70] A. Andronic, P. Braun-Munzinger, J. Stachel, and H. Stocker, "Production of light nuclei, hypernuclei and their antiparticles in relativistic nuclear collisions," Phys. Lett. B, vol. 697, pp. 203-207, 2011, 1010.2995.

[71] L. Rezzolla and O. Zanotti, Relativistic Hydrodynamics. Oxford University Press, Sept. 2013.

[72] J.-Y. Ollitrault, "Relativistic hydrodynamics for heavy-ion collisions," Eur. J. Phys., vol. 29, pp. 275-302, 2008, 0708.2433.

[73] E. Shuryak and I. Zahed, "High-multiplicity pp and pA collisions: Hydrodynamics at its edge," Phys. Rev. C, vol. 88, no. 4, p. 044915, 2013, 1301.4470.

[74] P. Huovinen and P. Petreczky, "QCD Equation of State and Hadron Resonance Gas," Nucl. Phys. A, vol. 837, pp. 26-53, 2010, 0912.2541.

[75] W. Israel and J. M. Stewart, "Transient relativistic thermodynamics and kinetic theory," Annals Phys., vol. 118, pp. 341-372, 1979.

[76] G. S. Denicol, E. Molnár, H. Niemi, and D. H. Rischke, "Derivation of fluid dynamics from kinetic theory with the 14-moment approximation," Eur. Phys. J. A, vol. 48, p. $170,2012,1206.1554$.

[77] D. Bazow, U. W. Heinz, and M. Strickland, "Second-order (2+1)-dimensional anisotropic hydrodynamics," Phys. Rev. C, vol. 90, no. 5, p. 054910, 2014, 1311.6720.

[78] D. Everett et al., "Phenomenological constraints on the transport properties of QCD matter with data-driven model averaging," Phys. Rev. Lett., vol. 126, no. 24, p. 242301, 2021, 2010.03928.

[79] C. Gale, S. Jeon, and B. Schenke, "Hydrodynamic modeling of heavy-ion collisions," International Journal of Modern Physics A, vol. 28, p. 1340011, Apr. 2013.

[80] C. E. Coleman-Smith, H. Petersen, and R. L. Wolpert, "Classification of initial state granularity via 2d Fourier Expansion," J. Phys. G, vol. 40, p. 095103, 2013, 1204.5774 .

[81] M. L. Miller, K. Reygers, S. J. Sanders, and P. Steinberg, "Glauber modeling in high energy nuclear collisions," Ann. Rev. Nucl. Part. Sci., vol. 57, pp. 205-243, 2007, nucl-ex/0701025. 
[82] B. Schenke, P. Tribedy, and R. Venugopalan, "Event-by-event gluon multiplicity, energy density, and eccentricities in ultrarelativistic heavy-ion collisions," Phys. Rev. $C$, vol. 86 , p. $034908,2012,1206.6805$.

[83] D. Kharzeev and E. Levin, "Manifestations of high density QCD in the first RHIC data," Phys. Lett. B, vol. 523, pp. 79-87, 2001, nucl-th/0108006.

[84] H. J. Drescher and Y. Nara, "Effects of fluctuations on the initial eccentricity from the Color Glass Condensate in heavy ion collisions," Phys. Rev. C, vol. 75, p. 034905, 2007, nucl-th/0611017.

[85] J. Weil, H. van Hees, and U. Mosel, "Dilepton production in proton-induced reactions at SIS energies with the GiBUU transport model," Eur. Phys. J., vol. A48, p. 111, 2012, 1203.3557. [Erratum: Eur. Phys. J.A48,150(2012)].

[86] H. Petersen, G.-Y. Qin, S. A. Bass, and B. Muller, "Triangular flow in event-byevent ideal hydrodynamics in $\mathrm{Au}+\mathrm{Au}$ collisions at $\sqrt{s_{\mathrm{NN}}}=200 \mathrm{~A} \mathrm{GeV}$," Phys. Rev. $C$, vol. 82, p. 041901, 2010, 1008.0625.

[87] K. Werner, I. Karpenko, M. Bleicher, T. Pierog, and S. Porteboeuf-Houssais, "Jets, Bulk Matter, and their Interaction in Heavy Ion Collisions at Several TeV," Phys. Rev. C, vol. 85, p. 064907, 2012, 1203.5704 .

[88] L. Pang, Q. Wang, and X.-N. Wang, "Effects of initial flow velocity fluctuation in event-by-event (3+1)D hydrodynamics," Phys. Rev. C, vol. 86, p. 024911, 2012, 1205.5019 .

[89] C. Gale, S. Jeon, B. Schenke, P. Tribedy, and R. Venugopalan, "Event-by-event anisotropic flow in heavy-ion collisions from combined Yang-Mills and viscous fluid dynamics," Phys. Rev. Lett., vol. 110, no. 1, p. 012302, 2013, 1209.6330.

[90] F. Cooper and G. Frye, "Comment on the Single Particle Distribution in the Hydrodynamic and Statistical Thermodynamic Models of Multiparticle Production," Phys. Rev., vol. D10, p. 186, 1974.

[91] I. Karpenko, P. Huovinen, and M. Bleicher, "A 3+1 dimensional viscous hydrodynamic code for relativistic heavy ion collisions," Comput. Phys. Commun., vol. 185, pp. 3016-3027, 2014, 1312.4160.

[92] B. Schenke, S. Jeon, and C. Gale, "(3+1)D hydrodynamic simulation of relativistic heavy-ion collisions," Phys. Rev., vol. C82, p. 014903, 2010, 1004.1408.

[93] B. Schenke, S. Jeon, and C. Gale, "Higher flow harmonics from (3+1)D event-byevent viscous hydrodynamics," Phys. Rev. C, vol. 85, p. 024901, 2012, 1109.6289.

[94] L.-G. Pang, H. Petersen, and X.-N. Wang, "Pseudorapidity distribution and decorrelation of anisotropic flow within the open-computing-language implementation CLVisc hydrodynamics," Phys. Rev. C, vol. 97, no. 6, p. 064918, 2018, 1802.04449.

[95] S. Harris, An Introduction to the Theory of the Boltzmann Equation. Dover Publications, 2012.

[96] J. Xu, "Transport approaches for the Description of Intermediate-Energy Heavy-Ion Collisions," Progress in Particle and Nuclear Physics, vol. 106, pp. 312-359, May 2019. arXiv: 1904.00131. 
[97] M. Colonna et al., "Comparison of heavy-ion transport simulations: Mean-field dynamics in a box," Phys. Rev. C, vol. 104, no. 2, p. 024603, 2021, 2106.12287.

[98] J. Weil et al., "Particle production and equilibrium properties within a new hadron transport approach for heavy-ion collisions," Phys. Rev. C, vol. 94, no. 5, p. 054905, $2016,1606.06642$.

[99] J. Cugnon, "Monte Carlo calculation of high-energy heavy-ion interactions," Phys. Rev. C, vol. 22, pp. 1885-1896, 1980.

[100] Y. Yariv and Z. Fraenkel, "Intranuclear cascade calculation of high-energy heavy-ion interactions," Phys. Rev. C, vol. 20, pp. 2227-2243, 1979. [Erratum: Phys.Rev.C 21, 2139-2139 (1980)].

[101] H. Stoecker and W. Greiner, "High-Energy Heavy Ion Collisions: Probing the Equation of State of Highly Excited Hadronic Matter," Phys. Rept., vol. 137, pp. 277-392, 1986.

[102] G. F. Bertsch and S. Das Gupta, "A Guide to microscopic models for intermediateenergy heavy ion collisions," Phys. Rept., vol. 160, pp. 189-233, 1988.

[103] R. Mattiello, H. Sorge, H. Stoecker, and W. Greiner, " $K / \pi$ Ratios in Relativistic Nuclear Collisions: A Signature for the Quark - Gluon Plasma?," Phys. Rev. Lett., vol. 63, pp. 1459-1462, 1989.

[104] S. H. Kahana, D. E. Kahana, Y. Pang, and T. J. Schlagel, "Modeling relativistic heavy ion collisions at the AGS," Ann. Rev. Nucl. Part. Sci., vol. 46, pp. 31-70, 1996.

[105] E. L. Bratkovskaya, M. Bleicher, W. Cassing, M. van Leeuwen, M. Reiter, S. Soff, H. Stoecker, and H. Weber, "Strangeness dynamics in relativistic nucleus nucleus collision," Prog. Part. Nucl. Phys., vol. 53, pp. 225-237, 2004, nucl-th/0312048.

[106] S. A. Bass et al., "Microscopic models for ultrarelativistic heavy ion collisions," Prog. Part. Nucl. Phys., vol. 41, pp. 255-369, 1998, nucl-th/9803035.

[107] W. Cassing and E. L. Bratkovskaya, "Hadronic and electromagnetic probes of hot and dense nuclear matter," Phys. Rept., vol. 308, pp. 65-233, 1999.

[108] W. Cassing and E. L. Bratkovskaya, "Parton transport and hadronization from the dynamical quasiparticle point of view," Phys. Rev. C, vol. 78, p. 034919, 2008, 0808.0022 .

[109] O. Buss, T. Gaitanos, K. Gallmeister, H. van Hees, M. Kaskulov, O. Lalakulich, A. B. Larionov, T. Leitner, J. Weil, and U. Mosel, "Transport-theoretical Description of Nuclear Reactions," Phys. Rept., vol. 512, pp. 1-124, 2012, 1106.1344.

[110] Y. Nara, N. Otuka, A. Ohnishi, K. Niita, and S. Chiba, "Study of relativistic nuclear collisions at AGS energies from $\mathrm{p}+\mathrm{Be}$ to $\mathrm{Au}+\mathrm{Au}$ with hadronic cascade model," Phys. Rev., vol. C61, p. 024901, 2000, nucl-th/9904059.

[111] Z. Xu and C. Greiner, "Thermalization of gluons in ultrarelativistic heavy ion collisions by including three-body interactions in a parton cascade," Phys. Rev. C, vol. 71, p. 064901, 2005, hep-ph/0406278. 
[112] Z.-W. Lin, C. M. Ko, B.-A. Li, B. Zhang, and S. Pal, "A Multi-phase transport model for relativistic heavy ion collisions," Phys. Rev. C, vol. 72, p. 064901, 2005, nucl-th/0411110.

[113] Y.-X. Zhang et al., "Comparison of heavy-ion transport simulations: Collision integral in a box," Phys. Rev., vol. C97, no. 3, p. 034625, 2018, 1711.05950.

[114] P. Huovinen, M. Belkacem, P. J. Ellis, and J. I. Kapusta, "Dileptons and photons from coarse grained microscopic dynamics and hydrodynamics compared to experimental data," Phys. Rev., vol. C66, p. 014903, 2002, nucl-th/0203023.

[115] S. Endres and M. Bleicher, "Dilepton production at SIS energies with the UrQMD model," J. Phys. Conf. Ser., vol. 426, p. 012033, 2013.

[116] S. Endres, H. van Hees, J. Weil, and M. Bleicher, "Coarse-graining approach for dilepton production at energies available at the CERN Super Proton Synchrotron," Phys. Rev., vol. C91, no. 5, p. 054911, 2015, 1412.1965.

[117] S. Endres, H. van Hees, J. Weil, and M. Bleicher, "Dilepton production and reaction dynamics in heavy-ion collisions at SIS energies from coarse-grained transport simulations," Phys. Rev., vol. C92, no. 1, p. 014911, 2015, 1505.06131.

[118] S. Endres, H. van Hees, and M. Bleicher, "Energy, centrality and momentum dependence of dielectron production at collider energies in a coarse-grained transport approach," Phys. Rev., vol. C94, no. 2, p. 024912, 2016, 1604.06415.

[119] S. A. Bass and A. Dumitru, "Dynamics of hot bulk QCD matter: From the quark gluon plasma to hadronic freezeout," Phys. Rev. C, vol. 61, p. 064909, 2000, nuclth/0001033.

[120] D. Teaney, J. Lauret, and E. V. Shuryak, "Flow at the SPS and RHIC as a quark gluon plasma signature," Phys. Rev. Lett., vol. 86, pp. 4783-4786, 2001, nuclth/0011058.

[121] T. Hirano, P. Huovinen, K. Murase, and Y. Nara, "Integrated Dynamical Approach to Relativistic Heavy Ion Collisions," Prog. Part. Nucl. Phys., vol. 70, pp. 108-158, $2013,1204.5814$.

[122] H. Petersen, "Anisotropic flow in transport + hydrodynamics hybrid approaches," J. Phys., vol. G41, no. 12, p. 124005, 2014, 1404.1763.

[123] "IEBE-VISHNU Physics." https://u.osu.edu/vishnu/physics/. [Online; accessed 05-July-2021].

[124] I. A. Karpenko, P. Huovinen, H. Petersen, and M. Bleicher, "Estimation of the shear viscosity at finite net-baryon density from $A+A$ collision data at $\sqrt{s_{\mathrm{NN}}}=7.7-200$ GeV," Phys. Rev. C, vol. 91, no. 6, p. 064901, 2015, 1502.01978.

[125] D. Oliinychenko and H. Petersen, "Forced canonical thermalization in a hadronic transport approach at high density," J. Phys. G, vol. 44, no. 3, p. 034001, 2017, 1609.01087.

[126] P. Danielewicz and G. Bertsch, "Production of deuterons and pions in a transport model of energetic heavy ion reactions," Nucl. Phys. A, vol. 533, pp. 712-748, 1991.

[127] K.-J. Sun, R. Wang, C. M. Ko, Y.-G. Ma, and C. Shen, "Relativistic kinetic approach to light nuclei production in high-energy nuclear collisions," 2021, 2106.12742. 
[128] W. Cassing, "Anti-baryon production in hot and dense nuclear matter," Nucl. Phys. $A$, vol. 700, pp. 618-646, 2002, nucl-th/0105069.

[129] E. Seifert and W. Cassing, "Baryon-antibaryon annihilation and reproduction in relativistic heavy-ion collisions," Phys. Rev. C, vol. 97, no. 2, p. 024913, 2018, 1710.00665 .

[130] E. Seifert and W. Cassing, "Baryon-antibaryon dynamics in relativistic heavy-ion collisions," Phys. Rev. C, vol. 97, no. 4, p. 044907, 2018, 1801.07557.

[131] R. Rapp and E. V. Shuryak, "Resolving the anti-baryon production puzzle in highenergy heavy ion collisions," Phys. Rev. Lett., vol. 86, pp. 2980-2983, 2001, hep$\mathrm{ph} / 0008326$.

[132] L. V. Bravina, E. E. Zabrodin, S. A. Bass, M. Bleicher, M. Brandstetter, S. Soff, H. Stoecker, and W. Greiner, "Local equilibrium in heavy ion collisions: Microscopic analysis of a central cell versus infinite matter," Phys. Rev. C, vol. 62, p. 064906, 2000, nucl-th/0011011.

[133] S. Ryu, J.-F. Paquet, C. Shen, G. Denicol, B. Schenke, S. Jeon, and C. Gale, "Effects of bulk viscosity and hadronic rescattering in heavy ion collisions at energies available at the BNL Relativistic Heavy Ion Collider and at the CERN Large Hadron Collider," Phys. Rev. C, vol. 97, no. 3, p. 034910, 2018, 1704.04216.

[134] A. Lang, H. Babovsky, W. Cassing, U. Mosel, H.-G. Reusch, and K. Weber, "A new treatment of boltzmann-like collision integrals in nuclear kinetic equations," Journal of Computational Physics, vol. 106, no. 2, pp. 391 - 396, 1993.

[135] D. Oliinychenko, L.-G. Pang, H. Elfner, and V. Koch, "Centrality dependence of deuteron production in $\mathrm{Pb}+\mathrm{Pb}$ collisions at $2.76 \mathrm{TeV}$ via hydrodynamics and hadronic afterburner," MDPI Proc., vol. 10, no. 1, p. 6, 2019, 1812.06225.

[136] R. Rapp, J. Wambach, and H. van Hees, "The Chiral Restoration Transition of QCD and Low Mass Dileptons," Landolt-Bornstein, vol. 23, p. 134, 2010, 0901.3289.

[137] V. Koch, "Aspects of chiral symmetry," Int. J. Mod. Phys., vol. E6, pp. 203-250, 1997, nucl-th/9706075.

[138] T. Hatsuda and S. H. Lee, "QCD sum rules for vector mesons in the nuclear medium," Phys. Rev., vol. C46, no. 1, p. R34, 1992.

[139] R. D. Pisarski, "Phenomenology of the Chiral Phase Transition," Phys. Lett., vol. 110B, pp. 155-158, 1982.

[140] G. E. Brown and M. Rho, "Scaling effective Lagrangians in a dense medium," Phys. Rev. Lett., vol. 66, pp. 2720-2723, 1991.

[141] R. Rapp, "The Vector probe in heavy-ion reactions," J. Phys., vol. G31, pp. S217S230, 2005, nucl-th/0409054.

[142] S. Leupold, V. Metag, and U. Mosel, "Hadrons in strongly interacting matter," Int. J. Mod. Phys., vol. E19, pp. 147-224, 2010, 0907.2388.

[143] R. S. Hayano and T. Hatsuda, "Hadron properties in the nuclear medium," Rev. Mod. Phys., vol. 82, p. 2949, 2010, 0812.1702. 
[144] G. E. Brown and M. Rho, "Double decimation and sliding vacua in the nuclear many body system," Phys. Rept., vol. 396, pp. 1-39, 2004, nucl-th/0305089.

[145] R. Rapp and J. Wambach, "Low mass dileptons at the CERN SPS: Evidence for chiral restoration?," Eur. Phys. J., vol. A6, pp. 415-420, 1999, hep-ph/9907502.

[146] C. Jung, F. Rennecke, R.-A. Tripolt, L. von Smekal, and J. Wambach, "In-Medium Spectral Functions of Vector- and Axial-Vector Mesons from the Functional Renormalization Group," Phys. Rev., vol. D95, no. 3, p. 036020, 2017, 1610.08754.

[147] R. Arnaldi et al., "First measurement of the rho spectral function in high-energy nuclear collisions," Phys. Rev. Lett., vol. 96, p. 162302, 2006, nucl-ex/0605007.

[148] S. Damjanovic et al., "First measurement of the rho spectral function in nuclear collisions," Nucl. Phys., vol. A774, pp. 715-718, 2006, nucl-ex/0510044.

[149] G. Agakichiev et al., "Enhanced production of low mass electron pairs in 200-GeV/u S - Au collisions at the CERN SPS," Phys. Rev. Lett., vol. 75, pp. 1272-1275, 1995.

[150] H. van Hees and R. Rapp, "Comprehensive interpretation of thermal dileptons at the SPS," Phys. Rev. Lett., vol. 97, p. 102301, 2006, hep-ph/0603084.

[151] L. Adamczyk et al., "Dielectron Mass Spectra from Au+Au Collisions at $\sqrt{s_{\mathrm{NN}}}=$ $200 \mathrm{GeV}$," Phys. Rev. Lett., vol. 113, no. 2, p. 022301, 2014, 1312.7397. [Addendum: Phys. Rev. Lett.113,no.4,049903(2014)].

[152] A. Adare et al., "Dielectron production in $\mathrm{Au}+\mathrm{Au}$ collisions at $\sqrt{s_{N N}}=200 \mathrm{GeV}$," Phys. Rev., vol. C93, no. 1, p. 014904, 2016, 1509.04667.

[153] G. Agakishiev et al., "Origin of the low-mass electron pair excess in light nucleusnucleus collisions," Phys. Lett., vol. B690, pp. 118-122, 2010, 0910.5875.

[154] G. Agakishiev et al., "Inclusive dielectron production in proton-proton collisions at 2.2 GeV beam energy," Phys. Rev., vol. C85, p. 054005, 2012, 1203.2549.

[155] G. Agakishiev et al., "Inclusive dielectron spectra in $\mathrm{p}+\mathrm{p}$ collisions at $3.5 \mathrm{GeV}$," Eur. Phys. J., vol. A48, p. 64, 2012, 1112.3607.

[156] G. Agakishiev et al., "First measurement of proton-induced low-momentum dielectron radiation off cold nuclear matter," Phys. Lett., vol. B715, pp. 304-309, 2012, 1205.1918 .

[157] G. Agakishiev et al., "Study of dielectron production in C+C collisions at 1-A-GeV," Phys. Lett., vol. B663, pp. 43-48, 2008, 0711.4281.

[158] G. Agakichiev et al., "Dielectron production in C-12+C-12 collisions at 2-AGeV with HADES," Phys. Rev. Lett., vol. 98, p. 052302, 2007, nucl-ex/0608031.

[159] G. Agakishiev et al., "Dielectron production in $\mathrm{Ar}+\mathrm{KCl}$ collisions at $1.76 \mathrm{~A} \mathrm{GeV,"}$ Phys. Rev., vol. C84, p. 014902, 2011, 1103.0876.

[160] R. J. Porter et al., "Dielectron cross-section measurements in nucleus-nucleus reactions at 1-A/GeV," Phys. Rev. Lett., vol. 79, pp. 1229-1232, 1997, nucl-ex/9703001.

[161] J. Adamczewski-Musch et al., "Probing dense baryon-rich matter with virtual photons," Nature Phys., vol. 15, no. 10, pp. 1040-1045, 2019. 
[162] E. L. Bratkovskaya, J. Aichelin, M. Thomere, S. Vogel, and M. Bleicher, "System size and energy dependence of dilepton production in heavy-ion collisions at 1-2 GeV/nucleon energies," Phys. Rev., vol. C87, p. 064907, 2013, 1301.0786.

[163] K. Schmidt, E. Santini, S. Vogel, C. Sturm, M. Bleicher, and H. Stocker, "Production and evolution path of dileptons at energies accessible to the HADES detector," Phys. Rev., vol. C79, p. 064908, 2009, 0811.4073.

[164] C. Hartnack, H. Oeschler, Y. Leifels, E. L. Bratkovskaya, and J. Aichelin, "Strangeness Production close to Threshold in Proton-Nucleus and Heavy-Ion Collisions," Phys. Rept., vol. 510, pp. 119-200, 2012, 1106.2083.

[165] M. Gazdzicki, M. Gorenstein, and P. Seyboth, "Onset of deconfinement in nucleusnucleus collisions: Review for pedestrians and experts," Acta Phys. Polon. B, vol. 42, pp. 307-351, 2011, 1006.1765.

[166] P. Koch, B. Müller, and J. Rafelski, "Strangeness in relativistic heavy ion collisions," Physics Reports, vol. 142, no. 4, pp. 167-262, 1986.

[167] P. Koch, B. Müller, and J. Rafelski, "From strangeness enhancement to quark-gluon plasma discovery," Int. J. Mod. Phys. A, vol. 32, no. 31, p. 1730024, 2017, 1708.08115 .

[168] E. L. Bratkovskaya and W. Cassing, "Dilepton production and off-shell transport dynamics at SIS energies," Nucl. Phys., vol. A807, pp. 214-250, 2008, 0712.0635.

[169] Blume, Christoph, "Open questions in the understanding of strangeness production in hic - experiment perspective," EPJ Web Conf., vol. 171, p. 03001, 2018.

[170] S. Wheaton and J. Cleymans, "THERMUS: A Thermal model package for ROOT," Comput. Phys. Commun., vol. 180, pp. 84-106, 2009, hep-ph/0407174.

[171] A. Andronic, P. Braun-Munzinger, and J. Stachel, "Hadron production in central nucleus-nucleus collisions at chemical freeze-out," Nucl. Phys., vol. A772, pp. 167199, 2006, nucl-th/0511071.

[172] J. Adamczewski-Musch et al., "Deep sub-threshold $\phi$ production in Au+Au collisions," Phys. Lett. B, vol. 778, pp. 403-407, 2018, 1703.08418.

[173] J. Adamczewski-Musch et al., "Sub-threshold production of $\mathrm{K}_{s}^{0}$ mesons and $\Lambda$ hyperons in $\mathrm{Au}+\mathrm{Au}$ collisions at $\sqrt{s_{N N}}=2.4 \mathrm{GeV}$," Phys. Lett., vol. B793, pp. 457-463, 2019, 1812.07304 .

[174] G. Agakishiev et al., "Hyperon production in $\mathrm{Ar}+\mathrm{KCl}$ collisions at $1.76 \mathrm{~A} \mathrm{GeV}$," Eur. Phys. J., vol. A47, p. 21, 2011, 1010.1675.

[175] F. Li, L.-W. Chen, C. M. Ko, and S. H. Lee, "Contributions of hyperon-hyperon scattering to subthreshold cascade production in heavy ion collisions," Phys. Rev., vol. C85, p. 064902, 2012, 1204.1327.

[176] M. Zétényi and G. Wolf, "Influence of anisotropic $\Lambda / \Sigma$ creation on the $\Xi^{-}$mutiplicity in subthreshold proton-nucleus collisions," Phys. Lett., vol. B785, pp. 226-231, 2018, 1803.10573.

[177] J. Steinheimer and M. Bleicher, "Sub-threshold $\phi$ and $\Xi^{-}$production by high mass resonances with UrQMD," J. Phys., vol. G43, no. 1, p. 015104, 2016, 1503.07305. 
[178] P. Braun-Munzinger and B. Dönigus, "Loosely-bound objects produced in nuclear collisions at the LHC," Nucl. Phys. A, vol. 987, pp. 144-201, 2019, 1809.04681.

[179] J. Chen, D. Keane, Y.-G. Ma, A. Tang, and Z. Xu, "Antinuclei in Heavy-Ion Collisions," Phys. Rept., vol. 760, pp. 1-39, 2018, 1808.09619.

[180] D. Oliinychenko, "Overview of light nuclei production in relativistic heavy-ion collisions," Nucl. Phys. A, vol. 1005, p. 121754, 2021, 2003.05476.

[181] K.-J. Sun, L.-W. Chen, C. M. Ko, and Z. Xu, "Probing QCD critical fluctuations from light nuclei production in relativistic heavy-ion collisions," Phys. Lett. $B$, vol. 774 , pp. 103-107, 2017, 1702.07620 .

[182] S. Sombun, K. Tomuang, A. Limphirat, P. Hillmann, C. Herold, J. Steinheimer, Y. Yan, and M. Bleicher, "Deuteron production from phase-space coalescence in the UrQMD approach," Phys. Rev. C, vol. 99, no. 1, p. 014901, 2019, 1805.11509.

[183] D. Oliinychenko, L.-G. Pang, H. Elfner, and V. Koch, "Microscopic study of deuteron production in $\mathrm{PbPb}$ collisions at $\sqrt{s}=2.76 \mathrm{TeV}$ via hydrodynamics and a hadronic afterburner," Phys. Rev. C, vol. 99, no. 4, p. 044907, 2019, 1809.03071.

[184] D. Oliinychenko, C. Shen, and V. Koch, "Deuteron production in AuAu collisions at $\sqrt{s_{N N}}=7-200 \mathrm{GeV}$ via pion catalysis," Phys. Rev. C, vol. 103, no. 3, p. 034913, 2021, 2009.01915.

[185] Y. Oh, Z.-W. Lin, and C. M. Ko, "Deuteron production and elliptic flow in relativistic heavy ion collisions," Phys. Rev. C, vol. 80, p. 064902, 2009, 0910.1977.

[186] R. S. Longacre, "The search for strongly decaying exotic matter," 2013, 1311.3609.

[187] A. Andronic, P. Braun-Munzinger, K. Redlich, and J. Stachel, "The statistical model in Pb-Pb collisions at the LHC," Nucl. Phys. A, vol. 904-905, pp. 535c-538c, 2013, 1210.7724 .

[188] A. Andronic, P. Braun-Munzinger, K. Redlich, and J. Stachel, "Decoding the phase structure of QCD via particle production at high energy," Nature, vol. 561, no. 7723, pp. 321-330, 2018, 1710.09425 .

[189] A. Andronic, P. Braun-Munzinger, B. Friman, P. M. Lo, K. Redlich, and J. Stachel, "The thermal proton yield anomaly in $\mathrm{Pb}-\mathrm{Pb}$ collisions at the LHC and its resolution," Phys. Lett. B, vol. 792, pp. 304-309, 2019, 1808.03102.

[190] J. Stachel, A. Andronic, P. Braun-Munzinger, and K. Redlich, "Confronting LHC data with the statistical hadronization model," J. Phys. Conf. Ser., vol. 509, p. 012019, 2014, 1311.4662 .

[191] D. Everett et al., "Multisystem Bayesian constraints on the transport coefficients of QCD matter," Phys. Rev. C, vol. 103, no. 5, p. 054904, 2021, 2011.01430.

[192] F. Becattini, M. Bleicher, T. Kollegger, M. Mitrovski, T. Schuster, and R. Stock, "Hadronization and Hadronic Freeze-Out in Relativistic Nuclear Collisions," Phys. Rev. C, vol. 85, p. 044921, 2012, 1201.6349.

[193] F. Becattini, E. Grossi, M. Bleicher, J. Steinheimer, and R. Stock, "Centrality dependence of hadronization and chemical freeze-out conditions in heavy ion collisions at $\sqrt{s}_{N N}=2.76 \mathrm{TeV}$," Phys. Rev. C, vol. 90, no. 5, p. 054907, 2014, 1405.0710. 
[194] Y. Pan and S. Pratt, "Baryon annihilation and regeneration in heavy ion collisions," Phys. Rev. C, vol. 89, no. 4, p. 044911, 2014.

[195] V. Steinberg, J. Steinheimer, H. Elfner, and M. Bleicher, "Constraining resonance properties through kaon production in pion-nucleus collisions at low energies," $J$. Phys. G, vol. 48, no. 2, p. 025109, 2021, 1912.09895.

[196] A. Schäfer, J. M. Torres-Rincon, J. Rothermel, N. Ehlert, C. Gale, and H. Elfner, "Benchmarking a nonequilibrium approach to photon emission in relativistic heavyion collisions," Phys. Rev., vol. D99, no. 11, p. 114021, 2019, 1902.07564.

[197] D. Oliinychenko and C. Shen, "Resonance production in $\mathrm{PbPb}$ collisions at $5.02 \mathrm{TeV}$ via hydrodynamics and hadronic afterburner," 2021, 2105.07539.

[198] J. Tindall, J. M. Torres-Rincon, J. B. Rose, and H. Petersen, "Equilibration and freeze-out of an expanding gas in a transport approach in a Friedmann-Robertson-Walker metric," Phys. Lett. B, vol. 770, pp. 532-538, 2017, 1612.06436 .

[199] J. Hammelmann, A. Soto-Ontoso, M. Alvioli, H. Elfner, and M. Strikman, "Influence of the neutron-skin effect on nuclear isobar collisions at energies available at the BNL Relativistic Heavy Ion Collider," Phys. Rev. C, vol. 101, no. 6, p. 061901, 2020, 1908.10231.

[200] J. Mohs, S. Ryu, and H. Elfner, "Particle Production via Strings and Baryon Stopping within a Hadronic Transport Approach," J. Phys. G, vol. 47, no. 6, p. 065101, 2020, 1909.05586.

[201] P. Dorau, J.-B. Rose, D. Pablos, and H. Elfner, "Jet Quenching in the Hadron Gas: An Exploratory Study," Phys. Rev. C, vol. 101, no. 3, p. 035208, 2020, 1910.07027.

[202] J. Mohs, M. Ege, H. Elfner, and M. Mayer, "Collective flow at SIS energies within a hadronic transport approach: Influence of light nuclei formation and equation of state," 2020, 2012.11454.

[203] A. Sorensen and V. Koch, "Phase transitions and critical behavior in hadronic transport with a relativistic density functional equation of state," 2020, 2011.06635.

[204] J. B. Rose, J. M. Torres-Rincon, A. Schäfer, D. R. Oliinychenko, and H. Petersen, "Shear viscosity of a hadron gas and influence of resonance lifetimes on relaxation time," Phys. Rev. C, vol. 97, no. 5, p. 055204, 2018, 1709.03826.

[205] J. B. Rose, J. M. Torres-Rincon, and H. Elfner, "Inclusive and effective bulk viscosities in the hadron gas," J. Phys. G, vol. 48, no. 1, p. 015005, 2020, 2005.03647.

[206] J. Hammelmann, J. M. Torres-Rincon, J.-B. Rose, M. Greif, and H. Elfner, "Electrical conductivity and relaxation via colored noise in a hadronic gas," Phys. Rev. $D$, vol. 99, no. 7 , p. $076015,2019,1810.12527$.

[207] J.-B. Rose, M. Greif, J. Hammelmann, J. A. Fotakis, G. S. Denicol, H. Elfner, and C. Greiner, "Cross-conductivity: novel transport coefficients to constrain the hadronic degrees of freedom of nuclear matter," Phys. Rev. D, vol. 101, no. 11, p. 114028, 2020, 2001.10606. 
[208] A. Ono et al., "Comparison of heavy-ion transport simulations: Collision integral with pions and $\Delta$ resonances in a box," Phys. Rev. C, vol. 100, no. 4, p. 044617, 2019, 1904.02888 .

[209] "SMASH source code." https://github.com/smash-transport/smash. [Online; accessed 2021-June-16].

[210] D. Oliinychenko, V. Steinberg, J. Weil, J. Staudenmaier, M. Kretz, A. Schäfer, H. E. (Petersen), S. Ryu, J. Rothermel, J. Mohs, F. Li, A. Sorensen, D. Mitrovic, L. Pang, J. Hammelmann, A. Goldschmidt, M. Mayer, O. Garcia-Montero, N. Kübler, and Nikita, "smash-transport/smash," June 2021. 10.5281/zenodo.3484711.

[211] D. Oliinychenko, V. Steinberg, J. Weil, M. Kretz, H. E. (Petersen), J. Staudenmaier, S. Ryu, A. Schäfer, J. Rothermel, J. Mohs, F. Li, L. Pang, D. Mitrovic, A. Goldschmidt, L. Geiger, L. Prinz, J.-B. Rose, and J. Hammelmann, "smashtransport/smash: SMASH-1.6," May 2019. 10.5281/zenodo.3485108.

[212] D. Oliinychenko, V. Steinberg, J. Weil, M. Kretz, H. E. (Petersen), J. Staudenmaier, S. Ryu, A. Schäfer, J. Rothermel, F. Li, J. Mohs, L. Pang, A. Goldschmidt, D. Mitrovic, J.-B. Rose, and J. Hammelmann, "smash-transport/smash: SMASH-1.7," Oct. 2019. 10.5281/zenodo.3484712.

[213] D. Oliinychenko, V. Steinberg, J. Weil, J. Staudenmaier, M. Kretz, A. Schäfer, H. E. (Petersen), S. Ryu, J. Rothermel, J. Mohs, F. Li, A. Sorensen, D. Mitrovic, L. Pang, J. Hammelmann, A. Goldschmidt, M. Mayer, O. Garcia-Montero, N. Kübler, and Nikita, "smash-transport/smash: SMASH-2.0," Dec. 2020. 10.5281/zenodo.4336358.

[214] D. M. Manley and E. M. Saleski, "Multichannel resonance parametrization of pi N scattering amplitudes," Phys. Rev. D, vol. 45, pp. 4002-4033, 1992.

[215] J. M. Blatt and V. F. Weisskopf, Theoretical Nuclear Physics. Wiley, 1958.

[216] M. Post, S. Leupold, and U. Mosel, "Hadronic spectral functions in nuclear matter," Nucl. Phys. A, vol. 741, pp. 81-148, 2004, nucl-th/0309085.

[217] J. Xu et al., "Understanding transport simulations of heavy-ion collisions at 100A and $400 \mathrm{~A} \mathrm{MeV}$ : Comparison of heavy-ion transport codes under controlled conditions," Phys. Rev. C, vol. 93, no. 4, p. 044609, 2016, 1603.08149.

[218] J. Walecka, "A theory of highly condensed matter," Annals of Physics, vol. 83, no. 2, pp. 491-529, 1974.

[219] J. I. Kapusta and C. Gale, Finite-temperature field theory: Principles and applications. Cambridge University Press, 2011.

[220] S. Teis, Transporttheoretische Beschreibung von relativistischen Schwerionenkollisionen bei SIS-Energien. PhD thesis, Justus-Liebig-Universität, Gießen, 1996.

[221] T. Kodama, S. B. Duarte, K. C. Chung, R. Donangelo, and R. A. M. S. Nazareth, "Causality and relativistic effects in intranuclear cascade calculations," Phys. Rev. C, vol. 29, pp. 2146-2152, 1984.

[222] T. Hirano and Y. Nara, "Dynamical modeling of high energy heavy ion collisions," PTEP, vol. 2012, p. 01A203, 2012, 1203.4418.

[223] D. Mitrovic, "Lorentz invariance of collision criteria in relativistic heavy-ion collisions." M.Sc. Thesis, Goethe-Universität, Frankfurt am Main, 2020. 
[224] S. Cheng, S. Pratt, P. Csizmadia, Y. Nara, D. Molnar, M. Gyulassy, S. E. Vance, and B. Zhang, "The Effect of finite range interactions in classical transport theory," Phys. Rev. C, vol. 65, p. 024901, 2002, nucl-th/0107001.

[225] "SMASH Analysis Suite." https://github.com/smash-transport/ smash-analysis. [Online; accessed 2021-June-16].

[226] J. Weil, Vector Mesons in Medium in a Transport Approach. PhD thesis, JustusLiebig-Universität, Gießen, 2013.

[227] K. A. Goulianos, "Diffractive Interactions of Hadrons at High-Energies," Phys. Rept., vol. 101, p. 169, 1983.

[228] J. Cugnon, J. Vandermeulen, and D. L'Hote, "Simple parametrization of crosssections for nuclear transport studies up to the GeV range," Nucl. Instrum. Meth. $B$, vol. 111, pp. 215-220, 1996.

[229] V. Dmitriev, O. Sushkov, and C. Gaarde, " $\Delta$ Formation in the ${ }^{1} \mathrm{H}\left({ }^{3} \mathrm{He},{ }^{3} \mathrm{H}\right) \Delta^{++}$ Reaction at Intermediate-energies," Nucl. Phys. A, vol. 459, pp. 503-524, 1986.

[230] G. Graef, J. Steinheimer, F. Li, and M. Bleicher, "Deep sub-threshold $\Xi$ and $\Lambda$ production in nuclear collisions with the UrQMD transport model," Phys. Rev. C, vol. 90, p. 064909, 2014, 1409.7954.

[231] G. Agakishiev et al., "Baryon resonance production and dielectron decays in protonproton collisions at 3.5 GeV," Eur. Phys. J., vol. A50, p. 82, 2014, 1403.3054.

[232] B. Andersson, G. Gustafson, G. Ingelman, and T. Sjostrand, "Parton Fragmentation and String Dynamics," Phys. Rept., vol. 97, pp. 31-145, 1983.

[233] T. Sjöstrand, S. Ask, J. R. Christiansen, R. Corke, N. Desai, P. Ilten, S. Mrenna, S. Prestel, C. O. Rasmussen, and P. Z. Skands, "An introduction to PYTHIA 8.2," Comput. Phys. Commun., vol. 191, pp. 159-177, 2015, 1410.3012.

[234] M. Bleicher et al., "Relativistic hadron hadron collisions in the ultrarelativistic quantum molecular dynamics model," J. Phys. G, vol. 25, pp. 1859-1896, 1999, hep-ph/9909407.

[235] L. Prinz, "Exzentrizitäten im Anfangszustand von Schwerionenkollisionen deformierter Kerne." B.Sc. Thesis, Goethe-Universität, Frankfurt am Main, 2020.

[236] "SMASH-hadron-sampler." https://github.com/smash-transport/smash-hadronsampler.

[237] "SMASH-vHLLE-Hybrid." ～https://github.com/smash-transport/smash-vhllehybrid.

[238] A. Schäfer, O. Garcia-Montero, J. Paquet, H. Elfner, and C. Gale (In preparation).

[239] J. Staudenmaier, "Dileptonenproduktion in hadronischen Transportmodellen." B.Sc. Thesis, 2015.

[240] E. L. Bratkovskaya, W. Cassing, V. P. Konchakovski, and O. Linnyk, "PartonHadron-String Dynamics at Relativistic Collider Energies," Nucl. Phys., vol. A856, pp. 162-182, 2011, 1101.5793.

[241] "SMASH user guide." https://theory.gsi.de/ smash/userguide/current/. [Online; accessed 2021-June-16]. 
[242] G.-Q. Li, C. M. Ko, G. E. Brown, and H. Sorge, "Dilepton production in proton - nucleus and nucleus-nucleus collisions at SPS energies," Nucl. Phys., vol. A611, pp. 539-567, 1996, nucl-th/9611037.

[243] K. A. Olive et al., "Review of Particle Physics," Chin. Phys., vol. C38, p. 090001, 2014.

[244] L. G. Landsberg, "Electromagnetic Decays of Light Mesons," Phys. Rept., vol. 128, pp. 301-376, 1985.

[245] R. Arnaldi et al., "Study of the electromagnetic transition form-factors in eta $\longrightarrow$ mu+ mu- gamma and omega $\longrightarrow$ mu+ mu- pi0 decays with NA60," Phys. Lett., vol. B677, pp. 260-266, 2009, 0902.2547.

[246] E. L. Bratkovskaya and W. Cassing, "Dilepton production from AGS to SPS energies within a relativistic transport approach," Nucl. Phys., vol. A619, pp. 413-446, 1997, nucl-th/9611042.

[247] J. Weil, J. Staudenmaier, and H. Petersen, "Dilepton production with the SMASH model," J. Phys. Conf. Ser., vol. 742, no. 1, p. 012034, 2016, 1604.07028.

[248] M. I. Krivoruchenko and A. Faessler, "Comment on delta radiative and Dalitz decays," Phys. Rev., vol. D65, p. 017502, 2002, nucl-th/0104045.

[249] G. Ramalho, M. T. Pena, J. Weil, H. van Hees, and U. Mosel, "Role of the pion electromagnetic form factor in the $\Delta(1232) \rightarrow \gamma^{*} N$ timelike transition," Phys. Rev., vol. D93, no. 3, p. 033004, 2016, 1512.03764 .

[250] J. Adamczewski-Musch et al., " $\Delta(1232)$ Dalitz decay in proton-proton collisions at $\mathrm{T}=1.25 \mathrm{GeV}$ measured with HADES at GSI," Phys. Rev., vol. C95, no. 6, p. 065205, 2017, 1703.07840.

[251] U. W. Heinz and K. S. Lee, "Dileptons from rho mesons as a fast clock for heavy ion collisions," Nucl. Phys., vol. A544, pp. 503-508, 1992.

[252] G.-Q. Li and C. M. Ko, "Can dileptons reveal the in-medium properties of vector mesons?," Nucl. Phys., vol. A582, pp. 731-748, 1995, nucl-th/9407016.

[253] T. Galatyuk, P. M. Hohler, R. Rapp, F. Seck, and J. Stroth, "Thermal Dileptons from Coarse-Grained Transport as Fireball Probes at SIS Energies," Eur. Phys. J., vol. A52, no. 5, p. 131, 2016, 1512.08688.

[254] L. D. McLerran and T. Toimela, "Photon and dilepton emission from the quarkgluon plasma: Some general considerations," Phys. Rev. D, vol. 31, pp. 545-563, Feb 1985 .

[255] R. Rapp, "Signatures of thermal dilepton radiation at RHIC," Phys. Rev., vol. C63, p. 054907, 2001, hep-ph/0010101.

[256] M. Tanabashi et al., "Review of Particle Physics," Phys. Rev. D, vol. 98, no. 3, p. 030001, 2018.

[257] F. Scozzi, "Resonance production and decay in pion induced collisions with HADES," EPJ Web Conf., vol. 137, p. 05023, 2017.

[258] C. Franco, "Dilepton Results from HADES Using Au+Au Data at 1.23 AGeV," JPS Conf. Proc., vol. 13, p. 020003, 2017. 
[259] H. Calen et al., "Measurement of the quasifree $\mathrm{p}+\mathrm{n} \rightarrow \mathrm{d}+$ eta reaction near threshold," Phys. Rev. Lett., vol. 79, pp. 2642-2645, 1997.

[260] E. C. et al., "Measurement of the pp $\rightarrow$ pp $\eta$ total cross-section between 1.265 and 1.50 gev," Physics Letters B, vol. 322, no. 3, pp. 270 - 274, 1994.

[261] H. E. Schopper, Landolt-Börnstein, New Series, Vol. I/12. Springer, 1988.

[262] V. Flaminio, W. G. Moorhead, D. R. O. Morrison, and N. Rivoire, "Compilation of Cross-sections. 3. $P$ and $\bar{P}$ Induced Reactions," 1984.

[263] H. B. O'Connell, B. C. Pearce, A. W. Thomas, and A. G. Williams, " $\rho-\omega$ mixing, vector meson dominance and the pion form-factor," Prog. Part. Nucl. Phys., vol. 39, pp. 201-252, 1997, hep-ph/9501251.

[264] H. Calen et al., "Measurement of the quasifree $\mathrm{p}+\mathrm{n}->\mathrm{p}+\mathrm{n}+$ eta reaction near threshold," Phys. Rev., vol. C58, pp. 2667-2670, 1998.

[265] S. Teis, W. Cassing, M. Effenberger, A. Hombach, U. Mosel, and G. Wolf, "Pion production in heavy ion collisions at SIS energies," Z. Phys., vol. A356, pp. 421-435, 1997, nucl-th/9609009.

[266] "Adapted HADES filter." http://www.github.com/janusw/HAFT. [Online; accessed 2021-June-17].

[267] M. Abdel-Bary et al., "Study of spectator tagging in the reaction np $->$ pp pi with a deuteron beam," Eur. Phys. J., vol. A29, pp. 353-361, 2006.

[268] M. Lacombe, B. Loiseau, R. Vinh Mau, J. Cote, P. Pires, and R. de Tourreil, "Parametrization of the deuteron wave function of the Paris n-n potential," Phys. Lett., vol. 101B, pp. 139-140, 1981.

[269] J. Adamczewski-Musch et al., "Analysis of the exclusive final state npe ${ }^{+} \mathrm{e}^{-}$in quasifree np reaction," Eur. Phys. J., vol. A53, no. 7, p. 149, 2017, 1703.08575.

[270] R. Shyam and U. Mosel, "Dilepton production in proton-proton and quasi-free proton-neutron reactions at $1.25 \mathrm{GeV}$," Phys. Rev., vol. C82, p. 062201, 2010, 1006.3873.

[271] M. Bashkanov and H. Clement, "On a Possible Explanation of the DLS Puzzle," Eur. Phys. J., vol. A50, p. 107, 2014, 1312.2810.

[272] B. V. Martemyanov, M. I. Krivoruchenko, and A. Faessler, "Dilepton production in pp and np collisions at 1.25 GeV," Phys. Rev., vol. C84, p. 047601, 2011, 1108.4265.

[273] B. Friman and H. J. Pirner, "P wave polarization of the rho meson and the dilepton spectrum in dense matter," Nucl. Phys., vol. A617, pp. 496-509, 1997, nuclth/9701016.

[274] W. Peters, M. Post, H. Lenske, S. Leupold, and U. Mosel, "The Spectral function of the rho meson in nuclear matter," Nucl. Phys., vol. A632, pp. 109-127, 1998, nucl-th/9708004.

[275] R. Rapp, M. Urban, M. Buballa, and J. Wambach, "A Microscopic calculation of photoabsorption cross-sections on protons and nuclei," Phys. Lett., vol. B417, pp. 16, 1998, nucl-th/9709008. 
[276] R. Rapp and J. Wambach, "Chiral symmetry restoration and dileptons in relativistic heavy ion collisions," Adv. Nucl. Phys., vol. 25, p. 1, 2000, hep-ph/9909229.

[277] M. Zetenyi and G. Wolf, "Baryonic contributions to the dilepton spectrum of nucleon-nucleon collisions," Phys. Rev., vol. C67, p. 044002, 2003, nucl-th/0103062.

[278] G. Agakishiev et al., "Measurement of charged pions in C-12 + C-12 collisions at 1AGeV and 2A-GeV with HADES," Eur. Phys. J., vol. A40, pp. 45-59, 2009, 0902.4377.

[279] F. Seck, T. Galatyuk, R. Rapp, and J. Stroth, "Probing the Fireball at SIS-18 Energies with Thermal Dilepton Radiadtion," Acta Phys. Polon. Supp., vol. 10, pp. $717-721,2017$.

[280] J. Adamczewski-Musch et al., "Probing dense baryon-rich matter with virtual photons," Nature Phys., vol. 15, no. 10, pp. 1040-1045, 2019.

[281] J. T. Balewski et al., "Total cross-section of the reaction p p $\rightarrow$ p K+ Lambda close to threshold," Phys. Lett. B, vol. 420, pp. 211-216, 1998, nucl-ex/9803003.

[282] S. Sewerin et al., "Comparison of Lambda and Sigma**0 threshold production in proton proton collisions," Phys. Rev. Lett., vol. 83, pp. 682-685, 1999, nucl-ex/9811004.

[283] P. Kowina et al., "Energy dependence of the Lambda / Sigma0 production crosssection ratio in p p interactions," Eur. Phys. J. A, vol. 22, pp. 293-299, 2004, nucl-ex/0402008.

[284] S. Abd El-Samad et al., "Influence of $\mathrm{N}^{*}$-resonances on hyperon production in the channel pp- $>\mathrm{K}+$ Lambda p at 2.95, 3.20 and $3.30 \mathrm{GeV} / \mathrm{c}$ beam momentum," Phys. Lett. B, vol. 688, pp. 142-149, 2010, 1003.0603.

[285] M. Abdel-Bary et al., "Production of Lambda and Sigma0 hyperons in proton-proton collisions," Eur. Phys. J. A, vol. 46, pp. 27-44, 2010, 1008.4287. [Erratum: Eur. Phys. J.A46,435(2010)].

[286] R. Bilger et al., "Strangeness production in the reaction $\mathrm{pp}->\mathrm{K}+$ Lambda $\mathrm{p}$ in the threshold region," Phys. Lett. B, vol. 420, pp. 217-224, 1998.

[287] S. Abdel-Samad et al., "Hyperon production in the channel p p $\rightarrow \mathrm{K}+$ Lambda $\mathrm{p}$ near the reaction threshold," Phys. Lett. B, vol. 632, pp. 27-34, 2006.

[288] Yu. Valdau et al., "The pp $->\mathrm{K}+\mathrm{n}$ Sigma+ reaction near threshold," Phys. Lett. $B$, vol. 652, pp. 245-249, 2007, nucl-ex/0703044.

[289] Yu. Valdau et al., "The energy dependence of the pp- $>\mathrm{K}+\mathrm{n}$ Sigma+ reaction close to threshold," Phys. Rev. C, vol. 81, p. 045208, 2010, 1002.3459.

[290] A. Baldini, "Numerical data and functional relationships in science and technology. grp. 1: Nuclear and particle physics. vol. 12: Total cross-sections for reactions of high-energy particles (including elastic, topological, inclusive and exclusive reactions).," Landolt-Börnstein. New Series, 1/12B, 1988.

[291] J. E. Bernhard, J. S. Moreland, S. A. Bass, J. Liu, and U. Heinz, "Applying Bayesian parameter estimation to relativistic heavy-ion collisions: simultaneous characterization of the initial state and quark-gluon plasma medium," Phys. Rev. C, vol. 94, no. 2, p. $024907,2016,1605.03954$. 
[292] J. Adamczewski-Musch et al., "Deep sub-threshold $\phi$ production in Au+Au collisions," Phys. Lett. B, vol. 778, pp. 403-407, 2018, 1703.08418.

[293] S. Petschauer, J. Haidenbauer, N. Kaiser, U.-G. Meißner, and W. Weise, "Hyperons in nuclear matter from SU(3) chiral effective field theory," Eur. Phys. J. A, vol. 52, no. 1 , p. $15,2016,1507.08808$.

[294] D. Miskowiec, "Nuclear overlap calculation." http://web-docs.gsi.de/ misko/ overlap/. Accessed: 2020-03-24.

[295] J. Steinheimer and M. Bleicher, "Sub-threshold strangeness and charm production in UrQMD," J. Phys. Conf. Ser., vol. 779, no. 1, p. 012017, 2017.

[296] G. Agakishiev et al., "Subthreshold $\Xi^{-}$Production in Collisions of $\mathrm{p}(3.5 \mathrm{GeV})+\mathrm{Nb}$," Phys. Rev. Lett., vol. 114, no. 21, p. 212301, 2015, 1501.03894.

[297] K. G. M. Cercignani, Carlo, The relativistic Boltzmann equation: theory and applications. Birkhäuser Basel, 2002.

[298] E. Byckling and K. Kajantie, Particle Kinematics: (Chapters I-VI, X). University of Jyvaskyla, 1971.

[299] S. Bauberger, F. A. Berends, M. Bohm, and M. Buza, "Analytical and numerical methods for massive two loop selfenergy diagrams," Nucl. Phys. B, vol. 434, pp. 383407, 1995, hep-ph/9409388.

[300] A. I. Davydychev and R. Delbourgo, "Explicitly symmetrical treatment of three body phase space," J. Phys. A, vol. 37, pp. 4871-4886, 2004, hep-th/0311075.

[301] "The Giessen BUU Project." https://gibuu.hepforge.org/. [Online; accessed 30-June-2021].

[302] J. Torres-Rincon. personal communication.

[303] P. Koch, B. Muller, and J. Rafelski, "Strangeness in Relativistic Heavy Ion Collisions," Phys. Rept., vol. 142, pp. 167-262, 1986.

[304] C. B. Dover, T. Gutsche, M. Maruyama, and A. Faessler, "The Physics of nucleon - anti-nucleon annihilation," Prog. Part. Nucl. Phys., vol. 29, pp. 87-174, 1992.

[305] T. Neidig, "Production of light nuclei in ultra-relativistic heavy-ion collisions via a network of rate equations." M.Sc. Thesis, Goethe-Universität, Frankfurt am Main, 2020.

[306] J. Aichelin, E. Bratkovskaya, A. Le Fèvre, V. Kireyeu, V. Kolesnikov, Y. Leifels, V. Voronyuk, and G. Coci, "Parton-hadron-quantum-molecular dynamics: A novel microscopic $n$-body transport approach for heavy-ion collisions, dynamical cluster formation, and hypernuclei production," Phys. Rev. C, vol. 101, no. 4, p. 044905, 2020, 1907.03860.

[307] J.-F. Paquet, C. Shen, G. S. Denicol, M. Luzum, B. Schenke, S. Jeon, and C. Gale, "Production of photons in relativistic heavy-ion collisions," Phys. Rev., vol. C93, no. 4, p. 044906, 2016, 1509.06738.

[308] G. S. Denicol, C. Gale, S. Jeon, A. Monnai, B. Schenke, and C. Shen, "Net baryon diffusion in fluid dynamic simulations of relativistic heavy-ion collisions," Phys. Rev. $C$, vol. 98 , no. 3 , p. $034916,2018,1804.10557$. 
[309] C. Shen and S. Alzhrani, "Collision-geometry-based 3D initial condition for relativistic heavy-ion collisions," Phys. Rev. C, vol. 102, no. 1, p. 014909, 2020, 2003.05852.

[310] A. Monnai, B. Schenke, and C. Shen, "Equation of state at finite densities for QCD matter in nuclear collisions," Phys. Rev. C, vol. 100, no. 2, p. 024907, 2019, 1902.05095 .

[311] L. Adamczyk et al., "Bulk Properties of the Medium Produced in Relativistic HeavyIon Collisions from the Beam Energy Scan Program," Phys. Rev. C, vol. 96, no. 4, p. $044904,2017,1701.07065$.

[312] J. Adam et al., "Beam energy dependence of (anti-)deuteron production in $\mathrm{Au}+\mathrm{Au}$ collisions at the BNL Relativistic Heavy Ion Collider," Phys. Rev. C, vol. 99, no. 6, p. 064905, 2019, 1903.11778.

[313] T. Anticic et al., "Production of deuterium, tritium, and $\mathrm{He} 3$ in central $\mathrm{Pb}+\mathrm{Pb}$ collisions at 20A,30A,40A,80A , and $158 \mathrm{~A} \mathrm{GeV}$ at the CERN Super Proton Synchrotron," Phys. Rev. C, vol. 94, no. 4, p. 044906, 2016, 1606.04234.

[314] L. Adamczyk et al., "Measurement of elliptic flow of light nuclei at $\sqrt{s_{N N}}=200$, 62.4, 39, 27, 19.6, 11.5, and 7.7 GeV at the BNL Relativistic Heavy Ion Collider," Phys. Rev. C, vol. 94, no. 3, p. 034908, 2016, 1601.07052.

[315] J. Steinheimer, S. Schramm, and H. Stocker, "An Effective chiral Hadron-Quark Equation of State," J. Phys. G, vol. 38, p. 035001, 2011, 1009.5239.

[316] P. Huovinen and H. Petersen, "Particlization in hybrid models," Eur. Phys. J. A, vol. 48, p. 171, 2012, 1206.3371.

[317] F. Becattini, J. Cleymans, A. Keranen, E. Suhonen, and K. Redlich, "Features of particle multiplicities and strangeness production in central heavy ion collisions between 1.7A-GeV/c and 158A-GeV/c," Phys. Rev. C, vol. 64, p. 024901, 2001, hep-ph/0002267.

[318] S. Acharya et al., "Production of charged pions, kaons, and (anti-)protons in $\mathrm{Pb}-\mathrm{Pb}$ and inelastic $p p$ collisions at $\sqrt{s_{N N}}=5.02 \mathrm{TeV}$," Phys. Rev. C, vol. 101, no. 4, p. $044907,2020,1910.07678$.

[319] S. S. Adler et al., "Identified charged particle spectra and yields in Au+Au collisions at $\mathrm{S}(\mathrm{NN})^{* *} 1 / 2=200-\mathrm{GeV}$," Phys. Rev. C, vol. 69, p. 034909, 2004, nucl-ex/0307022.

[320] I. A. Karpenko, Y. M. Sinyukov, and K. Werner, "Uniform description of bulk observables in the hydrokinetic model of $A+A$ collisions at the BNL Relativistic Heavy Ion Collider and the CERN Large Hadron Collider," Phys. Rev. C, vol. 87, no. 2, p. $024914,2013,1204.5351$.

[321] G. Balassa and G. Wolf, "A statistical model to calculate inclusive hadronic crosssections," Eur. Phys. J. A, vol. 56, no. 6, p. 174, 2020, 2102.12442.

[322] "Open Science Wikipedia." https://en.wikipedia.org/wiki/Open_science. [Online; accessed 2021-June-16].

[323] "SMASH documentation." http://theory.gsi.de/ smash/doc/current/. [Online; accessed 2021-June-16]. 


\section{Appendix A}

\section{Numerical realization and implementation details of the stochastic criterion}

This appendix lists relevant technical details on numerical realization and implementation of the stochastic criterion in the transport approach SMASH, which form the basis for the physical results discussed in the main parts of this work.

\section{A.1 Open Software and setup on Github}

All results of the transport approach SMASH in this thesis are easily reproducible and maximally transparent since the source code is publicly available [209]. SMASH was developed as an open source code under the GPL-3.0 license from the beginning and was made publicly available at the end of 2018 .

Considerable effort is made to follow best practices of Open Source development and to adhere to the overall principle of Open Science [322]. This entails that the program is versioned consistently and clearly documented, with all recent versions being citable by their own DOI [210]. This is relevant for this work, since different results are only reproducible with certain program versions. All major versions are thoroughly checked for the stability of a basal set of physical results [225]. All changes to the code are required to pass unit tests and automatic checks ensure that documentation and formatting guidelines are satisfied. Furthermore, the program is presented in a way that external researchers can easily contribute, which simultaneously ensures accessibility and easy-ofuse for researchers, who (just) want to employ the code to reproduce results, compare to experimental data or use it for their own studies. For all this, the code is documented extensively [241, 323].

Even though Open Software is not common in the heavy-ion field of research, the contributors to the SMASH approach believe that it is a good scientific practice and ensures the reproducibility and traceability of the results and in term lends credibly to results. Furthermore, the project is publicly funded and, therefore, should be available to the public.

Github is chosen as the platform to make this availability possible. As it is the most 


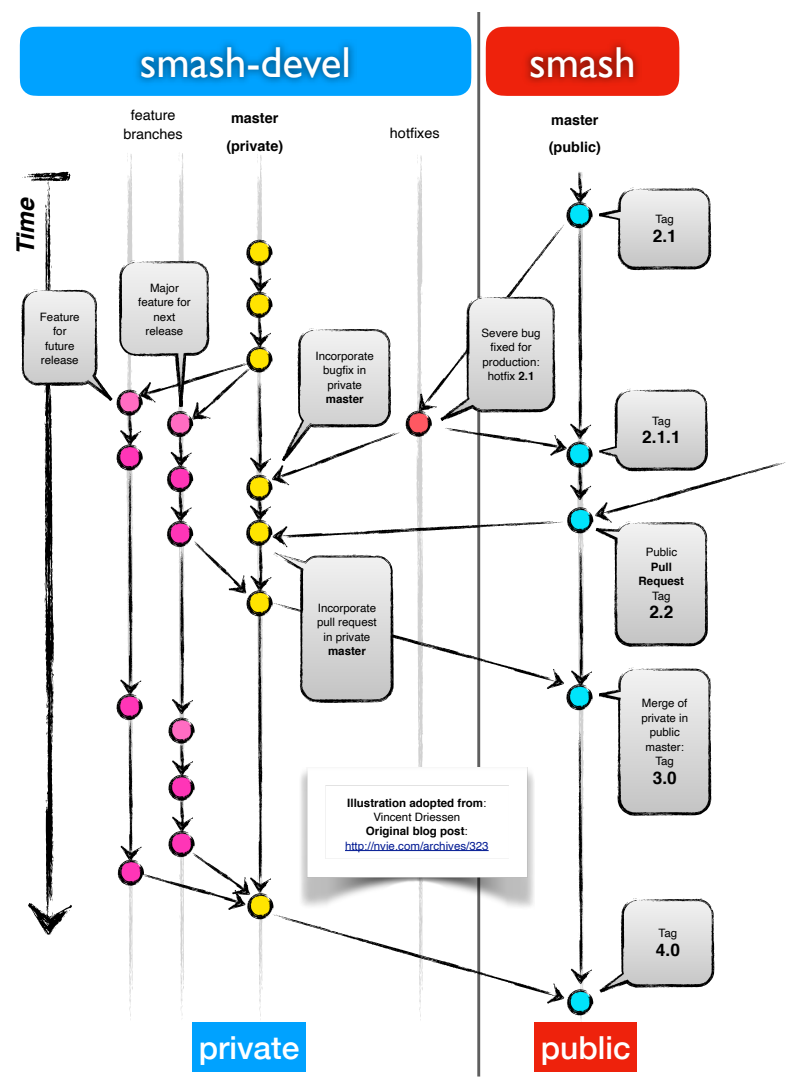

Figure A.1: Two repository setup and workflow for the SMASH source code.

popular platform for open source codes, it allows to organize large software efforts easily. It enables internal and external collaboration by providing functionality like issue tracking, pull requests and a wiki. To prevent all beginning students from developing publicly and protect new, unpublished work if necessary, two repositories, a public and a private one, are employed. Both are regularly synced when a new version is released (tagged). The procedure of this syncing is displayed in Figure A.1. New developments are usually done on feature branches in the private repositories. They are only merged after a review process performed by one of the other contributors and by an automatic continuous integration routine that runs the unit tests and ensures complete documentation and correct formatting. A new version is released when both master branches are merged. This either occurs when changes to the public version (e.g. by an external contribution) are made or on a bi-annual schedule.

\section{A.2 Numerical realization of the stochastic criterion}

The stochastic criterion ${ }^{1}$ in the SMASH approach builds on the existing realization of the geometric criterion. Since the only essential difference between both is how it is decided whether and when two incoming particles scatter, the surrounding routine is mostly identical for both criteria. The main difference concerns the role of the grid, which

\footnotetext{
${ }^{1}$ In this section, the application of the stochastic criterion for 2-body reactions is discussed, which matches the applicability of the geometric criterion. The extension to multi-particle reaction is discussed in the following section.
} 
is an essential calculation input and not a mere optimization for the stochastic criterion (see subsection below).

The general routine for two particles to scatter is as follows. First all possible two particle pairs within a grid cell are paired (find_actions_in_cell ()$\left.^{2}\right)$. Those pairs of incoming particles are then checked for a (two) particle collision (check_collision_two_part(), formerly check_collision()). For this potential scattering (ScatterAction), the total cross-section is calculated as the sum of the partial cross-sections of all possible processes between the incoming particles. The total cross-section is then scaled when the incoming particles are not fully formed. In the case of the stochastic criterion, the collision probability is then calculated using the total cross-section according to Eq. 4.25:

$$
P_{2 \rightarrow m}=\frac{\Delta t}{\Delta^{3} x} v_{r e l} \sigma_{2 \rightarrow m}
$$

As shown in Section 4.1, the equation is valid for all 2-body collisions, which includes elastic or inelastic 2-to-2, 2-to-1 resonance formations and 2-to-m string fragmentations. The relative velocity $\left(v_{r e l}\right)$ and total cross-section $\left(\sigma_{2 \rightarrow m}\right)$ are physical observables between the scattering partners. Whereas $\Delta t$ and $\Delta^{3} x$ are numerical calculation parameters. $\Delta t$ is the timestep size of the calculation, which is provided as an input of the calculation in the configuration file (Delta_Time). The grid cell volume determines $\Delta^{3} x$. All quantities are considered in the calculation frame.

The calculated collision probability is used for a Monte Carlo decision, whether a collision between both particles is performed. A random number between 0 and 1 is generated and the collision is accepted when the random number is equal or below the collision probability. The collision probability is limited to 1 . If it exceeds 1 depending on the configuration, an error (default) or a warning is emitted by SMASH. Which of the different processes contributing to the total cross-section is performed is also randomly decided based on the fraction $\sigma_{\text {partial }} / \sigma_{\text {total }}$ of the process. Contrary to the geometric criterion, scatterings are not rejected because they are farther away than the maximal transverse distance, which stems from the geometric interpretation from the maximal cross-section. The cells are anyway required to be small. Also, reactions of particles that have just reacted are allowed to react again directly. This avoids any biases in the stochastic treatment of the evolution (i.e. the underlying Markov chain). This is also found in calculations, where immediate back-reactions are needed to fully reproduce the correct scattering rate (cf. Figure 5.1 and Figure 5.14). But, allowing immediate back-reactions also leads to some artificial increase of scatterings for sparsely filled, small cells (as discussed in Section 5.1.5). The time when particles scatter is distributed randomly within the given timestep. As the position of newly produced particles in inelastic scatterings, the position of one randomly determined incoming particle is chosen. This placing avoids density artifacts, in particular when calculating with periodic boundary conditions. The final momenta are distributed as usual by SMASH mostly isotropically (see Section 2.2.3). The sampling of the outgoing momenta for the 5-body final state needed to be added in addition (see Figure A.3 and related discussion below).

Note that the stochastic collision treatment is introduced over multiple versions of SMASH. The stochastic criterion for binary reactions is introduced with version SMASH-1.7 [212]. Three-body reactions are available since SMASH-2.0 [213] and the 5-to-2 action will be

\footnotetext{
${ }^{2}$ Where fitting, function, class or config parameter names from the SMASH source code [209] are provided in monospace lettering in the following.
} 
part of the next minor tagged version of SMASH. For the latter, all results include the specific commit hash needed to reproduce them (instead of the not yet available tagged version).

\section{A.2.1 Grid}

The role of the grid is different from the geometric criterion. In the geometric case, the grid is a mere optimization of the particle number $(N)$ scaling [98]. Collisions are searched within a grid cell and with particles in neighboring cells (nearest neighbor search). Thereby, not every particle pair is considered, leading to scaling the computing time with less than $N^{2}$. Particle pairs that (within the current timestep $\Delta t$ ) cannot get closer than the transverse distance $\left(d_{T, \max }\right)$ determined by the maximum cross-section $\left(\sigma_{\max }\right)$ are neglected by the geometric collision search. Therefore, the minimal grid cell length in this case is given by $l_{\min }=\sqrt{\left((2 \Delta t)^{2}+d_{T, \max }^{2}\right.}$ with $d_{T, \max }=\sqrt{\frac{\sigma_{\max }}{\pi N_{\text {test }}}}$. This ensures that all particles are in adjacent cells that should be considered for a scattering i.e. are not too far apart for the geometric interpretation of the cross-section. The maximum cross-section is $200 \mathrm{mb}$ by default, which translates to $l_{\min } \approx 2.5 \mathrm{fm}$. Note that $d_{T, \max }$ and consequently $l_{\min }$ is scaled when employing test particles. In order to vary the grid size for the stochastic criterion (like in Section 5.1.5), the maximum cross-section $\sigma_{\max }$ has to be changed in the config file (parameter: Maximum_Cross_Section) to the value corresponding to the the desired $l_{\min }$. Note that the value specified for $\sigma_{\max }$ has to be multiplied by $N_{\text {test }}$ to prevent a grid size scaling with the test particle number when fixing the grid size manually ${ }^{3}$.

The general structure of the grid can be directly applied for the stochastic criterion with only minor changes necessary. It is, however, not an optimization but an essential part of the calculation itself. The stability of calculation results under changes for the grid (cell volume) is discussed in detail in Section 5.1.5 for this reason. For the stochastic criterion, only particles within the same cell are checked for collisions and no neighbor search occurs. That is, since the collision probability is defined only for a certain subvolume $\Delta^{3} x$, which in SMASH equals a grid cell. Particularly useful is the dynamic grid setup, which spans from the minimum to the maximum position of all particles along the three axis. The grid, therefore, dynamically expands with the medium. Only in the case of a box calculation, the grid is static and the size equals the size of the box. The grid construction was adapted for this work always to have equal sized cell lengths for a given axis. In this way, all cells have the same volume $\Delta^{3} x$ for a given timestep, which is needed for the collision probability calculation. The length of the grid is divided by $l_{\min }$ to obtain the number of cells along an axis. The cell lengths are scaled up until they match the length of the grid, which is also why only a minimum cell length is given. Note that should the grid have zero length along one axis and the grid cell volume would be 0 , the collision probability would diverge. Therefore, in this case, collisions are rejected since no probability is defined. An example of this extreme case are elementary collision systems like proton-proton. The stochastic criterion is not applicable for such cases. However, for such dilute systems, a stochastic interpretation of the scattering process is in any case questionable. Furthermore, for all presented results, the dynamic grid setup is changed ${ }^{4}$ to not limit the number of cells per dimension to $\sqrt[3]{N}$. In this way, the geometric criterion is usually optimized for the dilute limit (one cell per particle), which is most likely to

\footnotetext{
${ }^{3} \mathrm{~A}$ timely change to SMASH to include a specific (minimal) gird length configuration parameter that is not scaled by the test particle number is planned.

${ }^{4}$ A permanent modification to SMASH to ignore the limitation of the number of cells for the stochastic criterion by default is planned.
} 
be reached in afterburner calculations. Instead of creating more grid cells as the system expands, the cell sizes are grown. In case of the geometric criterion, this does not change the collision parings. But, since the stochastic criterion is dependent on the cell size itself, they ought to be fixed, even though this requires a larger number of cells than particles of which many might be empty.

\section{A.3 Code design of the ScatterActionMulti class}

While the stochastic criterion only requires minor changes $^{5}$, realizing multi-particle reactions in the SMASH transport approach is a larger effort, since most of the available structure expects that only two particles scatter. Nevertheless, being built as a modular approach, adding the new functionality of more than two-particle scattering can be realized without large changes to the existing codebase. The main additions and changes are explained in the following. At the end of the section, more practical explanations on employing multi-particles for calculations are given.

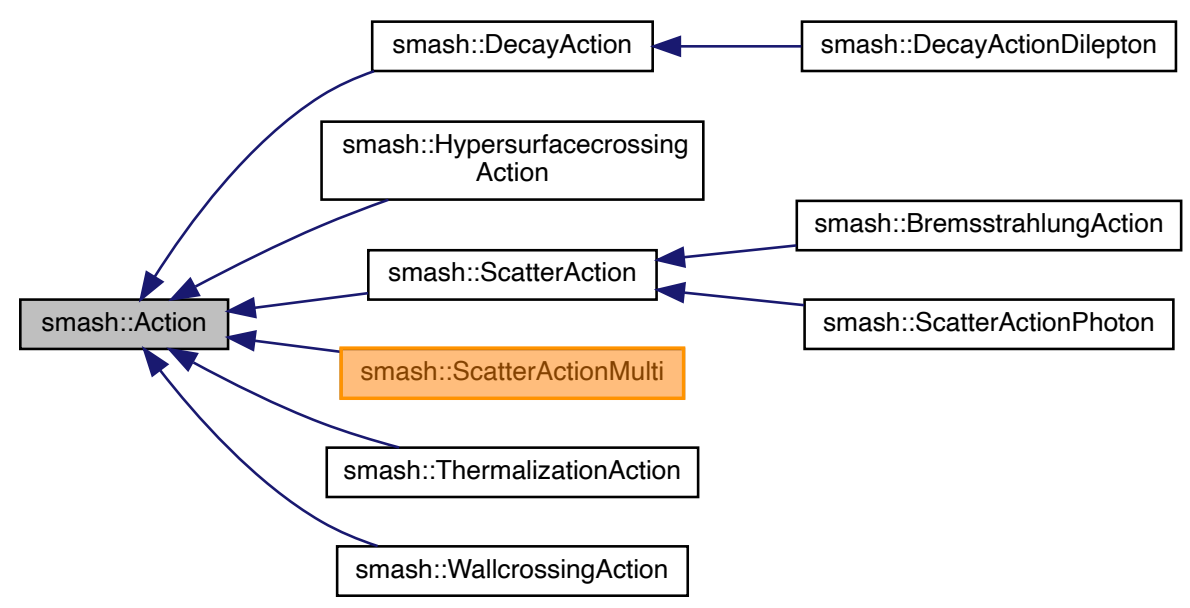

Figure A.2: Action class hierarchy in SMASH. New ScatterActionMulti class highlighted.

The only major change to existing functionality concerns the pairing of particles in a given grid cell. With the addition of multi-particle reactions, multi-particle pairings are needed to be checked for reactions as well. Therefore, the new check_collision_multi_part() is called with lists of all possible 3 or 5 incoming particle parings (in find_actions_in_ cell()), but only when the corresponding multi-particle reaction is included in the calculation. The decision of whether a multi-particle reaction is accepted is analog to the two-body collision with the collision probability being used of a Monte Carlo decision. The only difference is that not the cross-section (which is ill-defined for multiple particles) being scaled by the number of test particles, but the collision probability itself (by $N_{\text {test }}^{-(n-1)}$ ), where $n$ is the number of incoming particles). The collision time is distributed

\footnotetext{
${ }^{5}$ mainly to check_collision() in the ScatterActionsFinder class
} 
randomly within the timestep and the position of the outgoing particles is randomly chosen as one of the incoming particle positions, like for two-body stochastic collisions. The Collisions_Within_Nucleus flag that determines whether to allow the first collisions within the same nucleus works in the same fashion as for other scatter actions.

All routines related to multi-particle actions are part of a new Action class: the Scatter ActionMulti. The class is like the other Action classes derived from the Action base class (see FigureA.2). It is necessary to add a new Action class besides the existing ScatterAction class, since the ScatterAction class requires exactly two incoming particles throughout almost all of its functionality. The new ScatterActionMulti takes an arbitrary long list of incoming particles. A ScatterActionMulti is created for all particle pairings within each cell. The main method of the class is add_possible_reactions(), which adds all possible scatterings for the incoming particles as channels to created action object, if there are any. The already calculated collision probabilities (see Section 4.3 for the equations) are stored as the weights of the processes. As such, the class holds the calculations for all implemented multi-particle collision probabilities (probability_three_to_ one(), probability_three_to_two() and probability_five_to_two()) as well as the needed 3- and 5-body phase space integrations (calculate_I3() and parametrization phi5_pions ()). A difference compared to the (two-body) ScatterAction is that the total (partial) weight of the multi-particle action is not the total (partial) cross-section, but the total (partial) collision probability. If a multi-particle reaction is accepted, the final state is generated like for other action classes (in generate_final_state()) with the appropriate (already existing) sampling of the outgoing phase space. If more than one reaction is possible between the incoming particles, the reaction is chosen according to the partial weight, which is the collision probability of the specific reaction. A full code documentation of the ScatterActionMulti class is found as part of the general code documentation under [323].

In addition to implementing the multi-particle reactions themselves, also their reverse counterparts need to be treated. For the 3 -to- 1 reactions, the reverse reaction $(1 \rightarrow 3$ decay) is already possible by changing the decay modes input file. For the 2-to-3 and 2 -to-5 reactions, the CrossSections class is changed. The 2-to-5 reaction $(N \bar{N} \rightarrow$ $\left.\pi^{+} \pi^{-} \pi^{+} \pi^{-} \pi^{0}\right)$ mirrors the treatment via resonances $\left(N \bar{N} \rightarrow h_{1} \rho\right)$. The 2 -to-3 deuteron reaction also uses the same cross-section parameters as the treatment involving the $d^{\prime}$. The final state, however, is for both classes directly i.e. in one step generated. Here, in addition the sampling of the 5-body phase space had to be added and tested (sample_5body_phase space()). As seen in Figure A.3, the sampling produced an isotropic distribution. The generation of the final state by the ScatterAction class is adapted to account for the new 2-to-3 and 2-to-5 reactions.

To distinguish the different processes, new ProcessTypes were included for all directions: TwoToThree, TwoToFive, MultiParticleThreeMesonsToOne, MultiParticleThreeTo Two, MultiParticleFiveToTwo. Two unit tests for the mesonic 3-to-1 and 3-to-2 deuteron processes are added to check that the correct number of reaction channels with the correct process types are found when the correct initial state is given as the input to the relevant functions.

\section{A.3.1 Using multi-particle reactions in SMASH}

Multi-particle reaction, as well as the stochastic criterion, can be enabled in the configuration file (config.yaml). The stochastic criterion is necessary to be employed when 

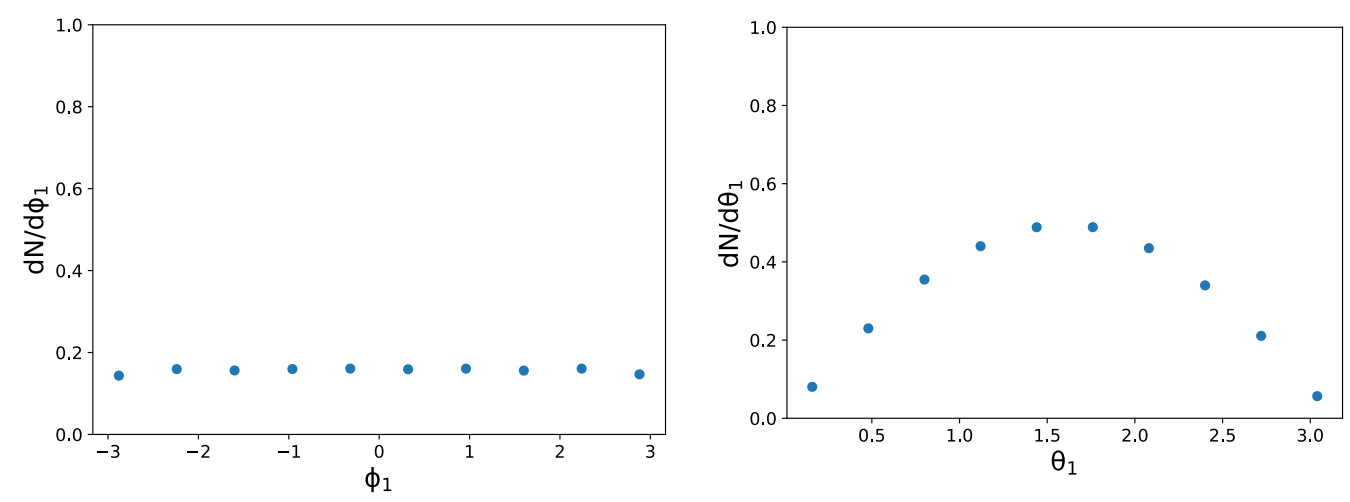

Figure A.3: Test of new isotropic 5-body phase space sampling (sample_5body_phasespace()). $\phi$ and $\theta$ between 1 random pair of 5 -body state.

multi-particle reactions are used. On the contrary, the stochastic criterion can also be used without multi-particle reactions for all cases where the geometric criterion is applied. By default, the geometric collision criterion is used and therefore, multi-particle reactions are disabled.

In the following, examples are given, how multi-particle reactions are configured. The examples quote the relevant section of the configuration file (Collision_Term) that needs to be changed. For all examples, a small enough time step has to be set (Delta_Time: 0.1 is a good starting point, see Section 5.1.5 and the end of this section). A good general starting point for first calculations using multi-particle reaction are the example input files for the multi-particle box (under input/multi_particle_box in the SMASH source [209]) and the detailed balance tests for multi-particle reaction in the analysis suite [225].

The mesonic 3 -to- 1 reactions $\left(3 \pi \rightarrow \omega, 3 \pi \rightarrow \phi\right.$ and $\left.\eta \pi \pi \rightarrow \eta^{\prime}\right)$ are configured as follows:

Collision_Term:

Collision_Criterion: Stochastic

Multi_Particle_Reactions: ["Meson_3to1"]

Note that without including the inverse reaction i.e. the Dalitz decays in the decay modes file as shown below, no 3-to-1 reactions are performed since detailed balance would be broken.

$\omega$

$0.893 \quad 1 \quad \pi^{+} \pi^{-} \pi^{0}$

$\varphi$

$0.1521 \pi^{+} \pi^{-} \pi^{0}$

$\eta$

$0.426 \quad 0 \quad \pi^{+} \pi^{-} \eta$

$0.228 \quad 0 \quad \pi^{0} \quad \pi^{0} \quad \eta$ 
For the deuteron 3-to-2 reaction, the configuration involves excluding the 2-to- 2 reactions that involve the $d^{\prime}$ resonance, which is done by including all 2-to-2 reactions but the $d^{\prime}$ ones ("PiDeuteron_to_pidprime" and "NDeuteron_to_Ndprime"). The $\pi d \leftrightarrow N N$ as 2-to-2 d reaction without the $d^{\prime}$, is included on purpose.

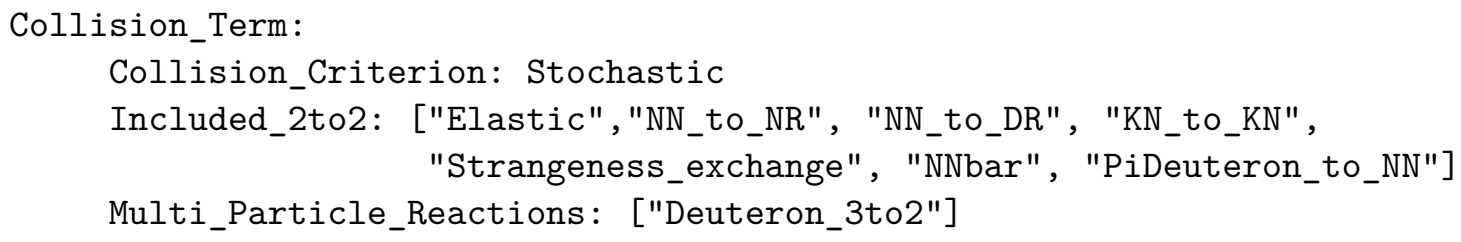

Note that when configuring the deuteron 3 -to-2 reaction, the respective 2 -to- 3 reaction is automatically included as well.

Furthermore, the particles file has to include the deuteron and $d^{\prime 6}$ as shown below.
d 1.87560
$+1000010020$
d' 1.88560 .1
- 1000010021

And, since the $d^{\prime}$ is unstable its decay mode also has to be included when running with 3 -to-2 reactions. The implied resonance formation of $d^{\prime}$ is ignored in this case.

$d^{\prime}$

1. $1 \quad \mathrm{~N}$ N

The $d^{\prime}$, even though not produced in the calculation, is necessary since the $d$ cross-section is parametrized using the $d^{\prime}$ pole mass and decay width as parameters. This is also the reason why the 2-to-2 reactions involving the $d^{\prime}$ need to be excluded. Otherwise, the deuteron 3 -to-2 reactions would be double-counted.

To employ the 5 -to- 2 reaction $5 \pi \leftrightarrow N \bar{N}$ in both directions, the following needs to be used.

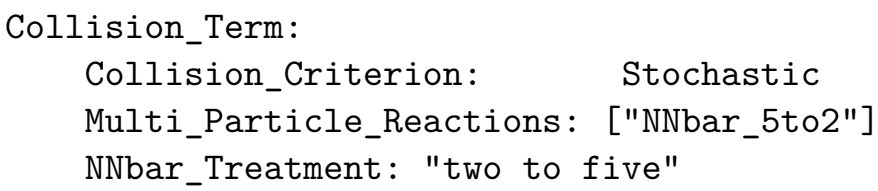

Future versions of SMASH might change resonance properties, such as branching fractions or pole masses. So, if important for a calculation, the numbers quotes above need to be verified before. Also, new 2-to-2 reactions might be added in the future, so it might

\footnotetext{
${ }^{6}$ While using the same parameterization of the $d$ cross-section ensures comparability to the 2-to-2 approach, the need to include the $d^{\prime}$ when not needing it as a degree of freedom in the calculation is not ideal. An improvement left for the future is to use a direct parametrization of the $d$ cross-section in the future, which would avoid the need of listing the $d^{\prime}$ in the particle and decay mode file.
} 
be necessary to ensure that the list of all 2-to-2 reactions but the $d^{\prime}$ ones is still up-todate. Of course, the different multi-particle reactions can be combined as necessary for a calculation.

A couple of common warnings and errors occur while running SMASH with the stochastic criterion and multi-particle reactions. The program errors and does not start if the config is not correct e.g. when trying to run a multi-particle reaction with the geometric criterion, otherwise detailed balance would be broken. The collision probability is not limited to 1 by definition. The user needs to ensure that this is the case by choosing appropriate parameters, especially a small enough timestep. Should the collision probability exceed 1, SMASH throws an error by default. While a probability larger than 1 is a conceptual issue, it is not affecting the calculated results if it is rare. Therefore, for production runs, the additional config option Only_Warn_For_High_Probability: True can be set. The user must ensure that this warning only occurs rarely e.g. for extreme kinematic cases or diverging cross-sections. As shown in Section 5.1.5, an important assumption for the stochastic criterion (for two- or multi-particle reactions) is that not more than 1 reaction per timestep per particle is accepted. Should more than one reaction be found in SMASH, all reactions after the first one are discarded. The number of discarded reactions needs to be low. Otherwise the mentioned assumption is invalid. In testing, the effects of too many discarded reactions are seen starting from $1 \%$ of discarded reaction for all performed reactions. Therefore, a warning is printed at the end of the calculation if this percentage is exceeded. The results are not affected necessarily. But, if this warning is printed, it needs to be cross-checked whether e.g. a smaller timestep changes the results.

\section{A.4 Performance of multi-particle reactions}

In the original publication [134], which discusses the stochastic criterion (called local ensemble method in the publication), its performance i.e. computing time advantages, are reported. For the current implementation of SMASH, the opposite is found: The stochastic criterion is slower than its geometric counterpart. The main reason for not finding a performance advantage is the number of pairings built for the particles inside each cell. In SMASH, all possible pairs are checked for collisions, which results in $N(N-1) / 2$ pairs and calculations of the collision probability for $N$ (test) particles within a cell . The authors of [134] only choose one random set of possible pairs, which are only $N / 2$ combinations. To account for the missed pairings, the collision probability $P$ is scaled up as

$$
P^{\prime}=P \frac{N_{\text {possible }}^{\text {pairs }}}{N_{\text {build }}^{\text {pairs }}}=P \frac{N(N-1) / 2}{N / 2} .
$$

Note that the first part of the equation also applies for multi-particle reactions. Even when not all particles can be part of an n-particle pairing, the number of possible nparticle pairings can still be used to scale the probability. The significant reduction in combinations improves the computing time, which would only scale linearly with $N$. Even though this would improve the runtime, the advantage in SMASH is not expected to be as significant as found in [134] due to the employed nearest neighbor search for the geometric criterion (see Section A.2.1), which already leads to a reduction from the full quadric scaling. The observed increase in runtime for the stochastic criterion compared to the geometric criterion results from the strict timestep-based approach, which requires small timesteps. 
The focus of this work is to obtain correct physical results and verifying the stochastic approach. For this purpose, it abstains from optimizing prematurely. In pairing all particles, also the same routine as for the geometric criterion is kept. This is valuable to compare both criteria, which is done extensively in the presented results to verify the approach. Therefore, the explained optimizations above and in the following are left for future work and can be done based on the rich verification presented throughout this thesis. The observed slowdown is further enhanced when including multi-particle reactions in the calculation, leading to even more possible pairings. In particular, for the introduced 5-body reactions, combinatorics are the largest issue. At a certain number of particles inside a cell, the whole calculation time is used for looping through all 5-particle pairings. Here, reducing the combinatorics by scaling the probability would help. For the 3-body reactions, the main bottleneck at the moment is the integration of the 3-body phase space, which is performed for every 3-particle pairing that is found. Similar to the 5-body phase space, a parametrization of the 3-body phase space could improve the performance. More general optimizations could prevent the creation and thereby memory allocation of action objects for all parings in SMASH. Memory is allocated regardless of the action being accepted or not, which leads to many memory operations being performed even for directly discarded pairings. To reduce the memory allocations for 5-body reactions, where a lot of pairings are possible, an action object is already only created if all particles are pions since the only possible 5-body reaction is $5 \pi \rightarrow N \bar{N}$ at the moment. Another idea is to parallelize the search for actions within each cell. Since for the stochastic criterion, collisions only happen within each grid cell, the collision search could directly be parallelized.

Two other possibilities related to optimizing the runtime are to implement adaptive timesteps and a dynamic switching between the geometric and stochastic criterion. Adaptive timesteps firstly could prevent probabilities larger than 1 by reducing the timestep automatically should such a probability be calculated (as done in BAMPS [111]). At the same time, if the system becomes more dilute, the timestep could also be increased without violating the assumption of only 1 interaction per timestep per particle. This idea is related to the dynamic switching of criteria. Since the stochastic criterion requires a higher number of particles inside each cell to properly function (cf. Section 5.1.5), the criterion could be switched for the late, dilute stages of a collision that are mainly free-streaming. In this stage, the geometric criterion is more applicable and significantly faster since it does not need any timesteps and propagates from interaction to interaction. The main challenge for both of these optimizations is to find robust a priori criterion when either the adaptation of the timestep or the switching of criteria should occur. For example, it might be natural to consider a density basis for the switching of the criteria. However, considering the example of a heavy-ion collision, the density varies widely over time and over phase-space regions. The spectators fly unperturbed with their ground state density away, in the collision zone, the density is high and between spectators and the collision zone, the density is mostly zero. Therefore, finding a good density criterion that takes all this into account and works for different beam energies is complex. 


\section{Appendix B}

\section{More differential dilepton predictions for AuAu collisions}

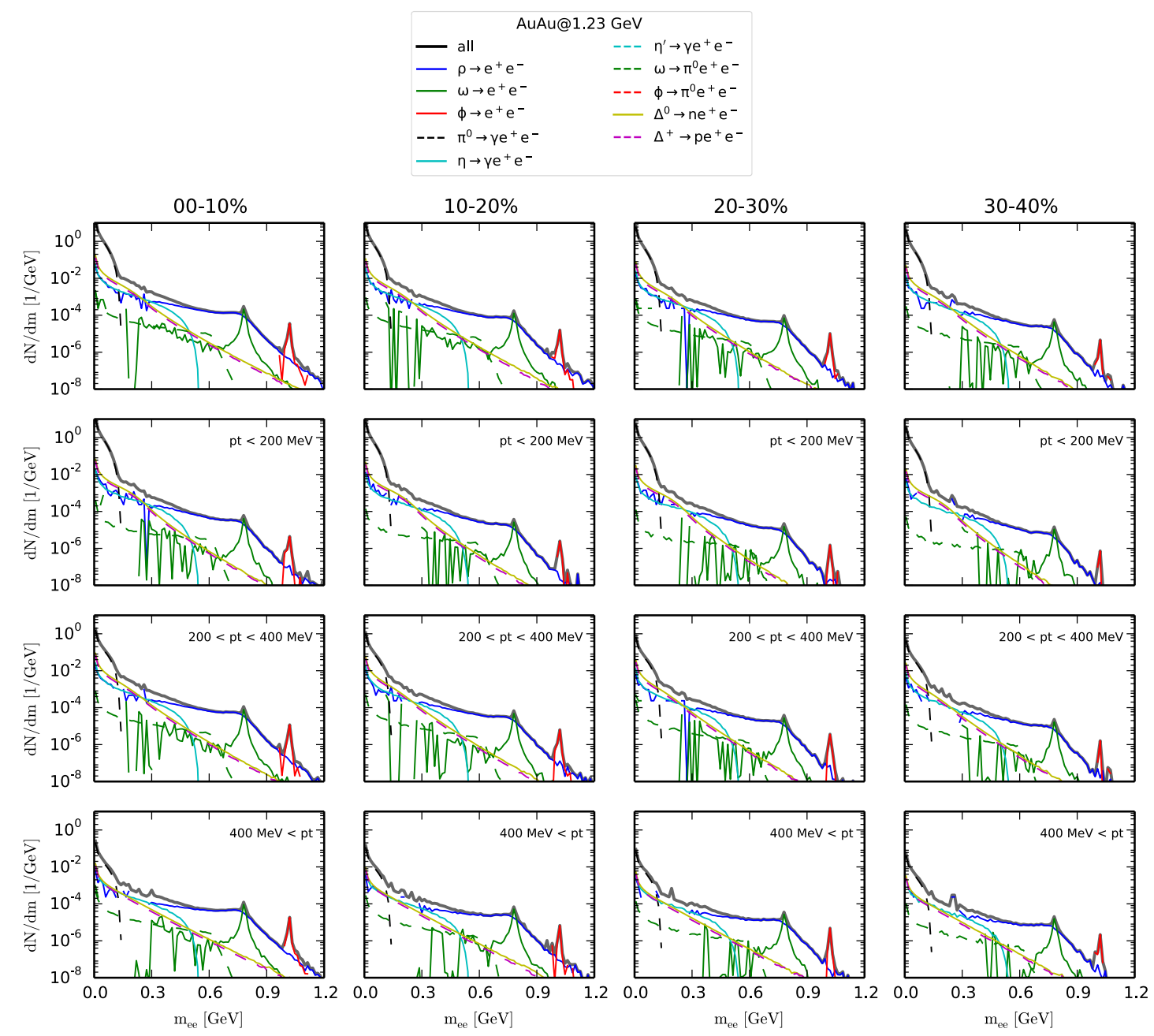

Figure B.1: Invariant mass spectra of dielectrons produced by AuAu collisions at $E_{\mathrm{Kin}}=$ $1.23 A \mathrm{GeV}$ for different centrality classes (columns) and $p_{T}$ windows (no $p_{T}$ cut for top row). 
To complement the over centrality classes and momentum integrated invariant mass spectrum of the dielectrons production in $\mathrm{AuAu}$ collisions at $E_{\mathrm{Kin}}=1.23 \mathrm{~A} \mathrm{GeV}$ in Figure 3.18 from Section 3.1.2, more differential spectra are presented in this appendix in Figure B.1, B.2 and B.3. The total, $\rho$ and $\omega$ contributions are the same as displayed in Figures 3.25, 3.26 and 3.27. The presented spectra match the upcoming experimental analysis and provide predictions over the whole range of phase space. In particular, probing multiple invariant mass windows appears to be promising. As seen in Figure B.2 and B.3, different invariant mass cuts cause different contributions to be dominant and allow to study especially the vector meson decays. Linestyles for all figures are given by the legend in Figures 3.25.
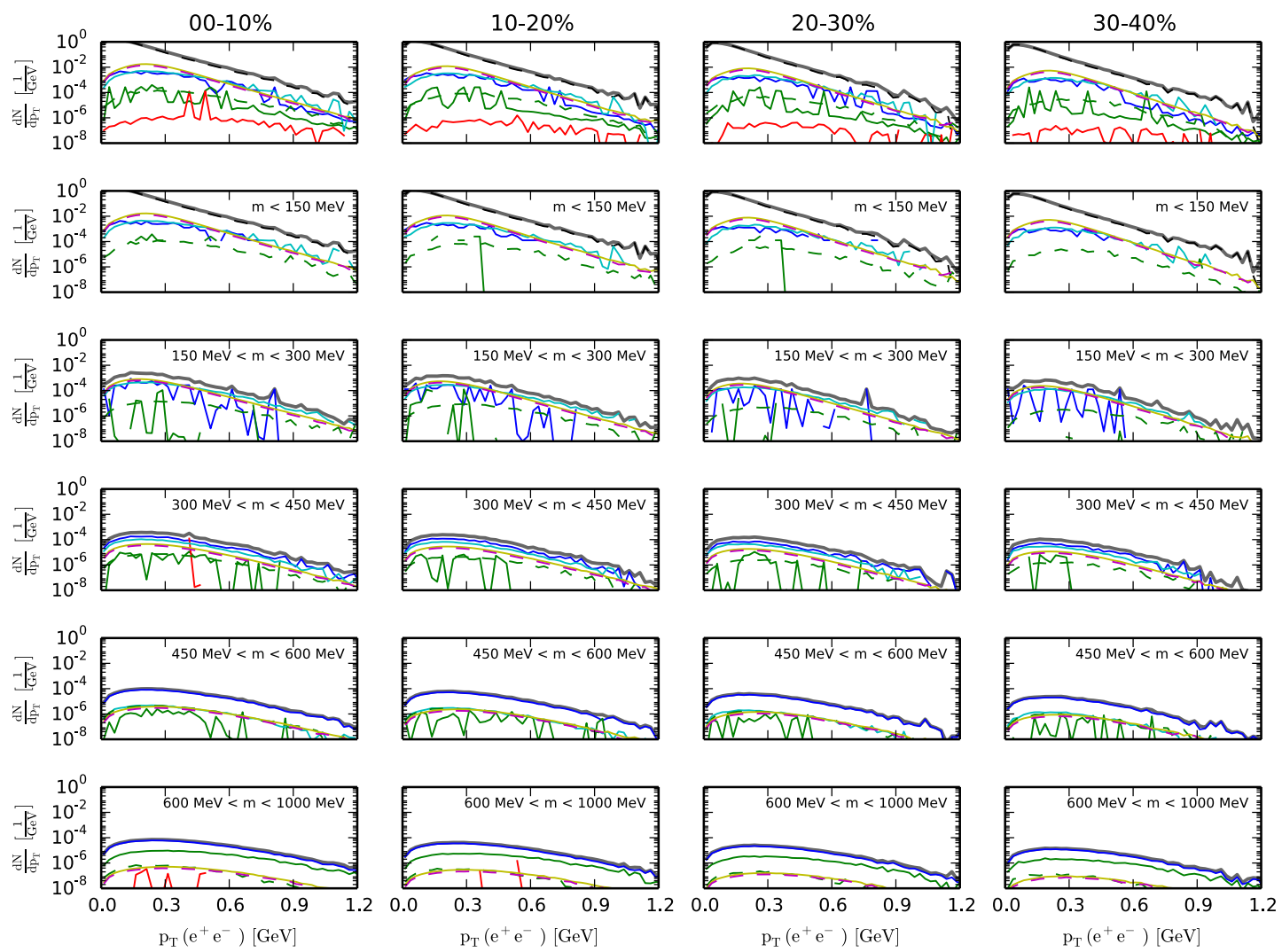

Figure B.2: Transverse momentum spectra of dielectrons produced by AuAu collisions at $E_{\text {Kin }}=1.23 \mathrm{~A} \mathrm{GeV}$ for different centrality classes (columns) and invariant mass windows (no invariant mass cut for top row). Linestyles as in B.1. 

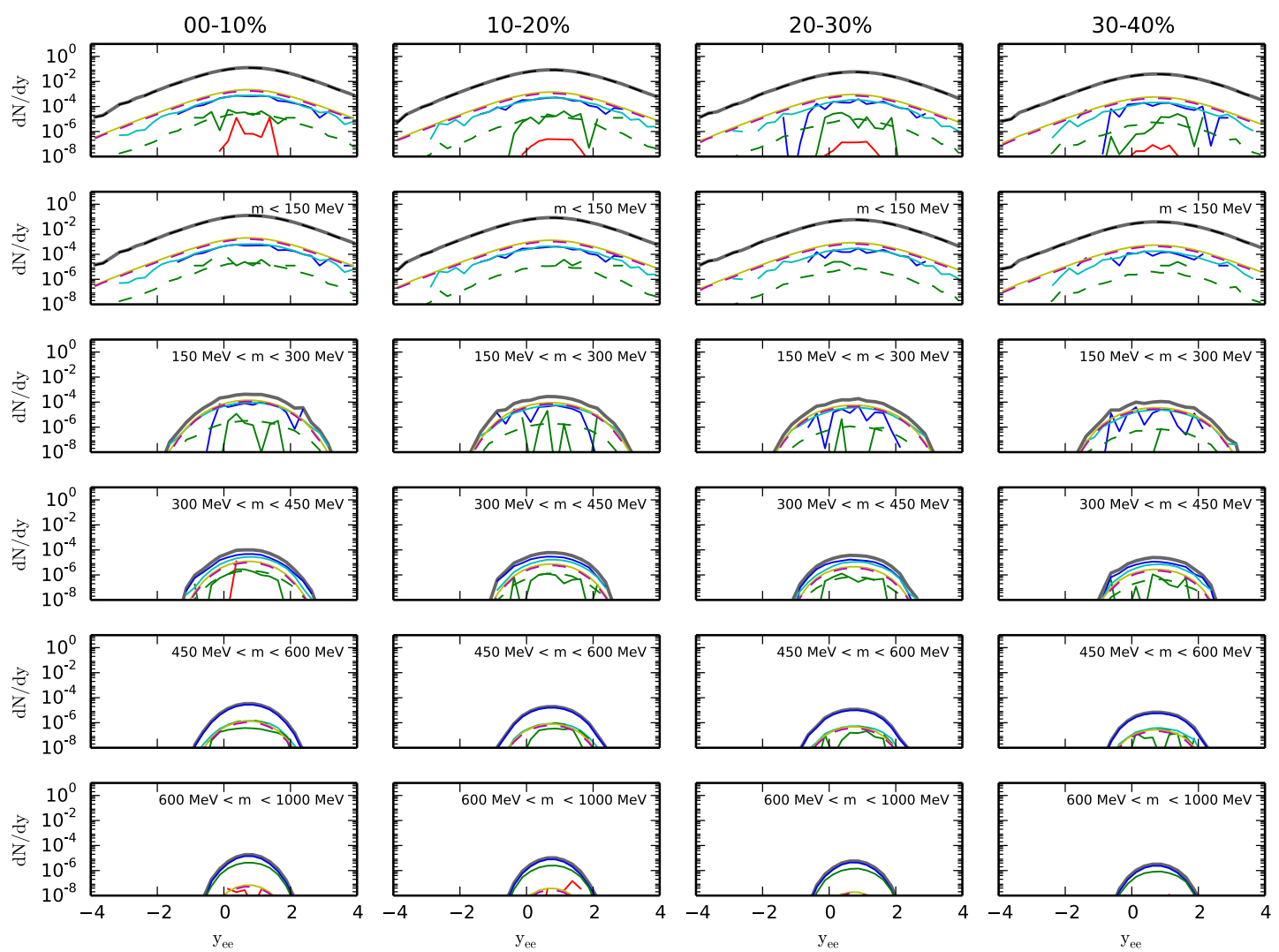

Figure B.3: Rapidity spectra of dielectrons produced by $\mathrm{AuAu}$ collisions at $E_{\mathrm{Kin}}=$ $1.23 A \mathrm{GeV}$ for different centrality classes (columns) and invariant mass windows (no invariant mass cut for top row). Linestyles as in B.1. 
